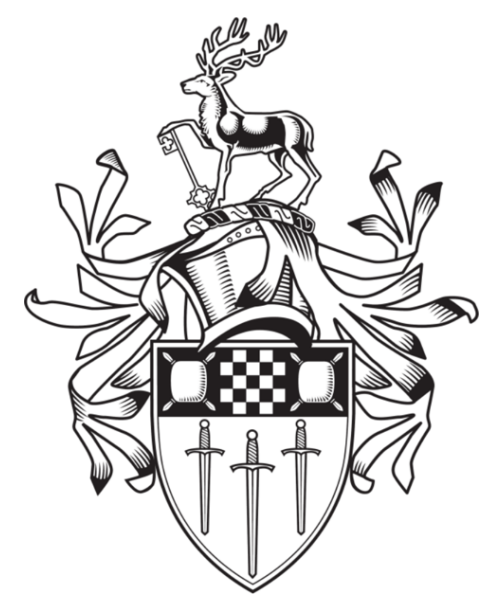

\title{
A Multicellular Architecture towards Low-Cost Satellite Reliability
}

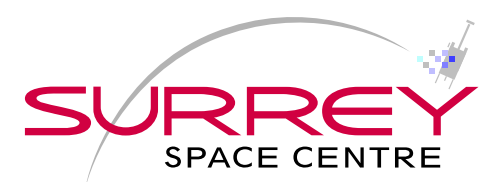

Alexander Olaf Erlank

Supervisor: Dr. C. P. Bridges

Surrey Space Centre

University of Surrey

Thesis Submitted in Fulfilment of the Requirement for the Degree of Doctor of Philosophy January 2018 



\section{Statement of Originality}

This thesis and the work to which it refers are the results of my own efforts. Any ideas, data, images, or text resulting from the work of others (whether published or unpublished) are fully identified as such within the work and attributed to their originator in the text, bibliography, or in footnotes. This thesis has not been submitted in whole or in part for any other academic degree or professional qualification. I agree that the University has the right to submit my work to the plagiarism detection service TurnitinUK for originality checks. Whether or not drafts have been so assessed, the University reserves the right to require an electronic version of the final document (as submitted) for assessment as above.

Alexander Olaf Erlank

January 2018 



\section{Acknowledgements}

Approximately four years ago, nearing the end of my studies at Stellenbosch University in South Africa, I was preparing to present at a small satellite conference in Belgium. I had become fascinated by space exploration and was determined to find my way into the space industry.

Despite my aims of networking while at the conference, I did not leave with a job. However, through the efforts of my Master's supervisor, Professor Steyn, I had been presented with an entirely unexpected opportunity: a PhD position at Surrey University. I packed my bags and, with my professor's encouragement and parents' blessing, moved to England.

It turns out a $\mathrm{PhD}$ is hard work and requires many hours behind a computer writing MATLAB scripts and in a lab soldering and debugging tiny circuits. However, this thesis is just one result of my time at Surrey. I have made (hopefully) lifelong friends from all over the world, travelled more than I could ever have hoped for, and fulfilled a dream of working on real satellite hardware. Thus, as I finally reach my word count, it is time to thank everyone who has made this experience possible.

Firstly, thank you to all the staff of the Surrey Space Centre for making it the world-class research facility that it is. Thank you to the support staff for always being friendly, helpful and efficient when dealing with my technical and administrative issues, and to the academics, who gave me the opportunities to work on fascinating side projects, including a high altitude balloon and the SMESAT CubeSat.

Next, I would like to thank my supervisor, Dr Christopher Bridges. Six months into my PhD, and still lacking a solid research topic, Chris academically adopted me after the unexpected departure of my first supervisor. With his guidance and abundance of enthusiasm, Chris quickly got my $\mathrm{PhD}$ on track. Over the following years, he continued to provide invaluable guidance on how to be a scientist (as opposed to an engineer) and never failed to entertain with inspiring stories. Able to advise equally well on tricky technical issues and philosophical research problems, Chris has been a mentor and friend. Thank you, Chris! 
I came to England expecting to pick up a British accent, but instead found myself surrounded by half of Europe (and some from further afield). While work on this thesis taught me about cell differentiation, reliability theory and Agent Computing, the friends I've made while at Surrey taught me about truffles, Polish vodka, arancini, and bacalhau. They have been office mates, house mates, team mates and travel companions. Thank you for joining me on Fridays in Wates for a beer, organising weekend activities and nights out, dressing up and attending our house parties, inviting me to your countries and for visiting mine!

Finally, to my family: Olaf, Daleen and Marcus. Thank you for giving me the opportunity to live and study in Stellenbosch, where my love for engineering, space and travel flourished. Thank you for encouraging me to follow my passion and make the most of unexpected opportunities, even if it means moving half-way around the world. And finally, thank you for reminding me that South Africa will always be home. 


\begin{abstract}
While small, low-cost satellites continue to increase in capability and popularity, their reliability remains a problem. Traditional techniques for increasing system reliability are well known to satellite developers. They include the use of radiation-hardened and screened components, extensive cold redundancy and thorough test campaigns. However, the implementation of these techniques on small, low-cost satellites is often limited due to intrinsic mass, volume and budgetary restrictions. Aiming for graceful degradation, therefore, may be a more promising route.

Inspired by the robustness of single-celled and multi-cellular biological organisms, bioinspired computing systems, multi-agent systems, and modular spacecraft concepts, this work presents the design, implementation and analysis of an artificial, cell-based system architecture. Named the Satellite Stem Cell Architecture, the proposed system aims to replace a significant portion of a typical satellite's bus avionics with a set of initially identical, mass produced, artificial cells. Analogous to their biological counterparts, the artificial cells can differentiate during runtime to take on a variety of tasks, thanks to a set of artificial proteins. Each cell reconfigures its own proteins within the context of a system-wide, distributed task management strategy. In this way, essential tasks can be maintained, even as system cells fail.

The Satellite Stem Cell Architecture differs from existing bio-inspired computing systems by extending the concept to include reconfigurable interfaces to real-world sensors and actuators, and by its inclusion of a set of middleware which turns each cell into a multiagent platform. Furthermore, an emphasis is placed on practical applicability, with power consumption, volume and production cost driving the implementation. A detailed description of the artificial cell hardware, and multi-agent middleware, is given. Additionally, two CubeSat-scale, practical implementations of the architecture are described. While one, which forms the payload interface computer of the SMESAT CubeSat, demonstrates only a subset of the proposed multicellular features, the other is a full testbed based on two artificial cells of four proteins each.
\end{abstract}


To compare the reliability of the proposed architecture to traditional forms of redundancy, an analytical reliability equation is derived for predicting the lifetimes of multicellular systems. It is shown that determining the optimal configuration of proteins per cell and cells per system is complex, as different configurations are optimal during different phases of the mission lifetime. Nevertheless, a set of trends in system behaviour are discovered, which will prove useful to system designers. Using a purpose-developed, multicellular simulation environment, the results of the analytical work are verified, and further problems relating to peripheral interfaces and cross-strapping are investigated.

Finally, using measured characteristics of the implemented testbed and the derived analytical lifetime predictions, the Artificial Stem Cell Architecture is compared against traditional CubeSat and microsatellite avionics suites. The results show that the proposed architecture gives increasing reliability and performance benefits with increased scale, and that while its power consumption overheads make it prohibitive for implementation on CubeSats, it is well-suited to microsatellites. 


\section{Table of Contents}

List of Figures $\quad$ xiii

$\begin{array}{ll}\text { List of Tables } & \text { xix }\end{array}$

List of Acronyms and Abbreviations $\quad$ xxi

1 Introduction 1

1.1 Research Motivation . . . . . . . . . . . . . . . . . 2

1.2 Aims \& Objectives . . . . . . . . . . . . . . . . 4

1.3 Contributions ....................... 5

1.4 Thesis Structure . . . . . . . . . . . . . . . . . . . 6

2 Background 9

2.1 Rise of the Small, Low-Cost Satellites . . . . . . . . . . . . . . . . . . 9

2.1 .1 Important Examples . . . . . . . . . . . . . . . . . 11

2.1.2 The Future of Small, Low-Cost Satellites . . . . . . . . . . . . . 13

2.2 Reliability Overview . . . . . . . . . . . . . . . . . . . 15

2.2.1 Fault Avoidance and Fault Tolerance . . . . . . . . . . . . . . 16

2.2.2 Hardware Redundancy . . . . . . . . . . . . . . . . . . . . 18

2.3 Satellite Reliability . . . . . . . . . . . . . . . . . . . . . . . 19

2.3.1 The Poor Reliability of Low-Cost Satellites . . . . . . . . . . . . . 21

2.3.2 CubeSat Survey - Finding a Recipe for Success . . . . . . . . . . 26

2.4 Summary . . . . . . . . . . . . . . . . . . . . 32

3 Sources of Inspiration $\quad 35$

3.1 Bio-Inspiration . . . . . . . . . . . . . . . . . 35

3.1 .1 Unicellular Life . . . . . . . . . . . . . . . . 36

3.1 .2 Multicellular Life . . . . . . . . . . . . . . . . . . 38 
3.1.2.1 Cells versus Subsystems . . . . . . . . . . . . . . 39

3.1 .3 Natural Redundancy . . . . . . . . . . . . . . . . . . . . 40

3.1.3.1 A Closer Look at Differentiation . . . . . . . . . . . . 41

3.1.3.2 Decentralised Architecture . . . . . . . . . . 42

3.1.3.3 Programmed Cell Death . . . . . . . . . . . . . . 44

3.1.4 A Multicellular Satellite . . . . . . . . . . . . . . . . . . . 45

3.2 Bridging Research Fields . . . . . . . . . . . . . . . . . . . . 47

3.2.1 Bio-Inspired Processors _ . . . . . . . . . . . . . . . 47

3.2.1.1 Embryonics . . . . . . . . . . . . . . . 47

3.2 .1 .2 POEtic . . . . . . . . . . . . . . 49

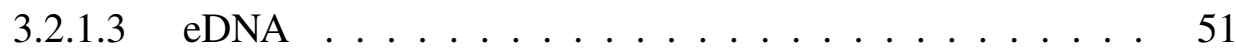

3.2 .2 Multi-Agent Systems . . . . . . . . . . . . . . . . 53

3.2.2.1 Traditional Agent Platforms . . . . . . . . . . . . 54

3.2.2.2 Embedded Agent Platforms . . . . . . . . . . . . . 54

3.2.2.3 Agents in Space . . . . . . . . . . . . . . . . . . . . . . . . . . . . . 55

3.2.3 Modular Satellite Concepts . . . . . . . . . . . . . . . . . . . . 56

3.2.3.1 Space Plug-and-Play . . . . . . . . . . . . 56

3.2.3.2 DARPA Phoenix Project . . . . . . . . . . . 58

3.2.4 Discussion and Expected Novelties . . . . . . . . . . . . . . 60

3.3 Summary . . . . . . . . . . . . . . . . . . 62

4 Artificial Stem Cells

4.1 Artificial vs Biological Cells . . . . . . . . . . . . . . . . 66

4.2 Artificial Cell Membranes and Peripheral Interfacing . . . . . . . . . . . 68

4.3 A Multicellular System . . . . . . . . . . . . . . . . . . . . . . 73

4.3.1 Inter-Protein and Inter-Cell Communication . . . . . . . . . . . 74

4.3.2 Artificial Mitosis . . . . . . . . . . . . . . . . . . . . 74

4.3 .3 Cell Death . . . . . . . . . . . . . . . . 75

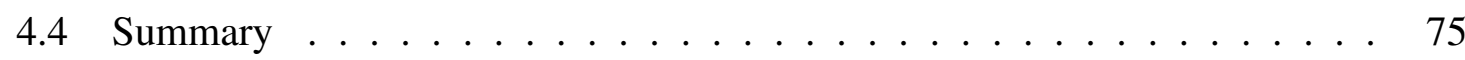

5 Hybrid Agent Real-Time Platform $\quad 77$

5.1 FIPA Abstract Architecture . . . . . . . . . . . . . . . 78

5.1.1 Agent Execution Environment . . . . . . . . . . . . . . 79

5.1 .2 Agent Management System _ . . . . . . . . . . . . . . . . 79

5.1.3 Agent Communication Channel . . . . . . . . . . . . 80

5.1.3.1 Physical and Data Link Layers _. . . . . . . . . 82

5.1.3.2 Network and Transport Layers . . . . . . . . . . . 82 
5.1.3.3 FIPA Higher-Level Protocols . . . . . . . . . . . . 84

5.2 Extended Features . . . . . . . . . . . . . . . . . . . . 86

5.2.1 Real-Time Messaging _. . . . . . . . . . . . . . . 86

5.2 .2 Task Allocation Entity . . . . . . . . . . . . . . . . . . . . . . . . . . . 87

5.2 .3 Redundancy . . . . . . . . . . . . . . . . . . . . 89

5.2 .4 Agency Services Failure . . . . . . . . . . . . . . . . . . . 89

5.3 Summary . . . . . . . . . . . . . . . . . . . . . 90

6 Reliability Analysis and Simulation $\quad 93$

6.1 Introduction to Analytical Reliability Analysis . . . . . . . . . . . . . . . . 94

6.2 Reliability Equation Derivation . . . . . . . . . . . . . . . . . . . 97

6.2.1 Common System Architectures ～. . . . . . . . . . . . . . . . 97

6.2.2 Multicellular Architectures . . . . . . . . . . . . . . . . . . 100

6.2.3 Understanding the System State PDF . . . . . . . . . . . . . . 105

6.3 System Reliability Sensitivity Analysis . . . . . . . . . . . . . . . . . 108

6.4 Verification through Simulation . . . . . . . . . . . . . . . . 115

6.5 Effect of Peripherals . . . . . . . . . . . . . . . . . . . . 117

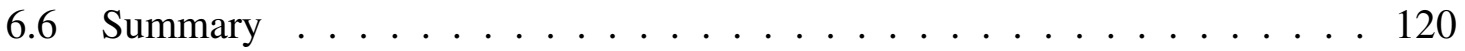

7 Multicellular System Implementations $\quad 123$

$7.1 \quad$ SMESAT . . . . . . . . . . . . . . . . . . . . . . . 124

7.1.1 Initial System Design . . . . . . . . . . . . . . . . . . 125

7.1.2 Cellularised Design . . . . . . . . . . . . . . . . 126

7.2 CubeSat-Scale Multicellular System Testbed . . . . . . . . . . . . . . . . 128

7.2.1 GPIO Circuitry Prototyping . . . . . . . . . . . . . . . 130

7.2.2 Artificial Cell Implementation . . . . . . . . . . . . . . . . 136

7.2.3 HARP Middleware Implementation . . . . . . . . . . . . . . 140

7.2.4 Demonstrating Functionality . . . . . . . . . . . . . . . . . . . 142

7.2.4.1 Task Allocation Demonstration . . . . . . . . . . . . 143

7.2.4.2 Continuity of Runtime Variables Demonstration . . . . . 144

7.2.4.3 Cross-Strapping Demonstration . . . . . . . . . . . . 145

7.2.5 Performance and Scalability Benchmarking . . . . . . . . . . 146

7.2.5.1 Processing Capacity and Power Consumption . . . . . 146

7.2.5.2 Housekeeping Bus Overheads . . . . . . . . . . . . . . . 148

7.2.5.3 AMS Performance and Router Bandwidth . . . . . . . . 149

7.2.5.4 GPIO Cross-Strapping Limitations . . . . . . . . . . 150

7.3 Multicellular Satellite Case Studies . . . . . . . . . . . . . . . . . . . . 152 
7.3.1 CubeSat ADCS . . . . . . . . . . . . . . . 152

7.3.1.1 Reliability Comparison . . . . . . . . . . 156

7.3.1.2 Performance and Overheads . . . . . . . . . . . . 158

7.3.2 MicroSat Avionics . . . . . . . . . . . . . . . . . . . . 159

7.3.2.1 Reliability Comparison . . . . . . . . . . . 162

7.3.2.2 Performance and Overheads . . . . . . . . . . . 162

7.3 .3 Case Studies Conclusion . . . . . . . . . . . . . . . . . . . . 164

7.4 Summary . . . . . . . . . . . . . . . . . 165

8 Conclusions $\quad 167$

8.1 Publications . . . . . . . . . . . . . . . . . . 171

8.2 Future Work . . . . . . . . . . . . . . . . . . . . 172

8.2.1 Downtime Prediction . . . . . . . . . . . . . . . . . . 172

8.2.2 Specialised Task Allocation Strategies . . . . . . . . . . . . . . . 172

8.2.3 Semantic Language . . . . . . . . . . . . . . . . . . . 173

8.2.4 Agent-Based Ground Station and Communication Gateways . . . . 173

8.2.5 Next Generation Artificial Cells . . . . . . . . . . . . . . . . . . 173

$\begin{array}{ll}\text { References } & 175\end{array}$

Appendix A Parametric CubeSat Database 185

$\begin{array}{lll}\text { Appendix B } & \text { Artificial Cell Schematics } & 191\end{array}$ 


\section{List of Figures}

2.1 NASA's $2623 \mathrm{~kg}$ Landsat 8 satellite and ESA's $2300 \mathrm{~kg}$ Sentinel satellite . . 10

2.2 UoSat-1, the first microsatellite with a reprogrammable onboard computer 1981, and a number of PlanetLab CubeSats awaiting launch 2014 . . . . . . 12

2.3 The AAReST mission aims to demonstrate the assembly of a large mirror composed of small satellites on orbit cite. . . . . . . . . . . . . . 14

2.4 SpaceWorks' prediction of the number of sub-50 kg satellites to be launched over the next few years. . . . . . . . . . . . . . . . . 15

2.5 Traditional satellite reliability based on empirical failure data of 1548 satellites (a) and the most common causes of failure within the first year (b). . . 20

2.6 Empirical reliability plots for three mass classes of satellites. . . . . . . . . 21

2.7 Empirical reliability plots of different classes of small satellites, based on Guo, Monas and Gill's survey of 222 satellites launched between 1990 and

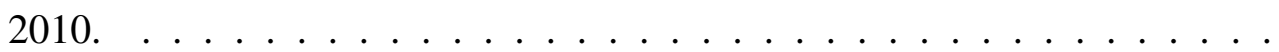

2.8 Mission success rates of a group of 94 nano satellites $(<10 \mathrm{~kg})$ launched between 1957 and 2009. . . . . . . . . . . . . . . . . .

2.9 Mission success rates of the first 100 CubeSats, and the primary causes of failure . . . . . . . . . . . . . . . . . . 24

2.10 CubeSat mission success rates versus number of redundant core subsystems (a), and number of COTS core subsystems (b) . . . . . . . . . . . . .

2.11 CubeSat mission success rates versus ADCS complexity (a), and system architecture type [0-star, 0-single-master bus, 0-multi-master bus] (b). . . .

2.12 CubeSat mission success rates versus development time. The last category (6) includes missions which took longer than six years to develop. . . . . . 31

3.1 Extremophiles in geysers and at the bottom of the ocean. . . . . . . . . 37

3.2 Zebrafish and Newts. . . . . . . . . . . . . . . . . . 41

3.3 The three-level hierarchy of the Embryonics architecture. . . . . . . . . . . 48 
3.4 The organic subsystem of the POEtic architecture . . . . . . . . . . . . . 50

3.5 The eDNA architecture is composed of a grid of eCells and a mesh communication network. . . . . . . . . . . . . . . . 52

3.6 The Space Plug-and Play architecture. . . . . . . . . . . . . . . 56

3.7 The SPA network architecture. . . . . . . . . . . . . . . . . 57

3.8 Comparison between traditional satellites of varying sizes, and their 'satletised' counterparts. . . . . . . . . . . . . . . . . . 59

3.9 Different scales of system cellularisation based on cells of increasing functional scope. . . . . . . . . . . . . . . . . . . 60

4.1 A simplified depiction of the differentiation process in a biological cell, and the proposed artificial implementation. . . . . . . . . . . . . 66

4.2 Block diagram of the generic I/O circuitry which allows proteins to interface directly to a variety of external peripherals. . . . . . . . . . . . .

4.3 The first peripheral interfacing scheme gives each cell a set of communal I/O channels, which can be accessed by multiple proteins on the same cell through multiplexers. . . . . . . . . . . . . . . . 70

4.4 The second peripheral interfacing scheme gives each protein its own I/O channels, which can be switched onto different peripherals using multiplexers. 70

4.5 The third peripheral interfacing scheme removes multiplexers altogether. Instead, I/O signal conflicts are avoided through a high-impedance mode I/O mode and through inter-protein cooperation (in software) . . . . . . . . . 70

4.6 The detailed design of two artificial cells within a multicellular system. Any protein on a cell can take on the role of macro-molecular machinery. Peripherals can be cross-strapped to an arbitrary number of proteins, as determined by the peripheral's criticality. In addition, peripherals can be cross-strapped between cells. . . . . . . . . . . . . . . . . . . .

5.1 Schematic representation of an agency based on the FIPA Abstract Archtecture (a), and an agent platform based on the HARP middleware running on an artificial stem cell (b). Note that the Directory Facilitator is an optional component which is not currently implemented in the HARP middleware. . 78

5.2 The relationship between the OSI network stack and the FIPA communication stack. . . . . . . . . . . . . . . . . .

5.3 The HARP middleware communication stack, supporting both ACL and real-time messaging. Layers shown in grey implement FIPA protocols. . . . 
5.4 An example HARP ACL message (a), and a schematic representation of the Query FIPA interaction protocol.

5.5 Flowchart depicting the task allocation strategy executed concurrently and asyncronously by every cell's Task Allocation Entity. . . . . . . . . . . .

6.1 Failure rate, probability density and reliability plots of a system known to suffer from continuous random failures. . . . . . . . . . . . . . . 95

6.2 The effect of the Weibull Distribution scale parameter on failure rate, probability density and reliability plots . . . . . . . . . . . . . . 96

6.3 The bathtub reliability curve . . . . . . . . . . . . . . . . 96

6.4 Schematic representation of a single-string system architecture. . . . . . . . 99

6.5 Schematic representation of a multi-string system architecture. . . . . . . . 99

6.6 Schematic representation of a cross-strapped, multi-string system architecture. 99

6.7 Schematic representation of an n-choose-k system architecture. . . . . . . 99

6.8 Schematic representation of a simple multicellular system composed of two cells of two proteins each. . . . . . . . . . . . . . . . . . . . . 101

6.9 An example of a cell's discrete state probability density function, which reflects the probability of a specific number of proteins being functional at a given time.

6.10 Comparison of the reliability functions of different 24-task multicellular system configurations.

6.11 Comparison of the hazard functions of different 24-task multicellular system confgurations. . . . . . . . . . . . . . . . . . . . 104

6.12 The system state PDF changes over time. It is shown here as continuous for clarity, but is actually discrete. Able proteins are those which are functional and not reserved for MM duty. . . . . . . . . . . . . . . . . . . . 106

6.13 The effect of number of cells on the system state PDF. Excluding the peak at zero, the number of peaks is equal to the number of cells in the configuration. 106

6.14 The effect of proteins per cell on the system state PDF. As the number of proteins per cell increases, the peaks move further apart and become more defined. . . . . . . . . . . . . . . . . . . . . . 107

6.15 The effect of common component reliability on the system state PDF. Common component reliability affects the height of the peaks, but not their location. As common component reliability increases the distribution is shifted to the later peaks. . . . . . . . . . . . . . . . . . . . . 107 
6.16 Comparison of the sensitivity of various system architectures to total number of tasks. Each system has 6 tasks and a level of redundancy equal to triplestring. In traditional architectures, tasks are performed by subsystems, while in a multicellular architecture they are performed by proteins. At any point in time a multicellular architecture is the most reliable, however, which configuration is optimal changes during the system's lifetime. . . . . . . . . 109

6.17 Similar to Figure 6.16, but for systems featuring 12 (a) and 24 (b) tasks respectively. The reliability benefit of multicellular architectures is seen to increase with the total number of system tasks. . . . . . . . . . . . . 109

6.18 Comparison of the sensitivity of various system architectures to loading. Loading is defined as the number of active subsystems or proteins compared to the total number of subsystems/proteins. Each system has 12 tasks to run. At high loading, a single-celled architecture performs best. . . . . . . . . . 111

6.19 Similar to Figure 6.18, but for systems that are 33\% (a) and $25 \%$ (b) loaded. The lower loading is achieved by adding additional strings or proteins. Crossstrapped and multicellular architectures respond similarly to changes in loading. Amongst multicellular configurations, lower loading favours configurations featuring multiple, smaller cells. . . . . . . . . . . .

6.20 Comparison of the sensitivity of various architectures to common component reliability. Here, system reliability is measured with a single metric, MTTF, instead of a whole reliability plot. Multicellular architectures, and especially those featuring few cells, are seen to benefit more from increases in common component reliability than traditional architectures. . . . . . . . . . . 113

6.21 Reliability plots of systems with common component reliabilities from the low (a) and high (b) ends of Figure 6.20's scale. At low common component reliabilities (a), multicellular architectures perform comparably to crossstrapped designs. However, at high common component reliabilities (b), multicellular architectures have a clear reliability advantage. . . . . . . . 113

6.22 Comparison of the sensitivity of various system architectures to support component reliability. Multicellular architectures and cross-strapped architectures benefit similarly from initial increases in support component reliability. Thereafter, compared to cross-strapped architectures, multicellular systems experience more gradual diminishing returns. . . . . . . . . . . . . . . . 114 
6.23 The main window of the MATLAB multicellular system simulator. At the top of the window are controls for setting various parameters of the simulated system, as well as controls for stepping through failures and system reconfiguration. The bottom of the window shows a graphical representation of the current system state. In this case, a system composed of four cells of four proteins each is being simulated. . . . . . . . . . . . 116

6.24 The effect of increasing peripheral ratios on three different multicellular systems, composed of 12 (a), 24 (b), and 36 (c) proteins respectively. From all three plots it is clear that increasing peripheral ratios has a detrimental effect on system reliability. However, systems with peripheral ratios below $1 / 6$ can be reasonably approximated by an ideal system. . . . . . . . . . . .

7.1 A computer model of the SMESAT CubeSat being developed by the Surrey Space Centre. SMESAT's primary goal is to space-rate payloads from various small and medium enterprises across europe and Canada. . . . . . . . . . . 124

7.2 Schematic of the cellularised payload interface computer concept. . . . . . 127

7.3 Flight version of the cellularised PIC, mounted on top of the nano-CMG array. Note the four identical protein PCBs. . . . . . . . . . . . . . . 127

7.4 Clockwise from top left: All of the components of the PIC and CMG array, soft-stacked SMESAT, assembly montage. . . . . . . . . . . . . . . . 129

7.5 Prototype generic I/O circuitry. . . . . . . . . . . . . . . . . . 130

7.6 A section of the generic I/O circuitry, showing the half-bridge output stage and instrumentation amplifier input stage. . . . . . . . . . . . . 132

7.7 Demonstration of a pair of prototype generic I/O channels driving a brushedDC motor in full h-bridge configuration. . . . . . . . . . . . . . 132

7.8 Demonstration of a pair of prototype generic I/O channels completing a 100 $\mathrm{kb} / \mathrm{s} \mathrm{I} 2 \mathrm{C}$ transaction. . . . . . . . . . . . . . . . . . . . . 133

7.9 Fault tree analysis of the generic I/O circuitry. Only the TVS diode presents a potential single point of failure, provided a peripheral is cross-strapped. . 135

7.10 Implemented cell architecture based on four proteins interconnected by three buses. . . . . . . . . . . . . . . . . . . 137

7.11 Implemented artificial cell PCB layout. Notice the repetition of the generic I/O circuitry, and the symmetry of the four proteins. . . . . . . . . . . 139

7.12 Completed testbed cell (barring a few I/O channels), with important sections highlighted. . . . . . . . . . . . . . . . . . . . 139 
7.13 The complete multicellular testbed, composed of two cells. The toggle switches allow individual proteins to be switched off permanantly or momentarily, simulating failures. Both the inter-protein and inter-cellular bus traffic can be monitored through a terminal. . . . . . . . . . . . . . . . 142

7.14 Power consumption breakdown of a prototype artificial cell from the multicellular testbed. . . . . . . . . . . . . . . . . . . . . 147

7.15 Inter-protein CAN bus bandwidth utilisation by routine MM health checks and reprogramming procedures. . . . . . . . . . . . . . 148

$7.16100 \mathrm{~kb} / \mathrm{s}$ I2C clock waveform without cross-strapping (a), zoomed-in (b). . 151

$7.17100 \mathrm{~kb} / \mathrm{s} \mathrm{I} 2 \mathrm{C}$ clock waveform with cross-strapping between two I/O channels (a), zoomed-in (b). Note the increased rise time. . . . . . . . . . . . 151

7.18 The CubeADCS. . . . . . . . . . . . . . . . . . . 152

7.19 The architecture of the full-featured CubeADCS. . . . . . . . . . . 153

7.20 The architecture of a 'cellularised' CubeADCS, composed of two testbed cells and cross-strapped peripherals. . . . . . . . . . . . . . . . 154

7.21 Relaibility block diagrams of the CubeADCS operating in momentum-biased mode (a), magetic control/detumbling mode (b), and the equivalent cell-based design (c). C - common, S - support components. . . . . . . . . . . . 155

7.22 Comparison between the predicted reliabilities of the CubeADCS (operating in various modes) and the 2-cell (a) and 3-cell (b), theoretical, multicellular ADCSs. . . . . . . . . . . . . . . . . . . 157

7.23 SSTL's 100kg AlSat-1B microsatellite during the assembly phase. . . . . . 161

7.24 A screenshot of the MATLAB-based multicellular system simulator, configured to simulate a 10-cell system. . . . . . . . . . . . . . . 163

7.25 Comparison between the predicted reliability of a traditional cross-strapped microsatellite avionics suite, and a comparable 10-cell multicellular system. 163 


\section{List of Tables}

3.1 Number of specialised cell types versus total cell count in several organisms 39

4.1 The interfaces of typical satellite sensors and actuators. . . . . . . . . . 68

6.1 Simulated System Failure Models ～. . . . . . . . . . . . . . . 117

6.2 Comparison between Analytical and Simulated Reliability Functions . . . . 117

7.1 SMESAT Payload Interface Computer required interfaces . . . . . . . . . 126

7.2 The priorities, responsibilities and stack sizes of the FreeRTOS threads composing the HARP agency middleware . . . . . . . . . . . . . . . . 141

7.3 Order of magnitude comparison of the memory requirements of agent platforms designed for embedded applications. . . . . . . . . . . . . . 141

7.4 Summary and comparison of CubeADCS and cell-based ADCS characteristics 159

7.5 Representative microsatellite subsystems, their processing capacity, associated peripherals, and equivalent number of proteins . . . . . . . . . 161 



\section{List of Acronyms and Abbreviations}

ACL agent communication language

ADC analogue to digital converter

ADCS attitude determination and control system

AIM attitude interface module

AMS agent management system

ASIC application-specific integrated circuit

CAN Controller Area Network

CMG control moment gyroscope

COMM communication

COTS commercial off-the-shelf

DAC digital to analogue converter

DMIPS Dhrystone million instructions per second

DNA deoxyribonucleic acid

EPS electrical power system

ESA European Space Agency

FIPA Foundation for Intelligent Physical Agents

FPGA field-programmable gate array

FROM ferroelectric random-access memory

GPIO general-purpose input/output 
GPS global positioning system

HARP Hybrid Agent Real-Time Platform

I/O input/output

I2C Inter-Integrated Circuit

IC integrated circuit

IMU inertial measurement unit

JPL Jet Propulsion Laboratory

LED light-emitting diode

LEO low earth orbit

MCU microcontroller

MEMS micro-electromechanical systems

MEO medium Earth orbit

MM macromolecular machinery

MOSFET metal-oxide-semiconductor field-effect transistor

MTTF mean time to failure

MUX multiplexer

NASA National Aeronautics and Astronautics Administration

OBC onboard computer

PCB printed circuit board

PDF probability density function

ROM read-only memory

RTOS real-time operating system

SSC Surrey Space Centre

SSTL Surrey Satellite Technology Limited

TAE task allocation entity 


\section{Chapter 1}

\section{Introduction}

A traditional satellite, often weighing several tonnes and larger than a small car, can cost millions of pounds to develop and launch. However, thanks to advancements in many fields, including integrated circuits, battery technology, and composite materials, satellites smaller than a loaf of bread are now commonplace.

Developing, launching, and operating a satellite has always been an expensive exercise. Therefore, the reduction in satellite size has largely been driven by a desire to cut costs. Since access to space aboard a rocket is essentially priced per kilogram, any mass savings are valuable. However, it is not just their small physical size which distinguishes these satellites from their traditional counterparts. The extensive use of commercial components, reduced redundancy, simplified test campaigns and shortened development time lines all characterise the new class of small, low cost satellites. While these satellites continue to grow in popularity and capability, even enabling new mission concepts, the cost-cutting measures that have made them possible come with undesirable side effects. To date, one of the largest has been reduced reliability.

At first, this reduced reliability was not seen as a major concern. The first generations of small, low cost satellites were primarily educational tools, designed to give students and hobbyists hands-on experience of satellite design and operation. A few weeks of primitive on-orbit interactions were often enough to signify mission success, as the majority of the mission's goals would have been achieved by the time the satellite reached orbit.

However, as subsequent generations of small, low-cost satellites began integrating more complex and capable payloads, scientific, governmental and commercial organisations began to take an interest. Today, this new class of satellites is beginning to challenge the roles of 
traditional, large and expensive satellites, especially in the area of Earth observation. For this challenge to be successful, however, they must demonstrate a certain level of reliability.

Once outside the Earth's atmosphere, a satellite is in a position of extreme physical isolation. On-orbit maintenance is not currently a possibility, and contact with ground stations is often limited to a few minutes a day. Thus, a satellite, like a biological organism, must be self-sufficient. Both must harvest energy, recover from damage caused by the environment, and make life-preserving decisions. Since the process of evolution has resulted in biological organisms so rugged they can survive in volcanoes and ice fields, this work proposes that a satellite's system architecture should more closely resemble that of a biological organism.

Nature has always been a source of inspiration for engineers, and examples of bio-inspired design are common in everyday life. Velcro, for example, was inspired by the cockleburs which clung to an engineer's coat after a walk in the woods. Similarly, the Schmitt Trigger, which is fundamental electronic component found in almost every digital device, was invented by an engineer after studying squid nerves. In this case, the proposed system architecture is inspired by the functions within, and interactions between, the cells of a multicellular organism.

This work aims to demonstrate the theoretical reliability benefits and practical feasibility of a proposed multicellular system architecture. This architecture is composed of the Satellite Stem Cell Hardware and Hybrid Agent Real-Time Platform (HARP) middleware. Together with the development of the architecture, analytical reliability equations are derived, a multicellular system simulator is developed, and two physical multicellular systems are implemented and characterised.

\subsection{Research Motivation}

The cost-cutting techniques essential to the development of small, low-cost satellites have several negative effects on system reliability. Firstly, by aiming for small size and low weight, satellite designers are forced to minimise hardware redundancy. Secondly, by reducing development time, testing campaigns are cut short and an increased number of design flaws go unnoticed. Finally, through the extensive use of commercial, off-the-shelf (COTS) components, a satellite is put at an increased risk of radiation and temperature induced component failures. 
The trend of reduced reliability in low-cost satellites is most evident amongst CubeSats, which are a class of satellites typically weighing one to five kilograms. Out of the first hundred CubeSats to be launched, more than a quarter of industry missions and more than a third of university missions have been dead on arrival or died shortly after reaching orbit [1]. Thus, while CubeSats and other low-cost satellites provide exciting opportunities, their low reliability needs to be addressed before they can truly compete with traditional, large and expensive satellites.

Techniques to increase system reliability are well known to satellite developers. Some techniques, such as the extensive use of radiation hardened components, are designed to minimise the probability of failures. Others, such as dual-string architectures, are designed to make a system fault tolerant. While these techniques have proven to be effective on large, expensive satellites, their high implementation cost, in terms of money, man-power, weight and volume, make them poorly suited to low-cost satellites. New solutions to increased reliability are required for this new class of satellite.

Biological systems have evolved a number of techniques which aid their survivability. A number of these operate at cellular level, and it is these techniques which provide the inspiration for the research described in the thesis. By adopting similar strategies for implementation in man-made systems, this research is aimed at enhancing the reliability of low-cost spacecraft avionics.

The architecture of multicellular life is of particular interest. It differs significantly from most man-made systems. In a simplified sense, every multicellular organism starts out as a set of initially identical cells, called stem cells, which go on to specialise in specific roles within the organism through a process known as differentiation. In some organisms, this process is even reversible, allowing differentiated cells to re-differentiate, typically in response to damage to the organism. This task distribution process occurs through cellular peer-to-peer communication and without guidance from a central authority.

From a manufacturing standpoint, a system made of initially identical building blocks is well suited for low-cost, mass manufacture. From a reliability standpoint, the ability of every building block to potentially take over from any other failed building block within the system allows high priority tasks to be kept executing, even as partial failures build up. This represents the global application of functional redundancy, the implementation of which has traditionally been limited due to the functional separation and bespoke nature of traditional subsystems (e.g. the attitude control computer may be able to take over some functionality from a failed main onboard computer). 
Further potential for increased system reliability is presented by the peer-to-peer task allocation strategy, as it lacks the potential single point of failure presented by a central coordinating body (such as the main onboard computer in many traditional system designs).

However, while a theoretical, artificial, multicellular architecture promises many benefits, development of a practical system is challenging. Modularity and multi-functionality traditionally come with overheads in mass, volume and cost. Furthermore, the reliability benefits gained through the reconfigurable nature of the architecture may be outweighed by the negative effects of added module-level complexity. Thus, work is needed to determine the theoretical reliability, and practical feasibility, of an architecture based on multicellular concepts.

\section{$1.2 \quad$ Aims \& Objectives}

This research aims to increase the reliability of small, low-cost satellites by proposing, analysing and demonstrating a novel system architecture based on key principles of multicellular life.

In pursuit of this aim, the main objectives of this research are to:

1. Conduct a parametrised, empirical reliability study on low-cost satellites to identify causes of unreliability.

2. Form a set of guiding principles for the development of a robust, low-cost system architecture by studying aspects of multicellular life.

3. Based on the set of guiding principles:

(a) Develop a hardware building block, inspired by the biological cell, which will maximise reuse throughout a satellite's avionics.

(b) Develop a decentralised software architecture which will leverage the cell-based hardware to maximise system reliability.

4. Derive a set of analytical reliability equations for predicting the lifetimes of simple multicellular systems.

5. Develop a multicellular system simulator for studying complex multicellular systems and for validating the derived analytical equations. 
6. Implement a testbed multicellular system for determining practical feasibility and overheads.

\subsection{Contributions}

This research includes the following areas of novelty:

1. The conceptual development of a satellite stem cell - This work describes the inspiration behind, and development of, a bio-inspired, fault-tolerant, reconfigurable hardware building block. By focussing on the role of proteins, interfacing directly to peripherals, and having concern for implementation overheads, the satellite stem cell allows complex, practical sensor-actuator systems to be implemented using minimal additional hardware.

2. The adaption of agent computing to multicellular systems - Building on the increasingly popular field of Agent Computing, this work describes the development of a software framework which turns every satellite stem cell into an agency. These agencies operate as the artificial cells' macromolecular machinery, 'synthesising' protein agents in response to internal and external conditions. The Hybrid Agent Real-Time Platform (HARP) middlware's novelty lies in its small memory footprint, enhanced fault tolerance, and embedded, decentralised task allocation strategy.

3. The theoretical reliability analysis of multicellular systems - Through the derivation of analytical reliability equations and the development of a multicellular system simulator, trends in the reliability of multicellular systems are characterised to form a guide for future multicellular research and systems development.

4. The characterisation of practical multicellular systems - By implementing two systems based on the proposed multicellular concepts, this work extends beyond theory and proves the practical feasibility of the proposed architecture. Based on the current generation artificial cells, microsatellite-scale systems are predicted to benefit from cellularisation, while CubeSats are better suited for the single-celled strategy of 'produce many and a few will survive'. 


\subsection{Thesis Structure}

The rest of this thesis is structured as follows:

Chapter 2 introduces the concept of small, low cost satellites by giving a brief overview of their history and exciting future missions which they are enabling. Through a literature review of empirical satellite reliability surveys, their relatively low reliability compared to conventional spacecraft is highlighted. Traditional techniques for increasing reliability and their application to low-cost satellites is discussed, before coming to the conclusion that new techniques are required. Furthermore, the result of a parametric survey conducted on 159 CubeSats is presented, showing that there is no simple 'recipe-for-success'.

Chapter 3 begins with an in depth investigation of the techniques employed by unicellular and multicellular organisms, which have enabled them to flourish in even the harshest environments on Earth. From this investigation, the outline of a novel system architecture, based on the biological processes of protein synthesis, differentiation and inter-cellular communication, is presented. Further inspiration is drawn from several other fields, including bio-inspired processors, agent computing, and modular satellites, which are discussed in the remainder of the chapter.

Chapter 4 expands on the outline of the proposed multicellular architecture by describing in detail the design of an artificial satellite cell. The cell is intended to act as a generic building block, replacing unique, discrete subsystems. By mimicking several biological processes, the artificial cell is able to reconfigure to perform a large variety of tasks and interface directly to a variety of common sensors and actuators. Furthermore, the chapter explains how a system can be built up from a number of these cells, and how this architecture allows for graceful degradation in the event of failures.

Chapter 5 describes a set of middleware, named HARP, which facilitates inter-cellular cooperation through peer-to-peer communication and distributed task allocation. The middleware is based on the concept of Agent computing, and distinguishes itself from other agent platforms through its small memory footprint, real-time task execution, and fault-tolerance. The middleware's relation to the Foundation for Intelligent Physical Agents (FIPA) Abstract Architecture is described in detail, together with its unique features and additions.

Chapter 6 presents the derivation of analytical reliability equations for simple systems based on the proposed multicellular architecture. Based on these equations, several predicted 
trends in the behaviour of multicellular systems are discovered and discussed. The equations are used to compare the theoretical reliability of multicellular and traditional system architectures. Additionally, a multicellular system simulator, developed in MATLAB, is presented. It is used to simulate more complex multicellular systems, and to verify the derived analytical equations.

Chapter 7 describes the implementation of two systems based on the concepts developed in this thesis. The first system is composed of a single artificial cell, and serves as the payload interface computer of a CubeSat. The second system is testbed designed to demonstrate the full functionality of the proposed multicellular architecture. A series of experiments designed to determine the testbed's performance, overheads and scalability, are described. The chapter concludes with two case studies, which investigate the benefits and overheads of 'cellularising' traditional sets of satellite avionics using the testbed cells.

Chapter 8 concludes the work by summarising important results, highlighting key novelties and proposing areas for future research. 



\section{Chapter 2}

\section{Background}

This chapter introduces a class of small, low-cost satellites which are rapidly growing in popularity and enabling exciting new mission concepts. Thereafter, some basic concepts of reliability are introduced and their application to traditional, as well as small, low-cost satellites, is described. With the aid of a literature review of empirical reliability studies, the poor reliability performance of small, low-cost satellites to date is highlighted and discussed. A novel, parametrised study of empirical CubeSat reliability is presented, before concluding that alternative reliability-increasing techniques, better suited to the resource constrained environment of a low-cost mission, are required.

\subsection{Rise of the Small, Low-Cost Satellites}

The services provided by satellites are an integral part of modern life. Unfortunately, developing, launching and operating a satellite has always been, and still is, a very expensive business. For example, NASA's 2013 Landsat 8 earth observation satellite, which is the size of a large car and weighs $2623 \mathrm{~kg}$, has a mission cost of 855 million US dollars [2] (Figure 2.1a). Similarly, the European Space Agency's (ESA) Sentinel synthetic aperture radar satellite, which weighs $2300 \mathrm{~kg}$, was built for 280 million Euros [3] (Figure 2.1b).

However, as electronics have gotten smaller and launch opportunities have increased, new classes of smaller, lower-cost satellites have appeared. These missions employ a combination of several strategies to achieve their cost savings. Firstly, by building a physically smaller and lighter satellite, launch costs are significantly reduced. This is because launch cost is, in a simplified sense, directly related to satellite mass and typically accounts for $30-40 \%$ of 


\section{Background}
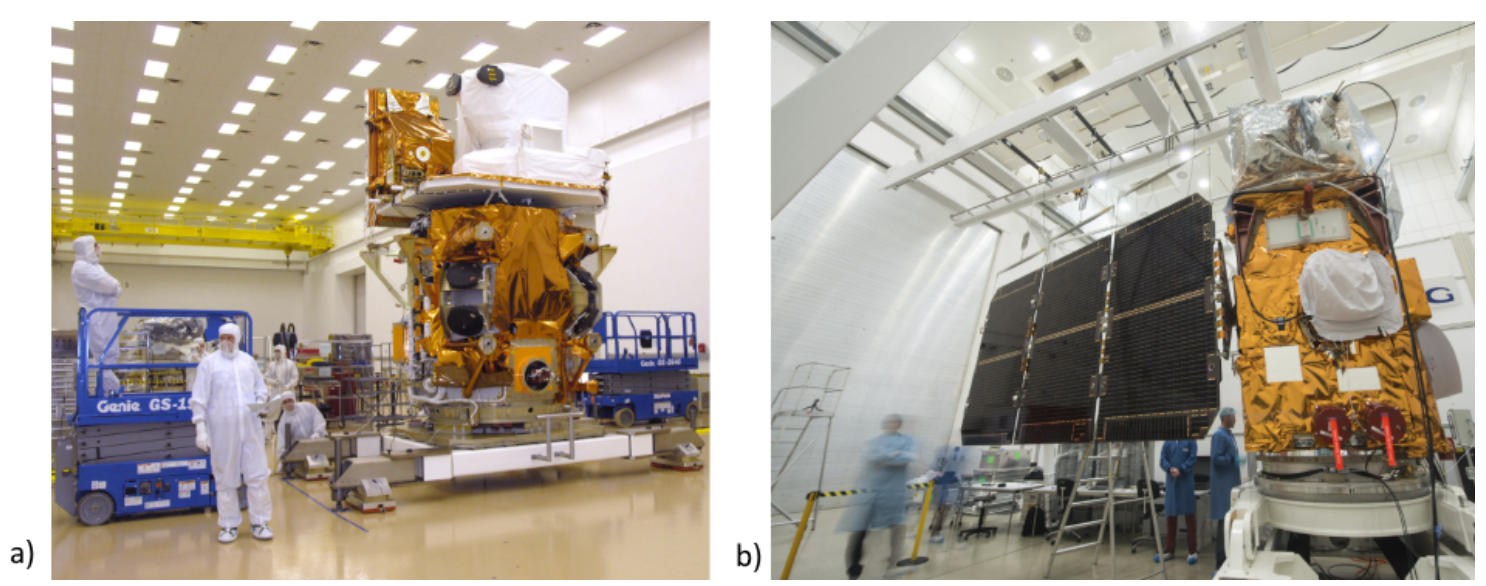

Figure 2.1: NASA's $2623 \mathrm{~kg}$ Landsat 8 satellite (a) [4], and ESA's $2300 \mathrm{~kg}$ Sentinel satellite (b) [5], undergoing testing. These satellites represent examples of the traditional 'big-aerospace' approach.

total mission expenses [6]. A further benefit of building a smaller satellite is the increased number of launch opportunities that become available through ride-sharing. Many launch providers now allow small satellites to take up any excess capacity on the launch of large, primary satellites. For example, in 2017, a single launch of the Indian Polar Satellite Launch Vehicle (PSLV) successfully placed the Cartosat 2D primary spacecraft, together with 103 secondary satellites, into orbit [7].

In addition to decreased mass, reduced development time is another major cost-saver. While traditional satellite missions can take five to ten years to develop, low-cost satellites have been developed in as little as six months. This is achieved through a combination of reduced test campaigns, descoped mission aims and the extensive use of commercial subsystems, instead of custom developments. Additionally, many development time lines are driven by available ride-share launch opportunities, which may be announced on relatively short notice.

Finally, the material cost of a satellite can be substantially reduced by using commercialgrade electronic components, rather than expensive, space-grade ones. Space-grade can be orders of magnitude more expensive because these components are extensively screened and have features such as radiation resistance or tolerance, extended temperature operating ranges, and are made of materials which are stable in vacuum. Using commercial-grade components comes with risk and will likely reduce the total lifetime of the satellite. However, this risk can be reduced by choosing components which already have space-flight heritage, and by implementing certain pre-emptive measures, such as latch-up monitoring circuits. Furthermore, because the space-industry represents such a small fraction of the market, spacegrade components often lag behind their commercial counterparts in terms of technological 
developments by several years. Thus, using commercial parts can have the benefit of reduced power consumption and improved performance.

\subsubsection{Important Examples}

The earliest small, low-cost satellites were designed by clubs of amateur radio operators, and were literally assembled in home workshops. The earliest of these satellites, named OSCAR I, was launched in 1961, barely four years after the launch of Sputnik. Three more successful OSCAR satellites followed, before AMSAT, The Radio Amateur Satellite Corporation, was formed as an educational institution which aimed to embrace and expand on the OSCAR project. These early OSCAR satellites had little intelligence and operated mainly as beacons or radio frequency transponders in aid of amateur radio hobbyists back on Earth.

In 1978, with the aid of AMSAT members, the University of Surrey in England began its UoSat microsatellite research program [8]. This program aimed to develop cost-effective techniques for the development of small satellites, demonstrate their capabilities and commercial value, and promote space education and training. UoSat-1, weighing approximately $50 \mathrm{~kg}$ and shown in Figure 2.2a, was built within 30 months and with a budget of $£ 250000$. It was the first microsatellite to feature an on-orbit reprogrammable computer, allowing it to be far more capable than its amateur radio ancestors. UoSat-1 was a huge success and led to the development of another 11 UoSat series satellites, each with an average development time of 10-12 months and a budget of 3 million US dollars.

In the midst of the program, in 1985, the university spin-off company Surrey Satellite Technology Ltd (SSTL) was founded. Having proven the capabilities of microsatellites, especially for earth observation and technology demonstration purposes, SSTL aimed to commercialise the university's microsatellite expertise. To date, SSTL has launched 50 satellites, ranging in mass from $6.5 \mathrm{~kg}$ to $440 \mathrm{~kg}$ and continues to be a world leader in the small satellite market.

In 1999, another important development in the history of small, low-cost satellites occured. Bob Twiggs of Stanford university and Jordi Puig-Suari of California Polytechnic State University proposed the CubeSat reference design [10]. CubeSats adhere to certain physical dimensions which allow them to be mated and deployed from launch vehicles using a standardised interface. The smallest CubeSat, named a 1 Unit (1U) is 10 x 10 x $10 \mathrm{~cm}$. Larger CubeSats, such as the popular $3 \mathrm{U}(10 \times 10 \times 30 \mathrm{~cm})$, can be created by expanding in 


\section{Background}
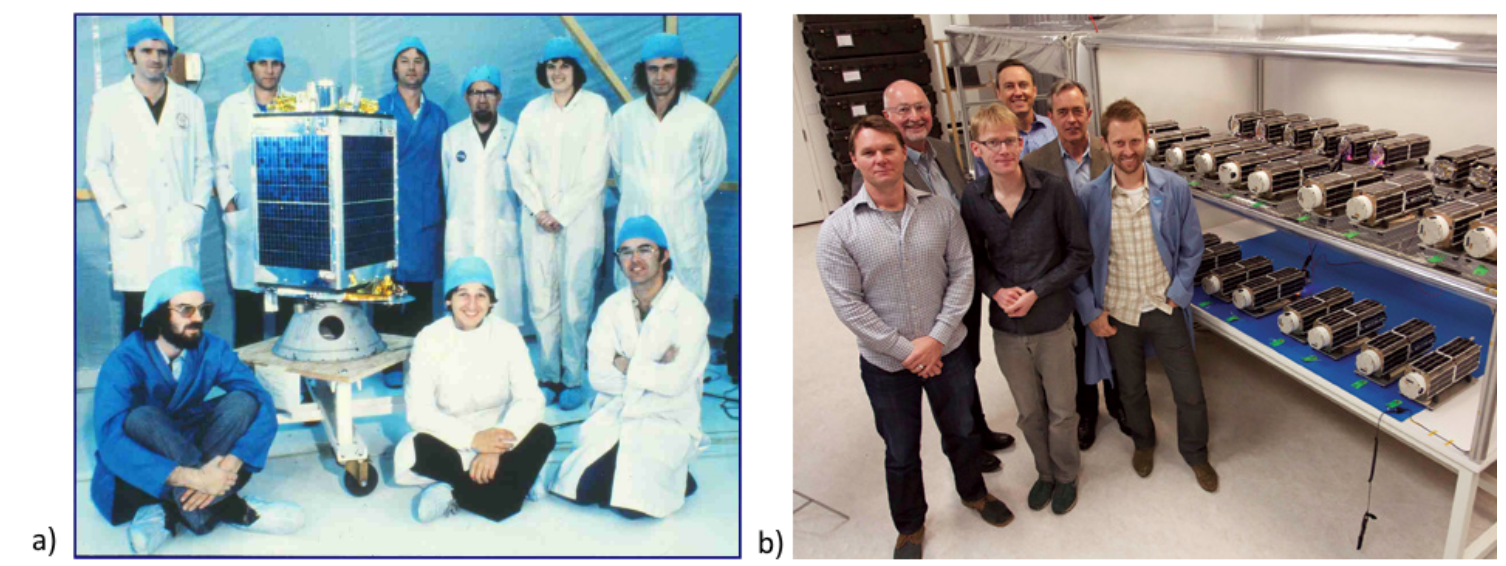

Figure 2.2: UoSat-1, the first microsatellite with a reprogrammable onboard computer 1981 (a) [8], and a number of PlanetLab CubeSats awaiting launch 2014 (b) [9]. Small, cheap satellites like these are starting to challenge the roles of traditional, multi-tonne satellites.

units of $1 \mathrm{U}$. The idea behind CubeSats was to enable graduate students to receive hands-on experience of designing, building and operating a satellite.

The first CubeSats were launched in 2003, and the CubeSat reference design quickly became a standard. Today, mid-way through 2017, more than 700 CubeSats have been launched. Arguably, the largest contribution of the CubeSat standard has been the universal launch adapter called a PPOD (several other loosely compatible variants exist). The PPOD is a spring-loaded container which typically deploys a single $3 \mathrm{U}$, or three $1 \mathrm{U}$ satellites at a time. Many launch vehicles are now compatible with PPODs, providing many additional rides into orbit. Additionally, the CubeSat standard has led to a marketplace of CubeSat subsystems. Every component, from the structure, to the attitude control subsystem and radio transceiver, is available off the shelf, which has allowed many universities and even schools to build their own satellites. However, as shall be shown in Chapter 3, building a successful CubeSat is still far from a plug-and-play process.

While CubeSats were largely an educational tool for many years, commercial, governmental and research organisations are showing increased interest. A CubeSat's small physical size makes integrating a useful payload challenging, but it also allows the manufacture and launch of large constellations. For example, the commercial organisation Planet Labs owns the largest earth observation constellation of satellites in history, being composed of several hundred 3U CubeSats (Figure 2.2b). Each CubeSat is able to image the earth with 3-5 meter resolution, and the constellation as a whole has the ability to image the whole Earth every day [11]. 
The Von Karmen Insitute for Fluid Dynamics (VKI), a Belgian research institute, currently has a constellation of approximately $502 \mathrm{U}$ CubeSats orbiting the Earth as part of its QB50 program [12]. The QB50 constellation aims to monitor the Earth's upper atmosphere in many locations simultaneously. Each CubeSat was built by a different organisation, from across the world, and carries a set of instruments supplied by VKI. The QB50 project is likely a precursor to many future distributed sensing experiments, which have become feasible with the advent of small, low-cost satellites.

\subsubsection{The Future of Small, Low-Cost Satellites}

Small, low-cost satellites have grown substantially in capability since the early OSCAR and UoSat projects. For example, the UoSat-1 camera produced images of just 256 x 256 pixels with a ground resolution of $2 \mathrm{~km}$, while the similarly sized Carbonite- 1 microsatellite, launched in 2015, provides images with a $1 \mathrm{~m}$ ground resolution. Similarly, UoSat's downlink was limited to 1200 bits per second, while the transmitters of Carbonite-1, operating in Xband, can downlink at 80 megabits per second [13][14].

As their capabilities keep increasing, small, low-cost satellites are enabling a variety of new mission concepts. These include:

- Large constellations for distributed sensing and increased revisit times

- In-orbit assembly of larger structures, such as telescope mirrors

- Motherships which release smaller, specialised satellites once in orbit

In addition to the Planet Labs earth observation and QB50 atmospheric monitoring constellations, a good example of a planned mega-constellation is the SpaceX internet project. The project aims to supply internet to any point on Earth by launching 4425 small, low-cost satellites into orbit at an altitude of approximately $1200 \mathrm{~km} \mathrm{[15].}$

The Autonomous Assembly of a Reconfigurable Space Telescope (AARest) mission aims to demonstrate the assembly of large structures in orbit. As shown in Figure 2.3, it will consist of a mothership, which holds the focal plane, and several CubeSats, each of which contain a portion of the main mirror. Once on orbit, the CubeSats will detach from the mothership and autonomously reattach in different configurations. If successful, the mission could pave the way to much larger mirrors and antennas being assembled out of small satellites in the future [16]. 


\section{Background}

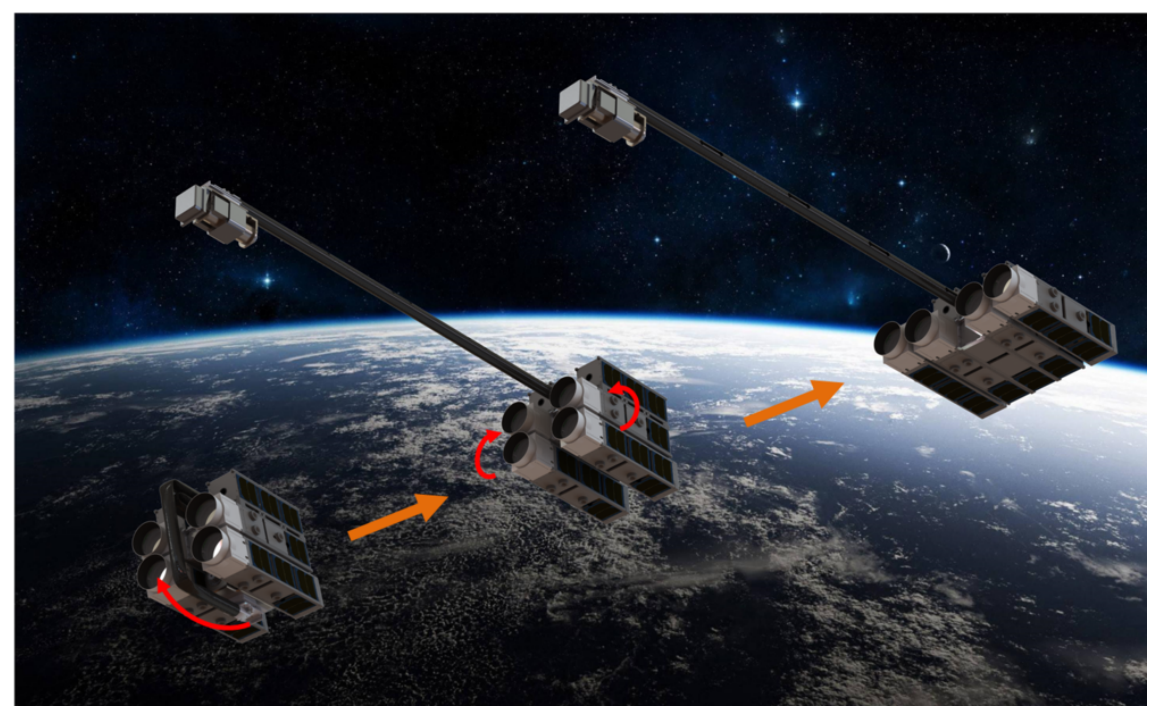

Figure 2.3: The AAReST mission aims to demonstrate the assembly of a large mirror composed of small satellites on orbit cite [16].

The RemoveDebris mission will deploy two CubeSats to act as targets for a variety of active, orbital debris removal techniques. The first CubeSat is relatively dumb and will be targeted by a 'net-cannon', which aims to capture the CubeSat before reeling it back in. The second CubeSat contains a capable attitude determination and control system, whose measurements will be compared to those made by an experimental, vision-based targeting system on the mothership [17].

These three examples are just a few of the small, low-cost satellite missions that are expected to be launched over the next couple of years. Figure 2.4 shows a progression of the number of sub-50 kg satellites that have launched each year, and the predicted number to be launched over the next few years. Since the SpaceX megaconstellation is still just a proposal, it is not included in the prediction. Nevertheless, nearly 2400 nano and microsatellites are expected to be launched before 2023 .

As small, low-cost satellites continue to grow in capability and popularity, they will continue to challenge the roles of traditional, 'big-aerospace' satellites, while enabling new mission concepts. However, in order to be useful and competitive in the future, small, low cost satellites will require a certain level of reliability, which has, on average, been lacking to date. 


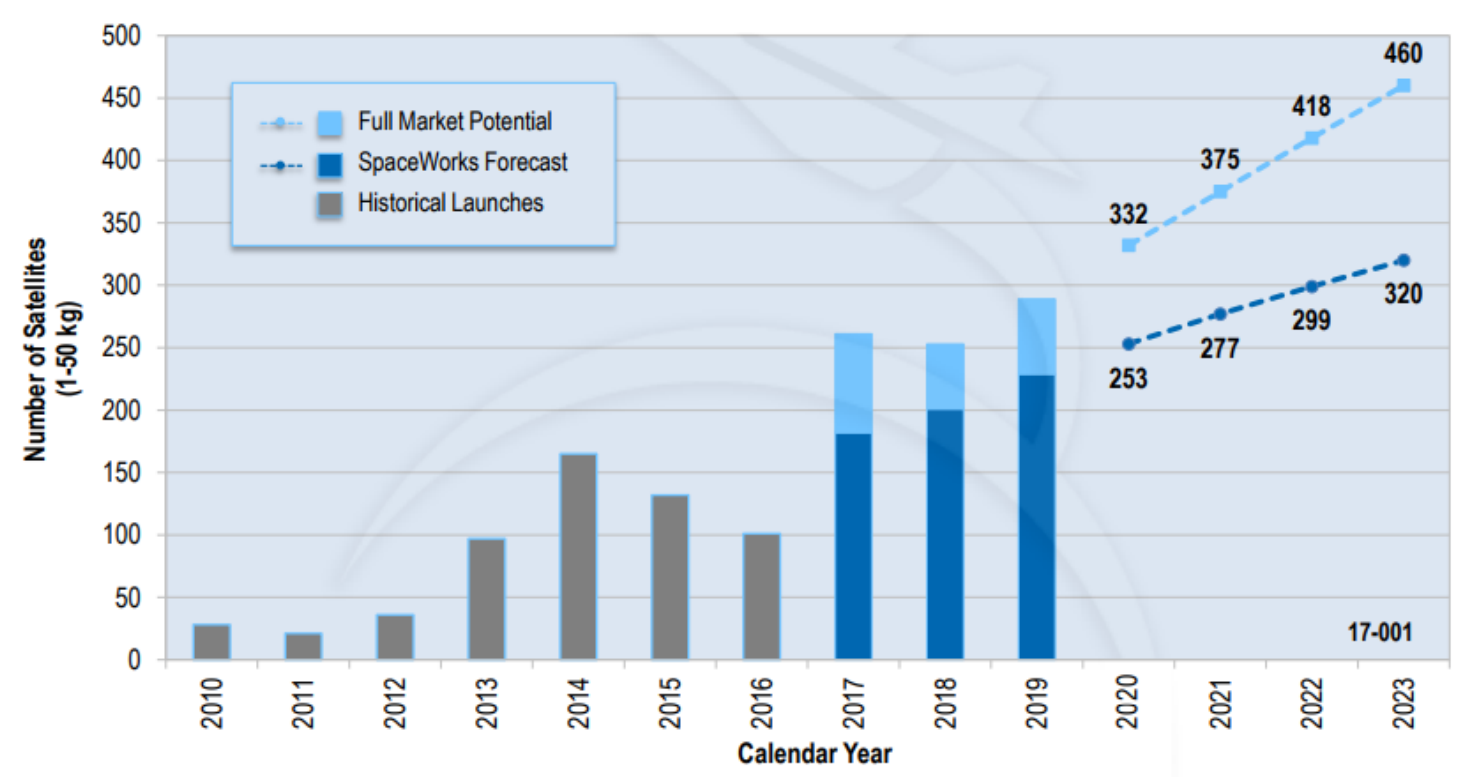

Figure 2.4: SpaceWorks' prediction of the number of sub-50 kg satellites to be launched over the next few years [18].

\subsection{Reliability Overview}

Reliability is defined as the probability of performing without failure, a specific function, under given conditions for a specified period of time [19].

Failures can be divided into three categories, namely permanent, transient, and parametric. A permanent failure, also known as a catastrophic failure, is unrecoverable without external maintenance. This maintenance may include the replacement of parts, or the manual reloading of firmware. Transient failures are intermittent and either resolve themselves, or are resolved by the system itself. Examples of recovery include a processor restarting itself, or a seized mechanism shaking itself loose. Parametric failures do not result in complete loss of functionality. Instead, they cause a degradation in system performance. Parametric failures are common as components age and ware out. Examples include a degradation in solar panel output due to radiation dose, and an increase in current consumption of a mechanism as its lubrication is expended. This research is mainly concerned with permanent failures.

Early electronic systems based on relays and vacuum tubes were notoriously difficult to make reliable [20]. Vacuum tubes were the primary cause of unreliability, having to be replaced 5 times more often than any other component [21]. The rise of semiconductor components in the 1950's partially alleviated this problem by allowing vacuum tubes to be replaced with more reliable, solid-state components. However, the issue of reliability became 


\section{Background}

a major concern again in the 1960's with the dawn of the space age. Satellites and probes are very expensive to send into space and cannot be serviced once launched; therefore it is very desirable for them to be highly reliable. Reliability of the systems involved in manned space flight is even more essential as a failure in these systems can put human lives at risk.

Research on reliability has continued to gain importance as electronic systems have become more integrated into our daily lives. For example, a modern car contains in excess of 50 MCUs which must operate reliably to avoid putting human lives in jeopardy [22]. These include anti-lock brakes, airbags, power steering and cruise control.

\subsubsection{Fault Avoidance and Fault Tolerance}

Traditionally, techniques for increasing system reliability followed one of two philosophies: Fault avoidance or fault tolerance.

Fault avoidance attempts to minimise sources of unreliability to prevent faults from occurring during system operation. Typical examples include using high quality and screened components, perfecting assembly and integration processes, performing extensive testing, and carefully controlling the system's operational environment. 'Over-designing' is another common technique, which involves designing components to withstand conditions far in excess of what is expected to be seen during normal operation.

Since no system can reasonably be expected to operate completely fault free for the duration of its lifetime, fault intolerant design aims to decrease the system's probability of failure to an acceptably low value. This probability of failure is assessed using known or predicted failure rates of individual components. In addition, periodic preventative maintenance is often employed to improve reliability. To assist maintenance, the system may have automatic fault detection and notification abilities. However, purely fault intolerant systems have no ability to repair themselves.

Fault avoidance is popular in the space industry. Examples include:

- Using radiation-hardened components.

- Performing assembly and integration in cleanrooms.

- Over-designing and over-specifying with respect to voltage, current, temperature etc.

- Extensive test campaigns including thermal-vacuum and vibration. 
However, some aspects of the fault-intolerant philosophy are difficult to apply to satellites. For example, on-orbit maintenance is largely impractical to date, and many components are not available in radiation-hardened versions.

The second approach to achieving reliability, known as fault tolerance, attempts to cope with failures as they arise. Fault tolerant designs try to assure reliability through protective redundancy. Faults are expected to occur, whether caused by the environment, operator or flawed design. Protective redundancy can be classified as either passive or active. Passive redundancy is very similar to the fault-intolerant concept of over-designing. For example, several of the cables supporting a suspension bridge can fail before the bridge fails.

Active redundancy can be implemented in four basic ways: hardware, software, information and time. Hardware redundancy involves adding additional or duplicate hardware components. Hardware redundancy is described in more detail in Section 2.2.2. N-version programming is an example of redundancy applied in software. $\mathrm{N}$-version programming involves creating two or more pieces of software independently which satisfy the same initial specifications. The independent pieces of software are run in parallel with identical inputs to verify their output. Information redundancy includes techniques for error detection and correction such as Hamming codes. These techniques automatically detect and try to correct errors in a data stream. Finally, time based redundancy, in its simplest incarnation, performs every action multiple times in succession to ensure the action is performed correctly. A basic example would be transmitting every bit of information twice.

Upon experiencing a failure, an actively redundant system is said to recover to either failoperational or fail-safe states. A fail-operational system can recover automatically to a fully operational state. In contrast, a fail-safe system will recover to a safe, but reduced operational state, while it awaits human intervention to enable full recovery.

Generally, all forms of redundancy require more resources (power, volume, mass) than a system designed without redundancy. Therefore, instead of aiming for fully fault tolerant system, designers may aim for a partially fault tolerant system. Such a system continues to operated after failures occur, but possibly with reduced performance. This concept is also known as graceful degradation, and, while not ideal, is often better than having the system fail completely.

An example of graceful degradation can be seen in computer hard drives. A hard drive can automatically detect damaged sectors on its disks and automatically avoids writing to those sectors in the future. Besides a reduced memory capacity, the user of the hard drive will notice no difference in its operation. An example of graceful degradation in the space 


\section{Background}

industry is satellite solar panels. Most solar panels are designed with bypass diodes. Bypass diodes allow current to bypass a damaged solar cell. Without bypass diodes, damage to a single cell could disable the whole panel. Again, the only difference in performance of the panel after damage to a cell has occurred will be a reduced power generating capacity.

\subsubsection{Hardware Redundancy}

The most elementary form of hardware redundancy is cold, or backup, redundancy. When a fault occurs in a component of a system with no fault tolerance, maintenance staff may decide to replace the faulty component with an identical spare. In a fault tolerant system with cold redundancy, spare components are automatically switched in to replace faulty ones. Therefore, the system must have a series of spare components readily available and already connected to the system. All the spare components are turned off and consume no power until they are switched in. This has the added advantage of preventing wear on the spare components until they are needed. There may be a delay and interruption to service while the spare is switched in. To minimise the delay, hot redundancy may be used. In a hot redundant system, the spare components are switched on and are running in parallel with the primary components. However, the spare components' outputs are isolated from the system until the primary component fails. The disadvantage of hot redundancy is that the spare components are subject to the same wear as the primary components even while they are not being used.

A more sophisticated form of hardware redundancy is called modular redundancy. Modular redundancy makes use of a number of identical components (or modules) whose outputs are compared using a voting circuit. The voting circuit decides which output to forward to the rest of the system. The individual modules are highly synchronised and receive the same inputs at the same time. If only two modules are used, called Dual Modular Redundancy (DMR), and they have differing outputs it is not obvious to the voting circuit which module is faulty. Therefore, modular redundancy works best if an odd number of modules are used. Triple Modular Redundancy (TMR), making use of three modules, is common. A faulty output from one of the three modules is easily detected by noticing that it differs from the other two modules. In this case the voting circuit will ignore the faulty unit's output and give the majority output. If the faulty unit continues to give erroneous output it may be permanently turned off. Modular redundancy allows faults to occur in modules with minimal impact to the system. Unlike cold redundancy, there is usually no interruption while faulty units are removed from the system. However, modular redundancy requires all modules to 
be powered on and running at the same time, increasing power consumption. The voting circuitry also adds complexity and is a potential single point of failure. However, the voting circuitry is usually designed to be simple and reliable.

A third form of redundancy, called 1:N redundancy, uses a single backup for multiple components. The backup can take on the function of any one of a number of primary components. This form of redundancy requires less volume than either backup or modular redundancy, making it attractive for use on small satellites. The biggest challenge in implementing 1:N redundancy is designing the backup unit. The backup unit must be able to perform the function of any one of the primary components. Unless the primary components all have very similar functions, the backup unit will ultimately be very complex. Routing signals to the backup unit may also pose a challenge.

Related to $1: \mathrm{N}$ redundancy is the concept of degeneracy. Degeneracy refers to a partial overlap in the functions provided by different components. A system with degeneracy requires multifunctional components. These components usually perform distinct functions but can, under certain conditions, perform the same functions, effectively making them interchangeable. For example, the SUNSAT satellite has a microprocessor dedicated to OBC tasks and a microprocessor dedicated to attitude determination and control system (ADCS) tasks. However, if the ADCS processor fails, the OBC processor can take over its critical tasks [23]. This is also an example of graceful degradation as the satellite can still function albeit with reduced functionality.

Designing with degeneracy instead of redundancy can save resources as there is no need for space-wasting backup components. However, as with 1:N redundancy, designing the multifunctional components of a degenerate system can be challenging. Components are likely to become more complex as they become more multifunctional. This added complexity may lead to increased volume, weight and power consumption, while simultaneously decreasing reliability.

\subsection{Satellite Reliability}

In this section, references are made to basic reliability modelling concepts, such as reliability functions and failure distributions. Readers unfamiliar with these concepts may want to read Section 6.1 Introduction to Analytical Reliability Analysis, first. 


\section{Background}

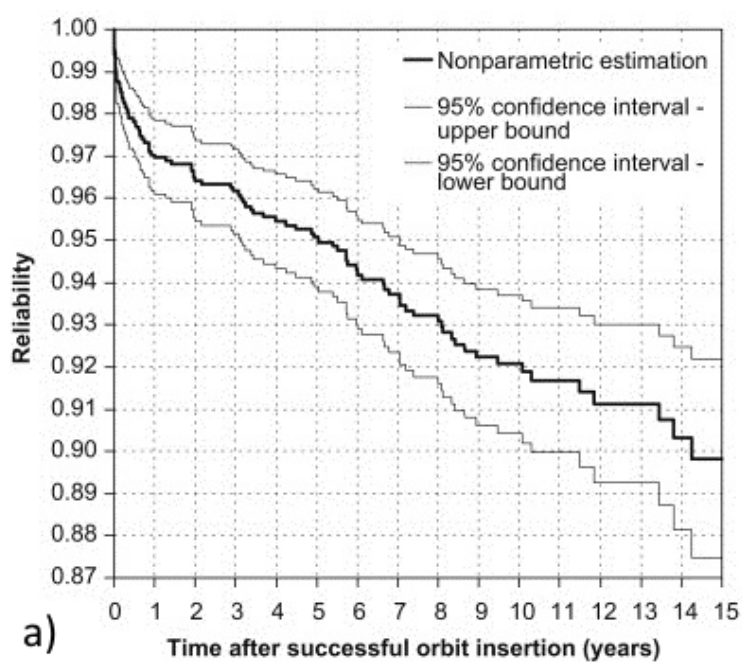

b)

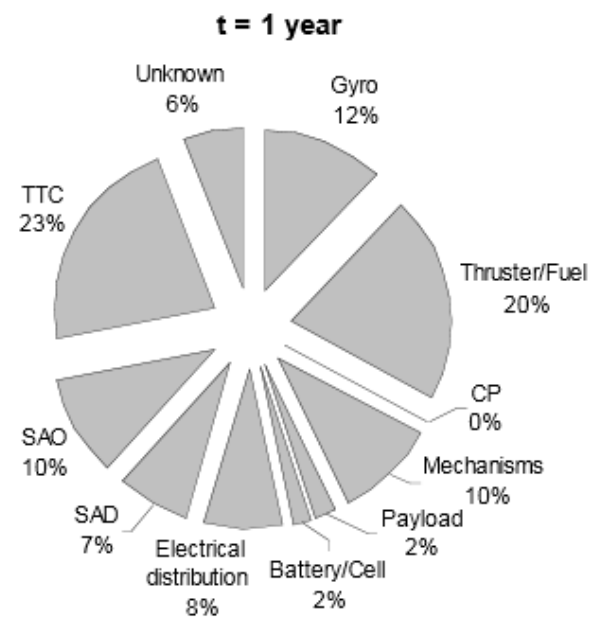

Figure 2.5: Traditional satellite reliability based on empirical failure data of 1548 satellites (a) and the most common causes of failure within the first year (b). Reproduced from [24].

Satellites have traditionally been very expensive to design and launch and cannot typically be serviced once on orbit. For these reasons, satellite designers usually put a large amount of effort into ensuring the reliability of their satellites. This effort has largely paid off, as shown by Castet and Saleh in a survey of 1548 Earth-orbiting satellites launched between January 1990 and October 2008 [24]. A statistical reliability analysis, based on a Kaplan-Meier estimator, was performed on collected empirical failure data. The resultant reliability plot is shown in Figure 2.5a. The Kaplan-Meier technique has the ability to deal with incomplete and censored lifetime data. If a satellite survived past its design lifetime or beyond October 2008 its failure date was censored (meaning its reliability is no longer considered past its design lifetime). According to the graph, approximately $3 \%$ of the satellites surveyed failed during their first year on orbit. The raw survey data, published in the paper, shows that out of 1548 satellites, only four were dead on arrival in orbit (or died within the first day).

A steep decrease in satellite reliability during the first year on orbit can be seen in the data. The authors of the study fit a Weibull distribution with $\beta=0.3875$ and $\omega=8316$ (years) to the data. With a shape parameter less than one, this Weibull Distribution is characteristic of infant mortality. Infant mortality is often caused by design faults which were not caught during testing.

Castet and Saleh also investigated the reliability of individual subsystems by noting which subsystem was responsible for each failure. Figure $2.5 \mathrm{~b}$ displays the fraction of failures (occur-ring within the first year on orbit) caused by each subsystem. The largest contributors are the tracking, telemetry and command subsystem (TTC) (which is a combination of 


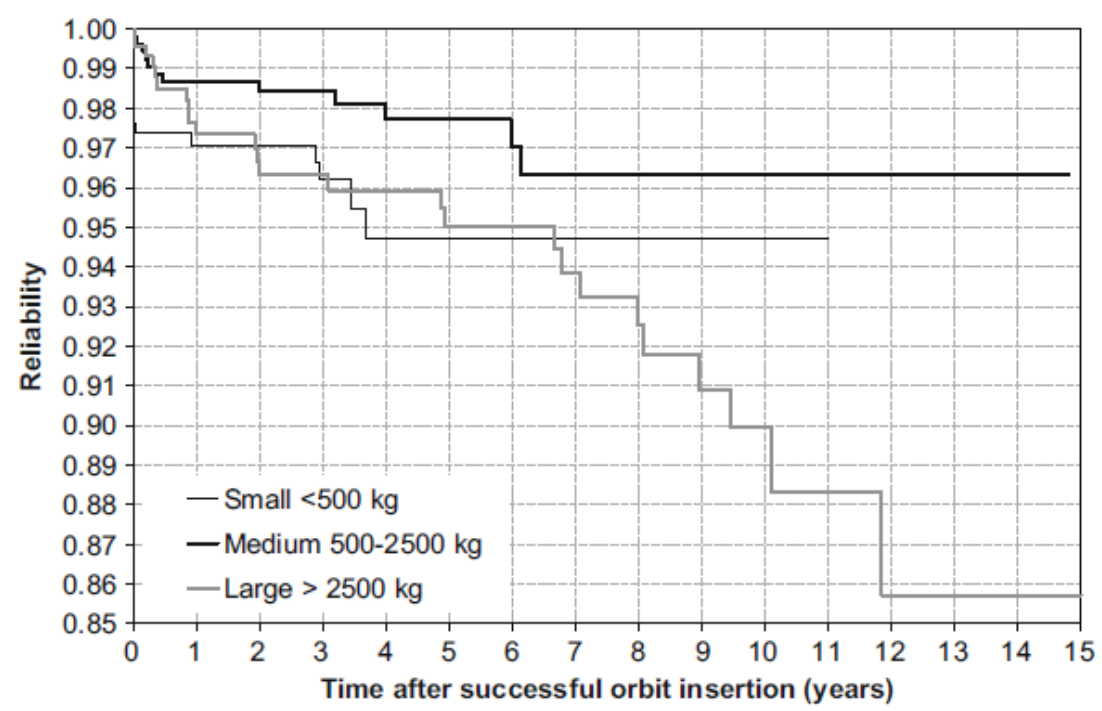

Figure 2.6: Empirical reliability plots for three mass classes of satellites. Reproduced from [25].

onboard computer and communication subsystems), the propulsion subsystem, and gyroscopes.

\subsubsection{The Poor Reliability of Low-Cost Satellites}

In comparison to the average satellite, small, low-cost satellites have shown very poor reliability to date. This is highlighted by four empirical surveys. It is important to note that budget information is rarely available for satellite missions, partly as a result of commercial companies not releasing the information, and partly because the funding of such missions can be complex and come from multiple sources. Therefore, in the remainder of this section, low-cost is associated with low mass. As described in Section 2.1, this is a reasonable assumption to make, as mass is directly related to launch cost and the availability of low-cost ride-share opportunities.

The first study, produced as a follow-up paper to Castet and Saleh's survey, was performed by Dubos and investigates whether satellite reliability can be related to satellite size [25]. The same 1548 satellites from their previous paper were split into three groups according to mass: small (0-500 kg), medium (500-2500 kg) and large (>2500 kg). Once again a Kaplan-Meier estimator was used to generate the reliability plots shown in Figure 2.6.

Several important trends in the reliability plots were noted by the authors. Firstly, the small satellites drop significantly in reliability during the first six months on orbit. They appear to 


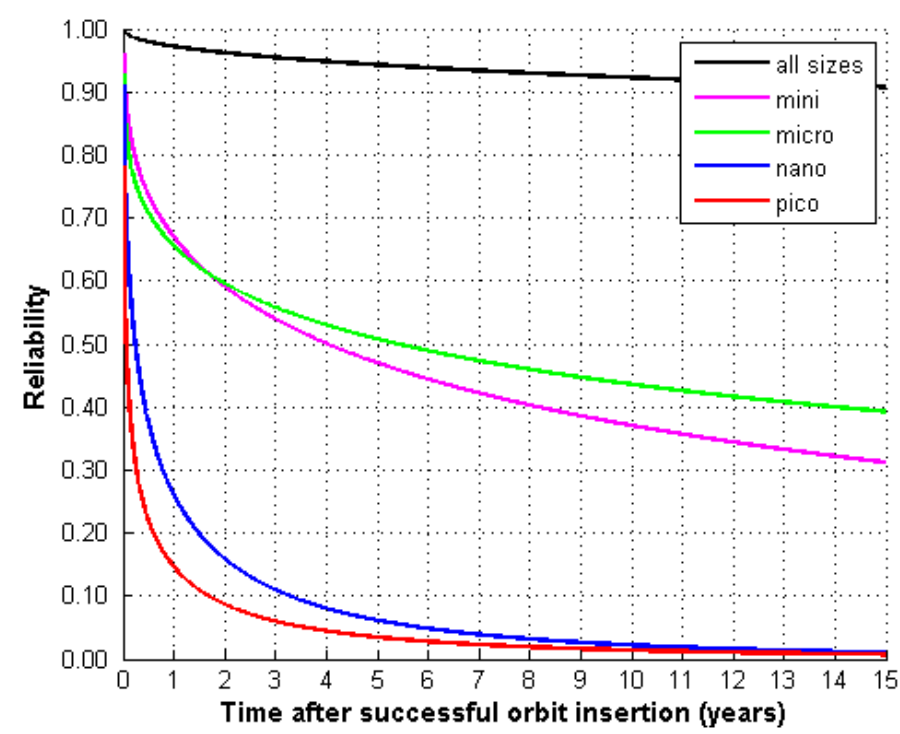

Figure 2.7: Empirical reliability plots of different classes of small satellites, based on Guo, Monas and Gill's survey of 222 satellites launched between 1990 and 2010 [26]

suffer more from infant mortality than satellites from the medium and large categories. In addition, small satellites exhibit another significant drop in reliability after three years on orbit. A second trend that can be seen in the graph is the wearing-out of large satellites. The constant reliability of small and medium satellites seen after approximately 4 and 6 years, respectively, is an artefact of the analysis. Most of these satellites will have reached their design lifetimes by then, meaning they were removed from the study, and no reliability data exists for longer durations. However, large satellites are often designed for longer lifetimes and their increased failure rate after approximately 7 years can, at least partially, be attributed to mechanical and environmental wear out of components. The study concludes that amongst the satellites studied, small satellites had the highest infant mortality rate.

The second study, by Guo, Monas and Gill [26], is based on a satellite database of 222 small satellites $(<500 \mathrm{~kg}$ ) launched between 1990 and 2010. The satellites were divided into categories based on mass: pico (0-1 kg), nano (1-10 kg), micro (10-100 kg) and mini $(100-500 \mathrm{~kg})$. A Kaplan-Meier estimator was used to generate a reliability plot for each category, shown together on one plot for comparison purposes in Figure 2.7. The Figure also includes the reliability plot of the 'average' satellite, as determined by the Castet and Saleh study. In contrast to the 'average' satellite first year failure rate of $3 \%$, the survey showed that approximately $35 \%$ of micro and mini-satellites and $70 \%$ of nano and picosatellites failed within a year. 


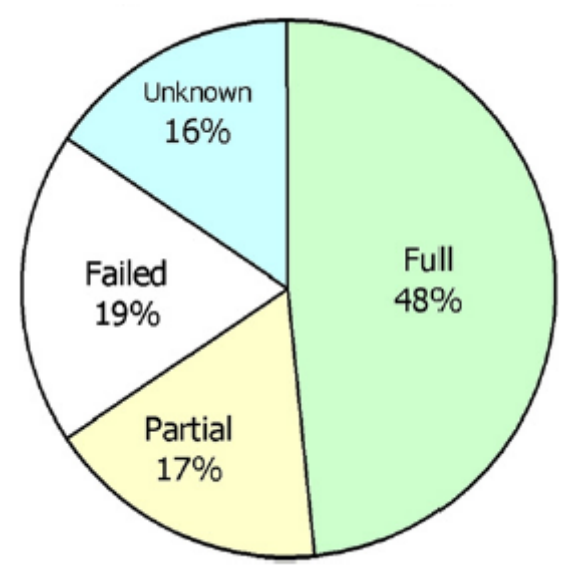

Figure 2.8: Mission success rates of a group of 94 nano satellites $(<10 \mathrm{~kg})$ launched between 1957 and 2009. Reproduced from [27].

The same study made two further findings. Firstly, it found that the TTC subsystem and electrical power subsystem were the least reliable of the investigated subsystems. This partially supports the results reported by Castet and Saleh. The study also looked at whether design lifetime had any impact on reliability. The 222 small satellites were divided into three groups according to their designed lifetime: less than five years, more than five years and unknown. They found that satellites with longer design lifetimes suffered the least from infant mortality, while those from the unknown category suffered the most. This can be explained by the fact that an unknown design lifetime often implies that limited thought has been given to the subject by the satellite designers.

The third small satellite reliability study is a paper by Bouwmeester and Guo, which investigates the success rates of nano-satellites launched between 1957 and 30 July 2009 [27]. 94 satellites with masses less than $10 \mathrm{~kg}$ are used in the study. A pie chart of the mission outcomes is shown in Figure 2.8. The paper does not define what exactly was counted as mission success, however, it normally implies completion of the primary mission objectives. Of the surveyed satellites, $48 \%$ achieved full mission success, $17 \%$ achieved partial success, $19 \%$ failed outright, and the otcomes of the remaining $16 \%$ is unknown. The authors believe that the majority of the satellites from the unknown category were probably failures, as mission publicity often rapidly dies down after a failure. Including these satellites, the failure category grows to $35 \%$.

Finally, Swartwoudt presents a study based on the first 100 CubeSats [1]. Today, CubeSats make up the majority of nano-satellite sized missions. By the start of 2014, more than 190 CubeSats had been launched. The first CubeSat was launched in 2003 and the hundredth 


\section{Background}
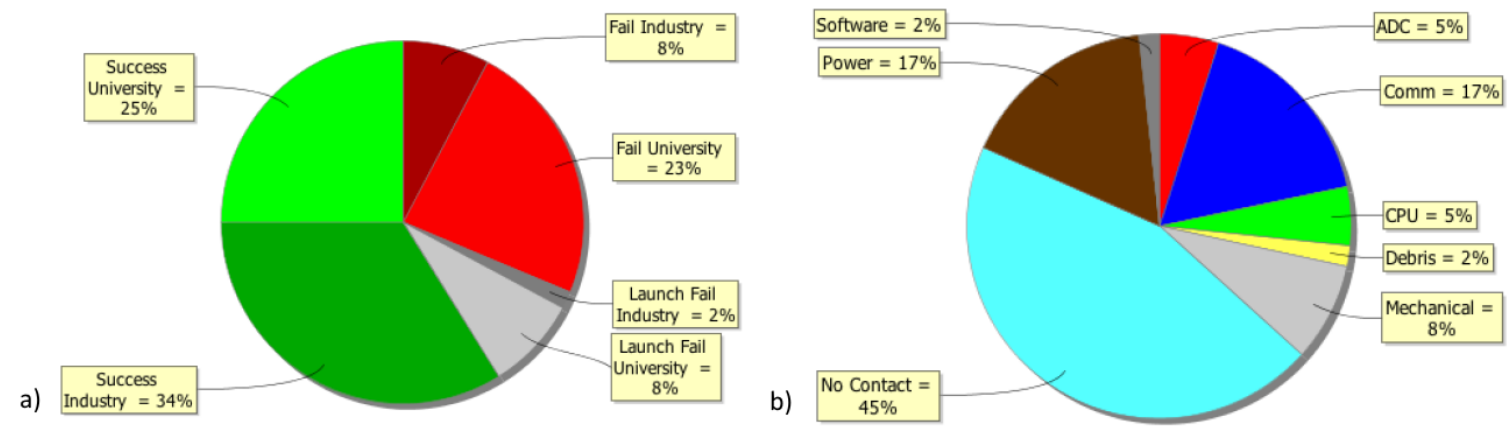

Figure 2.9: Mission success rates of the first 100 CubeSats (a), and the primary causes of failure (b). Reproduced from [1].

CubeSat was launched in 2012. Among the statistics looked at by Swartwout are mission success rates and causes of failure. Pie charts reproduced from Swartwout's paper can be seen in Figure 2.9a.

According to Figure $2.9 \mathrm{a}, 31 \%$ of the surveyed CubeSats failed early (excluding launch failures). Swartwout's paper considers a mission a failure if the mission operators publicly declared it as such, or if the satellite lasted less than 60 days in orbit. A figure of $34.4 \%$ failures (rescaled from $31 \%$ to include only successfully launched CubeSats) is similar to that found by Bouwmeester and Guo. While missions from industry fared better than those from universities, missions from both sectors showed high failure rates.

Figure $2.9 \mathrm{~b}$ shows the fraction of failures caused by each subsystem. The largest group, labeled no contact, contains those missions which were never heard from after deployment. Swartwout examined these missions more closely and came to the conclusion that this group of failures can be classified as failures in functional integration. Swartwout argues that many of these missions were let down by insufficient functional level testing. Problems that could have been caught by functional level testing include: unreliable harnessing, insufficient power generation and unrecoverable processor errors.

After problems in functional integration, the next most common causes of failures are related to the communication and power subsystems. This agrees with the findings of Guo, Monas and Gill.

These four studies highlight the large difference in reliability seen to date between traditional satellites and the new class of small, low-cost satellites. But why does this reliability gap exist? Based on the literature reviewed, the causes of unreliability can be attributed to several factors. 
Firstly, intrinsic satellite mass and volume limitations inhibit the implementation of hardware redundancy. Since hardware redundancy eats into mass and volume budgets without adding science value to the mission, may small, low-cost satellites are based on single-string designs. A further problem with single-string designs is that determining the cause of failure is often difficult or impossible as a single failure can cause the satellite to stop communicating with the ground. Once the satellite has stopped communicating, operators are left to hypothesise at the cause of failure. Without a known cause of failure it is challenging to come up with a recovery plan or to design out the cause in future satellites.

Secondly, small mission budgets necessitate the use of commercial-off-the-shelf (COTS) components instead of radiation hardened, space-rated, and screened components, which can cost orders of magnitude more. Once in space, electrical components, and especially complex integrated circuits, are susceptible to a variety of undesirable and damaging radiation and temperature induced effects. Amongst others, these include single event upsets, latch-ups and gate ruptures. In addition to their low price, COTS components are attractive to designers as a large variety of useful ICs have been developed for terrestrial applications. While these ICs simplify circuit design effort, their complexity makes their behaviour difficult to predict in unusual environments such as in orbit.

The third cause for unreliability amongst small, low cost satellites is time pressure. While traditionally satellites have taken several years to design, it is not uncommon for satellites of this new class to be designed and built in less than a year. The time pressure is caused by limited mission budgets and the ride-share nature of many of their launches. Ride-share launches occur when a large primary satellite's launch vehicle has excess capacity, allowing small satellites to 'piggy-back' on the launcher. While ride-share launches offer significant cost savings, their unpredictable availability and dependence on the primary satellite's readiness make planning around them difficult. Time pressure can lead to design mistakes, skipped reviews, and reduced test campaigns.

Fourth, inexperience, especially amongst university mission teams, leads to design and integration mistakes. Universities represent one of the leading small, low-cost satellite developers. They are developing satellites largely as a tool to train engineering students. However, universities, especially upon embarking on their first satellite missions, are often ill-equipped and too inexperienced to complete the missions successfully. Facilities, such as cleanrooms and thermal chambers, are difficult to procure and engineering students are inexperienced, even in terrestrial application design. Fortunately, many of these projects have completed their primary goal of giving students hands-on engineering experience, even before the satellites reaches orbit. 


\section{Background}

Finally, related to inexperience, is overconfidence. The CubeSat standard has led to the availability of a variety of off-the-shelf subsystems, which may give the illusion of plug-andplayability. Even the subsystems themselves may not seem overly complicated compared to terrestrial designs. However, it is simple to overlook the issues that are presented by the launch and orbit environments. Terrestrial designers take for granted maintenance access, convective heat dissipation, a steady power supply and the ability to hit reset when the software stalls. Despite growing popularity, building a satellite is still a challenging undertaking.

\subsubsection{CubeSat Survey - Finding a Recipe for Success}

A large variety of CubeSat missions have been completed to date, with mission outcomes ranging from complete success to dead on arrival in orbit. These missions are all an important source of information in the study of small, low-cost satellites. With a typical mass of less than five kilograms and a budget measured in tens of thousands of British Pounds (excluding launch), CubeSats fall towards the lower extreme end of the small, low-cost satellite spectrum. In addition, since many CubeSats are university research projects, academic publications make information on their design and performance readily available. This is in contrast to commercial satellites, where insightful design and reliability information is rarely made public. Thus, in an attempt to find a 'recipe for success', a paramaterised survey of 159 CubeSat missions was conducted. While other studies have focussed on external factors, such as class (university/commercial/governmental) or mission type (earth observation/communication/science), this study examines the design of the satellites themselves One of the main sources of mission status information for the survey was Swartwout's online CubeSat database [28]. However, a large number of additional sources, including mission websites and academic publications were consulted. The full database, with sources, is given in Appendix A.

The first step in conducting the survey was to define and determine levels of mission success. The levels of mission success are given below (note that missions which ended due to launch failure were not included in the survey):

1. Dead on arrival in orbit.

2. At least one beacon or downlink packet received by any ground station.

3. Successful uplink achieved.

4. On-orbit mission objectives partly completed. 
5. On-orbit mission objectives fully completed.

Next, a number of parameters were identified for comparison against mission success rates.

First is the level of implemented redundancy. Measured out of a maximum of three, this parameter specifies how many of the core subsystems are redundant. Core subsystems include the onboard computer (OBC), electrical power system (EPS) and communication subsystem. Any form of redundancy is allowed, as long as mission success is still achievable after primary failure. For example, if another subsystem can take over from a failed OBC, the OBC is counted as redundant. However, a separate radio beacon does not mean that the communication (COMM) system is redundant as the beacon cannot take over from a failed TTC subsystem. It is expected that higher levels of redundancy will result in higher satellite reliability.

Second is the number of COTS subsystems. Again measured out of three, this parameter specifies the number of core subsystems which are COTS components. COTS CubeSat subsystems are readily available from a number of suppliers such as Gomspace, Clyde Space, Innovative Solutions in Space (ISIS) and CubeSpace. COTS subsystems generally have flight heritage and their use is expected to increase satellite reliability.

The third parameter is a measure of the attitude determination and control system's complexity:

\section{No ADCS.}

2. Passive attitude control (e.g. compassing, gravity gradient).

3. Active magnetic control using magnetorquers.

4. Active magnetic control coupled with a momentum wheel.

5. Full 3-axis pointing control.

It is expected that satellites implementing simpler attitude control schemes will prove to be more reliable.

The fourth parameter specifies one of three system architecture types:

1. Star architecture. The OBC connects to subsystems via separate, dedicated interfaces.

2. Single-master bus architecture. All subsystems are slaves to the $\mathrm{OBC}$ on a shared bus. 


\section{Background}

3. Multi-master bus architecture. Subsystems can freely instantiate communication with one another over a shared bus.

Star architectures commonly employ universal asynchronous receiver-transmitter (UART) links, single bus architectures are most commonly based on an Inter-Integrated Circuit (I2C) bus, and CAN buses are popular in multi-master architectures.

The fifth and final parameter is development time. It is measured from project start to launch day. Technically, this is not a correct measure of development time as launch delays are common. However, teams experiencing launch delays may have added time to work on software and possibly to perform more testing. CubeSat development time varies significantly in duration, from less than a year to more than five years. A longer development time may be an indication that more thought was put into the design process and that more thorough testing was performed on the satellite before launch. This argument leads to the prediction that satellites with a longer project duration will show higher levels of mission success.

Each parameter was investigated individually by splitting the set of 159 surveyed satellites into groups based on their characteristics. For example, when looking at the ADCS complexity parameter, the satellites were split into one of five groups based on the characteristics of their ADCSs. The mission success rates within each of these groups were then compared to one another. The results are depicted graphically in Figures 2.10-2.12.

As Figure 2.10a shows, the relationship between level of redundancy and mission success rates is largely as expected. Mission success rates increase as the number of redundant subsystems increase from zero to two. All satellites with two out of three redundant subsystems achieved at least two way communication. Interestingly, beyond this level of redundancy, mission success rates are seen to decrease again slightly. This decrease is likely due to the complexity of implementing a satellite with such high levels of redundancy.

An example CubeSat with fully redundant core subsystems which performed poorly is TurkSat-3USat [29]. TurkSat-3USat is a 3U CubeSat and the second nanosatellite mission from Istanbul Technical University. The core subsystems are all functionally redundant, each consisting of a primary COTS solution and a secondary custom developed solution. The primary EPS, OBC and COMM subsystem are manufactured by Clyde Space, Pumpkin and AstroDev, respectively. TurkSat-3USat was launched in April 2013, but operated for less than a day before falling silent. The development of TurkSat-3USat faced many problems, including RF system heat dissipation and grounding issues which led to loss of the engineering model, and I2C bus disconnections and data loss. In addition, despite the full redundancy of the core subsystems, the power bus lacked redundancy and recovery options. 


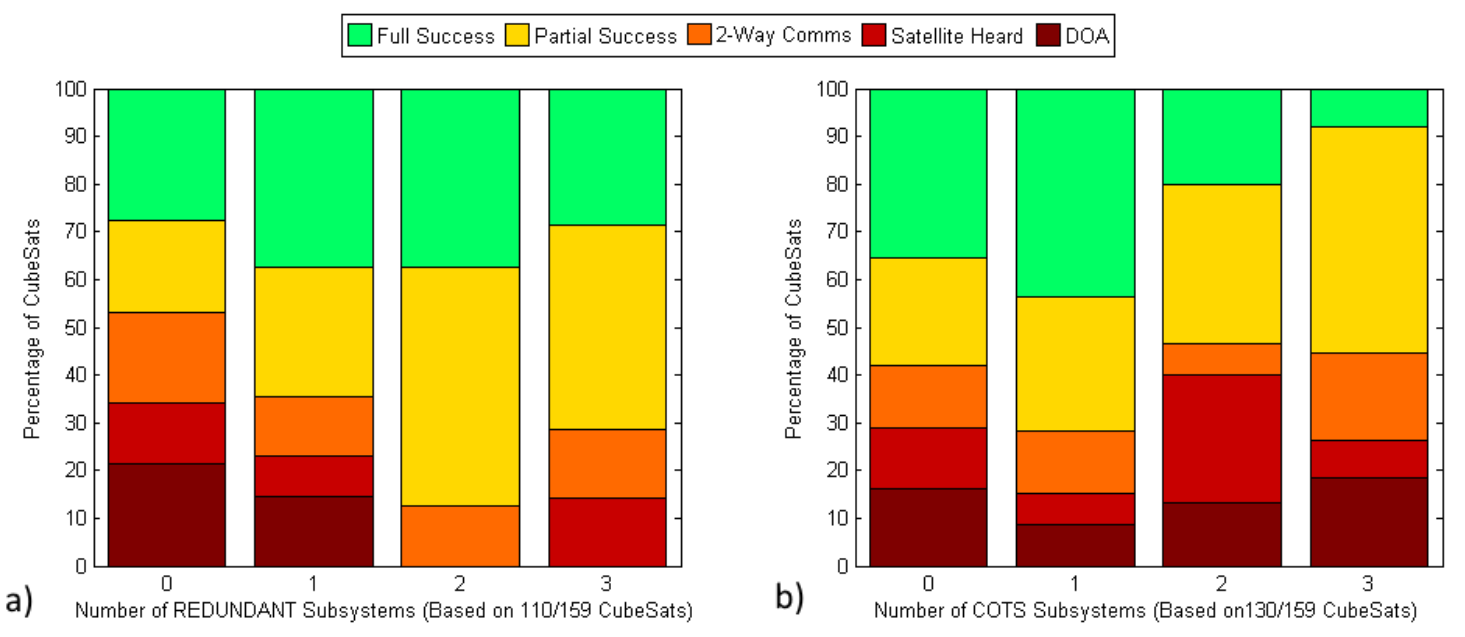

Figure 2.10: CubeSat mission success rates versus number of redundant core subsystems (a), and number of COTS core subsystems (b).

TurkSat-3USat demonstrates that introducing subsystem redundancy is not sufficient without considering interfaces and inter-subsystem interactions.

Figure 2.10b shows an unexpected relationship between number of COTS subsystems and mission success rates. While CubeSats with one COTS subsystem (55\% full mission success) generally fared better than those without any COTS subsystems (35\% full mission success), the trend does not continue for CubeSats with two or three COTS subsystems. In fact, the trend reverses, as CubeSats with two COTS subsystems achieved a full mission success rate of only $20 \%$, and those with three only $7 \%$. This inverse relationship is unexpected and is likely due to problems in satellite integration. Inexperienced teams may be tempted to buy COTS subsystems due to their apparent plug-and-playability, leading to insufficient system level testing, as mentioned by Swartwout [1]. Teams that opted to use fewer COTS subsystems likely have a better understanding of their satellites, or at least had enough experience to know which subsystems were beyond their ability to design.

An example of a CubeSat mission which employed all COTS core subsystems, but still failed to perform well on orbit, is HiNCube [30]. HiNCube is a $1 \mathrm{U}$ CubeSat built by undergraduate and masters students of Narvik University College in Norway. Its core subsystems are a GOMSPACE NanoCOM U480 transceiver, GOMSPACE NanoPower P-Series EPS, and a Pumpkin MSP430 OBC. HiNCube was launched in November 2013, but was never heard from in space. Despite the use of all COTS core components, the team experienced several integration issues, including switched-off subsystems hanging the central I2C bus, severe electromagnetic interference from the COMM subsystem, and ground support equipment 


\section{Background}

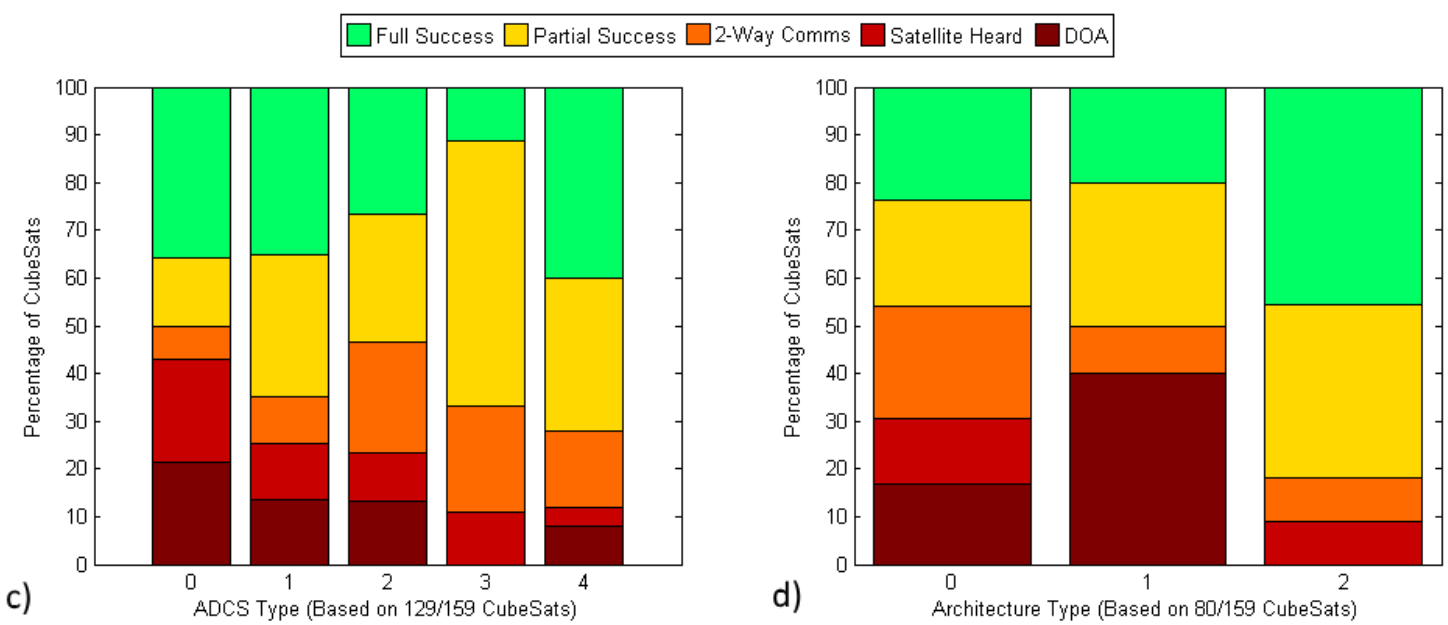

Figure 2.11: CubeSat mission success rates versus ADCS complexity (a), and system architecture type [0-star, 0-single-master bus, 0-multi-master bus] (b).

ground loops causing subsystem damage. HiNCube demonstrates the dangers of assuming COTS components will simply plug together to form a reliable satellite.

The relationship between ADCS complexity and mission success rates, shown in Figure 2.11a is also unexpected. Initially ignoring the most complex subsystem category (4), two trends can be seen. Firstly, the number of very poorly performing CubeSats is seen to decrease with increasing levels of ADCS complexity. $20 \%$ of the CubeSats with no attitude control systems were dead on arrival, while not a single CubeSat with a Type 3 ADCS was dead on arrival. An explanation for this trend is based on team experience. First time satellite builders are likely to forgo complex ADCSs, but still suffer from poor performance due to other design and implementation mistakes. In contrast to the first trend, the fraction of CubeSats achieving full mission success decreases with increasing ADCS complexity. This is easier to explain, as missions requiring more complex ADCSs usually also have more complicated missions to perform.

Comparing mission success rates to system architecture types reveals the advantage of a Type 2, multi-master, architecture (Figure 2.11b). In comparison to the CubeSats featuring this type of architecture, all of which were at least heard from in orbit, $40 \%$ of the Type 1 CubeSats were dead-on-arrival. While both Type 0 (star) and Type 1 (single-master bus) architectures rely on the $\mathrm{OBC}$ to facilitate data transfers between subsystems, the Type 1 architecture has the added vulnerability of bus hangups. A failure in following the communications protocol, or a failed or misbehaving subsystem, can all potentially lead to the bus being held-up, stalling all subsystem communication. Type 1 architectures based on the I2C bus have become the most popular choice for CubeSats as a large variety of MCUs have 


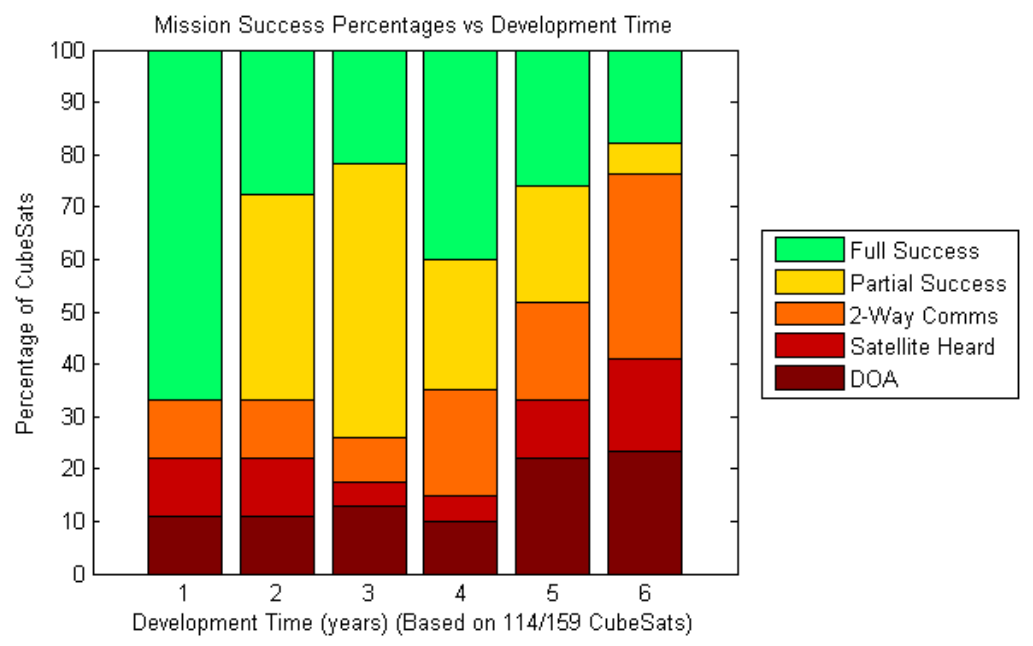

Figure 2.12: CubeSat mission success rates versus development time. The last category (6) includes missions which took longer than six years to develop.

built-in I2C interfaces, and a large range of COTS subsystems are available based on this interface. Unfortunately, the I2C standard is not very strictly defined, leading to a variety of slightly different implementations. This has led to a number of reported $\mathrm{I} 2 \mathrm{C}$ bus problems on CubeSats [29][30][31][32].

In contrast, only 11 of the surveyed CubeSats are based on multi-master architectures, which are most commonly implemented using a CAN bus. In comparison to an I2C bus, a CAN bus consumes more power and far fewer MCUs support it natively. This is likely why, despite its empirical reliability advantages, Type 3 architectures have not been common to date.

Finally, Figure 2.12 shows a comparison between development time and mission success rates. The overall trend of longer development times leading to reduced mission success rates is unexpected. $65 \%$ of the surveyed CubeSats that were deveoped in under a year achieved full mission success. In comparison, less than $20 \%$ of the CubeSats which took six or more years to develop could match this performance. CubeSats with 6+ year development times that performed poorly in orbit in clude the University of Montpellier's ROBUSTA, which only briefly emitted weak signals [33][34], and the Thomas Jefferson High School for Science and Technology's TJ3Sat, which was never heard from in space [35]. Long development timelines often indicate inexperienced development teams and can lead to problems with knowledge transfer as people join and leave the project during its development. For example, TJ3Sat was worked on by more than 50 students during its 7 year development.

Short development timelines are possible for experienced teams that can build on heritage designs. For example, CalPoly has developed eight CubeSats, with launches occurring 


\section{Background}

on average every 18 months since 2006 [36]. However, in lieu of experience and heritage designs, short development timelines can be tackled by having a stable core team of designers, simplifying mission aims, and making sensible use of COTS subsystems.

The results of this survey can be summarised as follows:

1. Including redundant subsystems has a positive effect on reliability.

2. Mistakes in integration are common and cannot be prevented by buying commercial subsystems.

3. Missions requiring more complex ADCSs are less likely to succeed.

4. Non-redundant, single-master bus architectures should be avoided.

5. Longer development time does not necessarily equate to more reliability.

Thus, while this survey uncovered interesting trends, it largely highlights the fact that mission success is the result of a complex interaction between many factors, several of which, such as team experience, development infrastructure, and mission complexity, are difficult to measure. Additionally, it shows that simple reliability assumptions, such as COTS subsystems are more reliable, or more redundancy is always better, are not always true, especially when considered in isolation. Thus, the survey does not provide a simple "recipe for success".

\subsection{Summary}

This chapter began with an introduction to the new class of small, low cost satellites. It highlighted their growing capabilities and popularity, and described several exciting future mission concepts. However, through a review of empirical reliability surveys, it then went on to highlight their poor on-orbit reliability seen to date.

A discussion of traditional techniques for improving reliability followed. These techniques follow one of two philosophies, namely: fault avoidance, or fault tolerance. Fault avoidance attempts to prevent faults from occuring in the first place, and includes techniques such as component screening and extensive test campaigns. Fault tolerance attempts to cope with failures as they occur, and contains most traditional forms of redundancy. However, the large overheads imposed by these traditional techniques, in terms of mass, volume and budget was highlighted, making them poorly suited for implementation on small, low-cost satellites. 
In an attempt to gain further insight into the causes of unreliability amongst small, low-cost satellites, a parametric reliability survey of 159 CubeSats was conducted. The results showed many counter-intuitive trends, including CubeSats with longer development times displaying reduced reliability. While some advice for future CubeSat developers could be drawn from the results, they largely show that there is no simple recipe for success.

To improve the reliability of small, low-cost satellites, new techniques, which minimise overheads, are required. 



\section{Chapter 3}

\section{Sources of Inspiration}

The first part of this chapter introduces the remarkable robustness of biological life. It describes the techniques used by unicellular and multicellular life to achieve this robustness, and proposes that certain characteristics of multicellular life, if successfully replicated in a man-made system architecture, could provide increased satellite reliability while minimising overheads. These features are combined into the outline for novel, distributed system architecture.

Further inspiration is drawn from a variety of research fields, including bio-inspired processors, agent platforms, and modular spacecraft concepts. A literature review of important works from these fields is presented, and the expected novelties of this work, with regards to each field, is highlighted.

\subsection{Bio-Inspiration}

In many ways, a satellite can be compared to a biological organism. Both have the ability to survive independently in their environments, with ways of harvesting energy, hardware which continues to function without external maintenance and the ability to make life-preserving decisions. In the biological case, organisms must face environmental and biological hazards, such as UV radiation and viruses. Similarly, a satellite must endure hazardous temperature swings and constant attack from charged particles and micrometeorites. Since biological life has achieved an impressive level of robustness, flourishing even in the most inhospitable parts of the planet, alternative techniques for increasing reliability are often found by studying characteristics of biological life. 


\subsubsection{Unicellular Life}

A cell is the smallest independently replicating unit of life. The earliest cells appeared at least 3.8 billion years ago [37]. Since then, cells have become the building blocks of all life on Earth.

All living organisms are composed of one or more cells. The earliest forms of life were single celled organisms based on simple cells called prokaryotes. Single celled organisms include most bacteria, unicellular fungi and unicellular algae. While single celled organisms perform all the functions necessary to maintain life, including moving, feeding, producing waste and reproducing, the majority never grow beyond microscopic size and remain relatively simple.

Cells have evolved to include various mechanisms to increase robustness. These include: genetic redundancy, DNA repairing mechanisms, special adaptions to extreme environments and the ability to transfer genes. However, as shall be explained, these techniques are essentially analogous to the well-known techniques of functional hardware and software redundancy, EDAC algorithms, special radiation hardened components and on-orbit reprogramability.

Genetic redundancy allows certain genes in an organism to become damaged without causing any noticeable effects. In fact, several studies with bacteria, worms and mice have shown that only a fraction of their genes are essential [38]. The set of non-essential genes contain non-functional genes and redundant genes. Redundant genes can compensate for damaged genes, and have been shown to exist in many organisms [39]. For example, a biochemical reaction within a cell may be redundantly catalysed by two distinct proteins generated from different genes. Functional redundancy may also exist at higher functional levels within a cell. For example, a study showed that $28-38 \%$ of the chemical reactions involved in the metabolism of the microbe E.coli can be inhibited without lethal results [40]. These forms of redundancy can be compared to hardware/software functional redundancy in a man-made system.

Cells have the ability to repair their own DNA if it gets damaged through a form a information redundancy. DNA damage is caused by both normal cellular activities and environmental factors. Human cells experience up to a million molecular lesions per day, each of which threaten the integrity of the DNA [41]. The information redundancy manifests itself as in the double helix structure of the DNA. Each DNA molecule is composed of two identical strands. If damage occurs to only one strand, the other strand can be used as a template to repair the damage [42]. If damage occurs to both strands, cells use more complex and error 


\subsection{Bio-Inspiration}
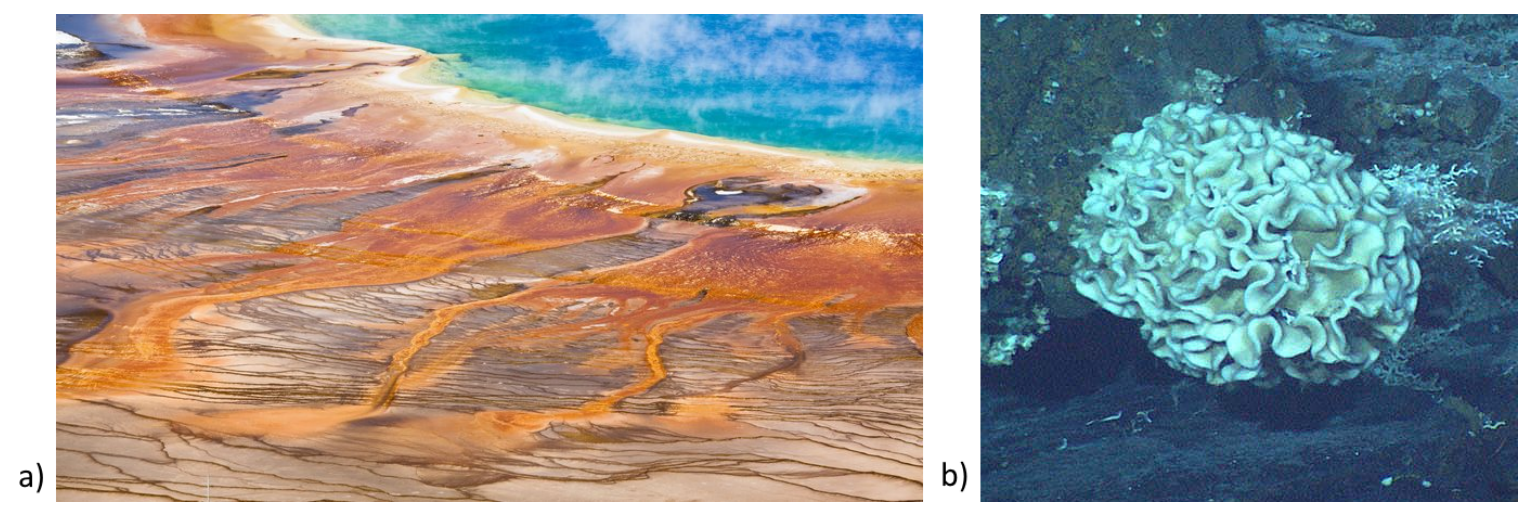

Figure 3.1: The bright colours of Yellowstone National Park's geysers are caused by extremophiles thriving in the 74 degree Celsius water (a) [43]. Giant single-celled extremeophiles have been found living in the harsh conditions at the bottom of the Mariana Trench, $11 \mathrm{~km}$ below the surface (b) [44].

prone techniques to attempt repair [41]. These techniques are comparable to error detection and correction (EDAC) schemes used by man-made systems.

The most inhospitable locations on Earth are characterised by extreme temperatures or pressures, high levels of radiation or poisons, low levels of moisture, or other hazardous conditions. To survive in under such conditions, organisms known as extremophiles have evolved special adaptions. For example, to function in the extremely hot environment near a hydrothermal vent, thermophile cells utilize special heat tolerant proteins in place of ordinary proteins in cellular activities [45]. Similarly, electronic components have been designed especially for the hazardous environment of outer space. Such components are typically designed to operate over wide temperature ranges ( -40 to +85 degrees Celsius) and can be made resistant to the effects of radiation. However, space-rated, radiation-hardened components usually cost orders of magnitude more than their commercial counterparts.

Horizontal gene transfer, which occurs within the same generation, is an alternative to gene transfer through reproduction. Usually an organism's genes remain constant throughout its lifetime. However, certain organisms, chiefly bacteria, have shown the ability to to transfer genes between themselves. Such cells can benefit by taking up foreign genetic material and altering their own genes. Horizontal gene transfer is thought to be a significant cause of developed drug resistance. This occurs when one bacterial cell develops a drug resistance through a random mutation, and passes the mutated gene to neighbouring cells. The ability to reprogram a satellite on orbit is the man-made analog to horizontal gene transfer. A satellite that can be reprogrammed on orbit could, within reason, be modified to cope with unexpected environmental changes or design mistakes. 


\section{Sources of Inspiration}

Single celled organisms are still abundant on Earth, despite having existed for billions of years. The reliability-increasing techniques seen in use by these organisms have well-known analogues amongst man-made systems. Unfortunately, as discussed in Chapter 2, these techniques typically incur large overheads, making subsystem-level implementations impractical and unaffordable for small-low-cost satellites. Nevertheless, as will be described in the following sections, when implemented at a cellular level within a multicellular architecture, these techniques play a crucial role in giving a system multiple levels of redundancy.

An important note to add here is that unicellular organisms have another survival strategy. Their large numbers allow many individual organisms to die without compromising the species as a whole. Thanks to small, low-cost satellites, the 'build many and accept a few losses' strategy is becoming feasible to satellite mission designers, too. For example, Planet Labs has a constellation of more than 100 CubeSats which are used for Earth observation [11], while SpaceX is planning a constellation of several thousand small satellites for internet distribution [46]. Other projects have suggested making satellites as small as a single PCB [47], or a single IC [48], to enable large swarms to be deployed. In either case, the loss of a few satellites will have minimal impact on the overall mission outcomes. While this strategy is interesting to study in itself, it shall not be the focus of this work.

\subsubsection{Multicellular Life}

At some point in the Earth's past multi-cellular organisms began to appear. There are currently three major theories as to how this occurred [49]. The symbiotic theory states that multi-cellularity arose from a symbiotic relationship amongst a group of different unicellular organisms. As the organisms became more and more dependent on each other for survival, gene transfer eventually merged the organisms into a single multicellular organism. A second theory, called Cellularisation, states that the origin of multi-cellularity may have been cells with multiple nuclei. Such cells still exist, supporting the theory. The final theory, Colonial Theory, states that multi-cellularity may have arisen from the symbiotic behaviour of a group of cells of the same species. While there is still a lot of debate about how the first multicellular organisms evolved, biologists agree that it has happened independently several times in different branches of the tree of life [50]. This suggests that there are definite evolutionary advantages to multicellular life. Today, all large, complex organisms, including animals and plants, are multicellular.

Multicellular life has a fundamentally different architecture to unicellular life, and demonstrates alternative ways of achieving robustness. As various aspects of multicellular life are 
Table 3.1: Number of specialised cell types versus total cell count in several organisms

\begin{tabular}{|l|l|l|}
\hline Organism & Number of Cell Types & Typical Total Cells \\
\hline Placozoa & 4 & few thousand \\
\hline Hydra & 15 & $50-70$ thousand \\
\hline Jellyfish & 22 & millions \\
\hline Human & 411 & $3.72 \times 10^{13}$ \\
\hline
\end{tabular}

detailed in the sections that follow, the alternative reliability-increasing techniques, and their potential for being ported to man-made systems, are discussed.

\subsubsection{Cells versus Subsystems}

Multicellular life is based on cooperating cells. This arrangement allows individual cells to specialise and take on specific tasks through a process known as differentiation. Typically, a multicellular organism starts life as a single cell called a Zygote. A Zygote is totipotent, meaning it can differentiate into any other form of cell. As the number of cells within the organism starts to increase due to cellular division, cells begin to differentiate based on location within the organism and many other factors. Humans contain approximately 411 differentiated cell types, including nerve cells, fat cells and red blood cells [51].

It may be tempting to describe a traditional, subsystem-based satellite as having a multicellular architecture with each subsystem representing a differentiated cell. However, this analogy has three flaws. Firstly, the basic building blocks of a satellite are typically unique, discrete subsystems, while the basic building blocks of a multicellular organism are, in a simplified sense, a set of initially identical cells. While a satellite's subsystems are generally designed from the ground up to perform a specific set of functions, each cell initially has the potential to perform any function. It is only through the process of differentiation that the cells take on specific function sets.

Secondly, in biological organisms the number of differentiated cell types and extent of differentiation typically scales with the total number of cells, as shown in Table 3.1 [51][52]. An organism with four cells representing four different subsystems is not a good analogy to what is seen in nature. Such an organism would be less robust than a single celled variant as the death of any one of its cells would lead to the death of the whole organism. Instead, the cells in a simple organism composed of only a few cells would all have fairly similar functionality. 
Finally, as is described in detail in the following section, the forms of redundancy seen in multicellular organisms, while familiar, vary significantly from their traditional, man-made counterparts.

\subsubsection{Natural Redundancy}

Through cell cooperation and the process of differentiation, multicellular organisms benefit from three forms of redundancy.

Firstly, the lifetime of a multicellular organism is not dictated by individual cell lifetimes. Cells are constantly dying and being replaced without any significant effect on the organism. This is a form of cold redundancy. Because cells have the ability to self-replicate, a multicellular organism has a large supply of spare parts. Unfortunately, man-made systems do not yet have the ability to self replicate from raw materials obtained from the environment. Thus, practical versions of cold redundancy require maintenance access, or sufficient excess margins for all spare parts to be housed within the system from the very start. This is not an attractive option for small, low-cost satellites.

In addition to cold redundancy, multicellular organisms display modular redundancy. Table 3.1 showed a comparison between the total number of cells and the number of unique cell types in various multicellular organisms. There are typically orders of magnitude more cells than there are cell types, implying that every task is being performed with significant redundancy. If a few cells are misbehaving, their effects are essentially overpowered by the correctly behaving majority. This is comparable to a massive voting circuit, where the majority wins. Modular redundancy, such as TMR arrangements, are common in high reliability systems. However, due to size, man-made systems cannot practically match the sheer numbers involved in the biological version. In addition, unlike in the biological case where the voting process is essentially analogue and not reliant on an arbiter, in digital systems there is always the problem of how to make the voting circuitry, itself, reliable.

Finally, as the process of differentiation is not necessarily final, multicellular organisms can display functional redundancy. Some biological cells retain the ability to de-differentiate and re-differentiate throughout their lifetimes. Such additional phases of differentiation are typically triggered by damage to the organism and occur in the area surrounding the damage. For example, a zebra fish can regenerate after losing up to $20 \%$ of its heart. Fully differentiated cells in its heart de-differentiate before re-differentiating and proliferating to restore the missing tissue [55]. Similarly, damage to the cells in the lens of a newt's eye will 


\subsection{Bio-Inspiration}

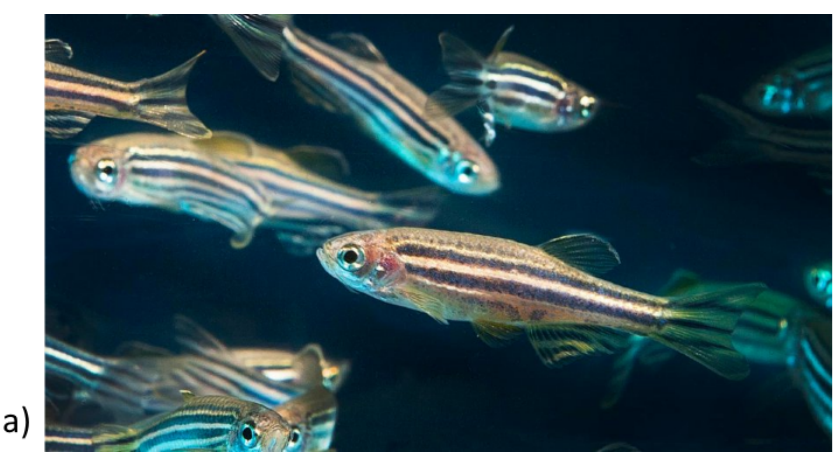

b)

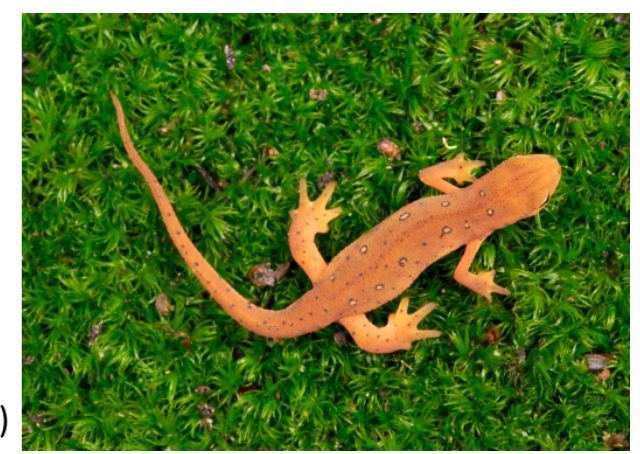

Figure 3.2: Zebrafish (a) and Newts (b), can recover from cell damage through cell re-differentiation. This is a form of redundancy only possible in multicellular organisms. Image credit [53][54]

cause nearby cells to de-differentiate and re-differentiate to replace the damaged lens cells [55]. If the potential for cell division and multiplication is disregarded, the process of cell re-differentiation can be viewed as a form of graceful degradation. Cells performing less essential tasks have the ability to take over from damaged cells which were performing critical tasks. Intrinsic, system-wide functional redundancy is rarely, if ever, seen in man-made systems. Therefore, it is worth exploring this concept in more detail.

\subsubsection{A Closer Look at Differentiation}

Understanding the process of differentiation and how cells with the same DNA can specialise into performing wildly different tasks, begins with an understanding of the importance of proteins. Proteins are complex molecules which can aid in and perform a variety of chemical and physical processes. Many cellular functions are performed by proteins, so it is important for a cell to be able to produce its own set of proteins. The instructions for this are stored in the cell's DNA and are known as genes. The process of using a gene to produce proteins is called gene expression.

The process of going from DNA to proteins is split into two steps, named transcription and translation. Transcription is performed by a large molecule known as RNA polymerase. RNA polymerase creates copies of particular sections of DNA. These copies are single stranded, unlike DNA, and are called messenger RNA strands. The second step of gene expression, translation, is performed by a large molecule called a ribosome. The ribosome synthesises a particular protein according to information in the messenger RNA (mRNA), from raw materials absorbed from the environment. The details of transcription and translation are not important here. However, it is important to note that these two processes can be modulated to vary which proteins are ultimately present in the cell, dictating its behaviour. 


\section{Sources of Inspiration}

Transcription normally begins when RNA polymerase binds to a promoter sequence on the DNA. A promoter sequence is, in simplified terms, a start marker for a particular section of genetic code. RNA polymerase will move along the DNA strand making mRNA until it encounters an end marker. Which pieces of DNA are being transcribed at any moment is controlled by regulator proteins. These proteins bind to regulatory sites on the DNA near promoter sequences, effectively turning them on or off. For example, the unicellular E.Coli bacteria prefer to feed on glucose, but can also feed on lactose if necessary [56]. Different sets of proteins are required for metabolising each of these food sources. Glucose and lactose will bind to different regulatory proteins which, in turn, will activate or deactivate the promoter sequences for manufacturing the appropriate metabolising proteins. Similarly, cells can respond directly to a variety of changes to their environment through the activation and deactivation of certain genes. Generally, the set of proteins present in a cell at any one time dictate its specialisation.

The process of building proteins to perform functions is akin to building automated tools before executing a task. Thus, since biological cells can build a large variety of tools, they can differentiate to perform a large variety of tasks.

\subsubsection{Decentralised Architecture}

Now that the various forms of redundancy that are made possible through cellular cooperation are understood, it is important to examine how that cooperation is achieved.

Complex multicellular organisms have brains which act as central task managers to coordinate system-wide behaviour. However, simple multicellular organisms, like sea sponges, do not have the equivalent of a brain [57]. In these organisms, all coordination and arbitration of tasks is handled through cellular peer-to-peer communication. Even in complex organisms with fully developed brains, a large amount of coordination is handled through inter-cellular communication. At the most primitive level, a group of cells may coordinate their movements to ensure the effective mobility of the group. Multicellular organisms utilise several methods of inter-cellular communication to coordinate activity [41].

All inter-cellular communication techniques make use of signalling molecules [58]. A cell wishing to send a message to another cell initially synthesises signalling molecules. These molecules are then transported to the recipient cell. The recipient cell has various receptors for different signalling molecules, depending on its current state of differentiation. The successful reception of a signalling molecule can trigger changes in its gene expression. Different cells may interpret the same signal molecules in different ways, depending on their 
own receptors. Inter-cellular communication methods are classified according to the means and distance over which the signal molecules are transported between cells. Methods include: Endocrine signalling, which works over long distances, Paracrine signalling, which operates between cells in close proximity, Juxtacrine signalling, which operates between touching cells and Neuronal signalling, which is very fast and point-to-point.

Endocrine signalling operates over long distances and is akin to a public broadcast. To utilise endocrine signalling, cells release their signalling molecules into the organism's bloodstream (or equivalent e.g. sap, in plants). This allows the signal molecules to spread throughout the organism. Signal molecules used for endocrine signalling are called hormones. A cell sending an endocrine message is not targeting a particular recipient. Instead, the interpretation of the message is dependent on each individual cell's receptors. For example, the hormone adrenalin is interpreted by almost all human cells. It causes the cells in the heart to increase contraction rate, blood vessels to constrict and smooth muscles cells to relax [59].

Unlike endocrine signalling, paracrine signalling molecules only diffuse over relatively short distances. Cells sending paracrine messages can only affect cells in their close vicinity. Paracrine signalling molecules are released directly by cells into the space between cells, known as the extracellular environment. The signalling molecules are short lived and diffuse slowly, causing cells closer to the sender to experience higher concentrations of the signal molecule. An example of paracrine messaging occurs during wound healing [58]. Molecules called cytokines are released into the wound area causing skin cells to express more adhesion molecules. These, in turn, help the skin to rebind.

Autocrine signaling is a special case of paracrine signalling where a cell is stimulated by its own messenger molecules. The messenger molecules are released into the extracellular environment, only to be re-detected by the same cell. In conjunction with paracrine signalling, this behaviour can be utilised by groups of cells to judge their own population densities [60].

Juxtacrine signalling requires direct contact between communicating cells. Instead of releasing messenger molecules into the extracellular environment, a cell may attach the messenger molecules to the external surface of its cell membrane. Neighbouring cells can only receive these chemical messages if they are in direct contact with the sender. Alternatively, two cells in direct contact may form a gap junction, which is a tunnel through the cell membranes of the two cells. In this way, the cells can exchange messenger molecules without releasing them into the extracellular environment. Juxtacrine signalling occurs amongst heart cells to ensure that their contractions are synchronised. 


\section{Sources of Inspiration}

A final form of inter-cellular communication is neuronal signalling. Like gap junctions, neuronal communication is point-to-point. The difference is that neuronal communication can occur over long distances thanks to special cells called neurons. Neurons have a long fiber, called an axon, which can span the distance between two distant cells. Chemical signals are converted to and from electric signals within the neuron. Neuronal communication is very fast because it uses electrical signals, instead of molecules, to transfer information. A neuronal message can be blocked if there is damage to the neuron.

Neurons form the core of an organism's nervous system. Nervous systems vary greatly in complexity between different organisms [57]. The simplest multicellular organisms, like sea sponges, have no nervous systems. In intermediately complex organisms, such as jellyfish, the neurons form a diffuse network of isolated cells called a nerve net. Finally, complex organisms have their neurons concentrated in a brain and spinal cord, with bundles of axons (called nerves), radiating to all parts of the body. In this configuration, coordination of bodily functions is largely centralised. Nervous systems allow faster and more directed communication between cells, allowing better coordination.

The vast majority of satellites have a system of coordination between subsystems analogous to a complex nervous system. A central OBC (brain) coordinates all functionality by sending messages to targeted subsystems along specialised connections (nerves). Typically, all communication between subsystems must pass through the central OBC. Similarly, in humans, the process of removing a hand from a hot surface requires a message to be sent from sensors in the hand to the brain (or at least the spinal cord) and back to the appropriate muscles. While a complex nervous system allows for better coordination amongst cells, it also presents serious vulnerabilities. In an organism, damage to the brain or certain critical nerves may prove fatal. These are potential single points of failure. Similarly, a satellite is likely to fail if it sustains damage to the OBC or critical links between the OBC and subsystems. Thus, in a system where reliability is more important than high performance, a structure and communication scheme similar to that found in simple multicellular orgasm would be effective. That is, a decentralised, distributed architecture with a peer-to-peer communication scheme akin to the endocrine system.

\subsubsection{Programmed Cell Death}

An important consideration when dealing with a group of coordinating cells is how to deal with uncooperative cells. 
In biology, cells can die in one of two ways, necrosis or apoptosis. If a cell sustains major injury from an external force, or is cut off from the blood supply, it undergoes necrosis. Necrosis is unintentional death, and the process can cause inflammation and damage to neighbouring cells. In contrast, apoptosis is cell suicide. Apoptosis is a controlled, natural and necessary process in multicellular organisms [61]. A cell may voluntarily commit suicide if it detects that it has been infected by a virus or has suffered genetic damage. Alternatively, the reception (or lack of reception) of certain intercellular messages may also trigger aptosis, if received in sufficient quantities.

Inappropriate apoptosis may lead to a variety of diseases. For example, cancer is characterised by uncontrolled cell population growth and Alzheimer's is thought to be caused by ex-cessive apoptosis [61]. Similarly, cells with genetic mutations that fail to undergo apoptosis and continue to divide can damage the health of the organism.

The importance of aptosis in multicellular organisms suggests that it should exist in some form in an artificial multicellular system, too. At a minimum, this requires an artificial cell to be able to detect anomalies in its own behaviour, and to have an isolated state which it can voluntarily enter.

\subsubsection{A Multicellular Satellite}

Based on the lessons learned regarding how biological life achieves its robustness, the following outline of an artificial multicellular satellite is proposed:

Instead of the avionics being based on a set of unique, discrete subsystems, a multicellular satellite's avionics are based on a set of artificial cells. Every cell is electrically and mechanically identical when it comes off the assembly line. Every cell contains an identical set of 'DNA', in the form of non-volatile instructions, which specify how to perform every potential task of its host system. Once a set of cells have been assembled into a complete system, the artificial cells communicate with one another over a peer-to-peer network and coordinate their 'differentiation'. Through some mechanism which reads the DNA, every cell reconfigures to take on a specific task within the system. Once the system is configured and running, the cells constantly monitor their own and their peers' health. In the event of a cell failure, the cell can be isolated and the remaining healthy cells coordinate to take over from the failed cell. Through this process, the satellite may degrade in capabilities or performance, but the process ensures that the most critical tasks remain executed. 
Such an approach to low-cost satellite development will only be advantageous if the following conditions can be met:

1. The resources required to develop a generic cell must be lower than the resources required to develop the set of unique subsystems that are to be replaced by cells.

2. Cells must be sufficiently capable in their reconfiguration process so as not to impose significantly more overheads than the dedicated subsystems they are replacing. In other words, if a cell is to replace a subsystem, minimal additional external circuitry must be required.

3. The process of differentiation must not invalidate electrical or environmental testing that has been performed on a cell.

By fulfilling these conditions, a multicellular architecture could demonstrate the following benefits:

1. Graceful degradation could replace 'instant death' on missions with minimal resources for backup redundancy.

2. Cell mass production could lead to reduced manufacturing costs.

3. Uniform, automated test procedures could save time and more accurately identify manufacturing faults.

4. Simplified integration, thanks to identical electrical and mechanical interfaces, could lead to fewer integration mistakes and reduced development time.

Despite the promise of such an architecture, there are potential disadvantages, too. The reconfigurability of the cells will likely lead to overheads in mass, volume and power consumption. In addition, the firmware development will likely be complex and the distributed nature of the architecture has the potential for instability. The only accurate way to determine the costbenefit ratio of the proposed, conceptual system is through the design and implementation of a prototype multicellular system.

To this end, the remainder of this thesis describes the development of the Satellite Stem Cell Architecture, and the investigation of its practical feasibility through analytical reliability analysis, simulation, and the development and benchmarking of hardware prototypes. 


\subsection{Bridging Research Fields}

While the architecture of multicellular life serves as the main inspiration behind this work, several other research fields provide contributions, too. These include bio-inspired processors, agent computing and modular spacecraft concepts. In the remainder of this chapter, important works from each of these fields are discussed, and the expected contributions of the Satellite Stem Cell Architecture are highlighted.

\subsubsection{Bio-Inspired Processors}

Like the work described in this thesis, many other projects have been inspired by the architecture of biological life. Bio-inspired processors are man-made computing systems which mimic, or take inspiration from, various biological processes. Bio-inspired processors found in literature vary significantly in capability, with some projects aiming for increased fault tolerance, while others seek to accurately model biological processes. Many are implemented within a single FPGA, where structures are more easily duplicated and reconfigured than in discrete hardware. In this section, three prominent examples of bio-inspired processors are described, including Embryonics, POEtic, and eDNA.

\subsubsection{Embryonics}

The Embryonics project is considered a classic amongst researchers of bio-inspired processors. Its stated objective is: 'the conception of very large scale integrated circuits endowed with properties usually associated with the living world: self-repair (cicatrisation) and selfreplication' [62]. The Embryonics design is based on a two-dimensional grid of cells, each of which has a three-layered hierarchical structure (Figure 3.3).

The bottom layer of each cell contains a basic computational element called a MUXTREE. It is composed of a 2-to-1 multiplexer and a D-type flipflop, together with interconnection switches allowing the inputs and outputs of the MUX and flip-flop to be connected to neighbouring cells. Each MUXTREE is configured using a 20-bit word, or gene. Each bit in the gene acts on a control line of the MUX, flip-flop, or bus switches. Complex logic functions, described using ordered binary decision diagrams, can be built up by interconnecting a set of MUXTREEs. The authors of Embryonics describe cells configured with different genes as being in different states of differentiation. 


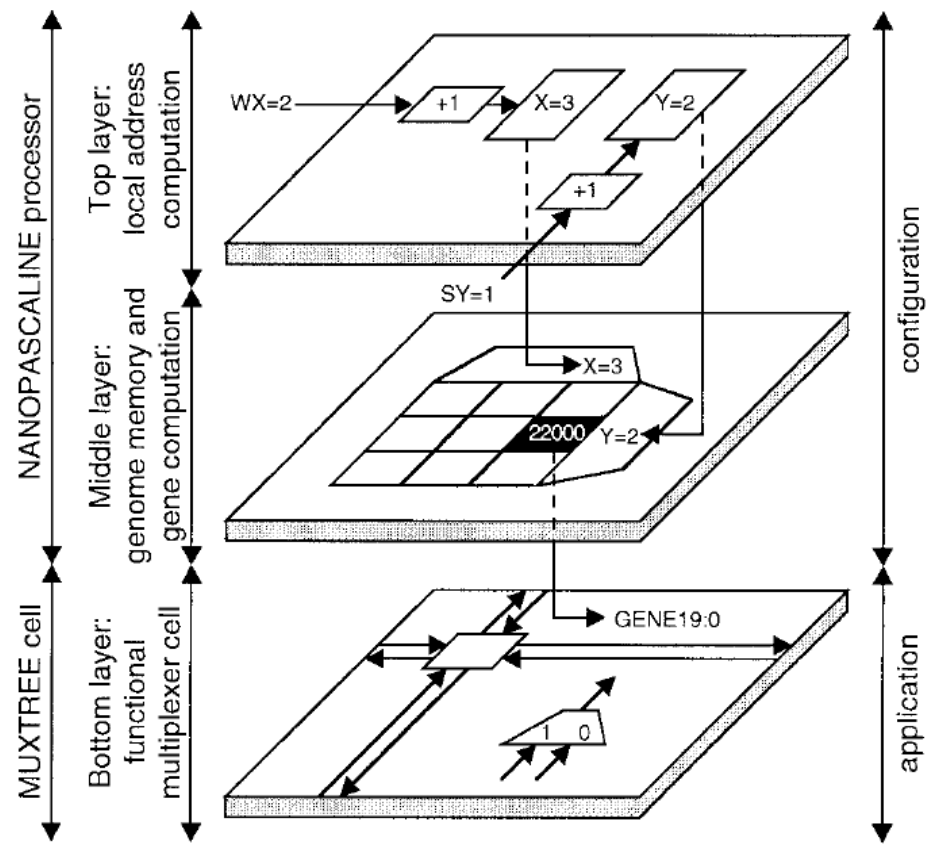

Figure 3.3: The three-level hierarchy of the Embryonics architecture. The top two layers are only active during the initial differentiation of cells, while the bottom layer is always active [62].

The second layer of an Embryonics cell is composed of random access memory, which stores the cell's 'genome'. The genome contains all the individual genes required to build the desired complex logic function. Despite every cell containing the entire genome, each cell's MUXTREE will only be configured using a single gene of the genome.

The process of selecting which gene to load into the MUXTREE is handled by the top layer of the artificial cell. The authors compare this process to that performed by ribosomes in biological cells. In an Embryonics cell, a binary decision machine, or interpreter, executes a microprogram to determine with which gene to configure the MUXTREE, based on the cell's $\mathrm{X}$ and $\mathrm{Y}$ coordinates within the grid. The genome memory and interpreter layers are only active during the initial configuration of the cell. Thereafter, only the MUXTREE level of the cell is active.

In addition to configuring its cell MUXTREE, the microprogram also copies itself and the genome to empty neighbouring cells, effecting a form of self-replication. Thus, only a single cell within the grid initially needs to be manually programmed with the genome in order for the complete genome to eventually be expressed by a whole set of cells across the grid.

The Embryonics authors also describe a form of self-repair, where faulty cells are isolated before the self-replication process produces new cells to take over from the faulty ones. How- 
ever, the original research paper describes it as a process which relies on human intervention to identify and signal the presence of a faulty cell.

Two partial implementations of the Embryonics architecture were developed to demonstrate the feasibility of MUXTREE-based logic functions. One implementation contains 240 cells based on Actel 1020A programmable circuits, while the other is based on a custom developed ASIC of 64 cells. However, each of these cells contained only the MUXTREE level.

Follow on research includes a reliability analysis of the Embryonics architecture [63] and work on combining Embryonics with the research field of Immunotronics [64].

\subsubsection{POEtic}

The POEtic project highlights the heterogeneous nature of bio-inspired hardware to date, noting that the lack of a universal development platform has prevented the emergence of a methodology in the design of bio-inspired computing systems. To this end, the aim of the POEtic project is the: '...development of a flexible computational substrate inspired by the evolutionary, developmental and learning phases in biological systems' [65]. The POEtic project does not aim to implement a specific bio-inspired architecture. Instead, it aims to provide a flexible platform, composed of a grid of hardware 'molecules', which can be configured into cellular structures to study the potential hardware implementations of bio-inspired algorithms and systems.

According to the members of the POEtic project, bio-inspired systems principally find their inspiration in three biological models: phylogenesis, ontogenesis and epigenesis. Phylogenesis is concerned with the evolution of a species over time, and has inspired work such as genetic algorithms, evolutionary programming, and genetic programming. The ontogenetic model describes the development of an individual based on its genetic code. Cellular division and differentiation are key processes in the ontogenetic model. Due to the difficulties involved in developing self-replicating machines, study in this area was limited until the invention of FPGAs. Now, projects such as Embryonics are attempting to mimic ontogenetic processes to imbue integrated circuitry with features such as self-replication and self-repair. Finally, the epigenic model describes how interactions with the environment can shape the development of an organism. Research into epigenetic mechanisms has led to the research fields of artificial intelligence and artificial neural networks. 


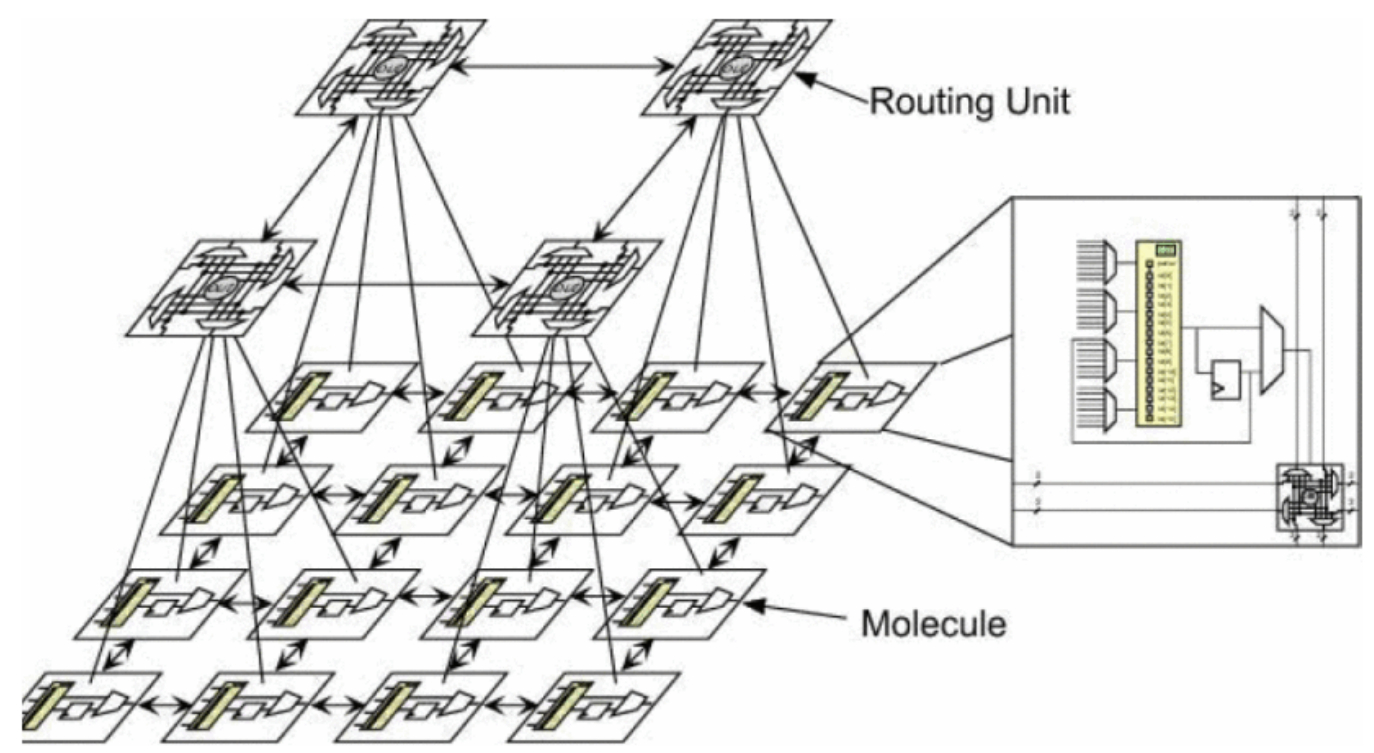

Figure 3.4: The organic subsystem of the POEtic architecture. It is composed of a grid of reconfigurable 'molecules' and a grid of communication switchboxes. The molecules can be combined into a variety of experimental artificial cells [65].

In order to facilitate research based on these three models, the POEtic development platform is composed of three parts: the organic subsystem, environmental subsystem, and system interface.

The organic subsystem, shown in Figure 3.4, is composed of a grid of elementary units called molecules. Each molecule is composed of a four-input lookup table, a register and a switchbox. A molecule can be configured to perform a variety of simple logic functions, including being a shift register or multiplexer. These functions have been selected as useful building blocks for implementing artificial cells. For example, a shift register can be used to store a simple binary-encoded genome. By combining molecules and configuring their interconnections through the switchboxes, different implementations of digital cells can be realised. In addition to the grid of molecules, the organic subsystem also contains a grid of routing units which can be used to implement dynamic routing mechanisms between constructed cells.

The purpose of the environment subsystem is to allow interactions between between the environment and the organic subsystem. Such interactions can come from physical sensors and actuators interfaced to the environment subsystem, or from simulated environments for studying, for example, evolutionary algorithms. Epigenetic processes will thus largely be implemented in the environmental subsystem. The environmental subsystem is composed of a 
custom microprocessor and various peripherals connected by an Advanced High-Performance Bus (AHB). One of the peripherals is a random number generator, which has specifically been included to aid in implementing the randomness of evolutionary processes.

Finally, the system interface performs an integration function by mapping the configuration bits of each molecule in the organic subsystem to the memory space of the environment subsystem. Thus, researchers are able to implement processes which dynamically reconfigure the molecular fabric. In addition, the system interface facilitates scaling of the development environment by providing a communication link to other system interfaces. The system interfaces can transparently map several organic subsystems together into a single larger one.

The POEtic system has been implemented as a custom ASIC [66]. It contains twelve molecules, together with the environmental subsystem and system interface. In addition, various development tools have been created to facilitate the use of the chip, including a graphical simulation tool.

\subsubsection{3 eDNA}

The eDNA project aims to create a fault tolerant, self-healing processor IC [67]. Its architecture, shown in Figure 3.5, is based on an array of homogeneous processing units, called eCells, which communicate with one another over a two-dimensional mesh network. Together, the eCells work to execute an eDNA program, a copy of which is stored in each eCell. Each eCell executes only a specific part of the eDNA program, mimicking the biological process of gene expression.

Each eCell is composed of a small, 8-bit MicroBlaze MCU, a 32-bit arithmetic logic unit (ALU), and a block of memory for storing the eDNA program. eDNA programs are written in a bespoke programming language which allows the program to be compiled into a set of discrete ALU operations, or genes. The complete eDNA program is loaded into each eCell, and each of its genes are executed in a distributed fashion by the ALU's of the eCells. The MCU is responsible for executing a program known as the ribosomal DNA, which performs the self-organising and self-healing functions of the eDNA architecture. Based on its eCell's coordinates within the grid, the MCU locates a specific gene of the eDNA program and uses it to configure the ALU. Additionally, the MCU configures the appropriate interconnections to the ALUs of other eCells using the mesh network. 


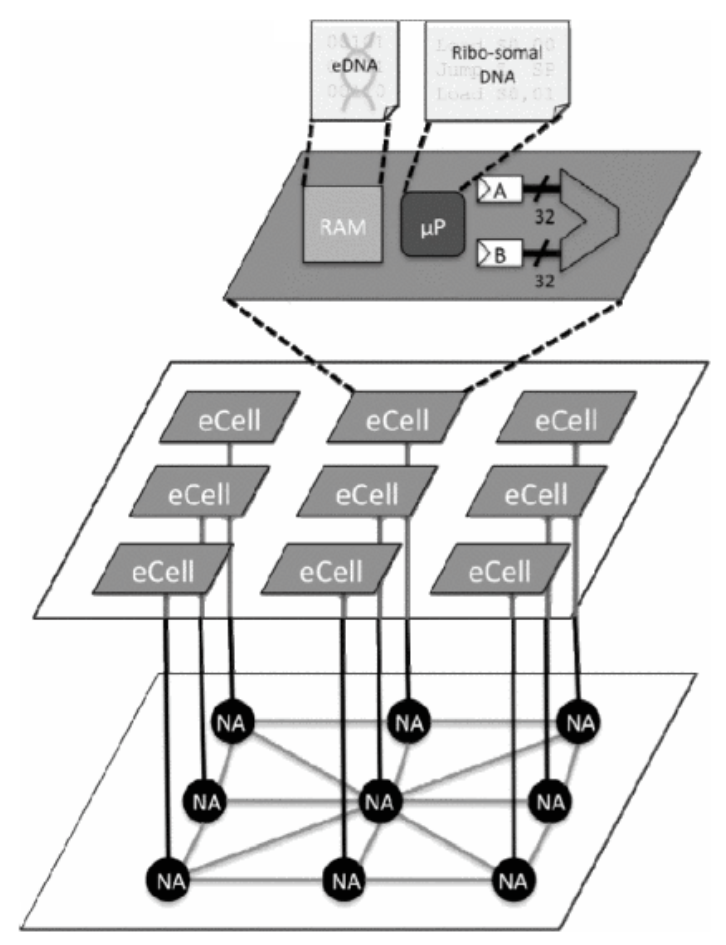

Figure 3.5: The eDNA architecture is composed of a grid of eCells and a mesh communication network facilitated by Network Adapters (NA). Each eCell contains an MCU, ALU and DNA memory [67]. 
The eDNA system is capable of recovering from eCell failures through a self-repair mechanism, provided spare eCells are present. Once a failure is detected, a spare eCell is invoked to begin executing the gene of the lost eCell. Since every eCell already contains the complete eDNA program, no reprogramming is required. However, as part of the fault repair mechanism, the MCU will attempt to transfer run-time state variables across to the replacement eCell.

A 3 x 3 eCell architecture has been implemented in a Xilinx Virtex 5 FPGA [68]. As part of a practical demonstration, the eCell system was integrated into an experimental science instrument called a Fourier Transform Spectrometer. The small scale of the 9-eCell implementation limited the demonstration to two simple eDNA programs. One generated a voltage ramp, while the other performed the averaging of voltage readings from the instrument. The results showed that the eDNA implementation is 6 - 90 times slower and uses approximately 5 times more FPGA resources than a traditional HDL implementations. However, it also demonstrated that the self-healing time is a fraction of the execution time of an application. In addition, the eCell architecture's intended migration to an ASIC implementation is expected to increase its performance and decrease overheads.

\subsubsection{Multi-Agent Systems}

The concept of agent computing emerged in the 1990s as a new, high-level programming philosophy [69]. Software agents are programs which can make autonomous decisions within their environment to meet design objectives. Similar to the cells in a multicellular organism, agents have life cycles, pursue goals and may have a level of mobility. Thus, agent technology has the potential to provide a natural solution for facilitating communication and cooperation between the artificial cells of a multicellular system.

As the name suggests, a multi-agent system consists of platform on which multiple agents can coexist and cooperate. Agent platforms can be categorised according to their target hardware platforms, their real-time capabilities, and their compliance to a standard. Target hardware platforms range from desktop computers with gigabytes of RAM and multigigahertz processors, to physically-tiny, embedded platforms based on MCUs clocked at a few megahertz and with only a few kilobytes of RAM. An agent platform's real-time capabilities are determined by its agent execution environment (AEE), which may be based on an interpreter, virtual machine, or native hardware. Finally, an agent platform aiming to form part of a larger heterogeneous agent environment may choose to adhere to one of several agent standards. For example, the Knowledge Query and Manipulation Language 
(KQML) [70] and the Common Object Request Broker Architecture (CORBA) [71] can be implemented as standards for inter-agent communication. Amongst agent standards, those of the Foundation for Intelligent Physical Agents (FIPA), covering many aspects of agent communication, behaviour and management, are the most modern and have proven to be the most popular [72].

\subsubsection{Traditional Agent Platforms}

The most common agent platforms are those designed for standard personal computer hardware. Typical applications for such a platform include multi-agent-based simulations applied to a variety of research fields, including economics, social sciences, biology and urban planning. As these applications do not have real-time requirements, agents are commonly coded in Java and execute within Java Virtual Machines (JVMs). A good survey of 24 such platforms, including the popular, FIPA-compliant examples JADE [73] and FIPAOS [74], can be found in [72]. While these platforms are powerful, multifunctional, and allow code mobility, their agent execution timing is non-deterministic due to the underlying JVMs garbage collection routines, class initialisation, and just-in-time compilation [75]. Additionally, their large memory footprints make them undesirable for use in the resourcerestricted, embedded environment of a satellite.

\subsubsection{Embedded Agent Platforms}

Several agent platforms with reduced memory footprints have been developed for deployment on smartphones. Examples include JADE-LEAP [76], u-FIPA [77] and AgentLight[78]. These platforms typically make use of the JAVA ME Embedded or Android runtime environments as the basis for their agent execution environments. The Java ME Embedded runtime environment is available for many platforms, including ARM Cortex M3 MCUs and requires as little as $128 \mathrm{kB}$ of RAM and $1 \mathrm{MB}$ of ROM [79]. Experiments with AgentLight have demonstrated the creation of ten agents using only $83 \mathrm{kB}$ of memory.

The FIPA-compatible Mobile-C agent platform was specifically developed to have a small memory footprint, and differs from the majority of agent platforms by using an embedded $\mathrm{C} / \mathrm{C}++$ interpreter as its AEE [69]. The embedded interpreter claims to be smaller than the Java ME Embedded runtime environment and allows agents to be written in the $\mathrm{C}$ programming language. Writing agents in $\mathrm{C}$ simplifies the process of interfacing to low-level hardware, which is a common requirement in embedded applications. 
Even smaller agent platforms have been developed by the distributed sensor node community. Agilla, for example, requires only $57 \mathrm{kB}$ of ROM and $3.3 \mathrm{kB}$ of RAM when deployed on an ATmega128L MCU [80]. Agilla is not FIPA compatible and its agents are programmed in a custom language and execute in a bespoke virtual machine.

While agent platforms based on the Java ME Embedded runtime environment, Mobile-C, or Agilla would fit within the processing power and memory constraints of a satellite embedded system, the non-deterministic nature of their AEEs make them poorly suited for real-time tasks such as attitude determination and control.

A solution to this problem is to replace the JVM with a hardware Java processor. Bridges et. al. attempted to get the JADE-LEAP mobile agent platform operating on such a processor, with the aim of developing a real-time agent platform for satellites [81]. The Java Optimised Processor (JOP) was chosen and implemented in a Xilinx Spartan-3 FPGA. While the feasibility of the concept was proven, a usable platform could not be developed.

The Secure Hardware Agent Platform (SHAP) is an alternative Java hardware processor which was designed by Zabel et. al. specifically to enable the creation of a real-time agent platform [82]. It has been demonstrated running the Connected Limited Device Configuration (CLDC) API, which is a subset of the Java ME runtime environment. However, it appears that no agent environment based on this processor has been developed yet.

\subsubsection{Agents in Space}

Despite the challenges of getting an agent platform executing on the constrained hardware of a satellite, at least one agent has seen in-orbit operation. In 1999, NASA briefly handed control of its Deep Space 1 probe to an experimental on-board agent [83]. The agent successfully developed and executed plans for dealing with a variety of simulated events. However, it was designed as high-level software and did not perform real-time tasks. More recently, the ObjectAgent environment was developed by Princeton Satellite Systems for its Techsat 21 mission [84]. However, this agent platform is designed to run on a powerful PowerPC 750 processor, is not FIPA compliant, and was never completed as TechSat-21 was cancelled. 

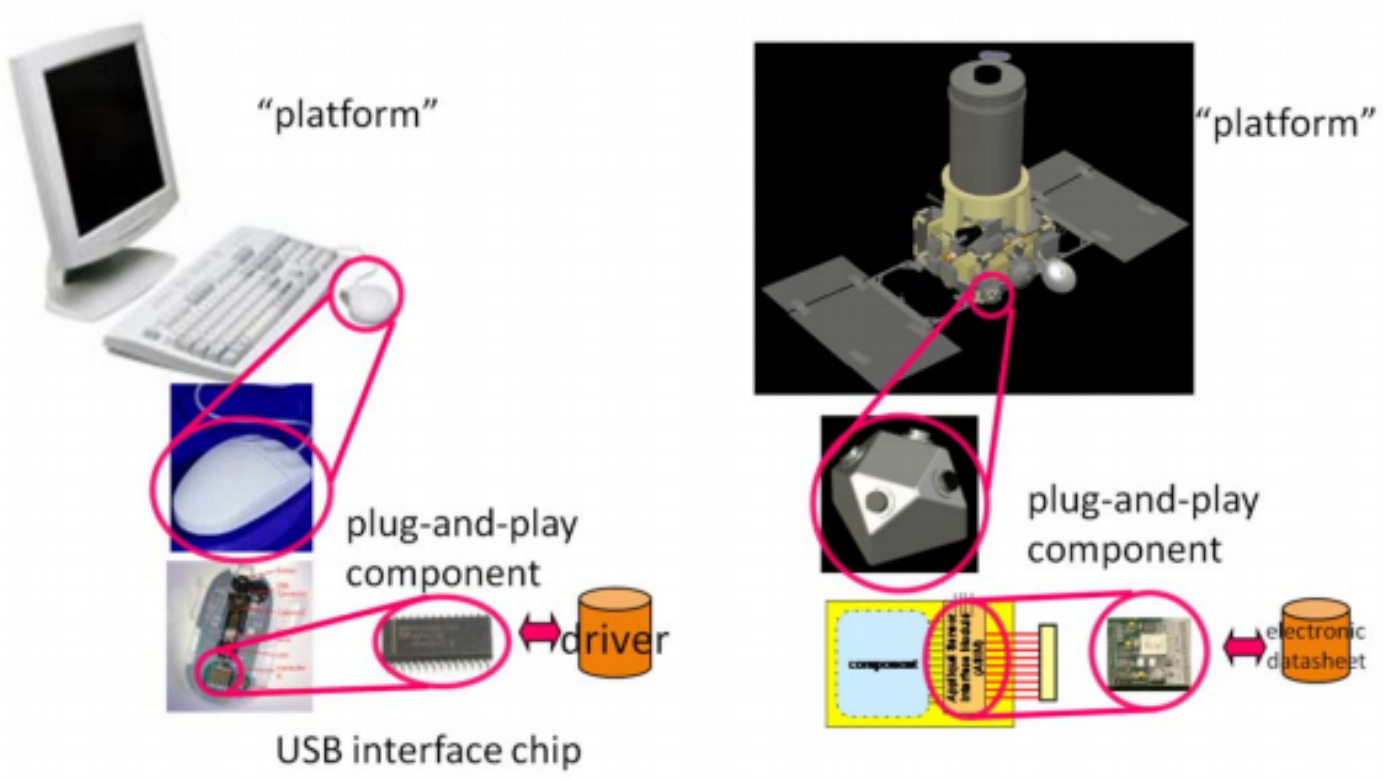

Figure 3.6: The Space Plug-and Play architecture envisions satellite subsystems and peripherals interfacing as seamlessly as the peripherals of modern desktop computers [85].

\subsubsection{Modular Satellite Concepts}

Not many attempts have been made at designing a generic satellite building block. While many COTs subsystems are available, they are usually built for purpose and are designed to be reused across missions, not within the same satellite. Additionally, while some electrical interfaces have become standardised, such as SpaceWire, mechanical, electrical supply, and TTC interfaces are rarely standardised or compatible between manufacturers.

The two most interesting and relevant modular satellite concepts are the Space Plug-and-Play architecture, and DARPA's Phoenix Project.

\subsubsection{Space Plug-and-Play}

The Space Plug-and-Play Avionics (SPA) architecture [86] is inspired by consumer computer plug-and-play interfaces, like USB, which have become very successful (Figure 3.6). It has been under development by the US Air Force for more than a decade. Its goals include:

- Build systems dramatically faster (satellite in a week).

- Promote hardware and software reuse. 


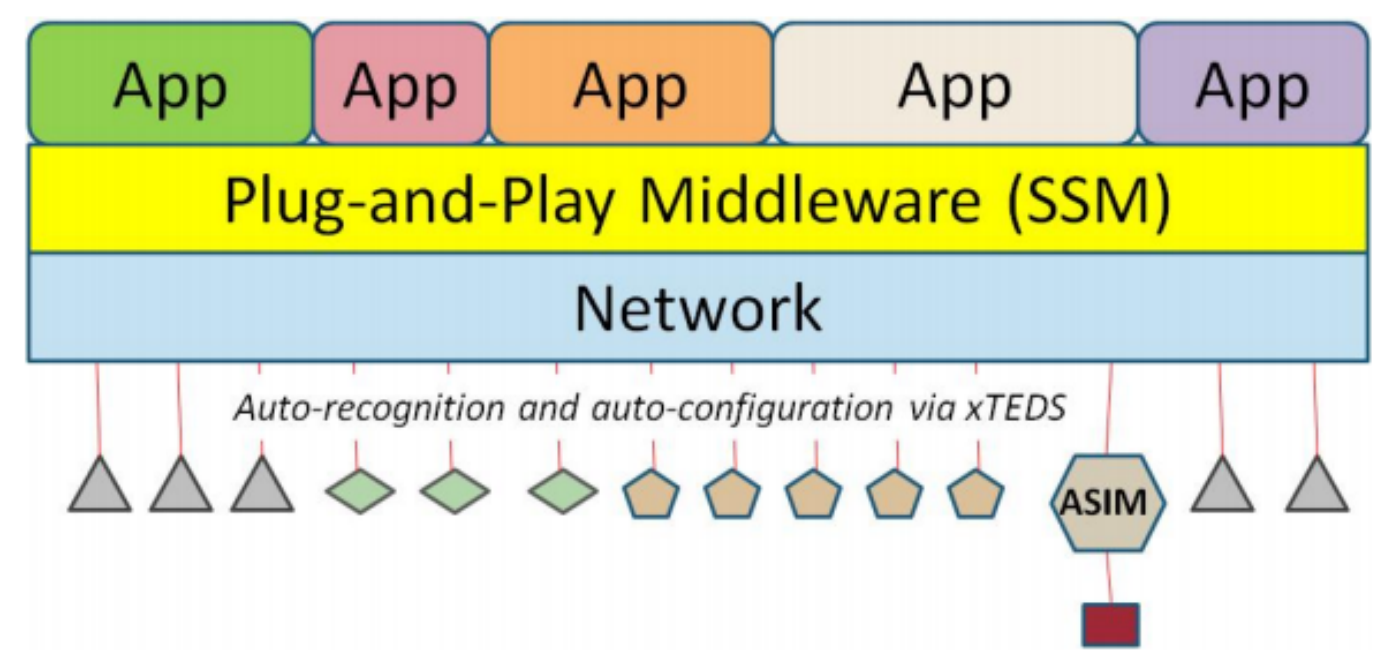

Figure 3.7: The SPA network architecture [85].

- Aim for an 'open architecture' by avoiding obscure and proprietary data handling protocols.

- Make the addition of redundancy simple and flexible

The SPA architecture is composed of both hardware and software elements. The hardware elements include a set of defined, electro-mechanical, point-to-point interfaces, a set of routers, and an Application Sensor Interface Module (ASIM), which allows legacy devices to interoperate with the rest of the system. Together, they allow a set of SPA compatible nodes and peripherals to be networked into a complete system. The software elements consist of an electronic datasheet embedded in each node (or ASIM), and a set of middleware which manages the SPA network. The electronic datasheet is called the eXtensible Transducer Electronic Datasheet (xTEDS) and allows a node plugged into the SPA network to identify itself and its capabilities. As shown in Figure 3.7, the middleware is called the SPA Service Manager (SSM), which runs on top of Linux and facilitates the discovery, registration, and transfer of data between nodes.

The SPA architecture is not designed to be completely distributed. Instead, the SSM middleware is hosted on a central node. Nodes performing different functions can be electrically and mechanically different, as long as they abide by the interfacing standards. In collaboration with the Swedish company AAC Microtech, a number of different SPA nodes have been developed. The smallest node, called the nanoRTU (RTU - remote transceiver unit), is based on an FPGA with a softcore PIC 16F84 processor. The nanoRTU is essentially an ASIM, designed to allow legacy devices to access the SPA network. It is an intrinsically digital 


\section{Sources of Inspiration}

device and cannot interface directly to analogue sensors and actuators. However, a generic analogue interface add-on has been created for it. Two larger devices, the Micro-RTU, which consumes $1.5 \mathrm{~W}$, and full RTU, which is still in development, are designed for interfacing to more complex payloads. They are also capable of hosting the SSM middleware.

Work on the SPA architecture over the last decade has resulted in at least ten standards documents being approved by the AIAA [85]. Furthermore, the TechEdSat 1U CubeSat, based on the SPA architecture, was launched in 2012 and proved to be very successful. It contained a Micro-RTU as its central OBC, which hosted the SSM middleware, and a number of nano-RTUs which served as interfaces to other subsystems and payloads [87].

\subsubsection{DARPA Phoenix Project}

The DARPA Phoenix program highlights the fact that the construction and make-up, or morphology, of satellites has barely changed over the past fifty years. Satellites are typically constructed out of subsystems which are sized to achieve certain performance requirements. In many cases, the process of sizing a subsystem is based on certain rules of thumb, which have remained relatively unchanged due to the inherent risk-adversity of the space industry. For example, both geosynchronous satellites weighing several tonnes and CubeSats, weighing less than $3 \mathrm{~kg}$, use essentially the same reaction wheel mechanism for attitude control. The wheels only vary in size. Thus, an almost linear cost-mass-performance function has emerged. Lately, focus has been placed on lowering the mass of satellites to reduce cost. However, these efforts have largely been met with an equivalent reduction in performance, as is commonly seen amongst CubeSats. To this end, the DARPA Phoenix program aims to: "re-define the cost-mass-performance function, by challenging the existing satellite morphology and demonstrate the concept of "cellularity" [88].

In this case, the concept of 'cellularity' was also loosely inspired by multicellular life. The Phoenix 'cells' are proposed as CubeSat-scale 'satlets' which will be assembled in space into larger satellites. Assembly is envisioned to be performed by a larger constructor satellite, and not autonomously by the satlets themselves.

As part of the Phoenix project, a study was undertaken to determine the optimum number of unique satlet types, largely based on economic factors. This optimum was determined to be four, namely: central, actuator, structural and payload satlets [89]. The central satlet is essentially a complete nanosatellite, featuring data processing, attitude sensing, and ground communication capabilities. The other satlets are less intelligent and are unable to function independently. All satlets feature mounting points and power and communication ports, 


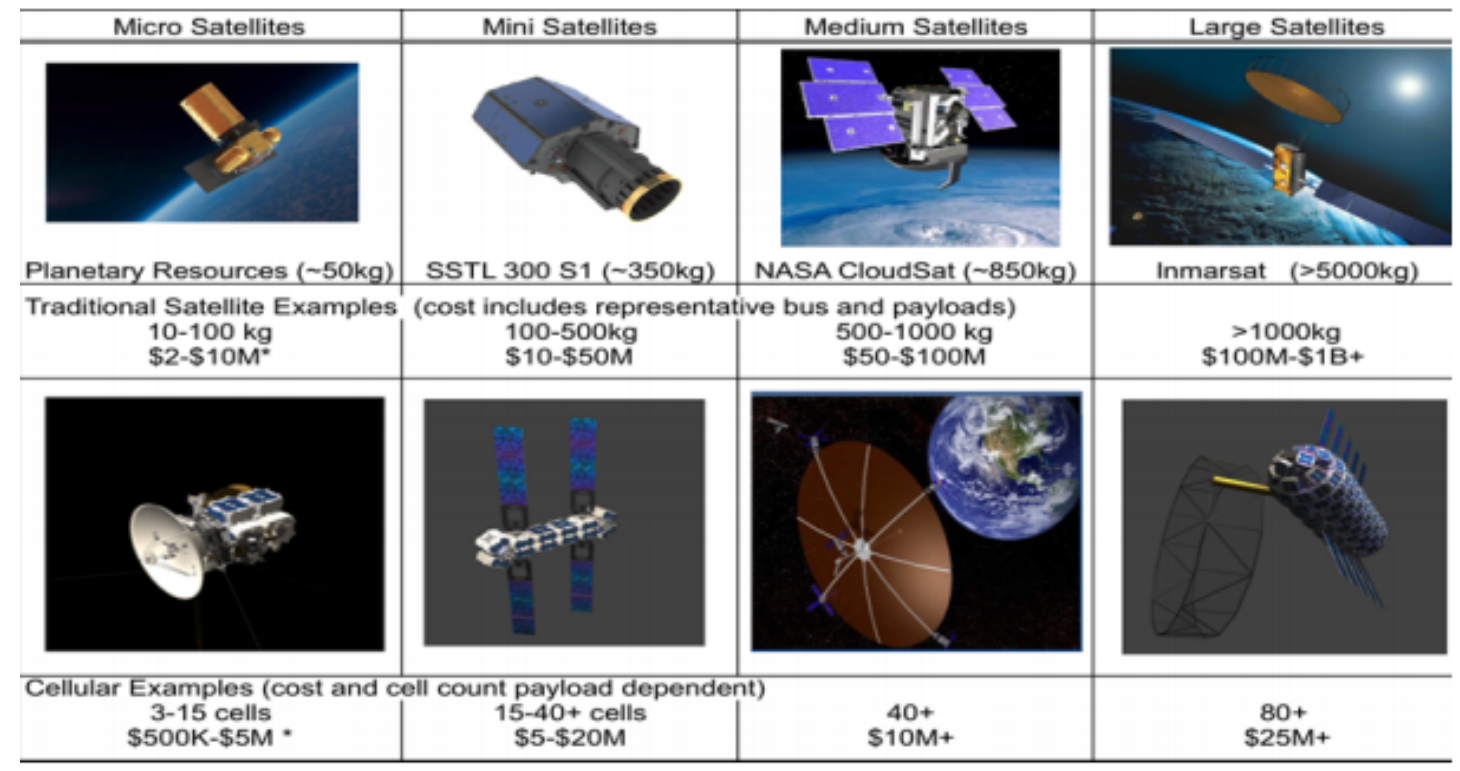

Figure 3.8: Comparison between traditional satellites of varying sizes, and their 'satletised' counterparts [90].

allowing them to be aggregated into a single larger entity. As shown in Figure 3.8, it is proposed that satellites of all sizes, from nano $(<10 \mathrm{~kg})$ to large geosyncronous satellites could be assembled out of a combination of these four satlet types. Different mission requirements will be served by different combinations of satlets. For example, a highly agile mission will include more actuator satlets, while a telecommunication satlet might contain a large number of structural satlets in the structure of its dish antennas.

The Phoenix program aims to disrupt the cost-mass-performance function in two ways. Firstly, by building all satellites out of the same four satlet types, the Phoenix program hopes to make use of mass production techniques. They estimate, based on current satellite demand, that there could be a market for 1000 to 16000 satlets per year, which is two orders of magnitude higher in production quantity than any currently produced satellite subsystem [89]. Secondly, the Phoenix program aims to reuse satlets which are still functional at the end of their mission lifetimes. For example, large dish antennas, which are difficult and expensive to launch, are specifically targeted as items which, if constructed out of satlets, could be transferred from one satellite to another at the end of one mission's lifetime.

The Phoenix project also notes that its satlet-based architecture allows designers to easily trade reliability against other system traits during development. For example, changes in the mission lifetime requirement which come during the satellite design phase could be accommodated by removing an actuator satlet and replacing it with a spare central satelet. In 


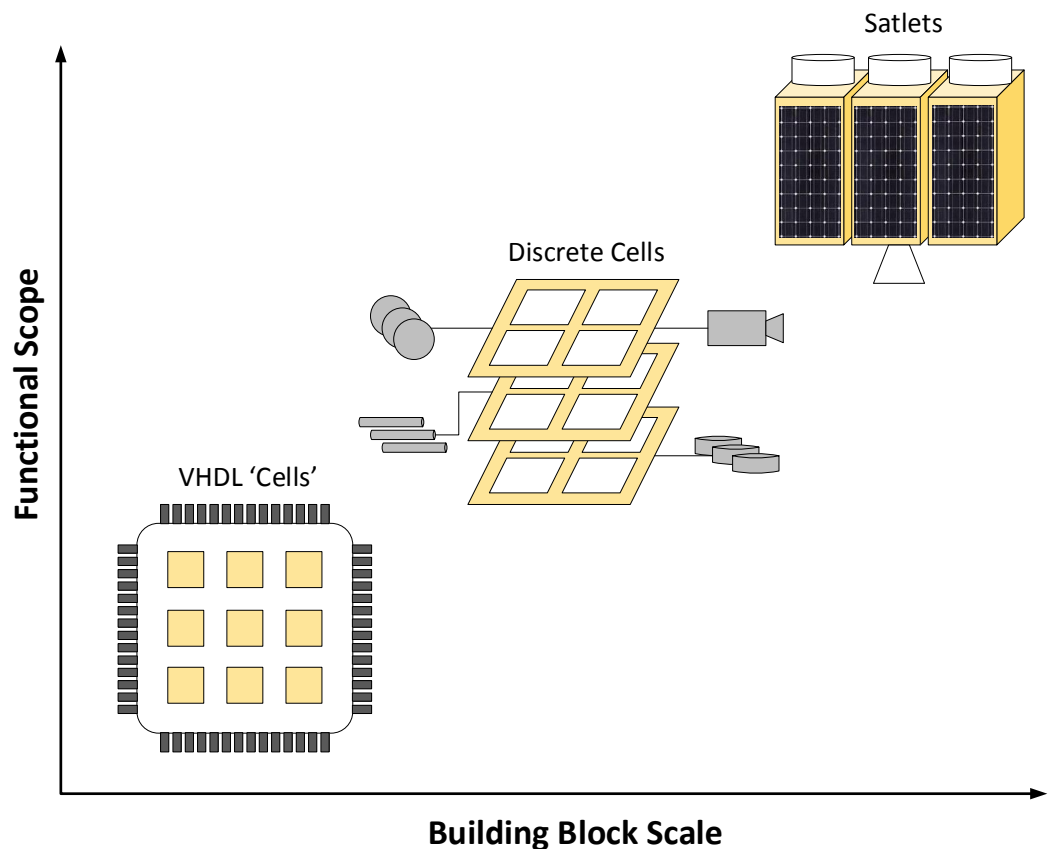

Figure 3.9: Different scales of system cellularisation based on cells of increasing functional scope.

this way, the satellite will lose some manoeuvrability performance, but will gain important redundancy. This process is simpler than the traditional process which would involve, for example, reducing the mass of the reaction wheels to free up some mass budget for the addition of critical system redundancy.

An implementation of the satlet concept exists in the form of NovaWurks HiSat platform. Each highly integrated satellite (HiSat) is a complete satellite measuring $20 \times 20 \times 10 \mathrm{~cm}$ [91]. Their structures feature locking mechanisms and electrical ports, allowing them to be assembled into larger structures. The eXCITe mission, scheduled to launch in late 2017, will carry a pre-assembled satellite composed of several satlets into orbit [90]. Its primary aims will be to test the mechanical design's ability to survive launch, and to demonstrate the satlets' ability to function independently and together.

\subsubsection{Discussion and Expected Novelties}

The proposed Satellite Stem Cell Architecture, bio-inspired processors, and modular satellite concepts discussed in this chapter demonstrate 'cellularisation' at different scales and functional scopes. This is depicted in Figure 3.9. 
At the lower end of the scale, bio-inspired processors, such as Embryonics, POEtic and eDNA, demonstrate how aspects of biological cells can be applied at the logic gate level of FPGAs and ASICs to enable features such as self-healing and functional reconfiguration. Such features would be especially useful when trying to replace expensive space-rated FPGAs with COTs ones. The 'cells' in these projects are composed of simple logic elements or elementary processors. Their greatest advantage in terms of reliability is their individual simplicity. Further advantages of cellularisation in this form include rapid prototyping (if built in a hardware description language), and large cell populations (extensive internal redundancy).

However, when aiming for a practical system with fault tolerance at system-scale, currentgeneration, logic-scale implementations have several disadvantages. Firstly, current generation implementations are based on digital circuits (which include the vast majority of FPGAs), which mean they are limited to processing applications. Additional circuitry is required to interface these bio-inspired processors into practical sensor-actuator systems, and it is impossible for them to provide fault tolerance for other aspects of the system (e.g. batteries, structure). Secondly, their limited functional scope is compounded by the fact that projects such as Embryonics, POEtic and eDNA aim for accurate bio-mimicry at the expense of performance. They require programs written in bespoke languages, bespoke compilers, and are not designed with real-time applications in mind. Therefore, they are currently limited to relatively simple lab demonstrations.

At the other end of the scale, cellularisation is applied at system scale. An example is the DARPA Phoenix Project, which proposes building a larger satellite out of multiple, smaller satelets. Theoretically, each satlet is an almost fully functional system itself. The main advantage of applying cellularisation at this level is that every aspect of the system can be made redundant. This includes processing capacity, avionics, actuators and structure. In other words, each 'cell' has a large functional scope.

However, there are several disadvantages that go along with a large functional scope. Firstly, each satlet is a complex system in itself, and therefore less reliable (especially when compared to the simplicity of a cell composed of a few logic gates). Further, as the scale of the cells increase, so the total number of cells in the system is likely to decrease. This is simply due to logistical and budgetary limitations. Finally, the complexity of the mechanism required to replace a failed cell with a redundant one increases as the functional scope of the cells increases. For example, replacing a logic-based cell within an FPGA with a spare simply involves activating a few switches to reroute signals. However, physically moving a satlet 
to repair structural damage to another area of a multicellular satellite would require a very complex (and in the case of the Phoenix Project, external) mechanism.

Thus, the Satellite Stem Cell Architecture, proposed in this thesis, aims for a novel middleground in terms of scale and functional scope.

Compared to bio-inspired processors, the Satellite Stem Cell Architecture aims to distinguish itself by extending beyond pure computational tasks. Through the use of generic I/O circuitry, it aims to allow each cell to interface directly to a variety of peripherals, allowing the implementation of complex sensor-actuator systems with minimal additional hardware. Furthermore, while most bio-inspired processors to date have been lab experiments, the Satellite Stem Cell Architecture places importance on the practical feasibility of the design. In addition to providing sufficient processing capacity to perform complex tasks such as attitude control, the Satellite Stem Cell hardware must be physically small and consume minimal power.

The repair mechanism of the Satellite Stem Cell Architecture will be facilitated by a novel set of middleware based on the concept of Agent Computing. Due to the resource-constrained and real-time nature of a small, low-cost satellite platform, the developed agent platform is envisioned to differ from traditional agent platforms in three areas. Firstly, the developed agent platform will require a very small memory footprint - on the order of tens of kilobytes. Secondly, real-time task execution is required, which excludes the use of interpreters and virtual machines. Finally, in addition to the usual services provided by an agent platform, the developed platform will be required to execute a distributed task allocation strategy.

Compared to the DARPA Phoenix project, the proposed artificial cells have a much smaller functional scope. They aim to cellularise only a system's processing elements and portion of the analogue electronics. However, unlike the Phoenix Project and Space Plug-andPlay modular spacecraft concepts, the Satellite Stem Cell architecture envisions a fully decentralised architecture, where every cell has the potential to perform the same tasks and reconfiguration is handled without external, or centrally coordinated, help.

\subsection{Summary}

This chapter presented an in-depth investigation of the techniques employed by unicellular and multicellular life to achieve robustness. Unicellular life was found to employ techniques which are well known to system developers. These include the use of specially adapted 
proteins (radiation-hardened parts), genetic redundancy (functional redundancy), and gene repair (error detection and correction schemes). Multicellular life, on the other hand, displays techniques which are not commonly employed by man-made systems. They are based on the biological processes of differentiation and inter-cellular communication.

With the aim of adapting these techniques for use in man-made systems, an outline for a novel, artificial, multicellular architecture was presented. It is based on initially-identical, reconfigurable, hardware blocks, instead of unique, discrete subsystems. The proposed architecture has the potential for enabling graceful degradation, mass production, streamlined testing, and simplified integration.

The second part of this chapter presented important works from several other research fields, including bio-inspired processors, agent computing, and modular spacecraft concepts, from which key ideas and inspiration were taken. 



\section{Chapter 4}

\section{Artificial Stem Cells}

In Chapter 3, the unique characteristics that make multicellular life robust were discussed and the outline of an artificial multicellular architecture was proposed. In this chapter, the concept is further developed through the design of a new artificial stem cell. These cells are designed to replace the avionics of traditional satellite architectures and to interface directly to a variety of sensors and actuators. Since the proposed architecture has these cells at its core, it has been named the Satellite Stem Cell Architecture.

Ideally, Satellite Stem Cells should replace all the avionics of a satellite. This concept is more feasible than it seems at first. Through the advancement of integrated circuit technology and software development environments, an increasing amount of discrete circuitry is being replaced by microcontrollers and field-programmable gate arrays (FPGAs). This shifts functionality from hardware to software, which can more easily be updated. Furthermore, functionality which cannot be performed in software is typically implemented using a small set of commonly used circuit blocks, such as high-power switches and analogue to digital converters (ADCs). Thus, a generic hardware block, or cell, containing a microcontroller and some of these common circuit blocks already contains the core of most traditional subsystems.

However, subsystems which still contain a large portion of analogue electronics, such as radio transceivers and battery charge regulators, are not being targeted for replacement by the current generation of cells. Neither are the the peripherals, such as motors, temperature sensors, and batteries. Nevertheless, as will be shown throughout the rest of this thesis, the fraction of avionics which can be replaced by the cells described in this chapter, is significant. 
Artificial Cell

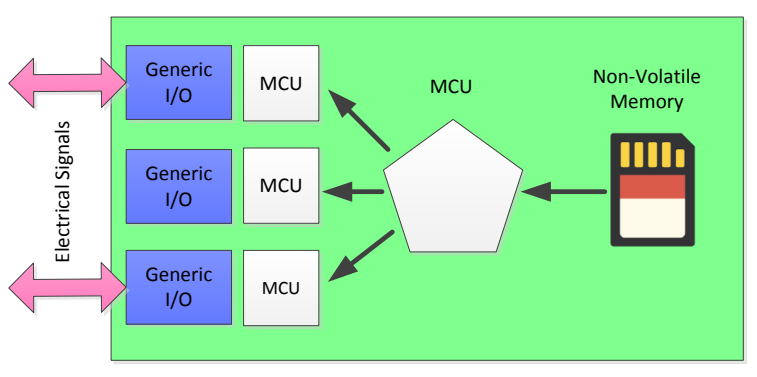

Biological Cell

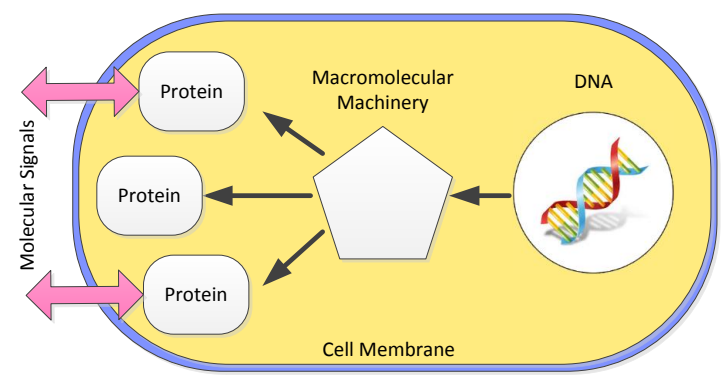

Figure 4.1: A simplified depiction of the differentiation process in a biological cell, and the proposed artificial implementation.

\subsection{Artificial vs Biological Cells}

Figure 4.1 shows a simplified representation of the inner workings of a biological cell, focussing specifically on the process of gene expression. As explained in detail in Chapter 3 , it is the process of gene expression which allows a single biological cell to differentiate into any of the specialised cells required within an organism. It is this quality, of subsystems being able to reconfigure to perform a variety of roles, which lies at the heart of ASC Architecture. Thus, as shown in Figure 4.1, an artificial cell capable of undergoing a process similar to gene expression is proposed.

In a biological cell the majority of cellular tasks are performed by proteins. Proteins are complex molecules and each one exists to perform a specific function. Proteins can interact with one another directly, or with the outside world. The interface to the outside world is provided by the cell membrane, which also acts to protect the cell's internals from the environment. At any one time, a cell will contain a set of different proteins, and it is this set which determines the cell's functionality.

In the proposed artificial cell the role of the proteins is performed by a set of discrete processing elements. It is envisioned that these will either be MCUs or FPGAs. The discrete nature of the processing elements is important to aid reliability, as the failure of a single protein should not interfere with other proteins. Before being configured by having its firmware or bitstream programmed, a processing element can be seen as a blank protein slot, or a protein skeleton. A processing element is capable of pure computational tasks, or, through the use of generic I/O circuitry, tasks which require external interfaces to sensors or actuators. The generic I/O circuitry, which is explained in detail in Section 4.2, is analogous to the cell membrane. 
In a biological cell proteins are created through the processes of transcription and translation by a set of components known as the macromolecular machinery. These processes are described in detail in Chapter 3, but in essence involve synthesising proteins from blueprints stored in the DNA. Every cell in an organism contains the same DNA, which encodes instructions for synthesising every possible protein needed by the organism. The macromolecular machinery is sensitive to conditions internal (e.g. protein concentrations and energy levels) and external (e.g. messenger molecule concentrations) to the cell and varies which proteins are being synthesised at any one time in response. In this way, a cell can react to changing environmental conditions by changing its set of proteins, and therefore its capabilities.

The artificial cell's version of protein synthesis involves programming a blank protein processing element with a specific firmware image or bitstream, which is stored, with others, in non-volatile memory. Every artificial cell in a system contains the same set of programming files on board. While this may seem wasteful of memory, non-volatile memory technologies, such as flash, have progressed sufficiently that the physical and budgetary costs of memory capacity are not of large concern in this application. The macromolecular machinery's role is performed by a dedicated MCU on each cell. This MCU reacts to internal and external conditions by loading specific programming files onto its proteins. The primary monitored internal condition is the health and status of individual proteins. A likely response of the macromolecular machinery to a failed protein is to reprogram a blank protein with the lost task. Examples of actioned external conditions include changing available power levels and increased radiation levels.

As each biological protein is essentially custom-built for a specific task, so, too, each artificial protein program should be designed to execute a single system task. Thus, the system designer is forced to break down the overall system functionality into tasks small enough to be performed by individual proteins. The granularity of these tasks is dependent on the processing power of the protein processing elements. Here, a trade-off exists. Smaller tasks aid robustness, as the failure of an individual protein will have a less noticeable effect on the system, whether or not that task is later taken up by another protein. However, as tasks are made simpler and their number increases, the inter-protein communication bandwidth is likely to increase. Additionally, some system tasks may be difficult or inefficient to break down further. Thus, the choice of protein processing elements should ideally only be made after careful consideration of the intended system tasks. 
Table 4.1: The interfaces of typical satellite sensors and actuators.

\begin{tabular}{|l|c|l|c|}
\hline Peripheral & Number on Sat & Interface per Device & I/O Channels \\
\hline Reaction Wheel & $0-3$ & $\begin{array}{l}3 \text { digital inputs } \\
3 \text { half-bridge outputs } \\
1 \text { digital power }\end{array}$ & 7 \\
\hline Magetorquer & 3 & 2 half-bridge outputs & 2 \\
\hline Burn Wire & $0-3$ & 1 half-bridge output & 1 \\
\hline Magnetometer & 1 & $\begin{array}{l}1 \text { digital power output } \\
1 \text { half-bridge output } \\
6 \text { analogue inputs } \\
1 \text { digital power output } \\
1 \text { analogue input }\end{array}$ & 8 \\
\hline Temperature Sensor & $3-10$ & $\begin{array}{l}1 \text { digital power output } \\
2 \text { digital I/Os }\end{array}$ & 3 \\
\hline MEMs Gyro & $1-3$ & 1 analogue input & 1 \\
\hline Coarse Sun Sensor & 6 & & \\
\hline
\end{tabular}

\subsection{Artificial Cell Membranes and Peripheral Interfacing}

Having the ability to interface directly to external peripherals is a vital characteristic of the Satellite Stem Cell Architecture's cells. This feature sets the architecture apart from other modular, distributed processing architectures, and allows cells to directly replace sections of traditional satellite avionics.

There are two major challenges in designing the generic I/O circuitry of the artificial cell's membrane. Firstly, there is the problem of making the I/O circuitry compatible with the wide variety of interfaces commonly seen in spacecraft avionics. As seen in Table 4.1, which lists some common sensors and actuators, the required interfaces include high power outputs, analogue inputs, and communication buses. Secondly, there is the problem of how to make interfaces to peripherals robust and redundant.

To solve the first problem, a variation of the I/O circuitry commonly found in MCUs is proposed. This is shown in simplified form in Figure 4.2. An input stage, output stage and protection circuitry are combined to form a single I/O channel. As is the case in most MCUs, the output stage is based on a MOSFET half-bridge. However, unlike most MCU outputs which are only capable of sourcing tens of milliamps, the proposed half-bridge is capable of supplying amps of current to directly drive actuators such as torquers and motors. In addition, while the outputs of most MCUs are limited to operation at the $3 \mathrm{~V} / 5 \mathrm{~V}$ supply rails of the MCU itself, the proposed half-bridge will accept alternative drive voltages. 


\subsection{Artificial Cell Membranes and Peripheral Interfacing}

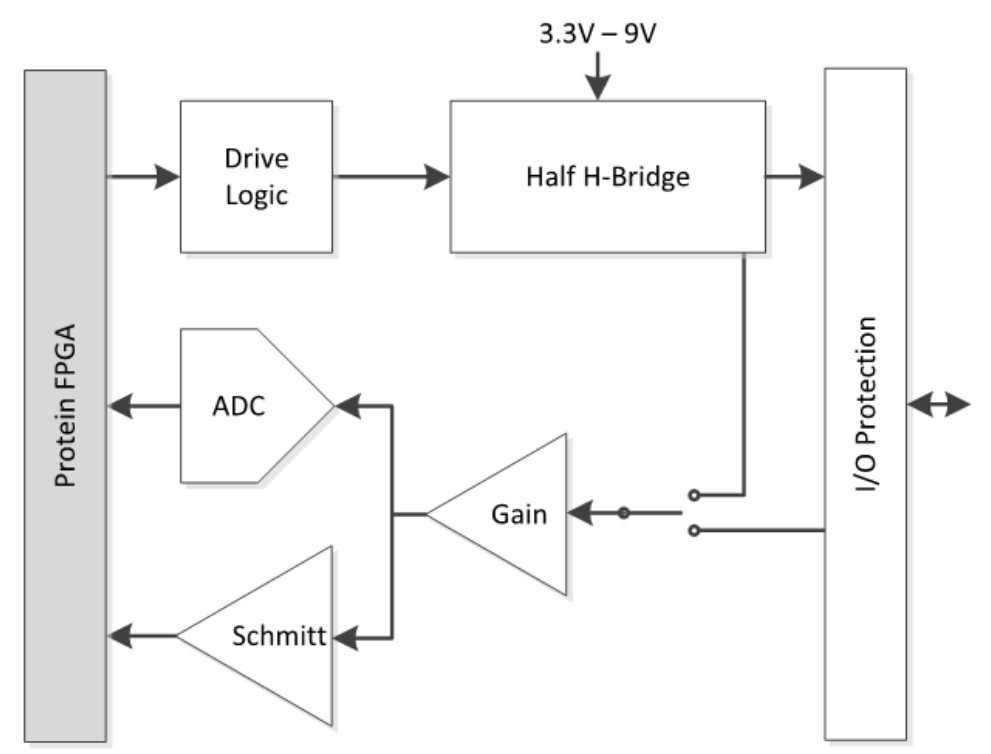

Figure 4.2: Block diagram of the generic I/O circuitry which allows proteins to interface directly to a variety of external peripherals.

The proposed input stage is based on an analogue to digital converter and schmitt trigger, similar to typical MCU input stages. However, in addition, the input stage features adjustable gain and the ability to switch between measuring an input voltage and measuring the current being delivered by the output stage.

Finally, the protection circuitry is responsible for preventing unexpected voltages on the I/O channel from damaging the rest of the circuitry. There will be limits to the protection offered, but it must at least protect against electrostatic discharge during handling.

To solve the problem of making peripheral interfaces robust and redundant, three options were considered. The first option, depicted in Figure 4.3, involves having a bank of I/O channels which can be controlled by multiple proteins through a set of digital multiplexers (muxes). This configuration could theoretically allow any protein MCU on a cell to interact with any peripheral connected to that cell's I/O channels. However, this solution has several disadvantages. Firstly, the I/O channels connecting to a particular peripheral are not redundant. Therefore, if the I/O circuitry (or the associated mux) fails the attached peripheral can no longer be accessed. Secondly, while multiple proteins on a single cell can access the same peripherals, no cross-strapping between cells is possible. Finally, there is the problem of who controls the muxes. No single protein may be the sole controller of a mux, and multiplexing control of the muxes is likely to become infeasibly complex. 


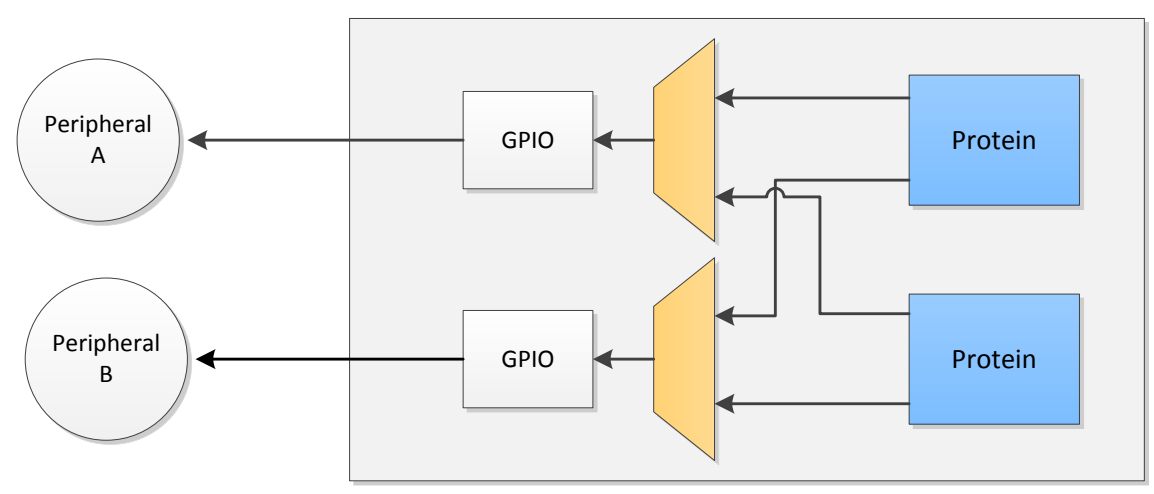

Figure 4.3: The first peripheral interfacing scheme gives each cell a set of communal I/O channels, which can be accessed by multiple proteins on the same cell through multiplexers.

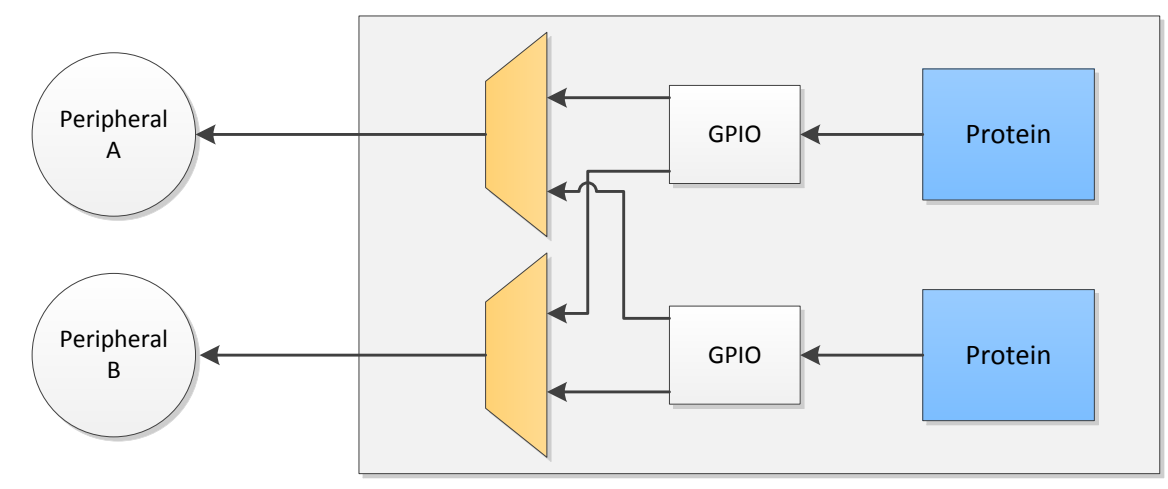

Figure 4.4: The second peripheral interfacing scheme gives each protein its own I/O channels, which can be switched onto different peripherals using multiplexers.

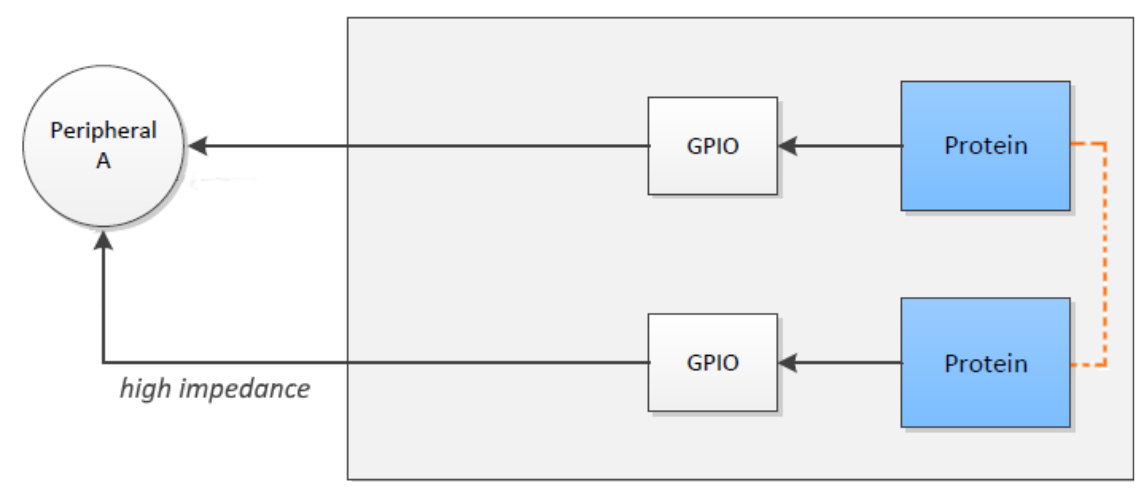

Figure 4.5: The third peripheral interfacing scheme removes multiplexers altogether. Instead, I/O signal conflicts are avoided through a high-impedance mode I/O mode and through inter-protein cooperation (in software). 


\subsection{Artificial Cell Membranes and Peripheral Interfacing}

The second option, depicted in Figure 4.4, replaces the digital muxes with analogue ones and moves them to the other side of the I/O channels. In this configuration, every protein has its own set of I/O channels and every peripheral is assigned a mux. Through switching of the mux, a peripheral can be controlled by various proteins. The primary advantage of this solution is the redundancy of the I/O circuitry in each peripherals interface. However, there are also multiple disadvantages. As before, the control of the muxes remains an unresolved issue, and due to the need for physically housing the mux on a cell, cross-strapping between cells is still impossible. In addition, the location of the muxes in this configuration requires them to potentially pass large amounts of current, as would be the case if the attached peripheral is a high-powered actuator. While analogue mux ICs capable of transmitting a few milliamps are common, a high-current version would likely have to be built out of discrete components. Such a mux would be unfavourably large and complex. Finally, while every peripheral may be controllable by several proteins, each individual protein is likely to have access to only a single, or few, peripherals. This is because every additional peripheral a protein is given access to requires additional I/O channels from the protein. Every I/O channel comes with overheads, so their number should be limited.

The third option, depicted in Figure 4.5, removes muxes altogether. As in the second option, in this configuration each protein has its own set of I/O channels. However, instead of going via a mux, the I/O circuitry is connected directly to a peripheral. By equipping the I/O circuitry with a special high-impedance mode, multiple proteins' I/O channels can be directly connected to the same peripheral. All the proteins not currently interacting with their attached peripherals put their output channels into high-impedance mode, which effectively disconnects them from the peripherals. As before, every peripheral can be interfaced to multiple proteins, but in this case each protein has access to only a single peripheral. This mux-less configuration offers two main advantages. Firstly, peripherals can be arbitrarily cross-strapped between proteins of different cells (not just between proteins on the same cell). Secondly, the I/O circuitry is fully redundant in each peripheral interface.

However, while the problem of who controls the muxes is removed, the issue of peripheral access control still exists. With no mux guaranteeing sole access, multiple sets of GPIO circuitry can interact in undesireable ways. For example, two sets can compete, with one set attempting to drive a line high, while another drives it low. Alternatively, one set of GPIO circuitry may fail in such a way that it holds the line high or low.

The first situation must be dealt with in software. The cells must be capable of communicating with one another to ensure that each peripheral is only being driven by a single protein at once. This communication is described in Chapter 4. Arbitration between proteins attempting 
to access the same peripheral could be further aided by the fact that every I/O channel can serve as both an input and an output, potentially allowing a protein to detect if another protein is currently driving it's attached peripheral.

The second situation, involving failed GPIO channels disabling each other, must be prevented through good hardware design. A GPIO channel will not interfere with another if it is in a high-impedance state. Therefore, the GPIO circuitry must be designed with well-understood failure modes, which, as far as possible, result in high-independence states. Using fault tree analysis and mostly discrete components, such a design is developed in Section 7.2.1).

The reliability of an interfacing scheme is based on a combination of its reconfigurability and circuitry reliability. Of the three interfacing schemes considered, option three represents the best compromise between these two characteristics. The fact that each protein can only be interfaced to a single peripheral limits the reconfiguarability of multicellular systems based on this scheme. However, this disadvantage is offset by being the only option which allows inter-cell cross-strapping, and does not require muxes (which are difficult to find off the shelf with the correct specifications and require 'centralised' control). Thus, the cells of the Artificial Stem Cell Architecture implement interfacing scheme three.

Having chosen the mux-less peripheral interfacing option, an important design question is remains: How many I/O channels does each set of GPIO circuitry provide? As seen in Table 4.1, for the sensors and actuators commonly found on a satellite, one to eight I/O channels are required. Two channels are sufficient for driving a simple actuator such as a magnetorquer or for reading a simple analogue sensor. In contrast, seven I/O channels are required for controlling a brushless-DC motor and eight are required for interfacing to a mixed-signal magnetometer (ground return is not included in these counts as it can bypass the I/O channel and go directly to satellite ground). While the number of I/O channels dictates the type of peripherals that can be interfaced to, it does not limit the number of proteins the same peripheral could be cross-strapped to.

The maximum level of cross-strapping will be dependent on the I/O circuitry implementation and specific peripheral electrical requirements. For example, communication buses such as I2C specify a maximum bus capacitance, which will increase with each additional interfaced I/O channel. Peripherals can also be cross-strapped between cells.

Deciding on the number of I/O channels per protein is left as a design parameter for the developer, together with the number of proteins per cell and cells per system. To aid in making these decisions, Chapter 6 examines the effect of these parameters on system reliability and makes recommendations based on a number of observed trends in system behaviour. 


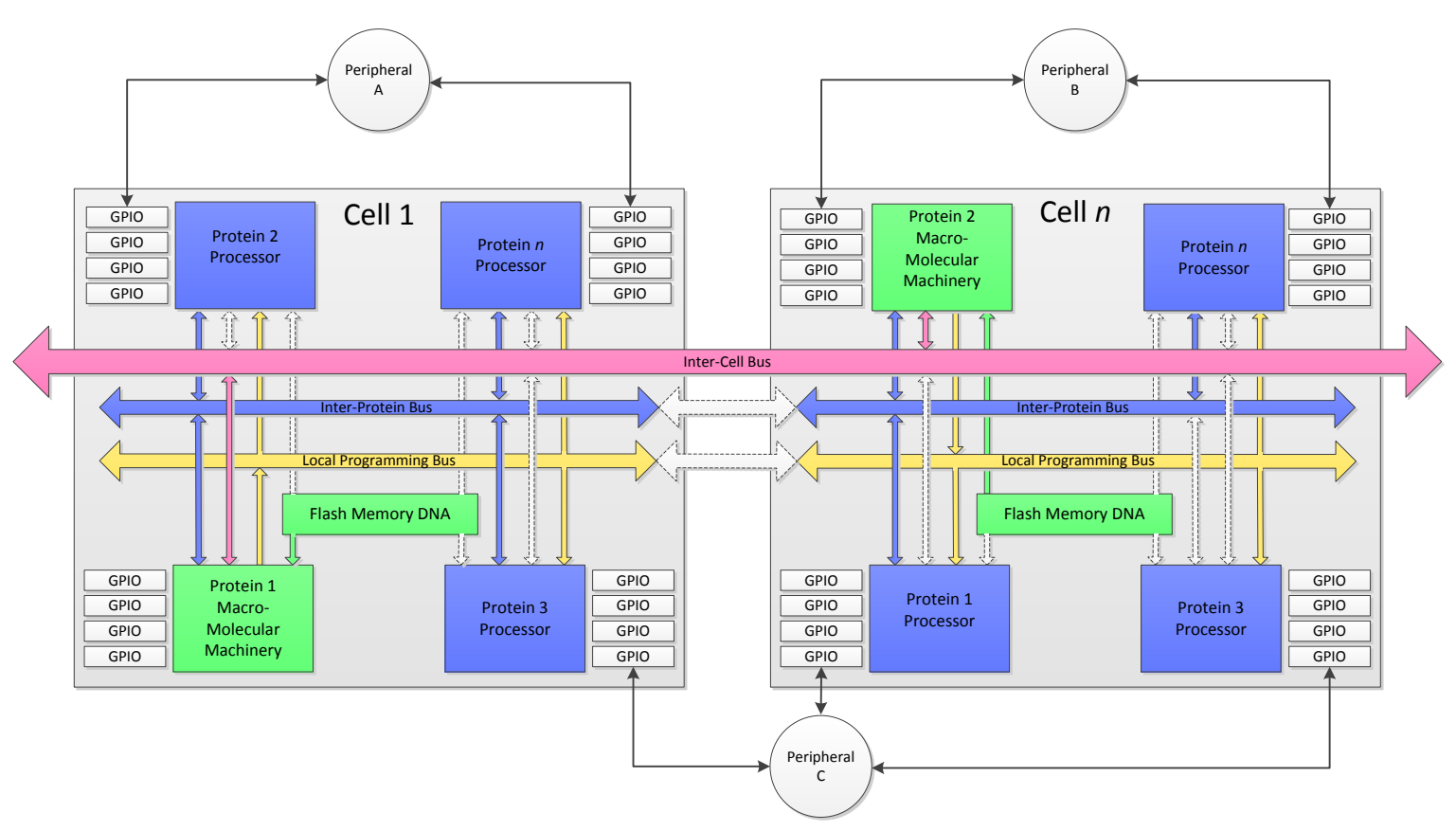

Figure 4.6: The detailed design of two artificial cells within a multicellular system. Any protein on a cell can take on the role of macro-molecular machinery. Peripherals can be cross-strapped to an arbitrary number of proteins, as determined by the peripheral's criticality. In addition, peripherals can be cross-strapped between cells.

\subsection{A Multicellular System}

At this point the design of the proposed artificial cell and its functioning within a system of cells can be further defined. While Figure 4.1 served to illustrate the comparison between the proposed artificial cell and a biological cell, a more detailed design is required. This is given in Figure 4.6, which shows the internal structure of two artificial cells from within a multicellular architecture.

The first thing to notice about the cell's design is is symmetrical nature. Every protein has its own I/O channels and is interfaced to other proteins using three communications buses. There is no dedicated macromolecular machinery MCU. Instead, any of the proteins have the ability to take on the role of macromolecular machinery. This design is not far removed from its biological counterpart, as the macromolecular machinery found inside biological cells is itself largely composed of proteins. The protein acting as MM does not utilise its I/O channels, so it does not interfere with peripherals cross-strapped to it. 


\subsubsection{Inter-Protein and Inter-Cell Communication}

In Figure 4.6, the proteins are shown as being linked with three communication buses, namely, an internal inter-protein bus, an internal programming bus, and a bus for inter-cell communication. Any protein on a cell can send and receive messages on the inter-protein bus, which is analogous to the medium within biological cells through which messenger molecules are able to move freely. In contrast, only the protein currently acting as macromolecular machinery is allowed access to the inter-cellular bus. Any protein needing to send messages to other cells must send them via the macromolecular machinery protein, which acts as a router. Thus, in the artificial cell, the MM protein takes on the role of message access control which is performed by the cell membrane in biological cells. Compared to putting all proteins from all cells on the same bus, this design offers two key advantages. Firstly, on average, the traffic on both the internal and inter-cellular buses is reduced. Messages which originate and terminate on the same cell do not appear on the inter-cellular bus. Secondly, this design allows a misbehaving protein to be isolated from the rest of the system. The MM protein can look out for 'babbling-idiot' failure modes and block such messages from corrupting the rest of the system.

The programming bus and DNA memory are also controlled solely by the MM protein. By reading programming files from the memory and reprogramming proteins over the programming bus, the MM protein is able to emulate the process of gene expression. The MM protein is constantly monitoring the health of the other proteins on its cell through polling. If it detects a failure, it is able to reprogram a blank protein to take up the lost task, or it can reprogram an active, but lower priority protein.

\subsubsection{Artificial Mitosis}

An important failure mode is the failure of the MM protein. In this case, one of the other proteins on the cell must take over the role of MM. In order for this to be possible, every protein must have electrical access to all three of the buses and the DNA memory, even if only the currently active MM protein is allowed access to them. It is undesirable to have every protein on a cell preprogrammed with the ability to be the MM, as it complicates the process of writing 'user software'. Ideally, developers writing user code, which is designed to perform system functions and intended to be executed on proteins, should not have to concern themselves with the system reconfiguration process. It should happen transparently. Thus, upon failure of a cell's MM protein, one of the remaining proteins needs to be reprogrammed 
into the new MM. This is achieved by having the MM of another cell in the system reprogram a protein on the stricken cell. This process can superficially be compared to cell mitosis.

In a simplified sense, during mitosis, a biological cell splits in two, resulting in two fully functional cells. In the case of the proposed artificial cells, once the MM fails the cell could be described as being dead. At this point, a watchdog timer times out and activates a bridge which connects the dead cell's programming and inter-protein buses to the corresponding buses on its neighbouring cell. From the point of view of the neighbouring cell's MM, it is still a single cell but now with more proteins under its control. This is the point at which artificial mitosis starts. The MM chooses one of its new proteins (which is physically on the dead cell) and reprograms it with the MM code. Once the new MM boots, it disconnects its cell's buses from its neighbouring cell and proceeds to operate as usual.

\subsubsection{Cell Death}

If an artificial cell is misbehaving and negatively affecting other cells in the system, it may need to be isolated. In the majority of cases, the cell's ill behaviour will be due to a misbehaving protein. This can be dealt with by the MM, which can turn off specific proteins. How a misbehaving protein is detected is not specifically defined, but the MM may be able to monitor its power consumption, responses to specific queries, and reset/stall rates.

If the MM protein itself is misbehaving, the only way in which it will be detected is if the watchdog timer times out and a neighbouring cell intervenes, as described in Section 4.3.2. Ideally, other cells in the system should be able to 'vote out' a misbehaving cell, causing it to isolate itself. However, provided the MM protein is misbehaving, the current generation cell design does not include such a mechanism.

\subsection{Summary}

This chapter described the detailed design of an artificial cell, which serves as the building block of systems based on the Satellite Stem Cell architecture.

Following the conceptual, multicellular system outline developed in Chapter 3, the artificial cell must be mass-manufacturable, but also capable of performing a wide variety of tasks. To this end, the artificial cell mimics the biological processes of protein synthesis and differentiation. Discrete processing elements take the place of proteins and perform user 


\section{Artificial Stem Cells}

defined tasks, while an additional processor performs the role of macromolecular machinery by reprogramming the processors with firmware stored in non-volatile 'DNA' memory.

By observing that the macromolecular machinery itself is largely composed of proteins, the artificial cell could be simplified to include only one type of processing element. Thus, at any one time, one processor on every cell is devoted to the macrocolecular machinery task, while the rest are free to execute user-defined tasks. Both the protein tasks, and the macromolecular machinery task, can be moved in the event of partial hardware failures.

To progress beyond pure computational systems and enable complex sensor-actuator systems to be developed without additional hardware, a set of novel, generic I/O circuitry was developed and included in each cell. Each generic I/O channel can serve as a digital or analogue input, as well as a high-power output. Furthermore, each I/O channel has a highimpedance mode, which allows peripherals to be cross-strapped between several channels. This solution avoids the complexity of multiplexers, while ensuring that the drive electronics are redundant.

Ideally, the artificial cells described in this chapter will be mass produced, as each cell is electrically identical. To combine a set of cells into a complete system, the cells are linked together using a common communication bus, peripherals are interfaced to the generic $\mathrm{I} / \mathrm{O}$ channels and cross-strapped to a user-defined level, and each cell's non-volatile memory is programmed with a complete list of firmware for executing all system tasks. Additional redundancy, or processing capacity, can still be added late in the development by simply adding additional cells.

A physical implementation of an artificial cell, based on the architecture given in this chapter, is described in Chapter 7, together with measurements of performance and overheads. 


\section{Chapter 5}

\section{Hybrid Agent Real-Time Platform}

The complex processes which take place within a cell, including gene expression, differentiation and the initial phases of mitosis, require careful orchestration. Furthermore, for a multicellular organism to be successful, not only must its individual cells operate correctly, but these cells are also required to interact and cooperate with one another as part of a global task management scheme.

The artificial cells of the Artificial Stem Cell Architecture, described in Chapter 4, have the required hardware to perform artificial versions of these important internal and intercellular processes. However, it is desirable to abstract the hardware and the execution of these processes from the application developer. Ideally, application code should be executed robustly and transparently on the proteins of a multicellular system, without the application developer having to be familiar with the process by which the code got to the specific protein processors. To this end, the Hybrid Agent Real-Time Platform (HARP), a novel software platform which combines real-time capabilities with the power and intuitiveness of agent computing, has been developed.

The concept of agent computing emerged in the 1990s as a new, high-level programming philosophy. A software agent is a program which can make autonomous decisions within its environment to meet its design objectives. A software agent has aims, a life-cycle and, in some cases, the ability to migrate between systems. Agents typically execute within a software framework called an agency, which provides supporting services, such as agent creation, life cycle management and inter-agency communication routing. The similarities between the operation of an agent within an agency, and the operation of proteins within a cell, have guided the development of the HARP middleware. Thus, the HARP middleware 

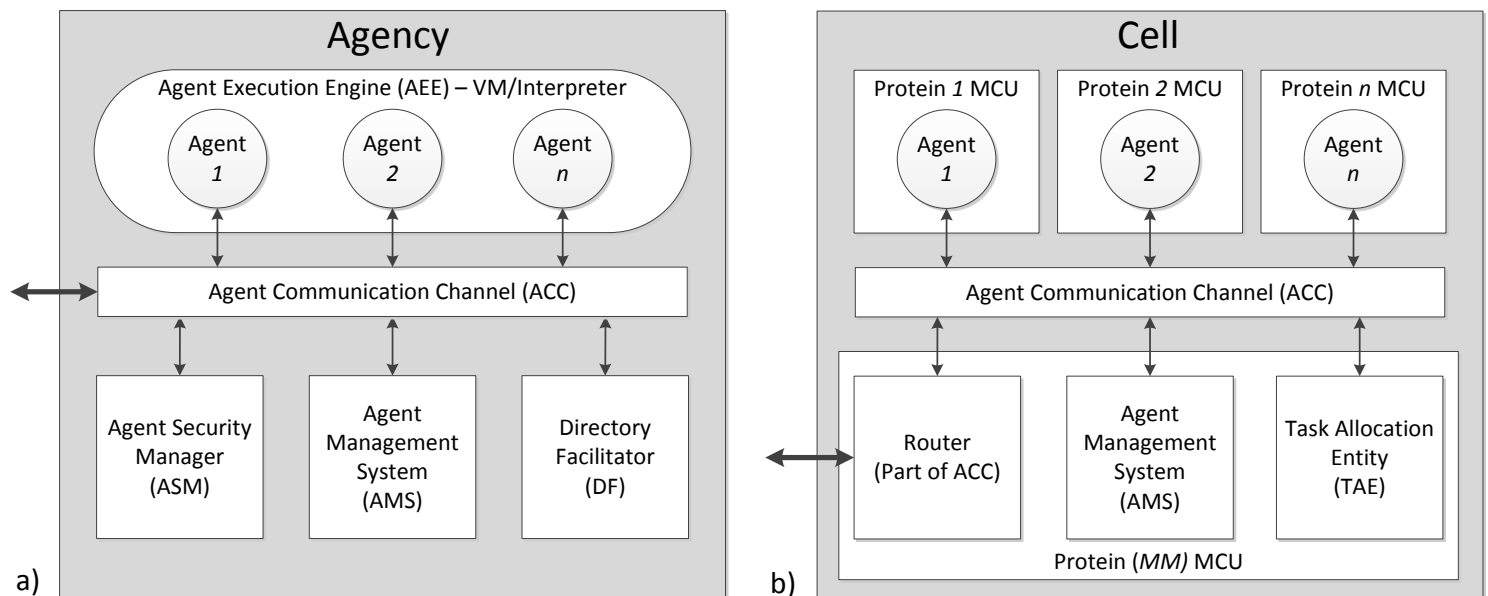

Figure 5.1: Schematic representation of an agency based on the FIPA Abstract Archtecture (a), and an agent platform based on the HARP middleware running on an artificial stem cell (b). Note that the Directory Facilitator is an optional component which is not currently implemented in the HARP middleware.

turns every artificial cell into an agency, which supports the execution of 'protein' agents on the hardware protein processors.

In this chapter, the core components of the HARP middleware will be described, along with unique, extended features relating to peer-to-peer task allocation, real-time capabilities, and fault tolerance.

\subsection{FIPA Abstract Architecture}

The HARP middleware is based on the FIPA Abstract Architecture [92]. The FIPA organisation was formed to promote the interoperation of agents from different platforms. To this end, they released the FIPA Abstract Architecture, which defines the fundamental components required of any compatible agent platform, as well as specifying how agents should interact with these components and each other. The components of a FIPA-compatible platform include an Agent Execution Environment (AEE), Agent Management System (AMS) and Agent Communication Channel (ACC). A schematic representation of the FIPA Abstract Architecture and the HARP middleware are shown in Figure 5.1, and will be referred to throughout this chapter. 


\subsubsection{Agent Execution Environment}

Every agent platform has an Agent Execution Environment at its core. The AEE is responsible for executing agent code on a host machine and for maintaining a security policy to ensure that agents cannot interfere with one another or the host machine. To enable agent mobility, AEEs are often designed to present a uniform execution environment, even across different hardware platforms. To this end, AEEs are commonly implemented using virtual environments, interpreters, or virtual machines. Examples include the JADE AEE, which is based on a Java virtual machine, or the Mobile-C AEE, which is based on a $\mathrm{C}$ interpreter.

Due to the real-time requirements and limited processing resources of satellite platforms, the HARP middleware's AEE is not based on a virtual machine or interpreter. Instead, protein agents are executed as native code on discrete processors. As described in Chapter 4, the artificial cells of the ASC Architecture each contain a number of discrete protein processors. While one of these is always reserved for macromolecular machinery duty, the rest are free to execute protein agent code. This is depicted in Figure 5.1b. The use of discrete processors executing native code has several advantages. These include more efficient code execution (and therefore lower power consumption) and the intrinsic ability to implement real-time tasks, which are essential for AOCS operations. Because agent code is pre-compiled before it is deployed onto the system, agent mobility between platforms based different processors is not supported. However, just as proteins can migrate between the cells of a biological organism, the agent proteins can migrate between the artificial cells of a multicellular system. This process is orchestrated by the Agent Management System.

\subsubsection{Agent Management System}

The Agent Management System is analogous to a cell's macromolecular machinery. It is responsible for managing the lifecycles of protein agents, including their creation, registration, decommissioning, and migration. Since every artificial cell is treated as an agency, every cell has its own AMS, which is executed on the macromolecular machinery protein. To create a new agent the AMS implements an artificial version of gene expression by reading a set of compiled agent code from DNA memory and programming it onto one of its protein processors. According to the FIPA Abstract Architecture, every agent is required to register with its AMS, which it does using a unique, hard-coded, user-defined identifier. The AMS maintains a list, called the agent directory, of all the protein agents currently executing on its 
cell. The AMS provides a service which allows the agent directory to be queried by proteins on the same cell, or by proteins on other cells, to look-up communication addresses.

Going beyond the basic requirements of a FIPA AMS, a HARP AMS continuously monitors the health of all its registered agents. This is done by measuring the current consumption of their respective protein processors and by checking their responsiveness. If an agent fails its health check, the AMS will attempt to revive it. This process may involve several steps, including soft and hard restarts (power cycling the protein processor) or reprogramming. If it is determined that an the protein processor on which an agent was executing has suffered an unrecoverable failure, the AMS can attempt to restore the agent on another protein processor.

The process of agent restoration is the same as agent migration. The ability for agents to migrate is not a compulsory feature of FIPA-compatible platforms. Nevertheless, many agent platforms support some form of agent migration. To differentiate migration from simply creating a new agent of the same type in another location, migration requires the continuity of some level of runtime information. The strictest implementations transfer the instruction counter and stack. The HARP implementation only provides the ability to transfer user defined runtime variables. While executing, an agent may request its AMS to store given runtime variables in central, volatile memory. This memory is different from the DNA memory as it is non-volatile. It represents the intra-cellular medium within a biological cell in which cell state is effectively encoded through the specific concentrations of proteins and messenger molecules. The AMS's Agent Directory is stored in the same memory.

To restore an agent, the AMS effectively creates a new agent of the same type on an available protein processor. Alternatively, the AMS can request another cell's AMS to create the new agent. It is then up to the newly created agent to query the AMS for any available runtime variables.

\subsubsection{Agent Communication Channel}

To facilitate communication between agents and the AMS, and between agents themselves, the FIPA Abstract Architecture specifies the need for an Agent Communication Channel and Agent Communication Language (ACL). The ACC interacts with other agency services, such as the AMS Agent Directory, to provide a Message Transport Service (MTS). Since effective inter-agent communication is a key goal of the FIPA organisation, it provides extensive specifications relating to, amongst others, transport protocols, message encoding and agent 


\begin{tabular}{|c|}
\hline Application \\
\hline Presentation \\
\hline Session \\
\hline Transport \\
\hline Network \\
\hline Data Link \\
\hline Physical \\
\hline
\end{tabular}

OSI Network Stack

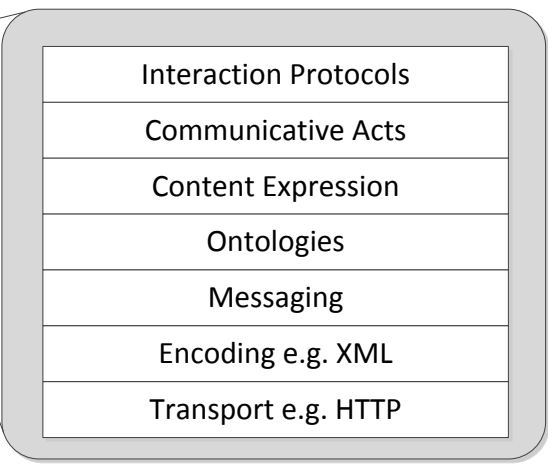

FIPA ACL Protocol Stack

Figure 5.2: The relationship between the OSI network stack and the FIPA communication stack.

\begin{tabular}{|c|c|}
\hline FIPA Interaction Protocols & Request, Query \\
\hline FIPA Communicative Acts & $\begin{array}{c}\text { request, query-ref, refuse, agree, } \\
\text { failure, inform-done, inform-result, } \\
\text { not-understood }\end{array}$ \\
\hline FIPA Messaging & ACL \\
\hline FIPA Encoding & Bit-Efficient \\
\hline Transport & ISO-TP \\
\hline Network & CAN 2.0 \\
\hline Data Link & \\
\hline Physical & \\
\hline
\end{tabular}

Figure 5.3: The HARP middleware communication stack, supporting both ACL and real-time messaging. Layers shown in grey implement FIPA protocols.

interaction protocols. However, FIPA does not concern itself with the lower levels of the Open Systems Interconnect (OSI) network stack [93]. The FIPA communications model is is a service oriented model and is described using its own protocol stack which resides within the application layer of the OSI network stack [94]. This relationship is shown in Figure 5.2 .

The HARP ACC's message transfer service does not implement a FIPA-defined message transfer protocol and is therefore not fully FIPA compliant. However, it does implement the higher levels of the FIPA communications stack, including the encoding, messaging, communicative acts and interaction protocols. This design, shown in Figure 5.3, allows both FIPA ACL messages and real-time packets to be sent between agents. 


\subsubsection{Physical and Data Link Layers}

The physical and data link layers of the HARP communications stack are based on CAN 2.0A. CAN is a multi-master bus which is used extensively in real-time, multi-master environments, such as vehicles and automated production lines. The choice of CAN for the HARP communication stack was made in collaboration with the artificial cell hardware development. The Artificial Stem Cell Architecture calls for peer-to-peer networking between its proteins and cells, necessitating a multi-master bus. CAN was chosen for its flight heritage, real-time messaging capabilities, and low implementation cost in terms of processing power, power consumption, and volume.

Flight heritage comes from SSTL, amongst others, who have successfully used CAN as their main spacecraft bus [95]. CAN's real-time capabilities derive from its deterministic message arbitration scheme [96]. A CAN frame is composed of several fields, including a message identifier (ID), payload data, a cyclic redundancy check (CRC) code and various other flow control bits. Arbitration between messages from different masters is performed based on the message ID, with lower numerical IDs receiving priority. Thus, a node attempting to send a low priority message, which gets suppressed by a higher priority message, will attempt to retransmit.

In a CAN network, the arbitration, message framing, acknowledgement and error detection are orchestrated in hardware by discrete CAN controller and transceiver ICs. These make implementation simple and free up valuable processor time. In fact, an increasing number of MCUs are becoming available with the CAN controller, and even the transceiver, embedded as a peripherals.

A CAN frame does not necessarily contain any information about its origin or destination. All it has is a message ID, for which receivers can selectively filter. Thus, a CAN network can be set up to use the message IDs as destination addresses, or IDs can be used to indicate message contents. As an example, the ID could be used to indicate whether a message's contents were temperature, battery voltage, or attitude telemetry. However, because message IDs are used for arbitration, it is important that each node on a CAN bus uses a unique set of IDs.

\subsubsection{Network and Transport Layers}

In the HARP communication stack, the CAN message IDs are used as destination addresses. Every active protein in the system has a unique address which is composed of a 4-bit cell 
address and 7-bit protein address. Together, these form the standard 11-bit CAN message ID field. A protein is given its address, which is based on its physical location within the system, when it registers with its AMS. In addition, certain IDs are reserved for special functions. Examples include protein ID 127, which is reserved for AMSs and cell ID 0, which is reserved for real-time traffic.

Every cell has an internal, inter-protein bus and is connected to the global, inter-cellular bus. CAN frames are routed between these two buses by a router service, which is executed on the MM protein. The router service uses the message IDs to determine whether CAN frames need to be routed into, or out of, the cell. On the internal bus the router service filters for messages with cell addresses which do not match the local cell address. Such frames are repeated onto the inter-cellular bus. Similarly, on the inter-cellular bus the router service filters for messages with a cell address which matches the local cell address. Such frames are repeated onto the internal bus. Thus, the router service does not need to have any understanding of the higher level FIPA protocols. It could, however, be instructed not to route certain message IDs to isolate misbehaving proteins from the rest of the system.

Standard CAN frames have a maximum payload capability of 8 bytes. This is insufficient for implementing higher-level FIPA protocols. Thus, an additional protocol is required which can package longer messages into a set of standard CAN frames. A suitable protocol is ISO 15765-2, or ISO-TP, which is commonly used for transporting diagnostic messages across a vehicles CAN network. ISO-TP sacrifices one or more of the payload bytes in each standard CAN frame to encode information, called the protocol control information (PCI), which allow multiple frames to be recombined. ISO-TP defines four different frame types, each of which is identified using the PCI. These types are called single, first, consecutive and flow control frames.

Messages which are shorter than eight bytes are sent as a single frame. The transfer of a longer message begins with the transmission of a first frame, which contains the first six bytes of message data and PCI describing the frame type and total message length. Thereafter, the sender waits for a flow control frame from the recipient, which specifies parameters for the transmission of further frames. These parameters include the number of frames which can be sent before waiting for another flow control frame, and the rate of transmission of frames. Upon reception of a flow control frame, the sender completes the transaction by sending a series of consecutive frames, each of which contains seven bytes of message data.

ISO-TP enables the transfer of messages which are longer than eight bytes, which is essential for implementing higher-level FIPA protocols. However, in its standard form, it is not a capable ACL transport service as its packets do not contain sender information. Thus, the 

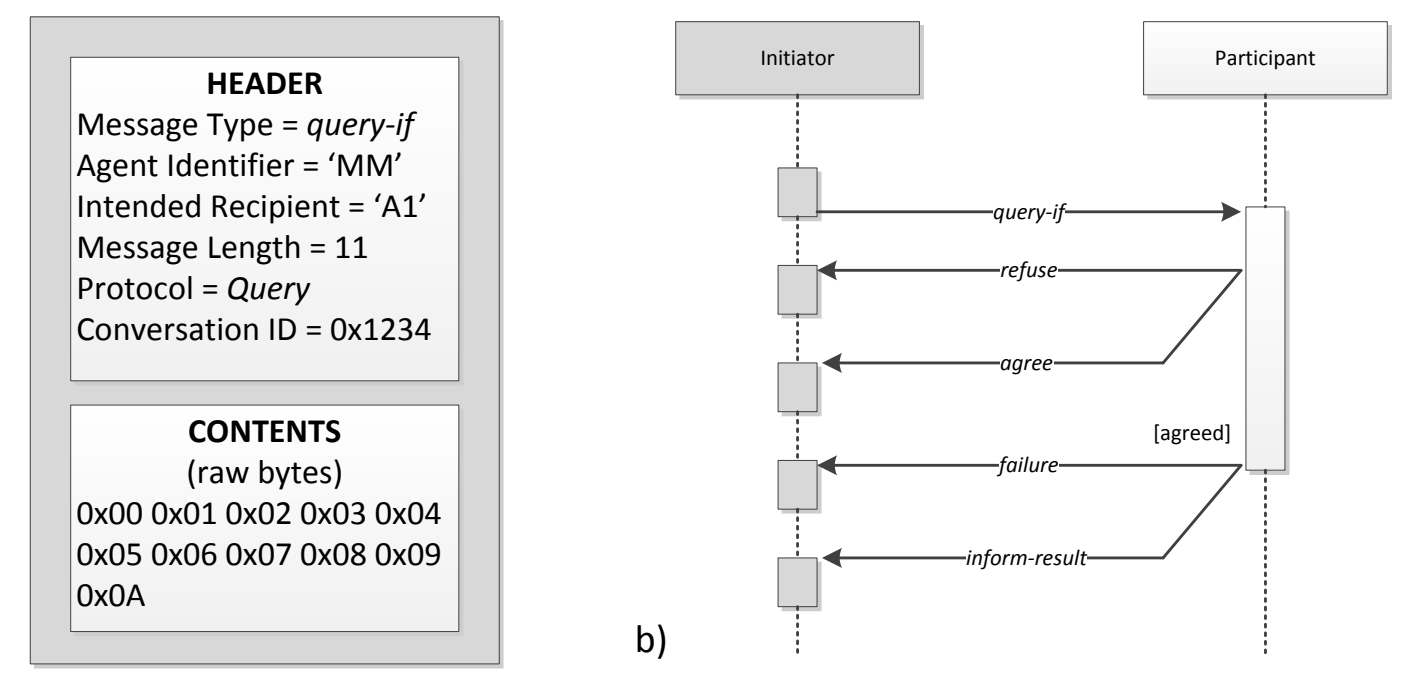

Figure 5.4: An example HARP ACL message (a), and a schematic representation of the Query FIPA interaction protocol.

ISO-TP protocol was modified slightly to include the sender address in the PCI of first frames. This reduces the number of message payload bytes in first frames from six to four, but does not impact consecutive frames.

\subsubsection{FIPA Higher-Level Protocols}

The ISO-TP protocol is used to transport FIPA ACL messages. FIPA ACL messages are designed to be universally understood by FIPA-compatible agents, and as such contain information about the message format, its origin, and destination in a header. The specifications for FIPA ACL messages describe what fields must be present in the header, as well as specifying several optional fields. An example ACL message, as used by the agents of the HARP middleware, is shown in Figure 5.4a. The Agent Identifier, Intended Recipient, and Message Length header fields are self-explanatory. The purposes of the Message Type, Protocol, and Conversation ID fields will become clear once the various layers of the FIPA protocol stack have been described.

In addition to specifying the structure of ACL messages, FIPA also has specifications for how these messages should be encoded for transport. Several encoding schemes are specified, including XML, String, and Bit-Efficient. The XML and String encodings have the advantage of being human-readable. However, they are primarily useful for agent platforms operating on reasonably powerful hardware and within fast networks, due to their large overheads. The bit-efficient encoding scheme is specifically designed to be simple for machines to parse and 
requires low communication bandwidth, and was thus chosen for the HARP middleware. The specifications for the FIPA Bit-Efficient encoding can be found in [97].

Next, FIPA defines how ACL messages should be used in interactions. FIPA defines a number of different message types, called Communicative Acts, which are used in interaction sequences known as Interaction Protocols. The only mandatory Communicative Act is called the not-understood message, which should be an agent's response to any message it receives but cannot understand. As long as an agent is capable of replying with a not-understood message to any message it receives, it is considered FIPA-compatible. However, to be useful, an agent should be able to understand and reply to a wider variety of message types.

An example of a useful interaction sequence, known as the query-if interaction protocol, is shown in Figure 5.4b. The sequence begins when an agent sends a query-if message type to another agent. This message type is used to make a request for information, the details of which are stored in the message body. The header of the message will contain query-if in its message type field, and will contain a unique conversation ID, which is generated by the initiating agent. Conversation IDs are used to keep track of which message belongs to which interaction, as an agent may be carrying out several interactions at once. Provided the recipient understands the message, it will reply with either an agree or refuse message type. The refuse response indicates that the recipient is not willing to proceed with the interaction, perhaps because it is currently busy, or it does not support the initiated interaction protocol. An agree response indicates that the recipient is willing to proceed with the interaction, and in this case will attempt to service the request. Once the recipient has had time to service the request, it will reply with the requested information in the body of an inform-result message, or it will reply with a failure message. Failure could be due to the requested information being private, or non-existent, amongst others.

As shown in Figure 5.3, HARP agents understand all the message types required to participate in Query and Request interaction protocols. Examples of Query interactions include querying an agent with access to temperature sensor peripherals for sensor readings, or querying the AMS for a registered agent's address. Examples of Request interactions include requesting an agent with access to a reaction wheel to provide a torque, or requesting an agent to start producing a real-time telemetry stream. While FIPA defines several other interaction protocols, these two provide a solid base from which a lot of functionality can be achieved. Further interaction protocols can be added in the future.

As mentioned, the details of a query or request are given in the message body or contents (as opposed to header). FIPA suggests implementing a content language with a well-defined syntax and semantics to aid the interaction of heterogeneous agents. Examples of such 
languages include the FIPA Semantic Language (SL), Knowledge Interchange Format (KIF) and Constraint Choice Language (CCL). However, due to the added processing power required to parse these languages and the relatively simple interactions envisioned for HARP agents, no formal content language is employed. Instead, the message contents is simply a byte array with interaction parameters stored in known indices. Implementing a formal content language is feasible in the future.

\subsection{Extended Features}

In addition to the core FIPA Abstract Architecture components, the HARP middleware includes several features which tailor it specifically to the Artificial Stem Cell Architecture. These include its support for real-time messaging, autonomous task allocation, agent redundancy, and its ability to recover from agency failures. These features are described in the subsections that follow.

\subsubsection{Real-Time Messaging}

ACL messages are used by agents to exchange information in a process known as an interaction protocol. However, since ACL messages are transported using the ISO-TP protocol, which involves reassembling messages from a number of frames, they are not well suited to real-time communication. Therefore, the ACL communication stack also makes provision for real-time CAN traffic. Messages with a cell ID of zero, which translates to CAN message IDs 0-126, are reserved for real-time frames. Since these message IDs are numerically lower than any ACL messages, which have cell IDs starting from one, they will receive priority on the CAN bus. Real-time messages are automatically forwarded by all routers, in both directions, simulating a single bus system. It is envisioned that ACL interactions will be used to set up producer/consumer relationships between protein agents, whereafter these raw, real-time message frames will be used to transfer time-sensitive data. For example, a protein agent running an attitude estimator may use FIPA ACL messages to request that another protein, interfaced to a gyroscope sensor, start to produce a stream of periodic, real-time sensor readings. 


\subsubsection{Task Allocation Entity}

A system can be defined by the list of tasks it is required to be executing at any one time. Just like in a biological organism, in a system based on the Artificial Stem Cell Architecture, it is up to the individual cells to cooperate to ensure that this list of tasks is performed. This process involves sensing information about the system state and manipulating the life cycles of proteins.

In biological multicellular systems, individual cells can gather information about the system state by measuring the concentrations of messenger molecules in the inter-cellular space. These messenger molecules are the product of protein activity and the presence or absence of a particular molecule can signify that a particular task is, or is not, currently being performed. These concentrations of messenger molecules affect the macromolecular machinery inside a cell and influence which proteins get synthesised. For example, the absence of a particular messenger molecule in inter-cellular space may invoke the macromolecular machinery to start synthesising the particular protein responsible for the missing messenger molecule. Through this feedback loop, cells are able to distribute tasks amongst themselves.

A simplified version of this process underlies the P2P task allocation strategy of the artificial cells running the HARP middleware. Within each cell, which is its own agency, a Task Allocation Entity (TAE) is responsible for interacting with other TAEs to coordinate its local allocation of tasks. This process is depicted in the flowchart of Figure 5.5.

At a user defined interval, every cell's TAE queries its local AMS for its agent directory and broadcasts a list of all locally registered agents. This process is akin to the secretion of messenger molecules in relation to active protein tasks. By listening out for these broadcasts, every TAE can build and maintain a list of all the agents, and therefore tasks, currently being executed by the system. In addition, every artificial cell contains, alongside its DNA of protein firmwares, a prioritised list of system tasks. This list is identical in every cell and is written by the system developers. The TAE routinely compares this list to the current system state and will instruct its AMS to create a new agent if a particular task is found to be missing. Because the TAE process is happening concurrently on every cell within a multicellular system, several cells may detect a missing task almost simultaneously. To minimise the instantiation of duplicate agents, each cell is given a unique priority within the system. The TAE will immediately request its AMS to retire an agent if it is detected to already be executing on a higher priority cell. In a biological cell, the role of the prioritised task list is encoded in the transcription factors and DNA in the form of enhancer and promoter sequences. 


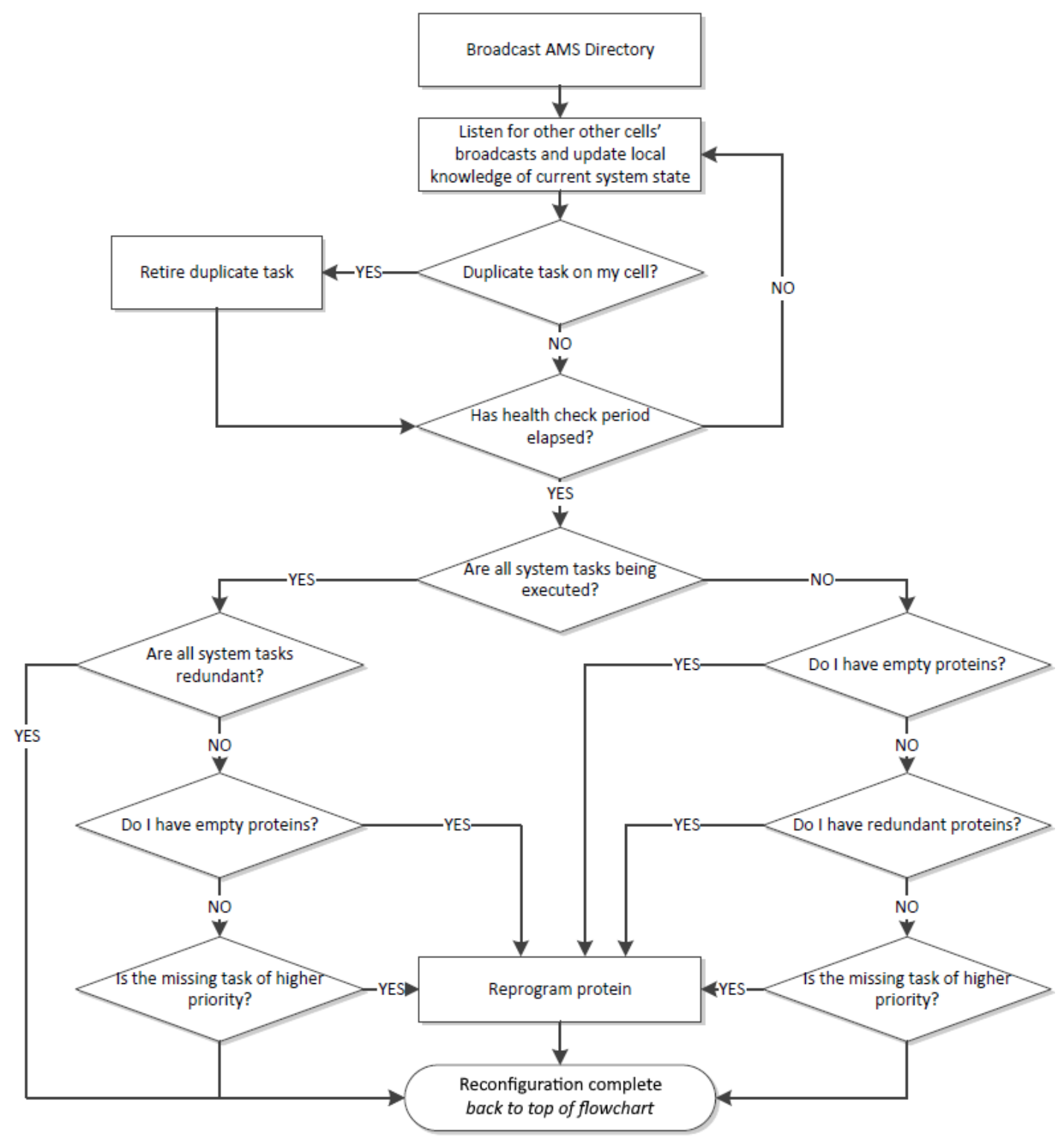

Figure 5.5: Flowchart depicting the task allocation strategy executed concurrently and asyncronously by every cell's Task Allocation Entity. 
If a cell's TAE discovers that a high priority task is not currently executing, but has no free proteins on which to deploy the relevant agent, it will request its AMS to replace a lower priority agent with the high priority one. However, this will only occur after a defined waiting period, during which other cells, with potentially free proteins, also have a chance to detect and act on the missing task. This process is not globally optimum, as the protein that is replaced may not be the lowest priority globally. Instead, it will be the lowest priority on its particular cell. However, the replacement will trigger a series of replacements throughout the system, ending with globally highest set of tasks being executed. While not optimally efficient, this process is robust as no negotiation is required between cells. In addition, protein replacements are expected to occur infrequently and only in response to failures or significant environmental changes.

\subsubsection{Redundancy}

To hasten system recovery after a failure, redundant agents may be introduced. Both cold and hot redundancy is supported. Cold redundant agents are proteins which have been programmed with the relevent agent firmware, but are physically switched off. Activation of a cold redundant agent proceeds through boot-up, AMS registration and runtime variable update processes, before taking over from the failed agent. On the other hand, hot redundant agents are powered on and pre-registered, but are in a paused state. Runtime variable updates may still occur to keep the hot redundant agent in sync with its active counterpart. The activation of either form of redundancy takes less time than it takes to program a new protein.

If redundancy is enabled, the TAE entity will attempt to fill unused proteins with redundant agents, again starting from the highest priority. Redundant agents are always replaced before any active agent in the event of a task reshuffle.

\subsubsection{Agency Services Failure}

In a typical agent platform, the agency presents a potential single point of failure. As explained, the agency is responsible for providing a number of services. These include both mandatory services, such as directory lookups, message routing and agent lifecycle management, and HARP specific services, such as task distribution and runtime variable management. Without these services, the multicellular system would fail. Thus, in order to remove this potential single point of failure, the HARP agencies are, themselves, mobile. 
As explained in Section 4.3.2, failure of the acting macromolecular machinery protein (and therefore the agency) results in a recovery procedure akin to artificial mitosis. This process is orchestrated by the HARP middleware.

Upon failure of the MM protein, the protein health-check service ceases. This is detected by a hardware watchdog timer on the cell which activates a hardware bridge between the programming and inter-protein buses of the stricken cell with those of its neighbouring cell. At this point the neighbouring cell's agency will detect the additional proteins which have now been added under its control. In effect, the neighbouring agency's AEE has expanded. Following the same process as reprogramming one of its proteins with a new agent, the agency will reprogram one of the new proteins (which are physically located on the stricken cell) with the agency firmware. Once that protein boots, it becomes the active agency of the previously stricken cell. The programming buses are disconnected and the two cells operate independently once again. The new agency will attempt to revive the old, stricken MM protein for reuse as a standard agent protein.

Through this process agencies are kept functional above all else, despite increasing hardware failures.

\subsection{Summary}

This chapter described the Hybrid Agent Real-Time Platform, which is a set of middleware designed to facilitate communication and cooperation between the artificial cells of Chapter 3. Through the implementation of a decentralised task management strategy, the HARP middleware allows a multicellular system to gracefully degrade in response to hardware failures.

The HARP middleware turns each artificial cell into an agent platform based on the Foundation for Intelligent Physical Systems Abstract Architecture. Each agency implements the compulsory FIPA components, including an Agent Management System, Agent Communication Channel, and Agent Execution Environment. Furthermore, the HARP communication stack implements the FIPA specifications for Agent Communication Language, encoding, communicative actions, and interaction protocols.

Unlike the majority of agent platforms, designed for desktop computers, the HARP middleware is expected to run on the resource constrained, embedded environment of a low-cost satellite. This necessitates a small memory footprint, fault tolerance, and support for real-time 
operations. To fulfil these requirements, the HARP middleware distinguishes itself from typical agent platforms in several areas.

Firstly, the HARP middleware does not make use of virtual machines or interpreters and instead uses discrete processors as its agent execution environment. This has the advantage of reducing the agency memory footprint and enabling protein agents to perform real-time tasks.

Secondly, in place of resource-intensive protocols such as HTTP and IIOP, the HARP communication stack makes use of ISO-TP over CAN 2.0A. This design saves memory and processing time, as well as allowing real-time CAN traffic to share the network and be given priority over ACL communication.

Third, the HARP middleware embeds a peer-to-peer task manager in each agency, allowing systems composed of Satellite Stem Cells to autonomously reconfigure in the event of failures. The task manager supports the allocation of redundant protein agents, to reduce recovery time, and preserves user-defined runtime variables using a form of 'soft' agent mobility.

Finally, while most agent platforms can recover from the failure of an agent, the HARP middleware can additionally recover from a complete agency failure. This is possible because agents and agencies execute on the same discrete processors within an artificial cell. Thus, through a process akin to cell mitosis, the agency can be moved from a failed processor to a functional one, possibly displacing an agent protein in the process. This capability prevents agencies from becoming a potential single point of failure. 



\section{Chapter 6}

\section{Reliability Analysis and Simulation}

In this chapter, the theoretical reliability of multicellular systems is investigated. First, the derivation of an analytical solution for predicting the lifetimes of simple multicellular systems is presented. Using this solution, the reliability of multicellular systems is compared against systems featuring traditional forms of redundancy. Thereafter, the design of a MATLABbased, multicellular system simulator is presented. The simulator is used to verify the analytical reliability predictions. Additionally, it is used to study more complex multicellular systems, including those with peripherals. The results of the analytical and simulationbased analyses are formulated into a set of guidelines for the configuration of multicellular systems.

Because the Satellite Stem Cell Architecture is primarily concerned with extending the overall system lifetime, it allows a level of system performance degradation over time. Thus, throughout this chapter, only permanent failures are considered, and transient failures, such as radiation induced single event transients, and parametric failures, such as a device drawing more current than expected, are not modelled. The failure rates used throughout this chapter are for demonstration and comparison purposes only, and are not necessarily representative of any particular real-life system.

Section 6.1: Introduction To Reliability Analysis, and Section 6.2.1: Common System Architectures, are based on well-known reliability equations described in [19]. All further derivations, where not specifically referenced, are original. 


\subsection{Introduction to Analytical Reliability Analysis}

Every device, without external maintenance, will eventually fail. The time to failure is the duration of time the device was functioning properly before failure occurred. Time to failure is a value of the random variable $T$ and is a real number in the interval $[0, \infty)$. Since $T$ is a random variable, it can be described by a cumulative distribution function, as given by Equation 6.1.

$$
F(t)=P(T \leqslant t), \quad t \geqslant 0
$$

$F(t)$ represents the probability that the system will fail by time $t$, and is usually known as the unreliability function. Similarly, the reliability function $R(t)$ is defined by Equation 6.2 .

$$
R(t)=P(T>t)=1-F_{T}(t)
$$

Thus, reliability is the probability of no failures occurring in the time period $[0, t]$.

Often, the random variable $T$ will be described by its probability density function, $f(t)$. The reliability function, $R(t)$, can then be calculated as given by Equation 6.3. If the failure probability density function of a system is known, the probability of the system being functional at a certain point in time can be calculated by substituting for $t$.

$$
R(t)=1-\int_{0}^{t} f(x) d x
$$

Related to the reliability function is the failure rate function. It gives the expected number of failures per unit time at a given instance in time. The failure rate function $h(t)$ can be calculated using Equation 6.4

$$
h(t)=\frac{f(t)}{R(t)}
$$

The simplest failure rate function is a constant failure rate, which implies that failure is a random event and is equally likely to occur at any moment in time. A constant failure rate is a common simplifying assumption for systems whose failure probability density function is not well understood. The probability density function of a system with a constant failure rate 

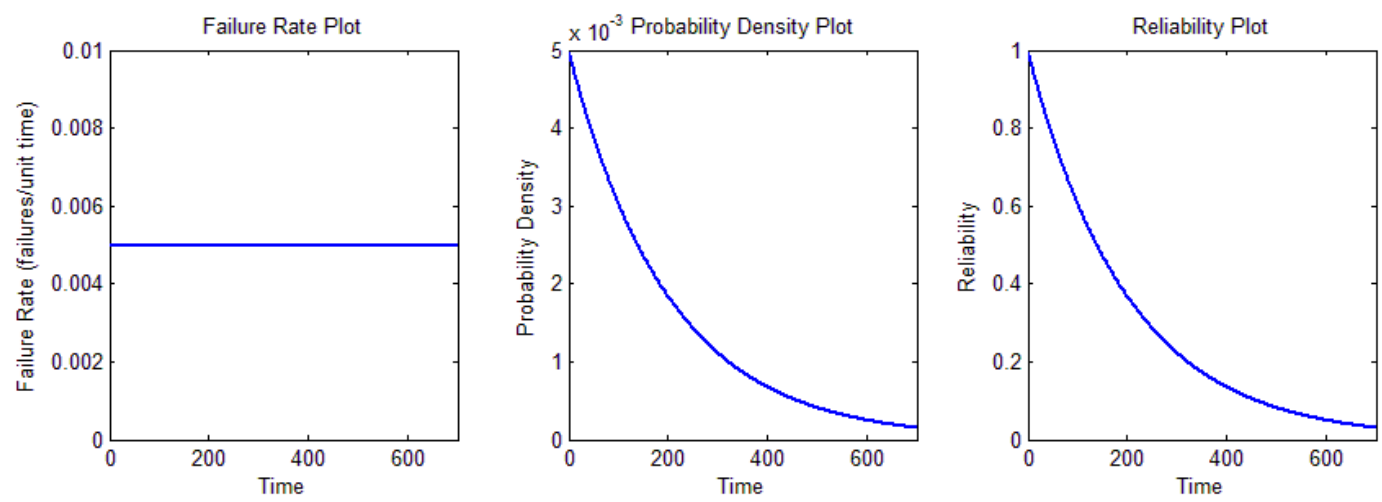

Figure 6.1: Failure rate, probability density and reliability plots of a system known to suffer from continuous random failures.

is given by Equation 6.5. It involves only a single parameter, $\lambda$, which is known as the 'Mean Time to Failure' (MTTF). The MTTF of a system with a constant failure rate represents the expected time after which $63.2 \%$ of such systems are expected to have failed.

The failure rate, probability density and reliability functions of a system with a constant failure rate are shown in Figure 6.1.

$$
f_{\text {const }}(t)=\lambda e^{-\lambda t}
$$

Usually, failure rates are not constant. Without maintenance, most systems suffer from an increasing failure rate over time, as components begin to wear out. In contrast, newly designed systems can suffer from infant mortality, which is characterised by a high initial failure rate which decreases over time. These behaviours can be modelled with a Weibull Distribution, which is commonly used in reliability analysis. It has two parameters, called the shape parameter $\beta$ and the scale parameter $\theta$. With an appropriate choice of the shape parameter the Weibull distribution can model increasing failure rates (wear-out), decreasing failure rates (infant mortality) and constant failure rates. The Weibull probability density function is given in Equation 6.6, and a Weibull distributed reliability function has the form given in Equation 6.7.

$$
\begin{gathered}
f(t)=\frac{\beta}{\theta}\left(\frac{t}{\theta}\right)^{\beta-1} e^{-\left(\frac{t}{\theta}\right)^{t}} \quad t \geqslant 0 \\
R(t)=e^{-\left(\frac{t}{\theta}\right)^{\beta}}, \quad \theta>0, \beta>0, t \geqslant 0
\end{gathered}
$$



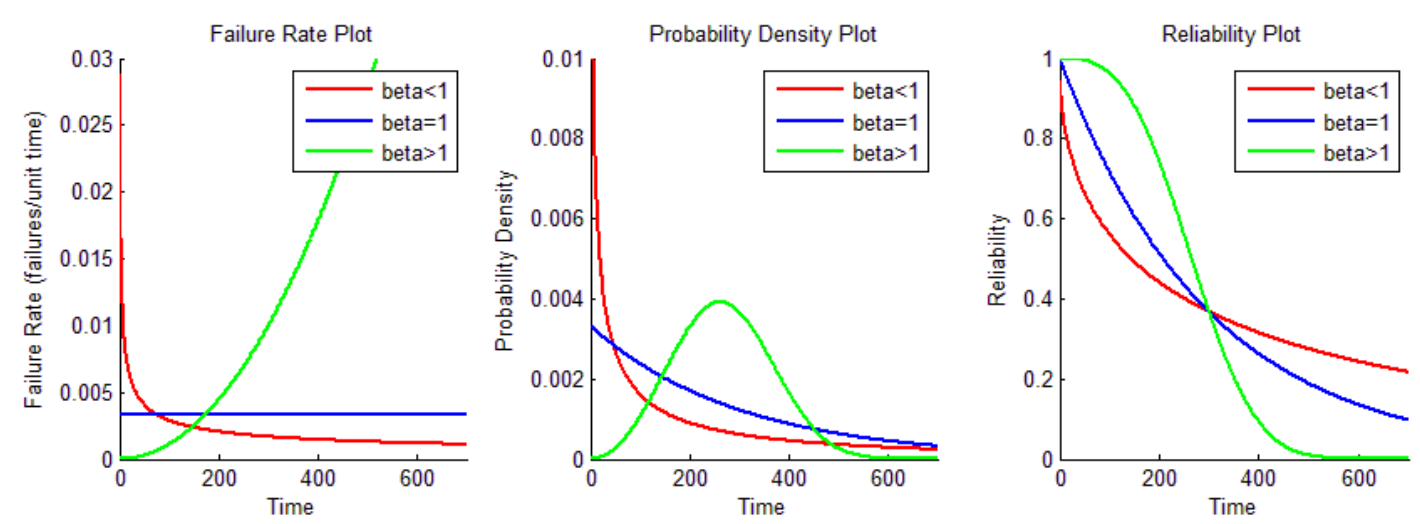

Figure 6.2: The effect of the Weibull Distribution scale parameter on failure rate, probability density and reliability plots

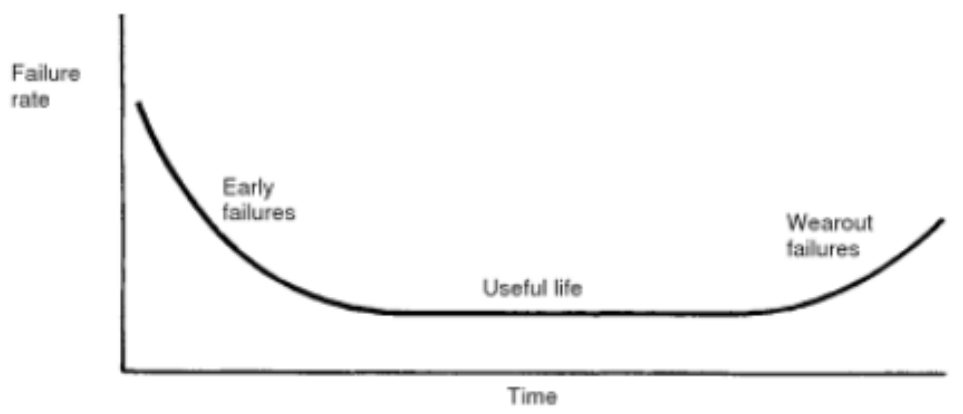

Figure 6.3: The commonly-seen bathtub reliability curve [98].

The shape parameter $\beta$ is dimensionless and the scale parameter $\theta$ is expressed in units of time. The effect of different scale parameters can be seen in Figure 6.2. A decreasing failure rate is modelled with $0<\beta<1$, a constant failure rate is modelled with $\beta=1$ and an increasing failure rate is modelled with $\beta>1$.

The 'bathtub curve' is a commonly-seen failure rate function which combines the three forms of Weibull functions into one [98]. It denotes a system which initially suffers from infant mortality (decreasing failure rates), followed by a period characterised by a relatively constant failure rate, before suffering from an increasing failure rate due to wear-out. A bathtub curve failure rate function is shown in Figure 6.3.

The MTTF of a system with a non-constant failure rate can be calculated as the expected value of the system's reliability function. This is shown in Equation 6.8.

$$
M T T F=\int_{0}^{\infty} R(t) d t
$$


However, when the only reliability information on a system is specified as an MTTF, a constant failure rate must be assumed if any reliability analysis is to be performed.

\subsection{Reliability Equation Derivation}

An analytical reliability function for a system based on the Satellite Stem Cell Architecture is needed for two reasons. Firstly, an analytical solution gives insight into the behaviour of the system and will aid designers in determining the optimal ratios of proteins per cell and cells per system, based on high-level reliability requirements. Secondly, provided the analytical solution is not too computationally intensive, there is potential for a multicellular system to continuously predict its own reliability and react through autonomous reconfiguration. For example, a multicellular satellite, upon receiving indication of an upcoming solar flare, may reconfigure from a high performance to high reliability state. It could do so by repurposing low priority proteins into spares for high priority proteins. Monte Carlo simulations (which can also be used to approximate a system's reliability function) can be used to verify design decisions using the multicellular system simulator, but are too inefficient to be run on resource-constrained embedded systems.

The following analyses assume that all components begin to age at the same time and continue to age whether they are power-on or not. This assumption is consistent with several on-orbit failure modes, including failure from total ionising dose, thermal cycling stress, and charge build up. In addition, the analyses assume perfect failure coverage. The analysis begins with a purely computational system, meaning that there are no attached peripherals and every protein has the potential to run any task. Thereafter, in Section 6.5, the effect of attached peripherals is investigated.

\subsubsection{Common System Architectures}

As a starting point in the derivation of a reliability equation for multicellular systems, it is worth briefly going over some common system architectures. Comparisons between the reliabilities of different system architectures will be given once the multicellular system reliability equation has been derived (Section 6.3). The simplest system architecture is a pure series system, which is also known as a single-string system. This architecture is depicted in Figure 6.4. In the simplest case, such a system could be modelled using only the individual reliabilities of the subsystems themselves. However, in practical systems the subsystems 
are typically supported by common infrastructure such as power and communication buses. Therefore, two additional failure models related to shared infrastructure are included. The common component model encapsulates all the failure modes which are independent of the number of subsystems. For example, failure of the power supply. On the other hand, the support component model encapsulates failure modes which are dependent on the number of subsystems. For example, a lock-up of the communications bus becomes more likely the more subsystems are added. These additional failure models will become more important once multicellular architectures are examined.

The reliability function for a single string system as depicted in Figure 6.4 is given by Equation 6.9. $R_{c}, R_{s}, R_{p}$ are the reliabilities of the common, support, and subsystem models respectively, at a particular moment in time, as given by each model's reliability function. In other words, $R$ should be written as $R(t)$, but has been abbreviated in the following sections for clarity. The number of subsystems is denoted by $n$. In a single string system, all the subsystems are required to be functional in order for the system to be functional.

$$
R_{\text {string }}=R_{c}\left(R_{S}\right)^{n} \prod_{i=1}^{n}\left(R_{p i}\right)
$$

The simplest form of redundancy involves duplicating the single-string system into what can be called a multi-string system. This includes the duplication of the supporting infrastructure, as shown in Figure 6.5. The reliability for such an architecture is given by Equation 6.10. $R_{\text {string }}$ is the reliability of a single string at any moment in time, as calculated using Equation 6.9. The number of strings in the system is denoted by $m$.

$$
R_{\text {mstring }}=1-\left(1-R_{\text {string }}\right)^{m}
$$

An improvement to the multi-string architecture involves 'cross-strapping' components, as shown in Figure 6.6. Cross-strapping involves interconnecting components of different strings so that a functional system is possible even if unique failures have occurred in each string. In practice, cross-strapping is difficult to implement and can lead to excessive amounts of harnessing and mass. In addition, the strain on the support components, such as communication buses, is multiplied by the number of strings in the system, as each support component must now be able to service components in any string. Given $F_{c}=1-R_{c}, F_{S}=$ $1-R_{s}^{m n}$ and $F_{p}=1-R_{p}$, the reliability of a cross-strapped, multi-string system is given by Equation 6.11. 


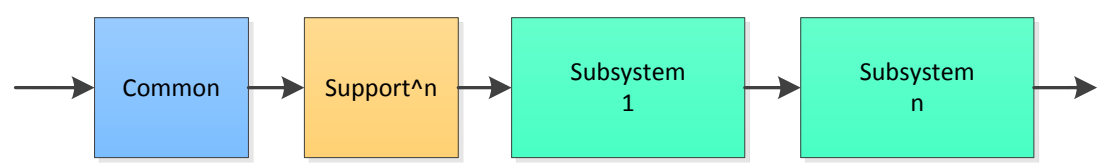

Figure 6.4: Schematic representation of a single-string system architecture.

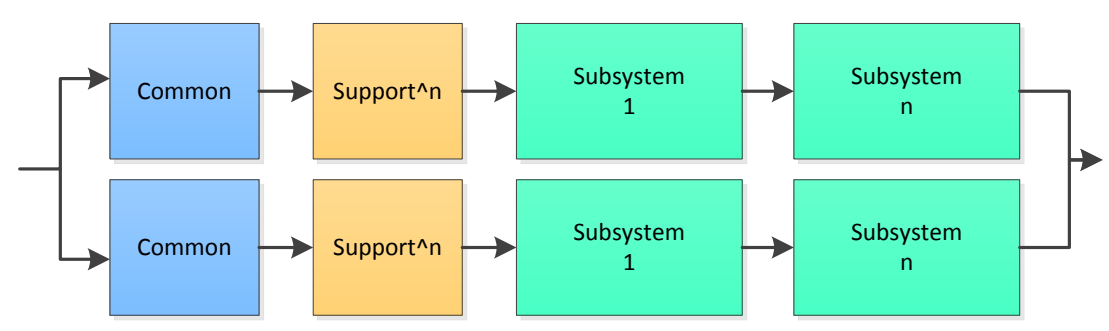

Figure 6.5: Schematic representation of a multi-string system architecture.

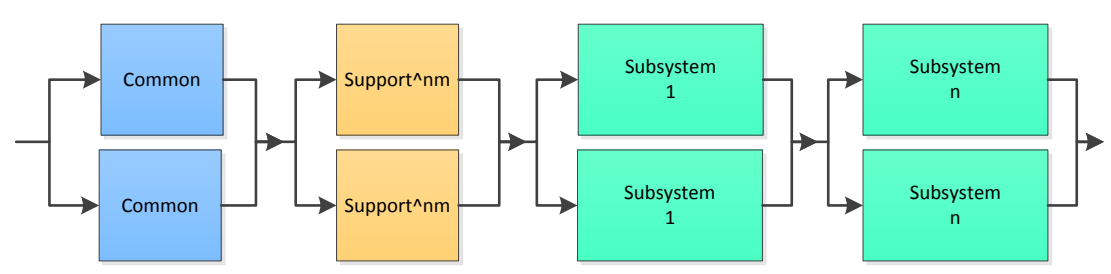

Figure 6.6: Schematic representation of a cross-strapped, multi-string system architecture.

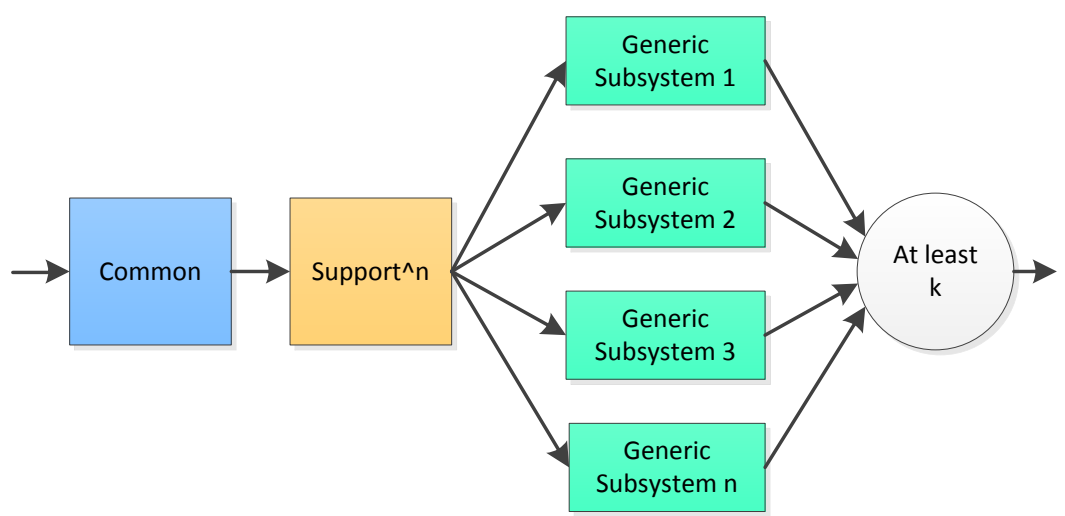

Figure 6.7: Schematic representation of an n-choose-k system architecture. 


$$
R_{c s t r a p}=\left(1-F_{c}^{m}\right)\left(1-F_{s}^{m}\right) \prod_{i=1}^{n}\left(1-F_{p i}^{m}\right)
$$

The final architecture to cover before moving onto multicellular systems is the k-out-of-n architecture. In this architecture, every subsystem is generic and has the ability to perform the role of any other subsystem in the group. Thus, as long as a sufficient number of these generic subsystems are functional, all the required systems tasks can be performed. The set of subsystems is typically called a pool, and the ratio of number of subsystems in the pool to minimum number of required subsystems defines the system's redundancy. In an ideal k-out-of-n system, all components of the system would reside in the pool. However, in practice, as with the other system architectures, the generic subsystems typically require additional supporting infrastructure. Thus, a k-out-of-n architecture can be modelled as shown in Figure 6.7. It should be apparent that the supporting infrastructure presents a potential weak point in an otherwise very reliable architecture. The reliability equation for such a system is given by Equation 6.12, where $n$ is the total number of generic subsystems in the pool, and $k$ is the minimum number required for the system to be functional.

$$
R_{\text {pool }}=R_{c} R_{s}^{n} \sum_{i=k}^{n}\left(\begin{array}{c}
n \\
i
\end{array}\right) R_{p}^{i}\left(1-R_{p}\right)^{n-i}
$$

At this point the similarity between a k-out-of-n architecture and the proposed multicellular architecture may have become apparent. Thus, the k-out-of-n architecture will serve as a starting point for the next section, in which the reliability equation of the proposed multicellular architecture will be derived.

\subsubsection{Multicellular Architectures}

In a multicellular architecture, each cell can be treated as its own k-out-of-n system. In this case, the generic subsystems are the proteins. The proteins rely on common components, such as central voltage regulators and non-volatile memory, and supporting components, including the inter-protein communication and power buses. Since a multicellular system is composed of more than one cell, there is an additional layer of supporting infrastructure between the cells of a system. This supporting infrastructure includes power and communication buses, which are assumed to decrease in reliability as more cells are added to the system. Inter-cell, common-mode failures are akin to common-mode failures between units of traditional redundancy schemes. Therefore, for comparison purposes, such failures are not 


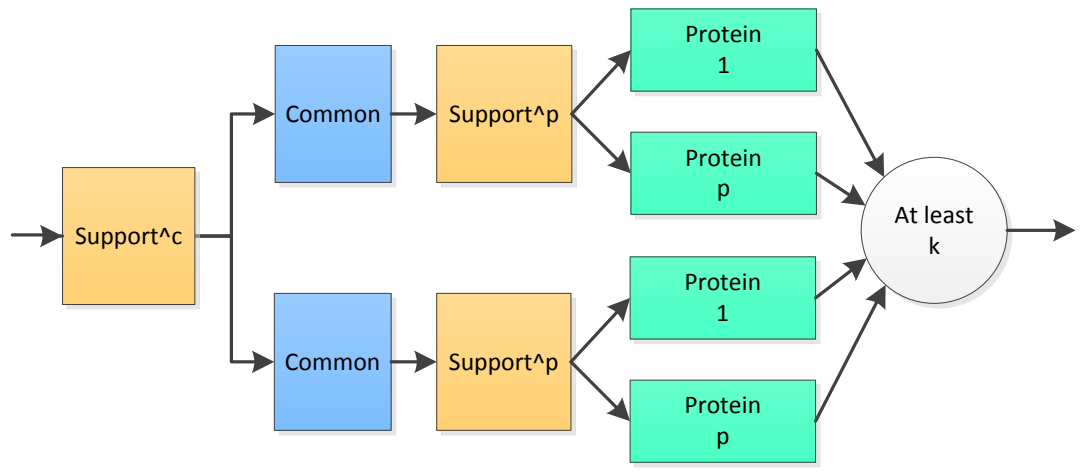

Figure 6.8: Schematic representation of a simple multicellular system composed of two cells of two proteins each.

considered. However, a common way to mitigate against common mode failures is to have two separate unit designs in the system. This could equally be applied to a multicellular system by including at least two different cell designs, based on the same specifications, in the system.

A simple multicellular system composed of two cells of two proteins each is depicted in Figure 6.8. In this case, the system is functional if sufficient proteins, across all cells, are functional. Determining the reliability of such a system is complex as the same system state can be achieved through multiple configurations. For example, referring to Figure 6.8 , a system state consisting of two functional proteins can be achieved by having a single functional cell with two functional proteins, or by two functional cells each containing only a single functional protein. In either case the system has two functional proteins, but the state consisting of only a single cell is more reliable as fewer common and supporting components are required. Therefore, all possible configurations resulting in a particular state must be considered when calculating reliability.

Since the state of a multicellular system as a whole is a function of its individual cell states, in makes sense to consider each cell on its own first. While it is common to assume only two states in reliability calculations, namely functional and non-functional, in this case a cell is said to be in one of multiple states [99]. The state represents the number of functional cells proteins that the cell can contribute to the system. At any point in time a cells state can be described using a discrete probability density function (PDF). Initially ignoring the need for a macromolecular machinery protein, the probability of being in a specific state is equal to the probability of the common and supporting components being functional, and of that specific number of proteins being functional. State zero occurs when either all of the proteins on the cell have failed, or the common or supporting components have failed. 


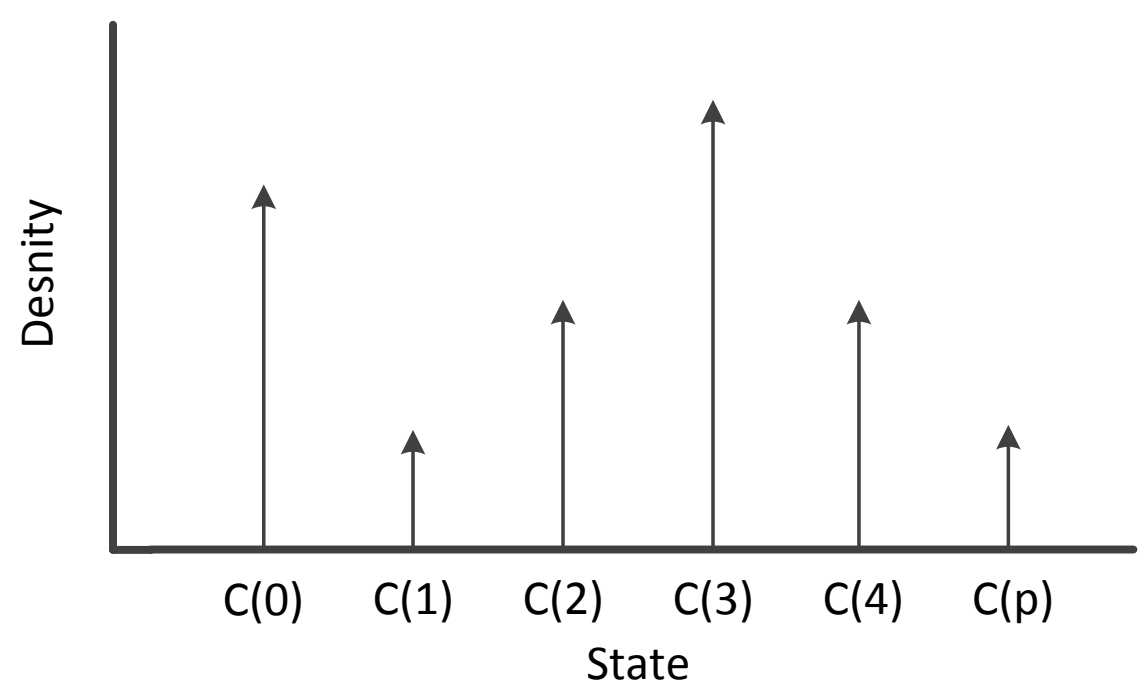

Figure 6.9: An example of a cell's discrete state probability density function, which reflects the probability of a specific number of proteins being functional at a given time.

For a cell containing $p$ proteins, the probability of exactly $k$ being functional, and therefore of the cell being in state $k$, is given by Equation 6.13. This is the same equation which underlies the n-choose-k system reliability function. In that case, the probabilities of exactly $k$, or more than $k$, were added together to determine the system reliability. Equation 6.13 is only valid for states $k>0$. The probability of being in state zero is given by Equation 6.14 .

$$
\begin{gathered}
\left.C(k)\right|_{k=1: p}=\left(R_{c} R_{s}^{p}\right)\left(\begin{array}{c}
p \\
k
\end{array}\right) R_{p}^{k}\left(1-R_{p}\right)^{p-k} \\
C(0)=\left(1-R_{c} R_{s}^{p}\right)+\left(R_{c} R_{s}^{p}\right)\left(\begin{array}{c}
p \\
0
\end{array}\right) R_{p}^{0}\left(1-R_{p}\right)^{p-0}
\end{gathered}
$$

Using Equations 6.13 and 6.13, a cell's discrete state PDF, $C(k)$, can be constructed as shown in Figure 6.9.

Now, given a system of $c$ cells, the system's state pdf can be calculated through the convolution of the individual cell pdfs $C_{i}(k)$. This process is expressed in Equation 6.15.

$$
S_{\text {proteins }}(k)=C_{1}(k) * C_{2}(k) * C_{3}(k) \ldots * C_{c}(k)
$$

Once again the system state, $k$, represents the number of functional proteins. More specifically, it represents the total number of functional proteins across all cells. States with a sufficient 
number of proteins will result in a functional system, while those states with too few translate to a non-functional system. Thus, system reliability can be calculated by summing the probabilities of all functional states. This is shown in Equation 6.16. The additional term, $R_{s}^{c}$, models the reliability of the supporting infrastructure between cells, which is also required to be functional in order for the system to be functional.

$$
R_{\text {multicell }}=R_{s}^{c} \sum_{k=n}^{c p} S(k)
$$

Up until now, the need for macromolecular machinery (MM) proteins has been ignored. However, to more accurately predict the reliability of a multicellular system, the overheads of the MM proteins need to be taken into account.

One each cell, the first functional protein is reserved for the MM. Therefore, cells need to have at least two functional proteins in order to contribute to the system state. This is modelled by essentially reducing the cell state by one. In addition, the probability for a cell to be in state zero is increased, as state zero now occurs when the cells supporting infrastructure has failed, or the cell has zero or one functional proteins. Equations 6.13 and 6.14 are updated to reflect these changes, resulting in the updated Equations 6.17 and 6.18. These can be used as before with Equations 6.15 and 6.16 to calculate a multicellular system's reliability.

$$
\begin{aligned}
& \left.C(k)\right|_{k=1: p}=\left(R_{c} R_{s}^{p}\right)\left(\begin{array}{c}
p \\
k+1
\end{array}\right) R_{p}^{k+1}\left(1-R_{p}\right)^{p-(k+1)} \\
& C(0)=\left(1-R_{c} R_{s}^{p}\right)+\left(R_{c} R_{s}^{p}\right) \sum_{i=0}^{1}\left(\begin{array}{c}
p \\
i
\end{array}\right) R_{p}^{i}\left(1-R_{p}\right)^{p-i}
\end{aligned}
$$

Figure 6.10 presents a first look at the reliability functions, as calculated using Equation 6.16, of several example multicellular systems. Each system is composed of 120 proteins, but distributed amongst a differing number of cells. Each system is said to have 24 critical tasks, and therefore requires at least 24 of its 120 proteins to be functional. Constant failure rates are assumed for proteins (MTTF of 5 years), common components (MTTF of 10 years), and supporting components (MTTF of 1000 years). These values are chosen purely for demonstration purposes.

From Figure 6.10 it becomes apparent that choosing the most reliable configuration of proteins is not trivial. Different systems, with different configurations of proteins, present the highest reliability at different mission phases. Similarly, if the hazard functions are plotted, 


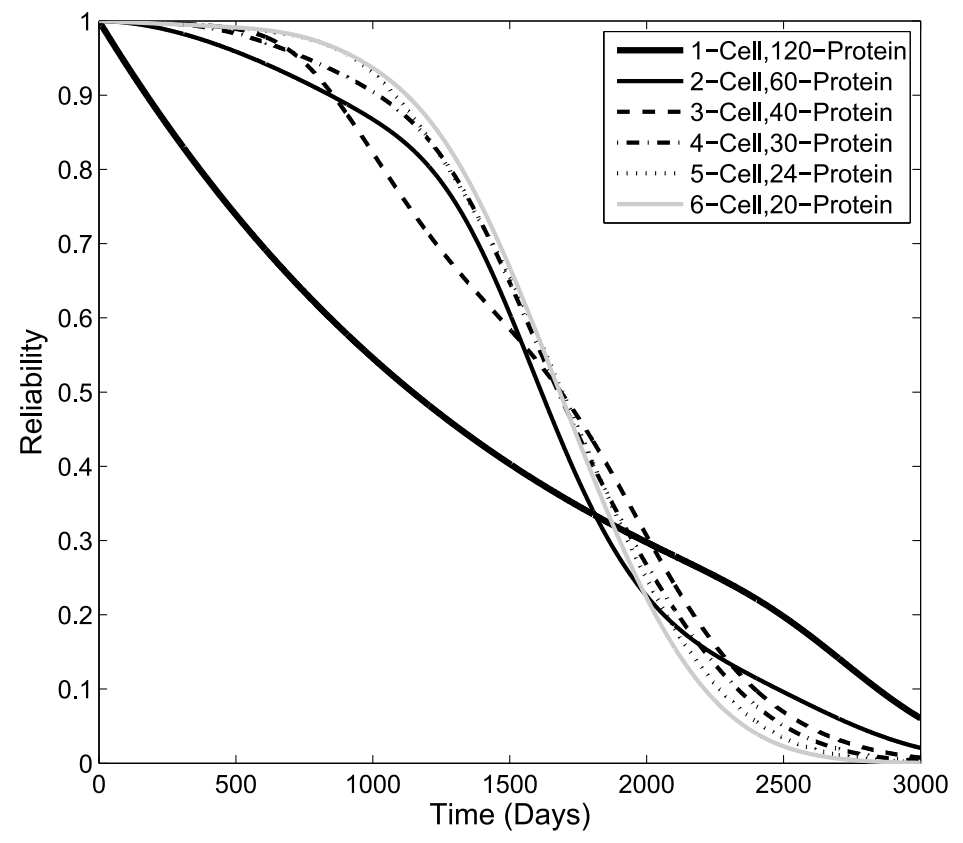

Figure 6.10: Comparison of the reliability functions of different 24-task multicellular system configurations.

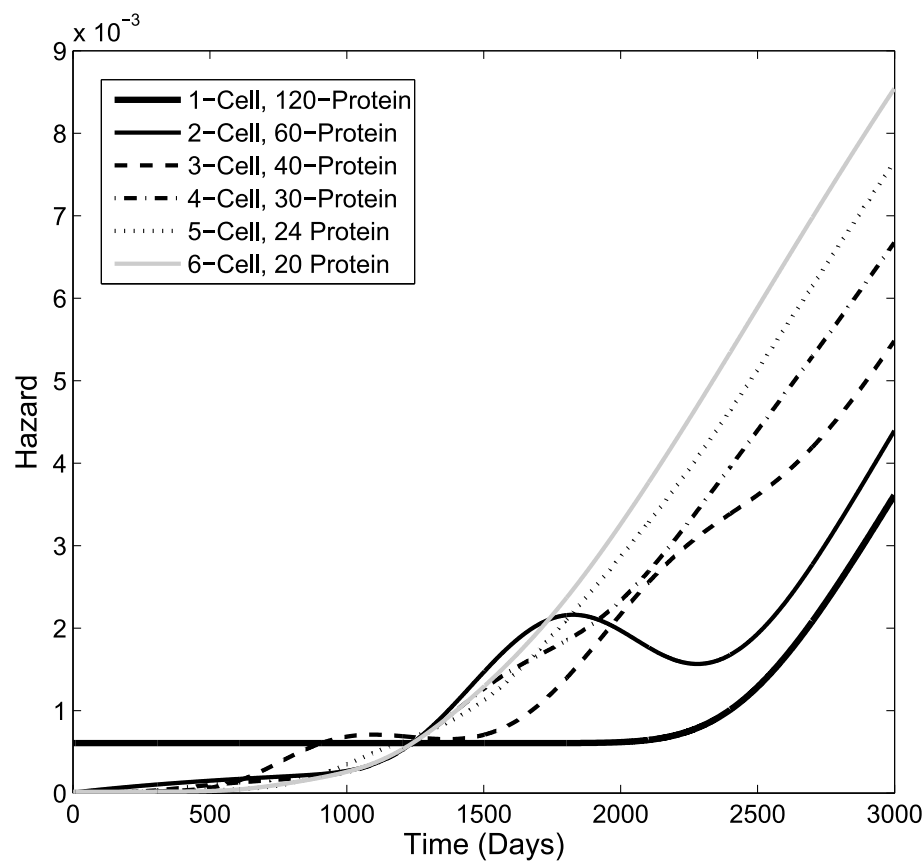

Figure 6.11: Comparison of the hazard functions of different 24-task multicellular system confgurations. 
as shown in Figure 6.11, undulating failure rates are seen. This appears counter-intuitive, given that all of the components in the system were given constant failure rates.

To gain a better understanding of the system behaviour predicted by the reliability functions derived in this section, the system state PDF is examined in more detail.

\subsubsection{Understanding the System State PDF}

In this section, the relationship of the system state PDF to various parameters is examined. The parameters include: mission time, number of cells, proteins per cell, and the reliability of supporting infrastructure.

Using Equation 6.15, the system state PDF can be plotted for various times within the system's lifetime. As shown in Figure 6.12, the system state PDF changes over time (Note that the PDFs are shown as being continuous for clarity, but are actually discrete). As it progresses, time has the effect of moving the expected value towards zero. This is intuitive, as the system state represents the number of functional proteins and the proteins are gradually expected to fail over time.

Figure 6.13 shows the system state PDFs of three different multicellular systems at a particular moment in time. The three systems are composed of one, two, and three cells respectively (each cell contains an identical number of proteins, so the three systems have differing numbers of total proteins). From Figure 6.13, it is apparent that, ignoring the peak at zero, the number of peaks in the PDF is the same as the number of cells in the system. Intuitively, the peaks can be explained by remembering that the system state is determined by both the number of functional cells and the number of functional proteins per cell. Knowing the number of functional cells in a system at any one time gives a lot of information about the likely total number of functional proteins in the system at that time. Essentially, each number of functional cells comes with its own state PDF which shows up as a peak in the full system state PDF.

Mathematically, the peaks can be explained by revisiting Figure 6.9, which shows an example discrete protein state distribution of an individual cell. If the distribution is pictured as being continuous instead of being discrete, a cell's protein state distribution can have one or two peaks. One peak occurs at the the most likely number of functional proteins. A second peak can occur at $C(0)$, which represents the probability of cell failure due to failed common or supporting components. As explained in Section 6.2.2, the final system state PDF is calculated through the convolution of the individual cell PDFs. Convoluting two 


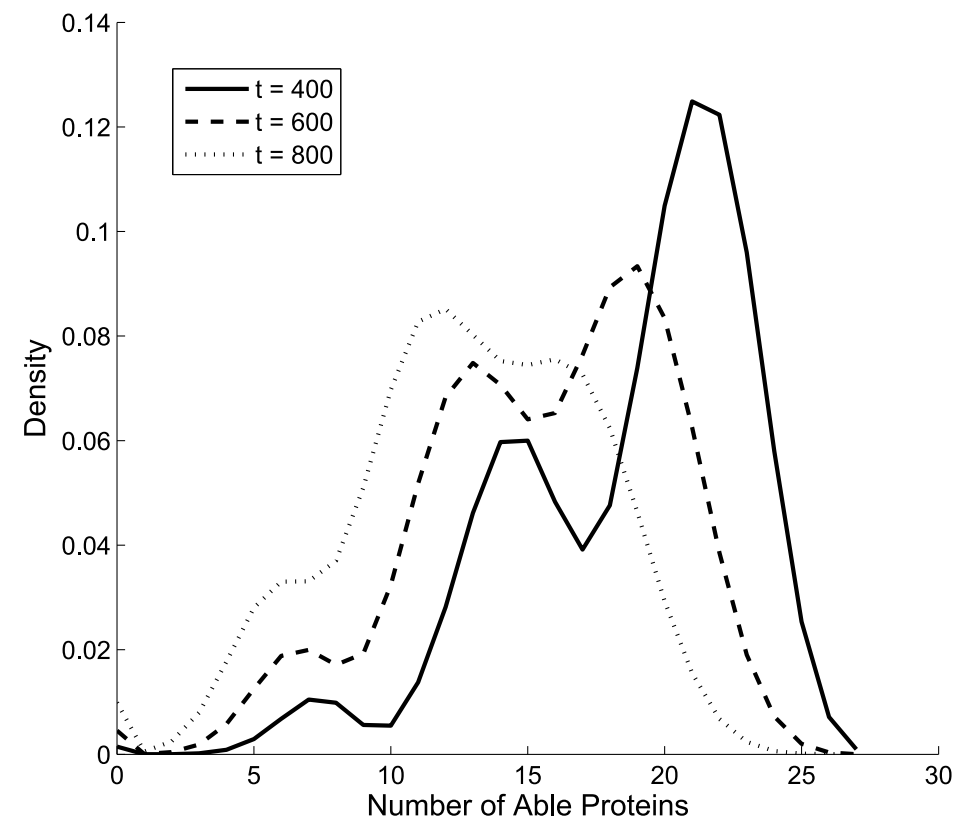

Figure 6.12: The system state PDF changes over time. It is shown here as continuous for clarity, but is actually discrete. Able proteins are those which are functional and not reserved for MM duty.

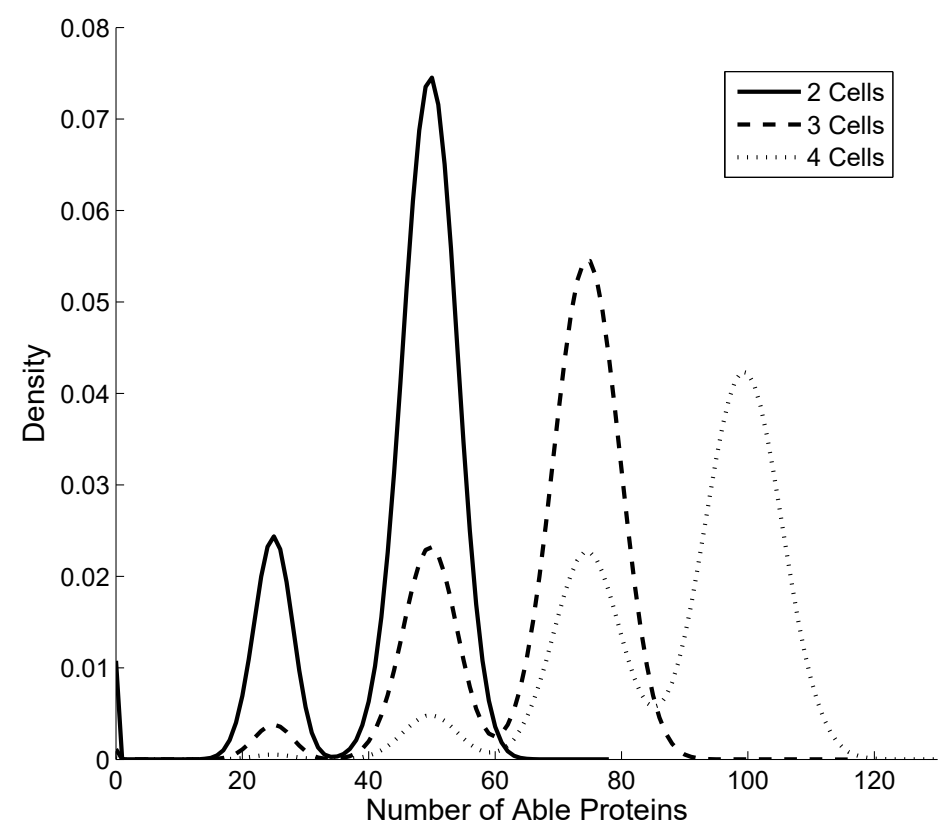

Figure 6.13: The effect of number of cells on the system state PDF. Excluding the peak at zero, the number of peaks is equal to the number of cells in the configuration. 


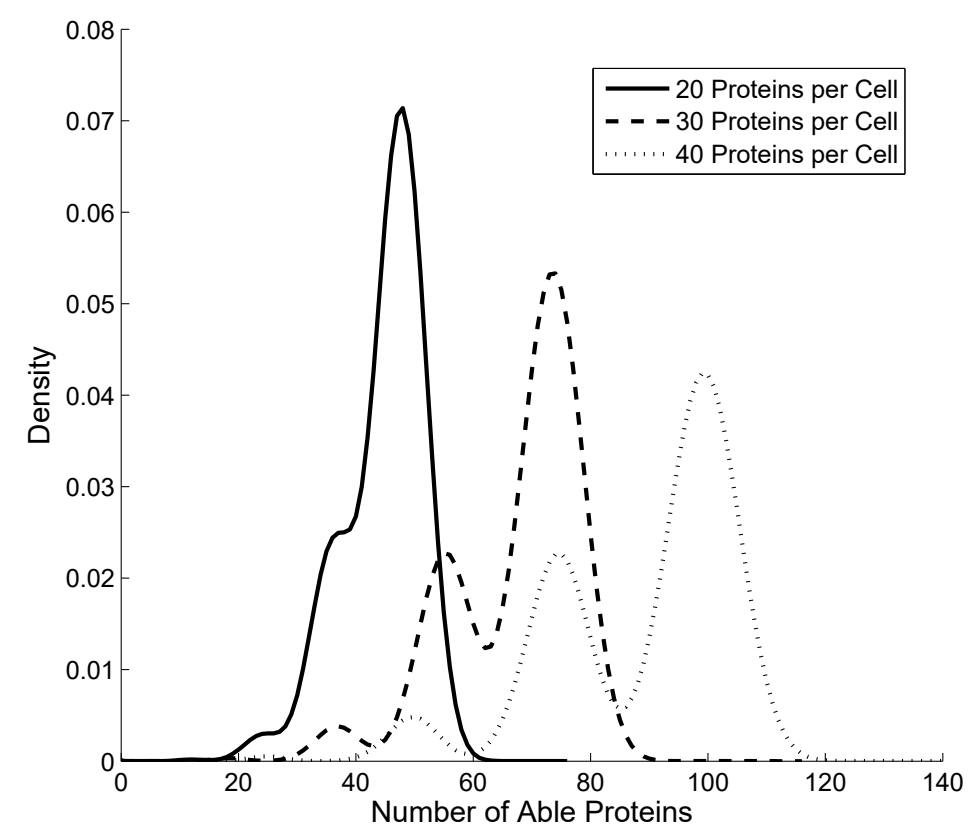

Figure 6.14: The effect of proteins per cell on the system state PDF. As the number of proteins per cell increases, the peaks move further apart and become more defined.

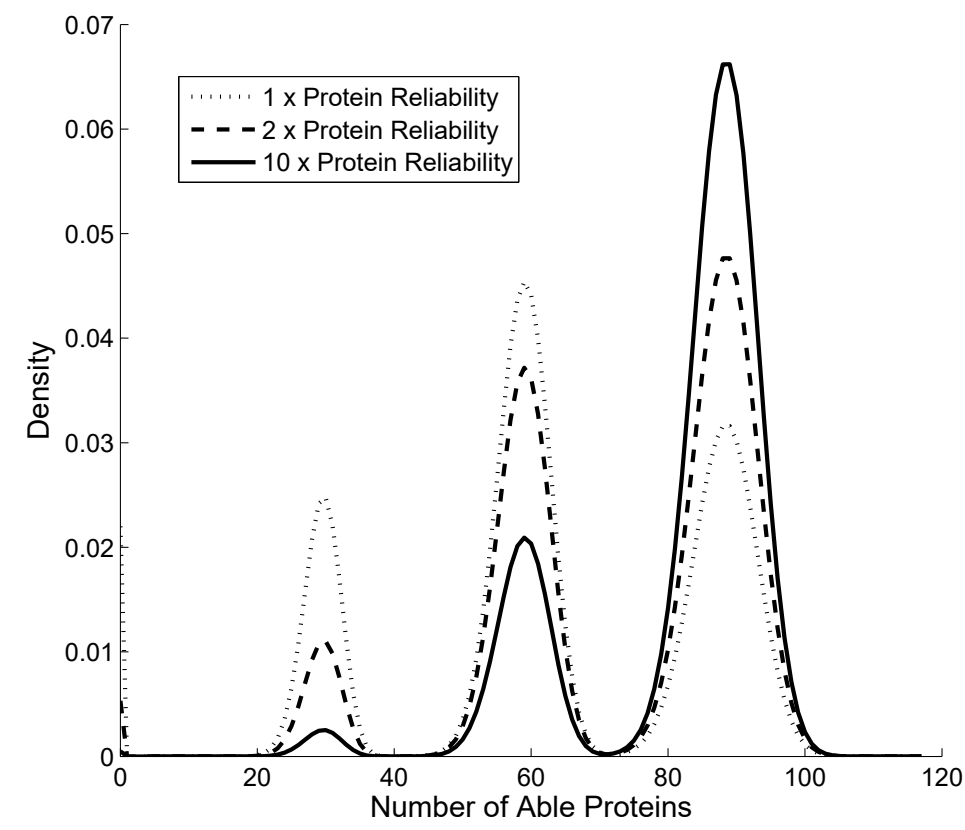

Figure 6.15: The effect of common component reliability on the system state PDF. Common component reliability affects the height of the peaks, but not their location. As common component reliability increases the distribution is shifted to the later peaks. 
single-peaked distributions would result in only one peak, but it is through the convolution of the multi-modal cell state distributions that the multiple peaks of the system state PDF appear.

In addition to number of cells, the number of proteins per cell also has a predictable effect on the system state PDF. As seen in Figure 6.14, the number of proteins per cell affects the spacing between peaks. More proteins per cell result in more clearly differentiated peaks, while in systems with relatively few proteins per cell, the peaks become almost indistinguishable.

Finally, as seen in Figure 6.15, the reliability of a cell's supporting infrastructure affects the height distribution of the peaks of the system state PDF. More reliable infrastructure shifts the height distribution towards the later peaks, while unreliable infrastructure favours the earlier peaks. The passing of time has a similar effect of shifting the expected value towards zero, but, unlike the reliability of infrastructure parameter, the passing of time additionally shifts the locations of the peaks towards the left.

With an understanding of the effects that these parameters have on the system state PDF, the multicellular system reliability function can be re-examined with more insight.

\subsection{System Reliability Sensitivity Analysis}

This section presents a sensitivity analysis based on, amongst others, the parameters examined in Section 6.2.3, and compares the reliability of multicellular architectures to more traditional multi-string and cross-strapped architectures.

Firstly, sensitivity to total number of tasks is examined. Figure 6.16 shows the reliability plots of various multicellular systems, a multi-string system, and a cross-strapped system. Each system is responsible for six tasks, which are performed by proteins in the multicellular architectures and subsystems in the traditional architectures. Each system also has a level of redundancy equivalent to a triple-string configuration, meaning the multicellular architectures have $6 x 3=18$ proteins each. However, each multicellular architecture has its proteins distributed across a differing number of cells. Figures $6.17 \mathrm{~b}$ and $\mathrm{c}$ present similar comparisons but for systems of 12 and 24 tasks respectively.

As seen in Figure 6.16, the most reliable architecture at any point is a multicellular architecture. In addition, when comparing to the plots of Figures $6.17 \mathrm{~b}$ and $\mathrm{c}$, the reliability advantage of multicellular architectures is seen to increase with the total number of proteins 


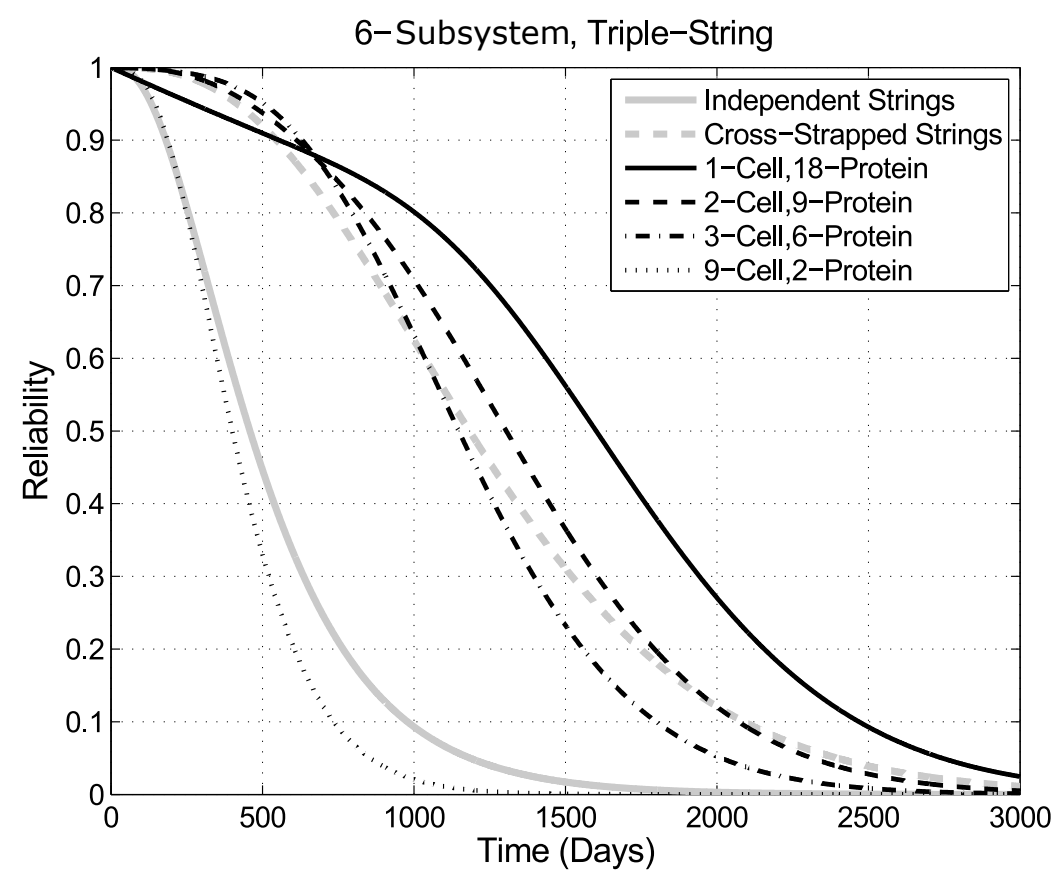

Figure 6.16: Comparison of the sensitivity of various system architectures to total number of tasks. Each system has 6 tasks and a level of redundancy equal to triple-string. In traditional architectures, tasks are performed by subsystems, while in a multicellular architecture they are performed by proteins. At any point in time a multicellular architecture is the most reliable, however, which configuration is optimal changes during the system's lifetime.
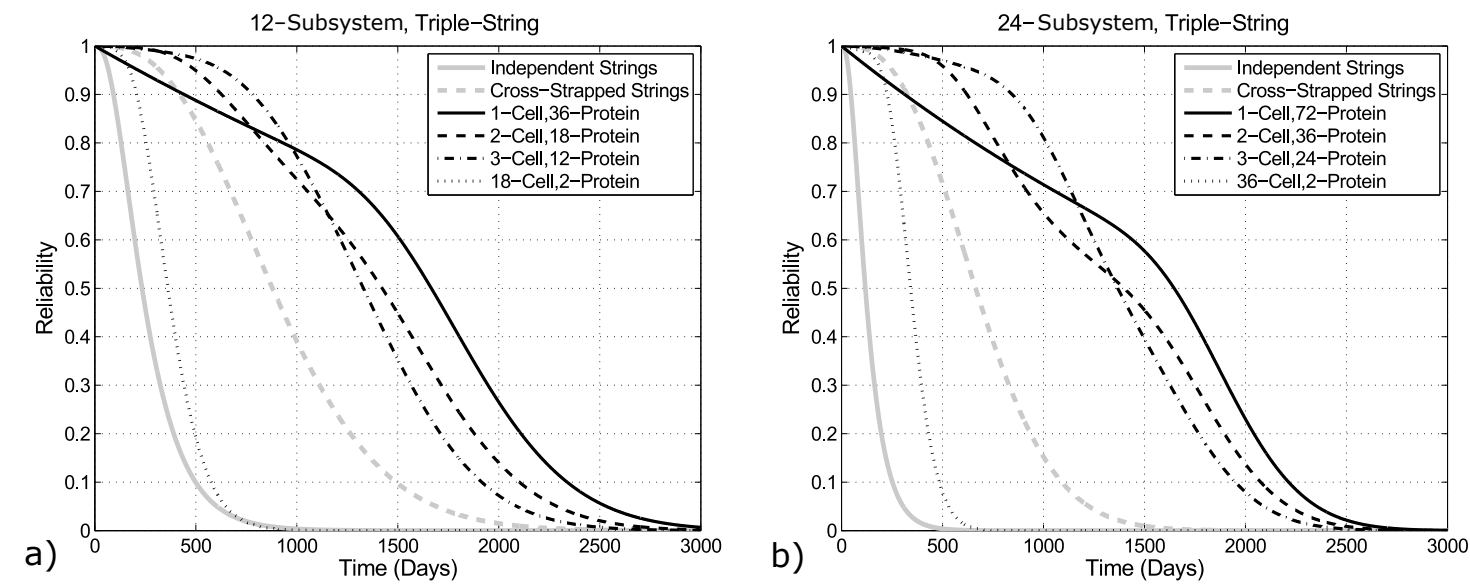

Figure 6.17: Similar to Figure 6.16, but for systems featuring 12 (a) and 24 (b) tasks respectively. The reliability benefit of multicellular architectures is seen to increase with the total number of system tasks. 
in the system. However, which multicellular configuration is the most reliable varies with time. During early life, configurations featuring multiple cells have a reliability advantage, while a single-celled configuration becomes the most reliable later in life.

As seen before in Figure 6.10, the reliability functions of Figures 6.16 and 6.17 display periods of varying failure rates, despite being modelled with constant-failure-rate components. These periods can now be explained as being caused by the peaks and valleys of the system state PDFs. A vertical boundary line can be imaged on the system state PDF which represents the minimum number of proteins required for the system to remain functional. As mission time progresses, the peaks and valleys of the distribution gradually change shape whilst globally moving towards the left. The failure rate is high whilst peaks are moving through the boundary, whilst valleys produce periods of low failure rates.

Based on this description, a system composed of two cells (and therefore featuring two peaks in its PDF) would be expected to have two periods of high failure rates, with periods of low failure rates before, after and in-between. This is exactly what is seen in in the reliability plot for the two-cell, multicellular configuration of Figure 6.17c. The failure rate is proportional to the steepness of the plot, which is seen to drop sharply at approximately 500 and 1500 days. The same pattern is difficult to discern in the reliability plots for the other two-cell configurations because, as explained in Section 6.2.3, these systems feature cells with fewer proteins per cell, which make the peaks in their PDFs less distinguishable.

The reliability plots of three-cell configurations in Figures 6.16 and 6.17 do not show the expected three periods of high failure rates, as in each case the first PDF peak is already left of the boundary line at time zero. This is because, in all three cases, a single cell does not have enough proteins to run all the tasks required for a functional system.

Figures 6.18-6.19 are similar to Figures 6.16-6.17, but in this case sensitivity to system loading is examined. System loading is inversely proportional to spare capacity (or redundancy), and refers to the number of proteins in the system which are executing tasks as a fraction of the total number of proteins in the system. It is evident that cross-strapped and multicellular systems benefit similarly from a reduction in loading. Amongst the multicellular configurations, the benefit of reduced loading is seen to increase with number of cells. This is measured by noting the points at which the reliability functions cross. In Figure 6.19a, featuring systems with a $33 \%$ loading, the triple-cell configuration becomes less reliable than the single-celled configuration once the system reliability has dropped to 0.8. In comparison, in Figure 6.19 b, featuring systems with a $25 \%$ loading, the changeover only occurs at a system reliability of 0.66 . 


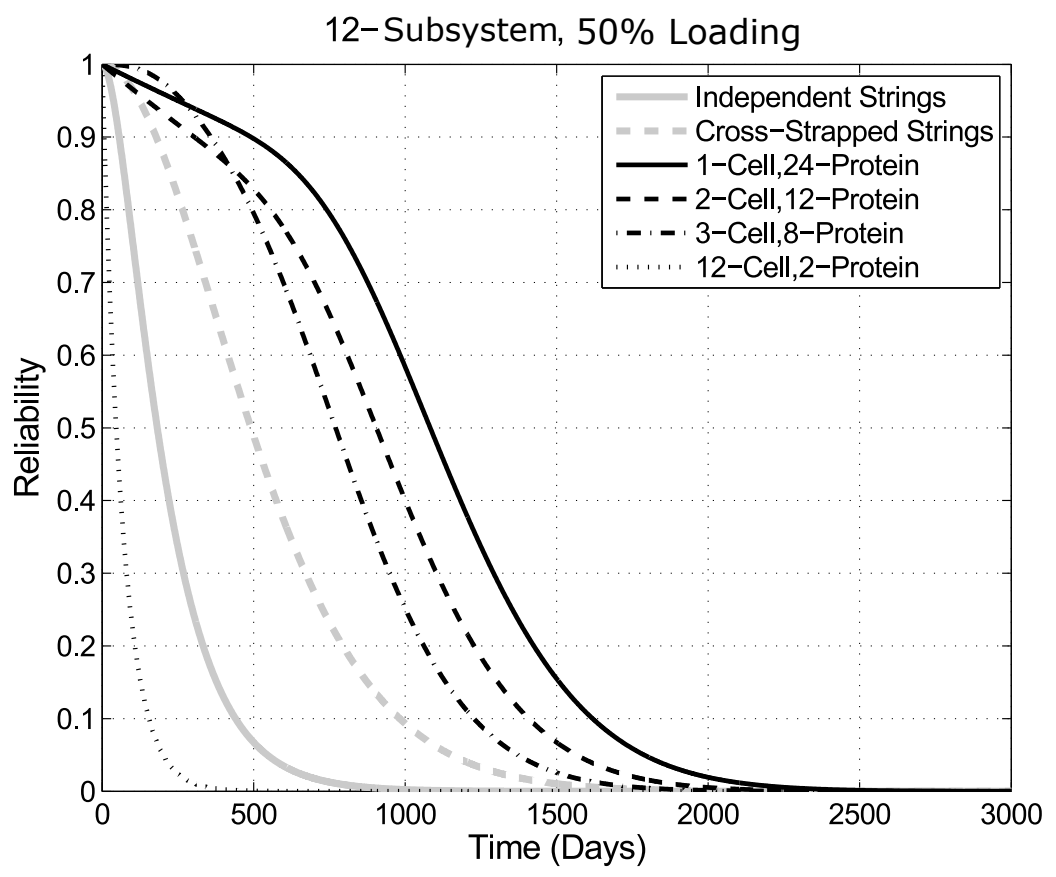

Figure 6.18: Comparison of the sensitivity of various system architectures to loading. Loading is defined as the number of active subsystems or proteins compared to the total number of subsystems/proteins. Each system has 12 tasks to run. At high loading, a single-celled architecture performs best.
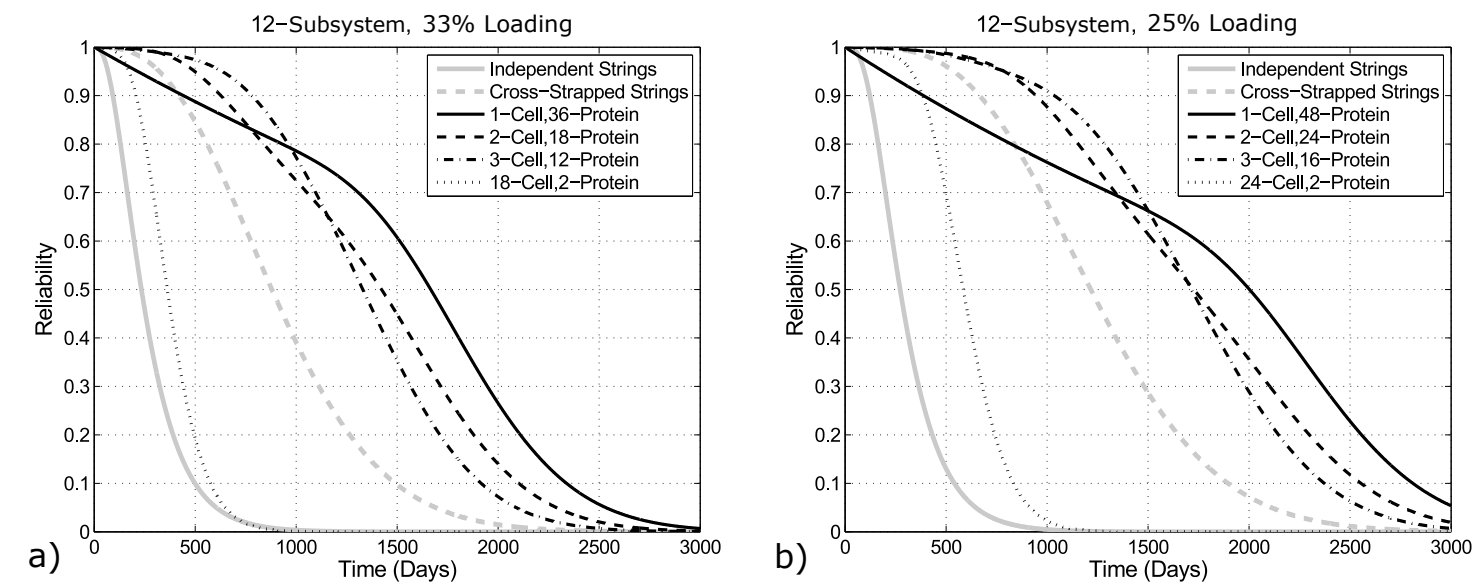

Figure 6.19: Similar to Figure 6.18, but for systems that are $33 \%$ (a) and $25 \%$ (b) loaded. The lower loading is achieved by adding additional strings or proteins. Cross-strapped and multicellular architectures respond similarly to changes in loading. Amongst multicellular configurations, lower loading favours configurations featuring multiple, smaller cells. 
Common component reliability is the next parameter examined. As a reminder, common component failures are those which cause multiple subsystems to fail at once, but are not influenced by the number of subsystems in the system. In the case of multicellular architectures, a common component failure will lead to the loss of a complete cell. Figure 6.20 shows that, in comparison to multi-string and cross-strapped architectures, cellular architectures are more sensitive to common component reliability. Amongst the multicellular configurations, those with relatively few cells benefit more from increased common-component reliability than configurations featuring many cells. This is because configurations featuring many cells are more capable of surviving the loss of individual cells.

Another visible trend is the diminishing returns on increasing common component reliability. For example, the systems in Figure 6.20 have a constant protein failure rate with a MTTF of five years. System reliability is seen to increase appreciably as the common component reliability is increased up to 25 years MTTF (or five times the protein reliability). Thereafter, further increases in the common component reliability yield diminishing returns.

As the common component reliability is lowered to that of the proteins (MTTF of five years, in this case), multicellular architectures lose their reliability advantage over cross-strapped architectures. This is highlighted in Figures $6.21 \mathrm{a}$ and b, which are reliability plots with common component reliabilities from the extreme ends of Figure 6.20's scale. In Figure 6.21a, featuring systems with common component reliabilities equal to their protein reliabilities, it is evident that multicellular and cross-strapped architectures behave similarly. However, as seen in Figure 6.21b, featuring systems with high common component reliabilities, the multicellular architectures display a clear reliability advantage.

Finally, sensitivity to supporting component reliability is investigated. As a reminder, supporting component failures have a similar effect to common component failures. However, the probability of a supporting component failure is proportional to the number of subsystems in the system. In a multicellular architecture it is proportional to the number of proteins per cell and number of cells in the system. Figure 6.22 shows that both cross-strapped and multicellular architectures show a similarly large initial benefit from increased support component reliability. For cross-strapped architectures, the benefits flatten out after increasing the supporting component reliability up to a MTTF of 110 years (20 times the subsystem, or protein, reliability). However, multicellular architectures continue to benefit from supporting component reliability increases far beyond this point. Multi-string architectures, in comparison, are relatively insensitive to supporting component reliability. 


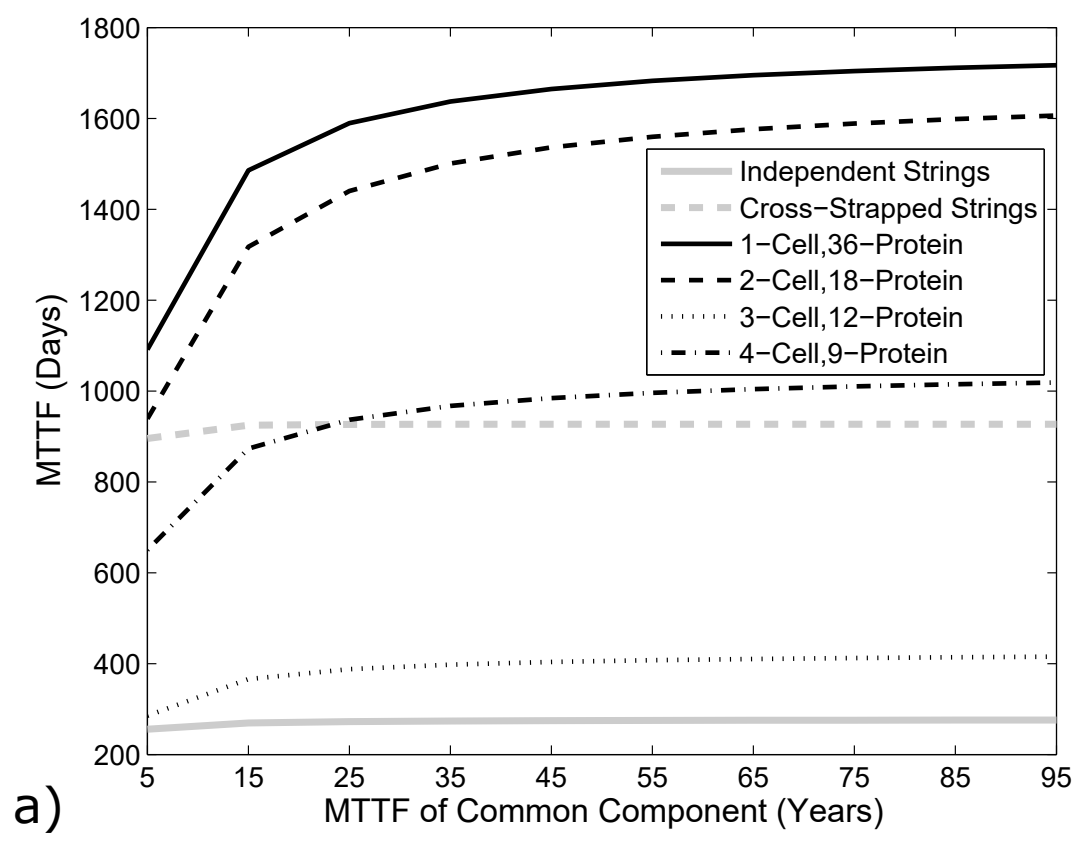

Figure 6.20: Comparison of the sensitivity of various architectures to common component reliability. Here, system reliability is measured with a single metric, MTTF, instead of a whole reliability plot. Multicellular architectures, and especially those featuring few cells, are seen to benefit more from increases in common component reliability than traditional architectures.
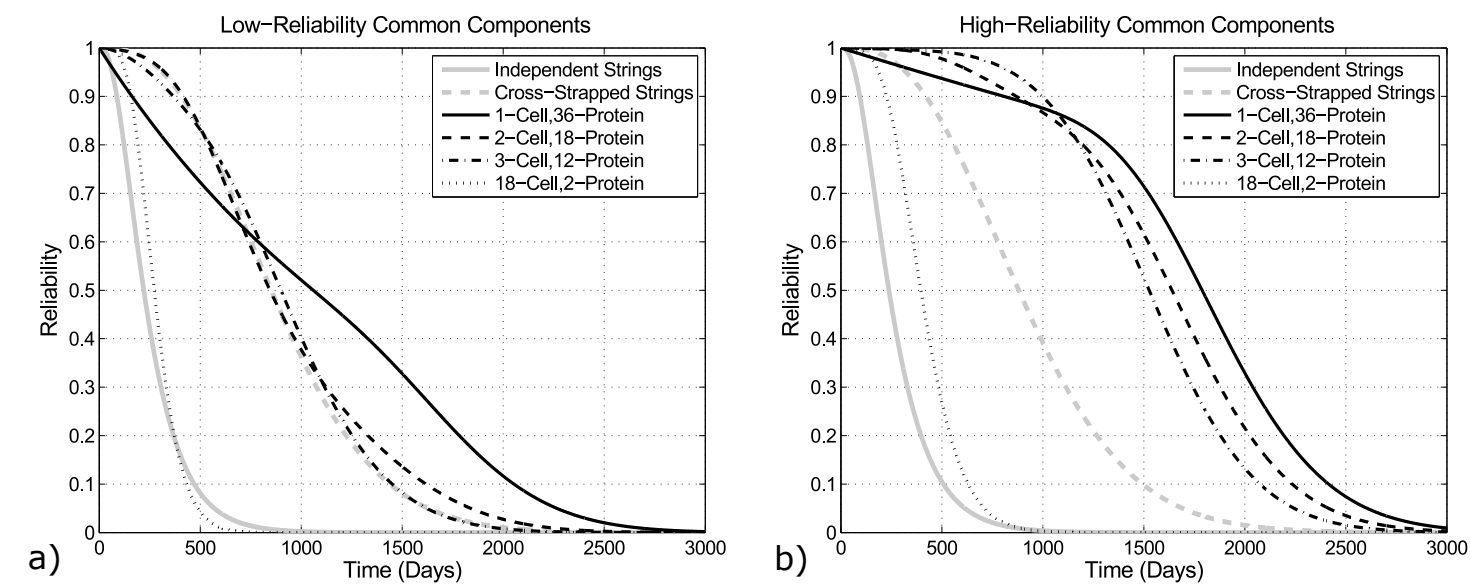

Figure 6.21: Reliability plots of systems with common component reliabilities from the low (a) and high (b) ends of Figure 6.20's scale. At low common component reliabilities (a), multicellular architectures perform comparably to cross-strapped designs. However, at high common component reliabilities (b), multicellular architectures have a clear reliability advantage. 


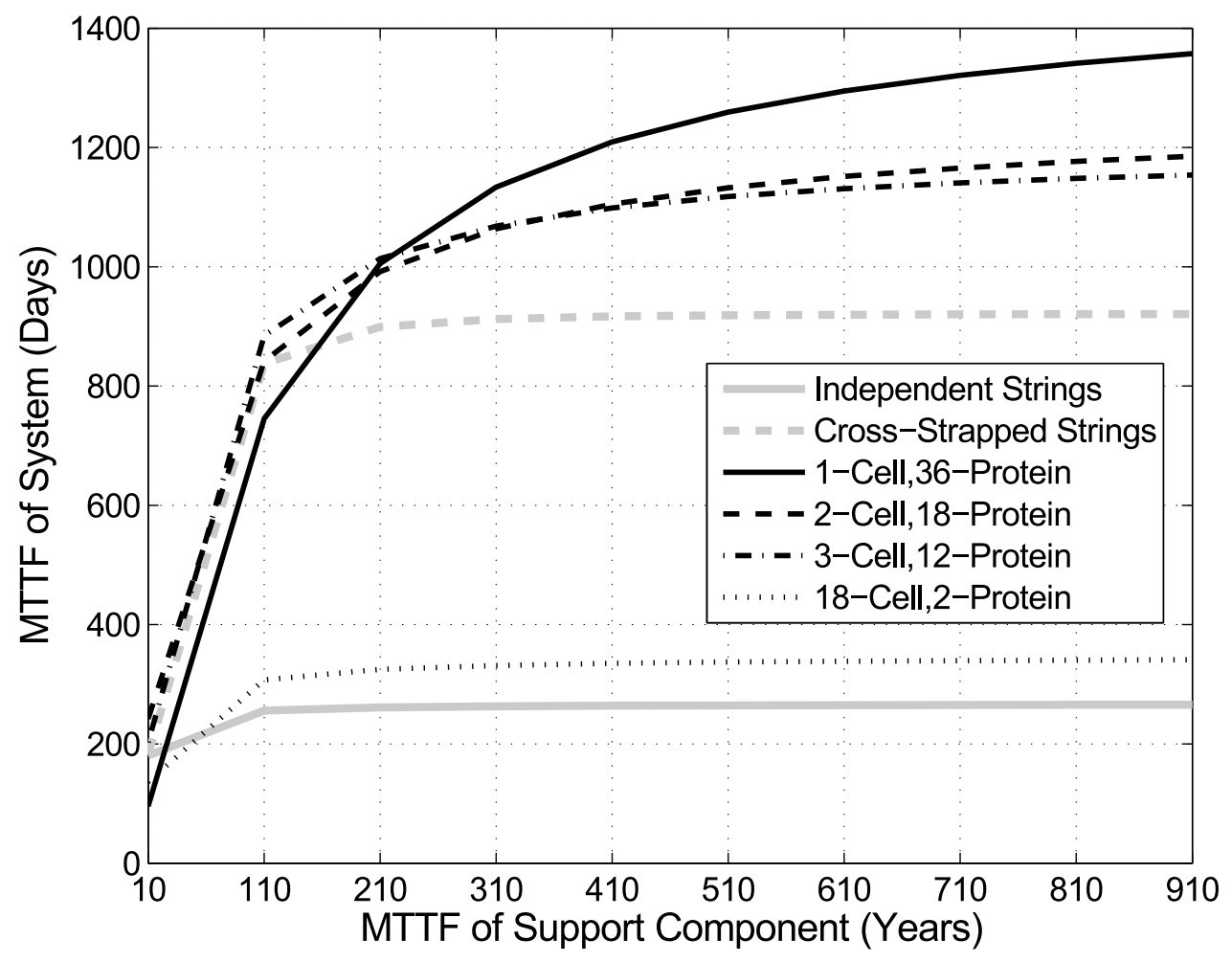

Figure 6.22: Comparison of the sensitivity of various system architectures to support component reliability. Multicellular architectures and cross-strapped architectures benefit similarly from initial increases in support component reliability. Thereafter, compared to cross-strapped architectures, multicellular systems experience more gradual diminishing returns. 


\subsection{Verification through Simulation}

To verify the analytical reliability equations derived in Section 6.2.2, a graphical, multicellular system simulator was developed in MATLAB. The simulator allows Monte Carlo lifetime simulations to be performed on a simulated multicellular system. During each run failures are induced according to a specified distribution, and the simulated system reacts by reconfiguring. Once sufficient failures have occurred that the system cannot reconfigure successfully, the run ends and the simulated mission elapsed time is recorded. Several parameters related to the configuration of the system and the operating environment can be adjusted. These include:

- Number of cells in the system

- Number of proteins per cell

- Number of system tasks to be performed by the proteins (multiple system modes requiring different numbers of tasks can be defined)

- Component failure models (Constant, Exponential, or Weibull)

- Component power requirements and available power

Figure 6.23 shows a screenshot of the main simulation window, which includes a graphical representation of the current system state and various controls for configuring the system and environment. Additionally, controls are provided for manually triggering failures, as well as for stepping through the repair process. Simulations can be set up to run a system till failure once, or a set number of times, with cell and protein failures occurring according to adjustable probability distributions. The simulator is also useful for experimenting with different task allocations strategies. Currently, only the task allocation strategy described in Section 5.2.2 has been implemented, but it would be simple to replace this strategy with others optimised for other variables, such as downtime ore power consumption.

Simulations are relatively slow, with 5000 iterations of a 5-Cell, 7-Protein system taking several minutes on an Intel i5 desktop computer. Simulations involving hundreds of proteins are impractical using this simulator. Therefore, the comparisons made in this subsection are restricted to relatively small systems.

To verify the correctness of the analytical reliability solution derived in Section 6.2.2, reliability functions for various multicellular systems were analytically generated and compared to reliability functions generated through Monte-Carlo simulations in the MATLAB simulator. The failure models used in the simulations are based on Weibull distributions for protein, 


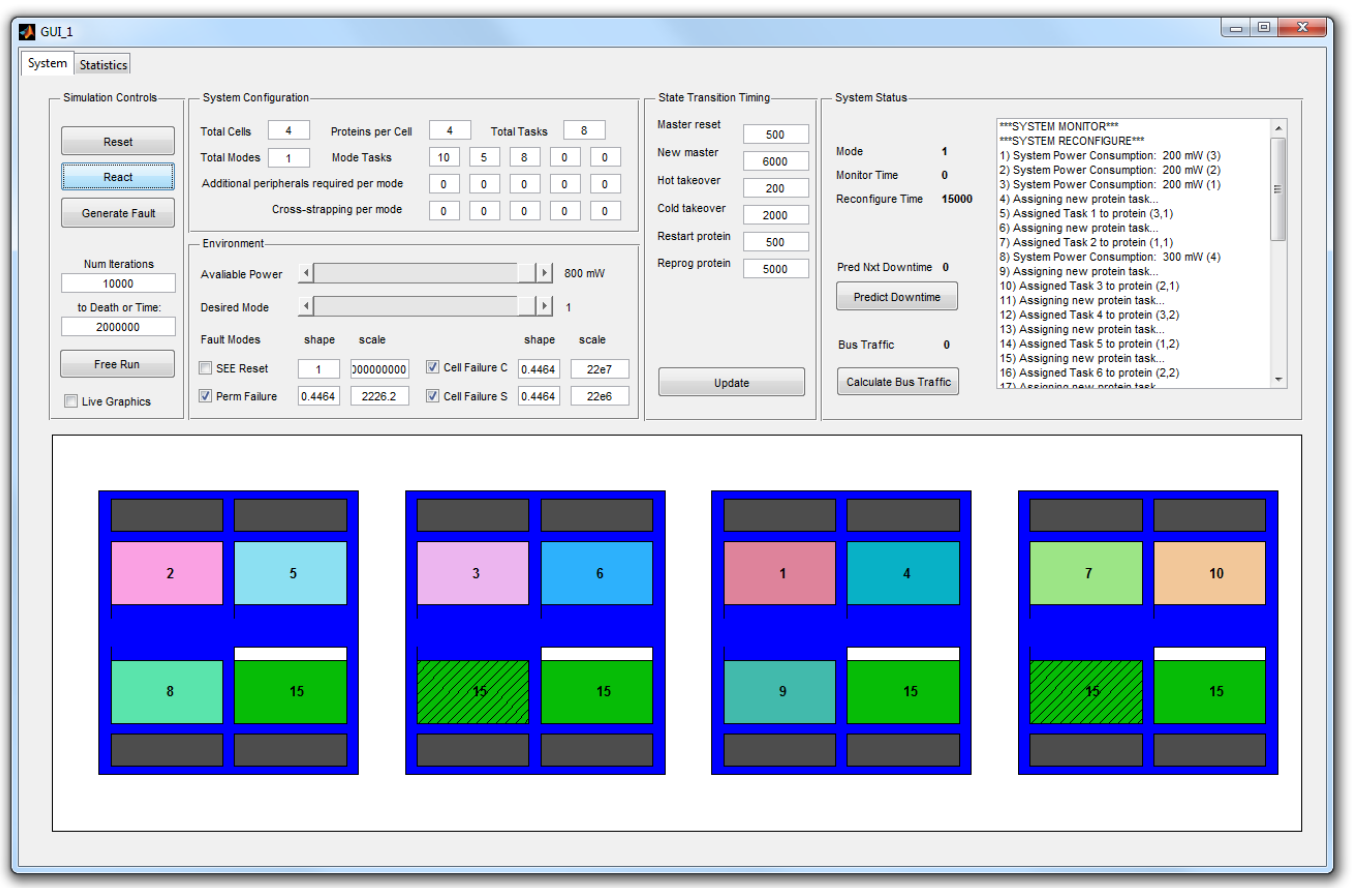

Figure 6.23: The main window of the MATLAB multicellular system simulator. At the top of the window are controls for setting various parameters of the simulated system, as well as controls for stepping through failures and system reconfiguration. The bottom of the window shows a graphical representation of the current system state. In this case, a system composed of four cells of four proteins each is being simulated. 
Table 6.1: Simulated System Failure Models

\begin{tabular}{|l|l|l|l|}
\hline Component & Failure Model & Shape Parameter & Scale Parameter \\
\hline Protein & Weibull & 0.4464 & 2226.2 \\
\hline Common & Weibull & 0.4464 & 220 million \\
\hline Support & Weibull & 0.4464 & 22 million \\
\hline
\end{tabular}

Table 6.2: Comparison between Analytical and Simulated Reliability Functions

\begin{tabular}{|l|l|l|}
\hline $\begin{array}{l}\text { System Configuration } \\
\text { Cells; Proteins/Cell; Tasks }\end{array}$ & Number of Simulations & Reliability Error \\
\hline $4-4-8$ & 1000 & 0.0055 \\
\hline & 10000 & 0.0017 \\
\hline $5-7-10$ & 1000 & 0.0111 \\
\hline & 10000 & 0.0024 \\
\hline $6-5-11$ & 1000 & 0.0127 \\
\hline & 10000 & 0.0019 \\
\hline
\end{tabular}

common and support failures. Their parameters are given in Table 6.1. The shape parameter was taken from an empirical study of nano-satellite failures [26]. The scale parameters are roughly proportional to estimated component complexities and were chosen to give lifetimes similar to those seen in the same study. SEE failures were not simulated as their effect is considered negligible on total system lifetime.

The results of comparisons performed with three different multicellular systems are given in Table 6.2. Each system is described using three numbers (e.g. 5-7-10), which represent the number of cells, proteins per cell, and total system tasks respectively. The error is given as the standard deviation of the error between the analytical and simulated reliability functions, calculated at a resolution of one day. Using 1000 simulations, the errors are on the order of 0.01 , while using 10000 simulations reduces this to 0.001 . The low standard deviation and trend of decreasing error with number of simulation iterations, indicates a good correlation between the analytical and simulated reliability functions.

\subsection{Effect of Peripherals}

While purely computational systems are interesting in their own right, the Satellite Stem Cell hardware has been specifically designed to replace more than just the processing elements of a spacecraft. Every protein has a set of generic I/O circuitry, allowing it to be interfaced 
directly to a variety of peripherals. In this way, complex sensor-actuator systems can be implemented with no additional interfacing hardware.

However, adding peripherals to a system imposes new challenges to the system designer. In a purely computational system, every protein can theoretically run any system task. With the addition of peripherals to the system, this may no longer be true, as the distribution of tasks becomes restricted by which protein has access to which peripheral. In an ideal scenario, a designer may choose to interface every peripheral to every protein. In this case, the system behaves like a purely computational one, and, excluding peripheral failures, can be modelled using the reliability equations of Section 6.2.2.

Unfortunately, cross-strapping every peripheral to every protein is infeasible in all but the smallest systems. The harnessing would be extremely complex and error prone, heavy, and would take up significant amounts of space. Alternatively, some form of multiplexing (MUX) hardware could be designed, but such a solution would present its own challenges regarding who controls the MUX switches and in ensuring the reliability of the potential single-point-of-failure MUX itself.

Thus, as described in Section 4.2, the Satellite Stem Cell architecture does not make use of I/O multiplexers and does not require every peripheral to be cross-strapped to every protein. Instead, the chosen peripheral interfacing solution trades some reliability for implementation simplicity. Every protein can only be interfaced to a single peripheral. However, the GPIO circuitry does allow the same peripheral to be interfaced to several proteins at once. This is possible because all the proteins which have physical interfaces to a particular peripheral, but which are not currently accessing that peripheral, place their GPIO circuitry into a high impedance mode. This prevents interference to the protein which is currently accessing the peripheral. In addition, the GPIO circuitry has been carefully designed to ensure that the majority of its failure modes result in the high impedance state. Thus, a designer can crossstrap peripherals across as many proteins as they see fit. The simplest solution is simply to divide the total number of proteins in the system by the total number of peripherals, and cross strap each peripheral that amount of times. Alternatively, the designer may choose to favour more critical peripherals with more cross-strapping, while reducing the cross-strapping of less critical peripherals.

While this solution favours simplicity, it reduces reliability by restricting which proteins can perform which tasks. To determine the impact of this peripheral interfacing scheme on system reliability, the MATLAB multicellular system simulator was expanded to allow the simulation of peripheral interfaces. In addition to the standard system configuration 

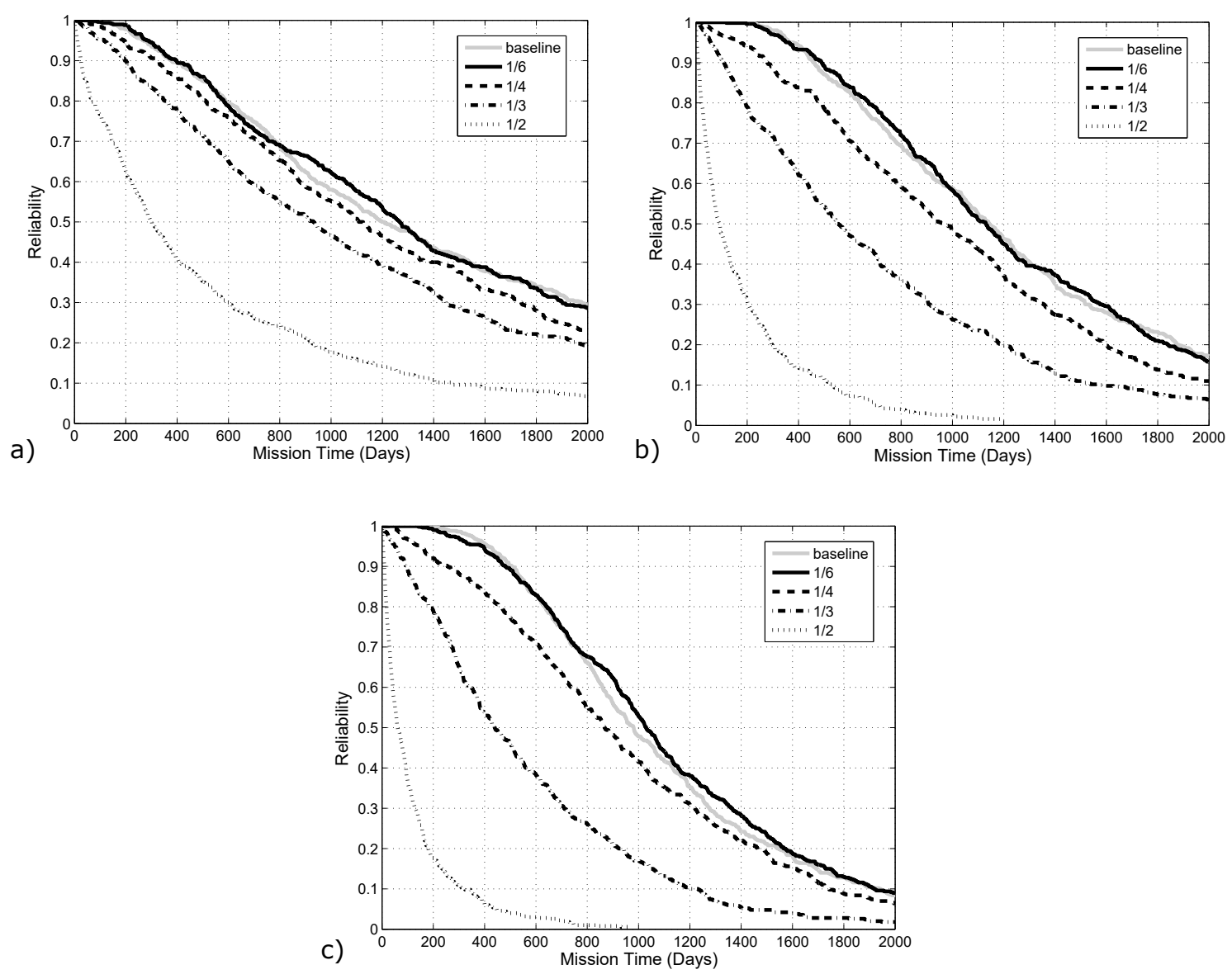

Figure 6.24: The effect of increasing peripheral ratios on three different multicellular systems, composed of 12 (a), 24 (b), and 36 (c) proteins respectively. From all three plots it is clear that increasing peripheral ratios has a detrimental effect on system reliability. However, systems with peripheral ratios below $1 / 6$ can be reasonably approximated by an ideal system.

parameters, the simulator now also allows the number of peripherals and their corresponding level of cross-strapping to be specified.

Three example systems consisting of 12, 24, and 36 proteins, respectively, were simulated . In each case, the system was first simulated without any peripherals to determine a baseline reliability, followed by simulations including an increasing number of peripherals. Other variables, including system configuration and failure models, were kept constant. Each Monte Carlo simulation consisted of 500 iterations. The resultant reliability plots are shown in Figure 6.24a-c.

From all three plots it is clear that system reliability suffers with the addition of peripherals. As more peripherals are added, the reliability function deviates further and further from 
the ideal, purely computation system. In addition, it can be seen that larger systems suffer a greater reduction in reliability from a proportionality equal increase in peripherals than smaller systems. This deviation in reliability from the ideal, purely computational configuration means the reliability predicting equations of Section 6.2.2 can not be accurately used on systems with a high peripheral to protein ratio. Even ratios as low as 1/4 (one peripheral for every four proteins) are seen to cause deviations in reliability of up to $10 \%$. However, for ratios below 1/6, which are shown to be practical in the case studies of Section 7.3, the system may still be approximated by an ideal one. This was shown to hold true regardless of the system loading.

\subsection{Summary}

This chapter focussed on deriving and validating analytical equations for predicting the reliability of multicellular systems. The presented solution is based on an extension of k-out-of-n system theory. Instead of evaluating the system as a whole with binary component functionalities, each cell is evaluated as an independent, multi-state, k-out-of-n system. The full system reliability is then calculated through the convolution of the individual cell results. Determining the optimal configuration of cells per system and proteins per cell proved to be complex, as different configurations were shown to be optimal at different points during a system's lifetime. However, a variety of trends in system behaviour were discovered, which may be useful to multicellular system designers.

Firstly, the reliability benefits of a multicellular architecture over more traditional architectures, such as multi-string and cross-strapped designs, increases with the total number of proteins in the system. In small systems which can implemented using only a few (4) proteins, a multicellular architecture gives little benefit, in terms of reliability, over a cross-strapped one.

Secondly, system loading has a strong effect on the optimal multicellular configuration. Heavily loaded systems ( $>50 \%$ load) should minimise the total number of cells in the system by opting for large cells containing many proteins. On the other hand, systems which are designed to be lightly loaded will benefit from a configuration which features many, smaller cells.

Investigating system sensitivity to support and common component failures showed that multicellular architectures benefit more from increases in the reliabilities of these shared components than traditional multi-string and cross-strapped architectures. If the common 
components have low enough reliability (approximately equal to the reliability of a protein), multicellular architectures perform no better than cross-strapped designs. Thus, it is counterproductive to implement a multicellular system using unreliable communication buses or fragile common circuitry.

The derived multicellular reliability equations were verified using a multicellular system simulator developed using MATLAB. The simulator has a graphical user interface and allows Monte Carlo simulations (composed of thousands of runs) to be performed with a number of configurable parameters. Comparisons between the reliability functions calculated using the analytical equations and those produced by the simulator showed a good correlation.

Using the same simulator, the effect of peripherals on the reliability of multicellular systems was investigated. The ability to interface directly to a variety of peripherals is one of the most important features of the Satellite Stem Cell architecture. However, adding peripherals to a system causes a negative deviation from the analytically predicted reliability, as the peripheral interfaces limit reconfigurability. Nevertheless, it was shown that the derived reliability equations are still useful for predicting the reliability of multicellular systems with peripheral ratios below $1 / 6$.

Given a multicellular architecture is chosen, there are many additional aspects to consider when determining the configuration of cells per system and proteins per cell. These include physical constraints such as available volume, bandwidth constraints on internal communication buses, and implementation constraints (e.g. limited I2C addresses). However, it is believed that the results obtained in this chapter will allow systems developers to make some early decisions, such as whether a multicellular architecture is appropriate, and will aid in determining an optimal configuration. 



\section{Chapter 7}

\section{Multicellular System Implementations}

In previous chapters, the hardware and software aspects of the Artificial Stem Cell Architecture were described in detail, together with an analytical and simulation-based investigation of its theoretical reliability. In this chapter, two practical implementations of the ASC Architecture will be described.

The first implementation can more accurately be described as version 0.1 of the ASC Architecture. It was developed as a solution for interfacing to a large number of sensor and actuator payloads on the SMESAT CubeSat, which was being developed by the Surrey Space Centre. While this implementation lacks many features of the full ASC Architecture, including the HARP middleware and generic I/O circuitry, it nevertheless demonstrated many of the benefits of a multicellular approach. This chapter includes a brief overview of the SMESAT CubeSat and the design and development of its cellularised payload interface computer.

The second implementation of the ASC Architecture takes the form of a two-cell, benchtop multicellular system demonstrator. It fully implements all aspects of the proposed artificial cells and agent-based middleware. Together with implementation details, this chapter describes a set of experiments which aimed to test the functionality and performance of the benchtop multicellular system.

Finally, based on practical measurements of the benchtop system and the results of the reliability analysis of Chapter 6 , two case studies of cellularising portions of real spacecraft are given. 


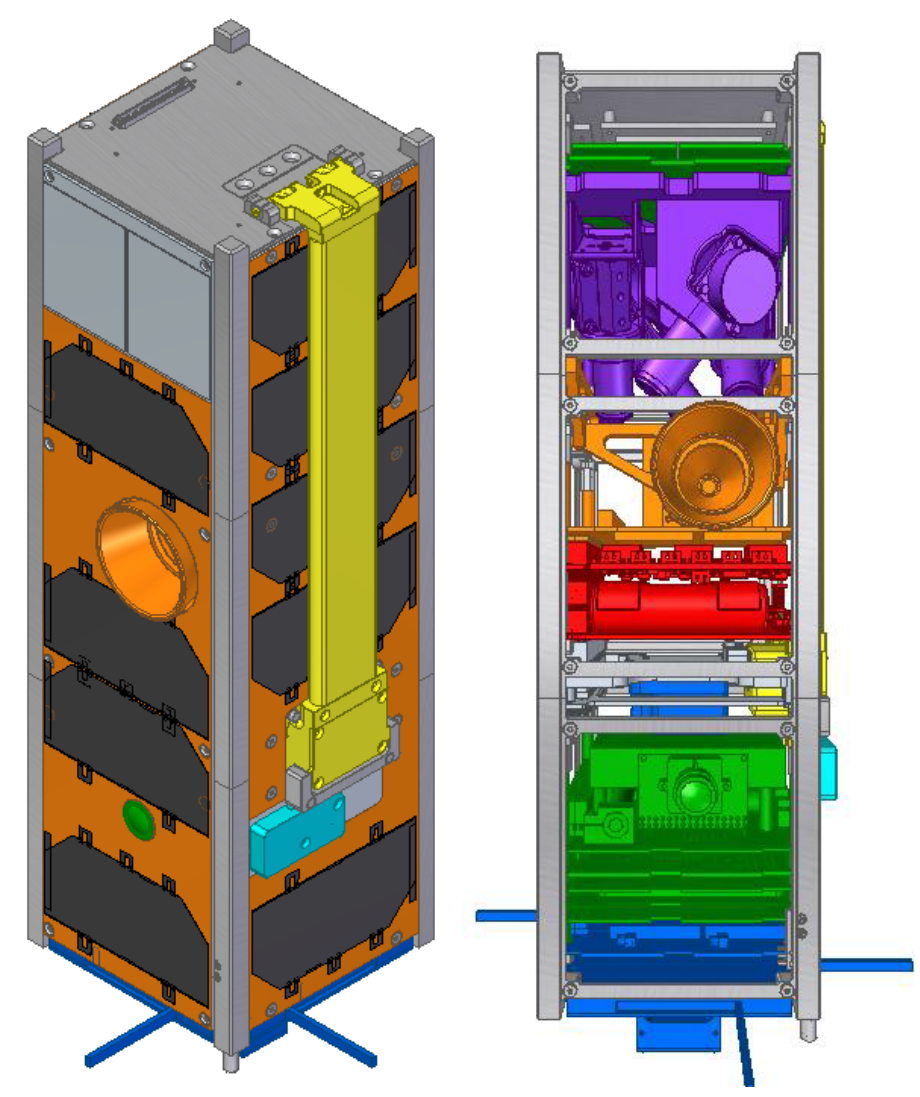

Figure 7.1: A computer model of the SMESAT CubeSat being developed by the Surrey Space Centre. SMESAT's primary goal is to space-rate payloads from various small and medium enterprises across europe and Canada.

\subsection{SMESAT}

SMESAT, shown in Figure 7.1, is a CubeSat developed by the Surrey Space Centre as part of a European Commission FP7 project. The satellite measures approximately $10 \mathrm{x} 10 \mathrm{x}$ $30 \mathrm{~cm}$ and carries a number of payloads from Small and Medium Enterprise (SME) project partners. The primary aim of the SMESAT mission is to test the performance of the payloads on orbit so that they may be sold as commercial products in the future. Traditionally, 'spacerating' a component is a complex and expensive procedure which may involve a variety of environmental and radiation test campaigns, as well as trial flights. SMESAT aims to demonstrate that space-rating a component by flying it on a CubeSat may provide a faster and more cost effective route to market.

The SMESAT payloads are diverse and come from a variety of European and Canadian SMEs. They include: 
- STIM300 three-axis, high-accuracy, MEMs inertial measurement unit (IMU) by Sensonor in Norway.

- High-accuracy MEMs accelerometers by Theon Sensors in Greece.

- Minitature, science-grade fluxgate magnetometer by Laboratory of Electromechanical Innovation (LEMI) in Ukraine.

- CubeSat star tracker by Innovative Solutions in Space in the Netherlands.

- Smart Thermal Radiator Tiles by MBP Communications Inc in Canada.

- Nano Control Moment Gyroscope (CMG) Array by Surrey Space Centre in England.

- CubeSat EPS by SystematIC in the Netherlands

\subsubsection{Initial System Design}

Like many amateur satellites, SMESAT is employs the single-string system architecture and is composed of a mixture of COTS and custom-designed subsystems. The spacecraft bus is composed of a COTS communications subsystem and antennas from ISIS (shown in blue in Figure 7.1), a COTS combined OBC and attitude control subsystem from CubeSpace (green), and the experimental SystematIC EPS (red). In addition, due to the large number and diversity of payloads, the system contains a custom payload interface computer (PIC). The PIC is responsible for operating the payloads on orbit and for collecting experiment data for later transmission to the ground.

The initial design of the PIC called for a single $10 \times 10 \mathrm{~cm}$ PC/104 PCB which could interface to and operate the STIM300, LEMI magnetometer, Theon accelerometers and Smart Thermal Radiator Tiles temperature sensors. In addition, since SSC was also providing its own payload, the nano CMG array, it was expected that the PIC would provide the drive and control for this payload, too. These interfaces are summarised in Table 7.1.

It was quickly realised that designing the hardware and firmware of the PIC would be complex and likely take longer than the schedule allowed. In addition, the PIC was seen as a worrying potential single point of failure, as it is the sole interface to a large fraction of the payloads and is itself a new development. Thus, based on the Satellite Stem Cell work presented in this thesis, an alternative design for the PIC was proposed and implemented. 
Table 7.1: SMESAT Payload Interface Computer required interfaces

\begin{tabular}{|l|l|}
\hline Payload & Interface \\
\hline STIM300 & RS422 \\
\hline LEMI Magnetometer & $3.3 \mathrm{~V}$ RS232 \\
\hline Theon Accelerometers & I2C \\
\hline Smart Thermal Radiator Tiles & $4 \times 3.3 \mathrm{~V}$ analogue inputs \\
\hline Nano CMG Array & $3 \times$ high-current outputs \\
4 x BLDC Motor & $3 \times$ digital hall-effect sensor inputs \\
& $4 \times$ high-current outputs \\
4 x Stepper Motor & $1 \times 3.3 \mathrm{~V}$ analogue inputs \\
4 x Absolute Encoder & \\
\hline
\end{tabular}

\subsubsection{Cellularised Design}

The new PIC design is based on the artificial cell concept. It is composed of a single cell of four proteins. Each protein is electrically identical and interfaces to a single CMG and a single additional payload. This design is shown in Figure 7.2.

Each protein is based on a simple ARM Cortex M0 MCU clocked at $48 \mathrm{MHz}$, and an external $1 \mathrm{MBit}$ of EEPROM memory. The memory is used for experiment data and alternative firmware images. The proteins are linked by a single CAN bus, which also serves as the interface to the CubeSat OBC. Every protein can be in one of several modes, depending on whether it is running experiments related to its external payload, or controlling its CMG. The four proteins were designed as daughter boards which plug into a PC/104-sized carrier board. The carrier board distributes power to the proteins and routes protein I/O signals to physical connectors. The PCB assembly is mounted to the bottom of the CMG array mechanics, as shown in Figure 7.3.

To limit the risk and experimental nature of the PIC, the HARP middleware was not implemented. Instead, simple, bespoke firmware was written for each protein based on its attached peripheral, with a large percentage of the code being common to all proteins. Every protein has the same code for controlling its attached $\mathrm{CMG}$, and every protein has the ability to be master of the CMG array. As master, the protein is responsible for coordinating the behaviour of the CMG array as a whole, and for logging resultant data. While proteins are capable of communicating directly with one another and even reprogramming each other, it is envisioned that the proteins will be commanded and, if necessary, reprogrammed, directly from the CubeSat OBC. Thus, the cell lacks the proposed macromolecular machinery of Chapter 5. 


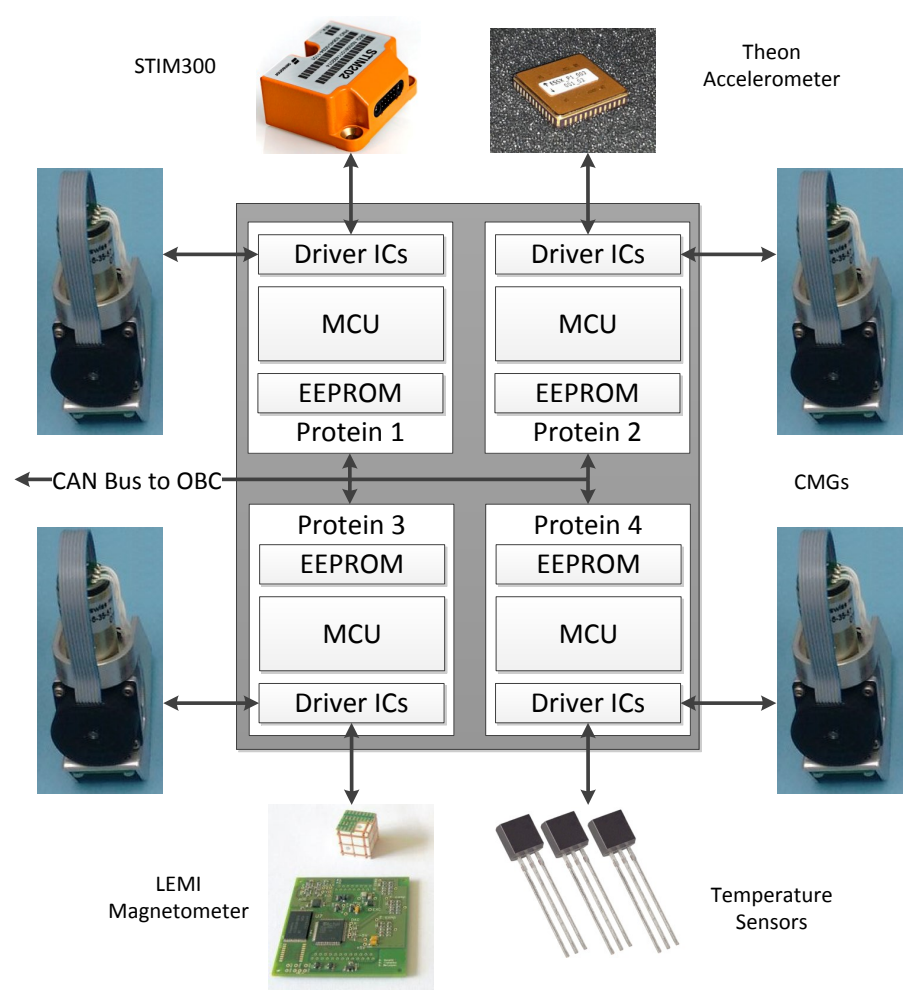

Figure 7.2: Schematic of the cellularised payload interface computer concept.

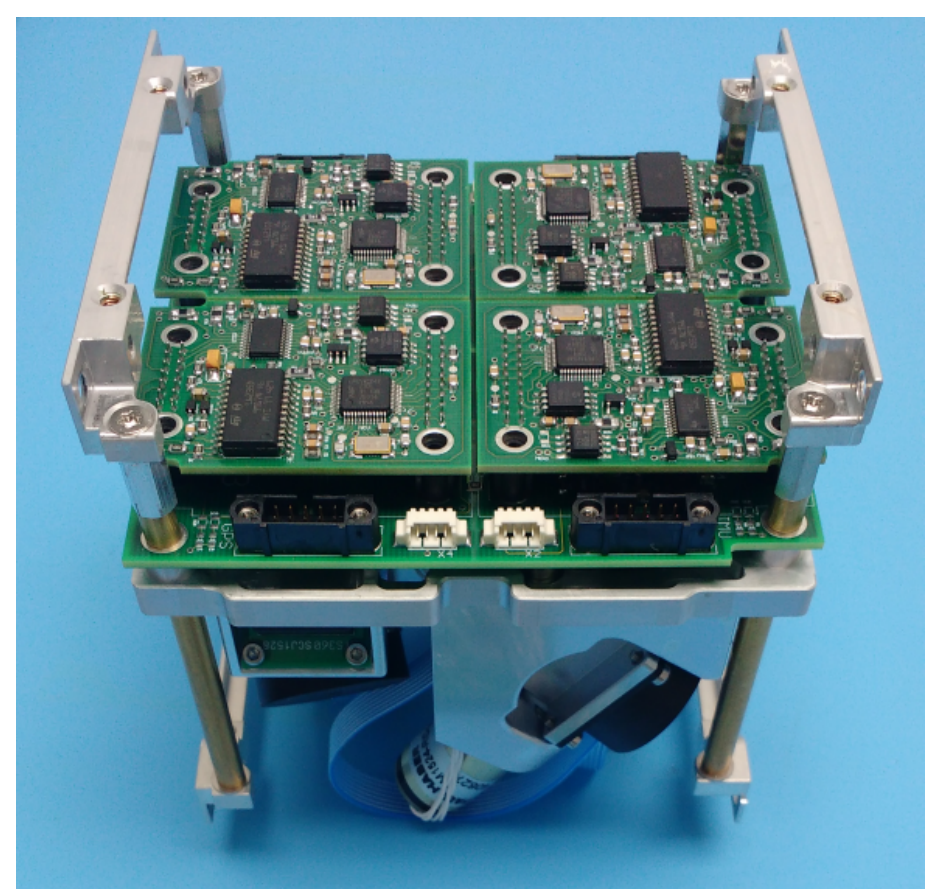

Figure 7.3: Flight version of the cellularised PIC, mounted on top of the nano-CMG array. Note the four identical protein PCBs. 
Furthermore, the proteins of the PIC do not contain generic I/O circuitry. Instead, discrete motor driver and bus transceiver ICs provide the interfaces to the CMG actuators and payloads. Initially, cross-strapping payloads between proteins was considered, but, as discussed in Chapter 4, without generic I/O circuitry this would require complicated multiplexing and harnessing. Since the loss of a single payload due to the failure of a protein or interface should not affect other payloads, the one-payload-per-protein configuration was deemed acceptable.

Despite all these simplifications and limitations compared to a full artificial cell implementation, the cellular PIC design demonstrated the following advantages:

1. Simplified hardware design - duplicated, simple design, based on low-pin-count MCU, instead of complex design based on large MCU.

2. Simplified and paralleled firmware development - no multitasking concerns and code for each payload could be developed and tested in parallel.

3. Graceful degeneracy in experimental output - failure of a protein causes loss of only a single payload and CMG.

4. Flexible power consumption - each active protein consumes only $66 \mathrm{~mW}$, while idle proteins can be turned off to conserve power.

The PIC was developed in parallel to the other work presented in this thesis over the course of a year, while the firmware was written with the help of an additional programmer over a few months. The SMESAT CubeSat is currently largely complete and in the soft-stack stage, as seen in Figure 7.4, and awaiting environmental testing and a launch opportunity.

\subsection{CubeSat-Scale Multicellular System Testbed}

After the development of the SMESAT cellularised Payload Interface Computer, work progressed onto a more comprehensive CubeSat-scale multicellular testbed. It was developed to demonstrate the full functionality of the ASC hardware and HARP middleware, and to determine its real-world performance and implementation overheads. The testbed was purposefully designed at CubeSat scale so comparisons could be made to heritage CubeSat subsystem hardware, which is available in the SSC. The following subsections describe hardware and middleware implementation details, as well as a set of experiments which were performed on the testbed to characterise its performance. 

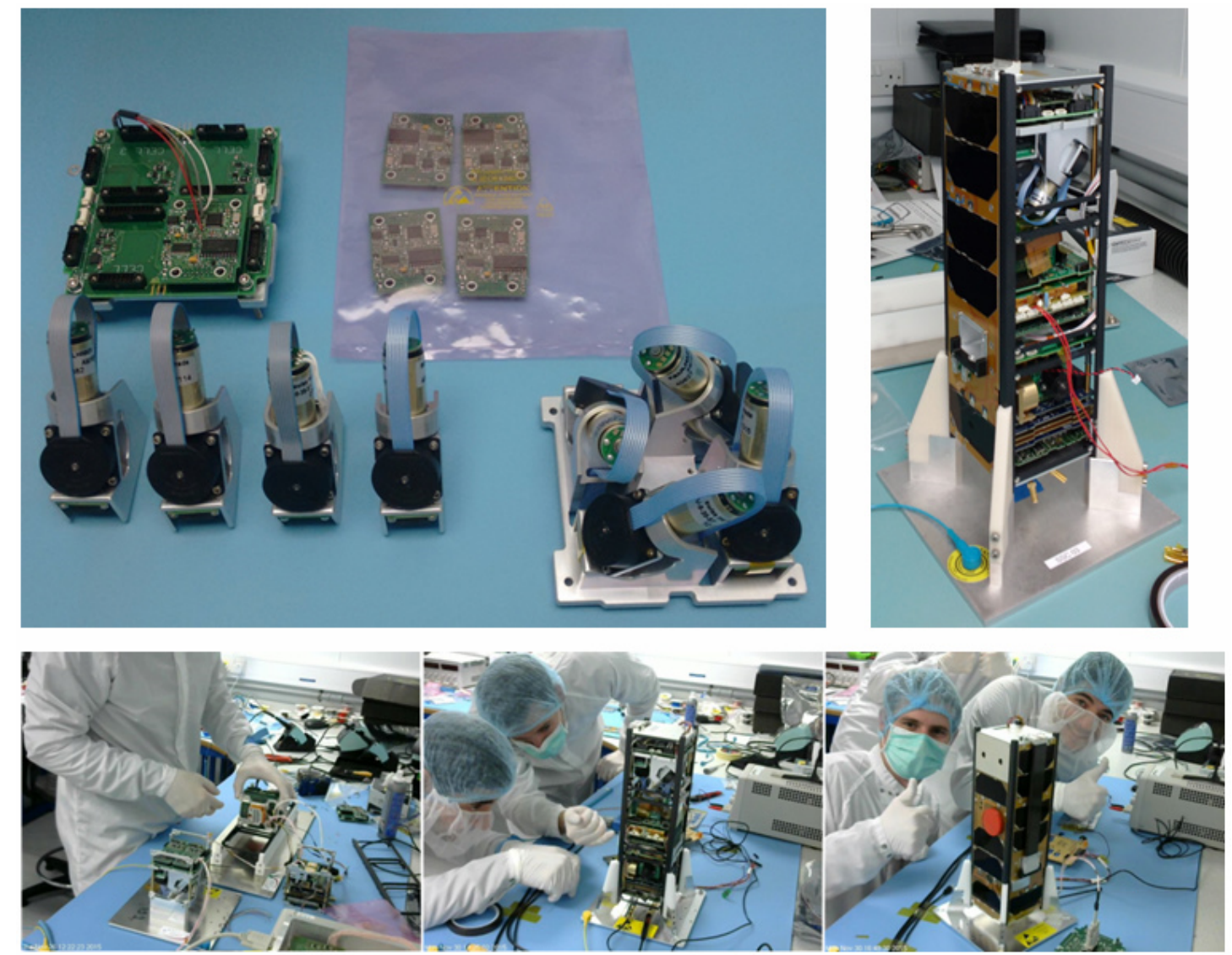

Figure 7.4: Clockwise from top left: All of the components of the PIC and CMG array, soft-stacked SMESAT, assembly montage. 


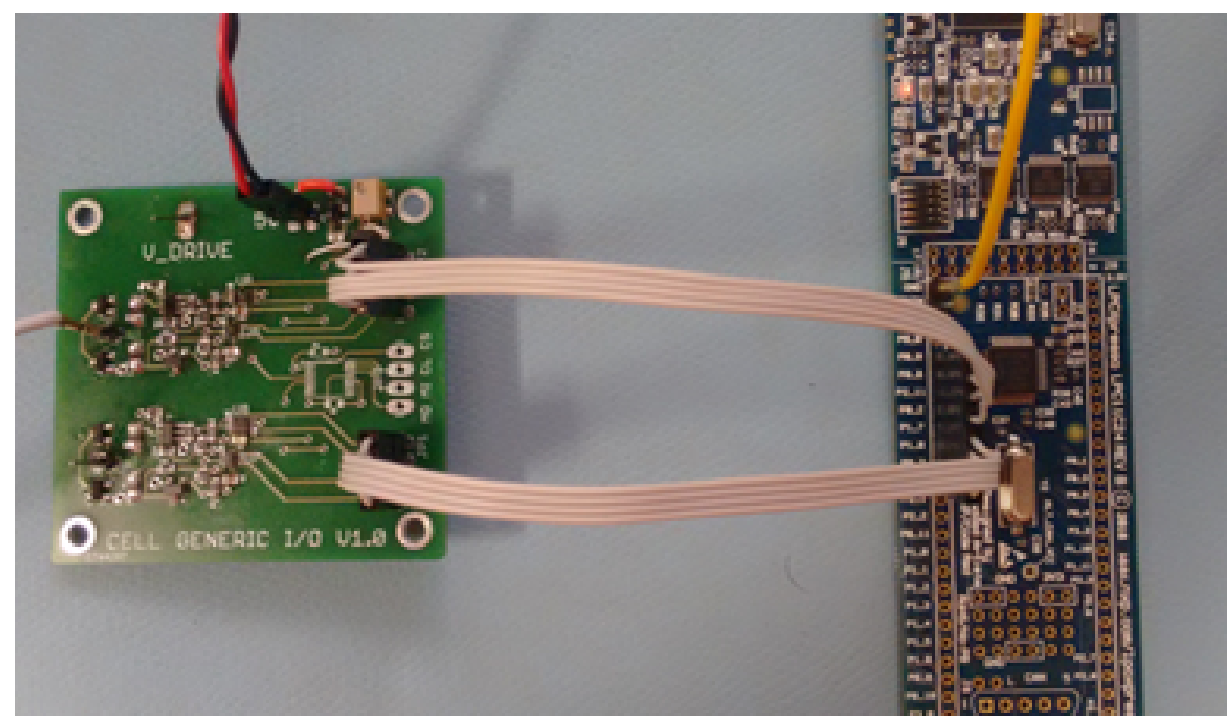

Figure 7.5: Prototype generic I/O circuitry.

\subsubsection{GPIO Circuitry Prototyping}

The generic I/O circuitry, which is the artificial counterpart of a biological cell's membrane, is one of the most novel features of the Satellite Stem Cell hardware. Its design is crucial for ensuring that an artificial cell can adapt to interface to a wide variety of peripherals, and its physical size has a large impact on the overall cell size. Therefore, a prototype of the generic I/O circuitry, consisting of two I/O channels, was implemented first. This prototype can be seen in Figure 7.5, with an MCU board controlling its behaviour.

In line with the desire to have the testbed be comparable with CubeSat avionics, each channel of prototype generic I/O circuitry was designed with the following specifications:

- Supports output voltages between $3.3 \mathrm{~V}$ and $9 \mathrm{~V}$

- Provides drive and sink capability of at least 1A

- Provides current measurements when sourcing or sinking

- Supports digital I/O bit rates of at least $100 \mathrm{~kb} / \mathrm{s}$

- Supports digital and analogue input voltages between $3.3 \mathrm{~V}$ and $9 \mathrm{~V}$

- Supports a high-impedance state

In addition, the following two specifications were aimed for, but only partly achieved:

- Minimal physical changes required for different operating modes 
- No single component failure should prevent entry into the high-impedance state

The I/O bit rate specification is to ensure compatibility with I2C buses, which are commonly used in CubeSats. The supported voltages specification is to ensure compatibility with $3.3 \mathrm{~V}$ and $5 \mathrm{~V}$ interfaces, as well as to support switching peripherals directly from the battery voltage, which on CubeSats is commonly around 7.2V. One Amp of current sourcing and sinking capability is required to drive magnetorquers and the motors of reaction wheels, while the high impedance state is required for analogue input voltage sensing, and when acting as a backup for a cross-strapped peripheral.

A key aim of the Satellite Stem Cell Architecture is reduced manufacturing costs and simplified testing through cell mass production. Thus, ideally, each cell should be electrically identical. This implies that the generic I/O circuitry should support all the modes of operation described above without requiring any physical rework. Such multifunctional I/O circuitry would be possible with the use of digitally controlled analogue switches and multiplexers. However, since the operational mode of each channel of I/O circuitry is fixed as determined by its attached peripheral, there is no need to be able to change mode once operational. In fact, once the system is operational, the analogue switches and muxes become a risk. Thus, the design of the I/O circuitry must represent a compromise. In this implementation of the I/O circuitry, analogue switches and muxes are avoided in favour of small physical changes, such as installing jumpers or choosing a minimal set of resistors.

The generic I/O circuitry implementation closely follows the block diagram of Figure 4.2 in Chapter 4. A section of the schematics (excluding the drive logic, ADC and schmitt trigger ICs) is given in Figure 7.6. The design was tested through simulation in LTSpice before moving on to the physical implementation.

I/O protection is provided by two transient voltage suppression (TVS) diodes (D2 and D3), which guard against over-voltage transients and electro-static discharges from handling. In addition, a schottkey diode prevents backpowering in the event that external voltages on the $\mathrm{I} / \mathrm{O}$ line exceed the local rails. To protect the schmitt trigger and analogue stages, the $0-9 \mathrm{~V}$ full-scale I/O range is scaled to $0-3.3 \mathrm{~V}$ by a set of voltage dividers.

Output drive and sink capability is provided by a pair of p-channel and n-channel MOSFETS in a half-bridge configuration. A set of discrete logic gates provide the drive logic and prevent the possibility of shoot-through. Brushed-DC motor control was demonstrated using both prototype generic I/O channels to form a full $\mathrm{H}$-bridge. All the standard $\mathrm{H}$-bridge drive modes, including forwards, reverse, brake and coast, were successfully demonstrated, as shown in Figure 7.7. 


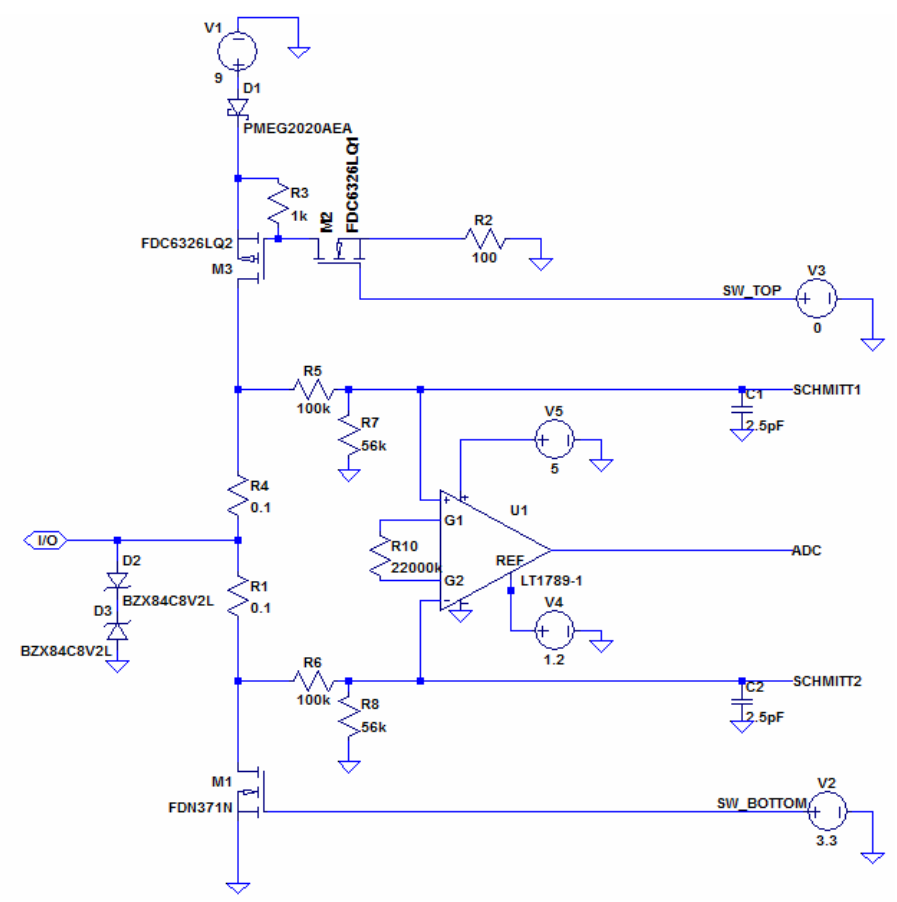

Figure 7.6: A section of the generic I/O circuitry, showing the half-bridge output stage and instrumentation amplifier input stage.

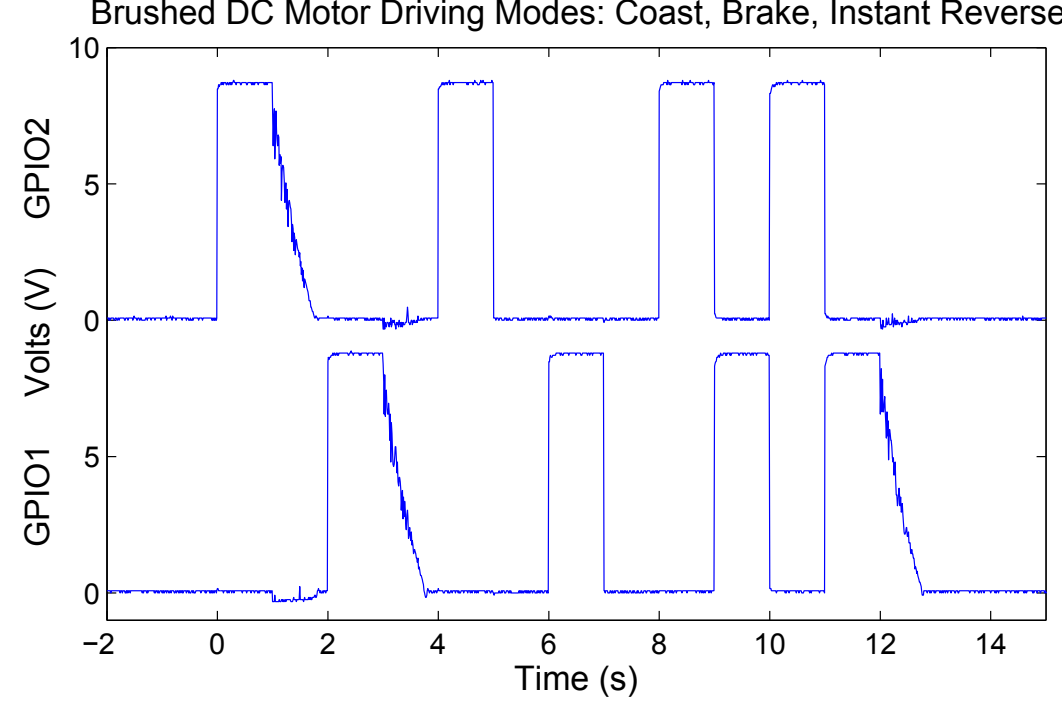

Figure 7.7: Demonstration of a pair of prototype generic I/O channels driving a brushed-DC motor in full h-bridge configuration. 


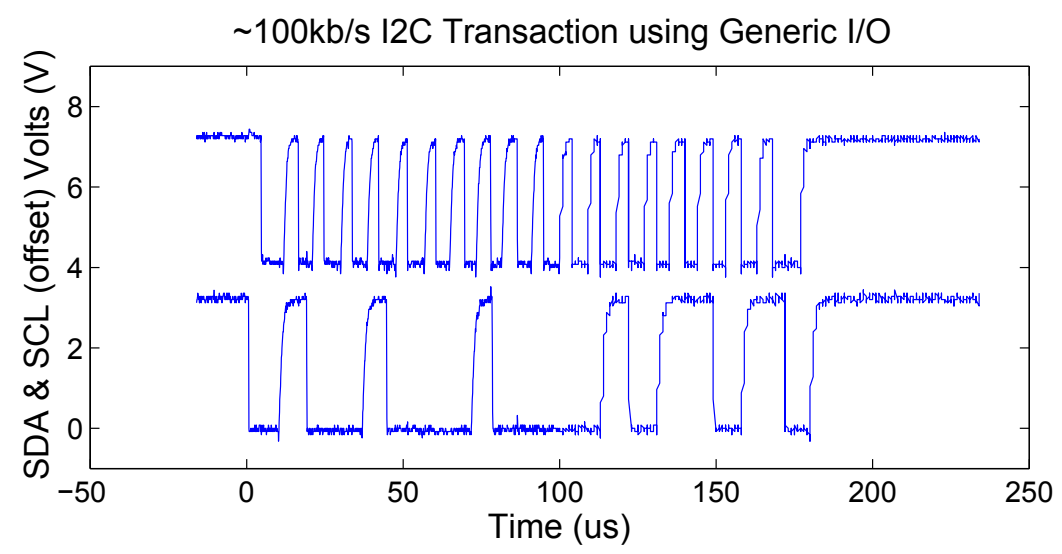

Figure 7.8: Demonstration of a pair of prototype generic I/O channels completing a $100 \mathrm{~kb} / \mathrm{s} \mathrm{I} 2 \mathrm{C}$ transaction.

A challenge was finding small-package MOSFETs capable of switching at high speed (hundreds of $\mathrm{kHz}$ ) and handling large currents. A sufficiently fast n-channel MOSFET was found, but the p-channel MOSFET is too slow for $100 \mathrm{kHz}$ switching. Fortunately, interfacing to an $\mathrm{I} 2 \mathrm{C}$ bus only requires pull-down capability as the bus is normally held high by pull-up resistors. Thus, the prototype generic I/O circuitry was capable of successfully performing $\mathrm{I} 2 \mathrm{C}$ transactions at $100 \mathrm{~kb} / \mathrm{s}$. An example transaction with an $\mathrm{I} 2 \mathrm{C}$ temperature sensor, captured with an oscilloscope, is shown in Figure 7.8.

An instrumentation amplifier, (U1 in Figure 7.6), lies at the heart of the analogue input stage. It has two modes of operation. Firstly, it can be used to measure the current flowing through the output stage. In this configuration, the voltage drop over a pair of sense resistors (R1, $\mathrm{R} 4)$ is measured and a large gain is required. The gain is set using a single resistor (R10), which on the prototype I/O circuitry is replaced by a potentiometer to allow the gain to be adjusted easily. In its second mode of operation, the instrumentation amplifier is used to directly measure input voltages. To configure the channel for this mode, the bottom sense resistor must be removed. Then, by turning on the bottom MOSFET in the output stage, the negative input of the amplifier is referenced to ground. Any external voltage applied to the I/O channel will then be amplified by the the amplifier and presented to the ADC. In this mode, a variety of gains may be required, depending on the analogue voltage source.

The ability to cross-strap peripherals relies on the I/O channel high-impedance state. An I/O channel which fails in such a way that it is permanently high or permanently shorted to ground will block all access to the peripheral, regardless of cross-strapping. Thus, it is critical that the I/O circuitry's failure modes are designed to avoid this eventuality. The design goal is that no single component failure should result in this undesired state. 
To study the I/O circuitry failure modes, a fault tree analysis was undertaken. The resultant fault tree is shown in Figure 7.9. At the top of the tree is the undesired I/O channel state, which represents being stuck in any state other than high-impedance. The second level of the tree breaks this down into two states: stuck high or stuck shorted to ground. The lower levels of the tree contain all the combinations of component failures which could lead to one of these two states. In almost every case, a combination of at least two component failures is required to end up in an undesirable output state. However, a careful look at the tree will reveal that the TVS diodes fail the design goal. A shorted failure of the lower TVS diode will short the I/O channel to ground. The simple nature of the diode as a component makes its failure very unlikely and this slight departure from the design goal is therefore accepted. However, if deemed necessary, another diode in series would remove this weak point.

In addition to demonstrating functionality and testing performance, one of the main aims of the prototype I/O circuitry implementation was to judge how compact, in terms of PCB area, the circuitry could be made. A practical artificial cell requires each of its proteins to have at least $8 \mathrm{I} / \mathrm{O}$ channels (based on Table 4.1 in Chapter 4). Thus, in a CubeSat-scale cell (10 x 10 $\mathrm{cm}$ ), the PCB area available per I/O channel is very limited. Once laid out, each prototype I/O channel fit into an area of approximately 13 x $30 \mathrm{~mm}$, which was deemed sufficiently compact. Thus, all functional and physical requirements were met to proceed to full cell design. 


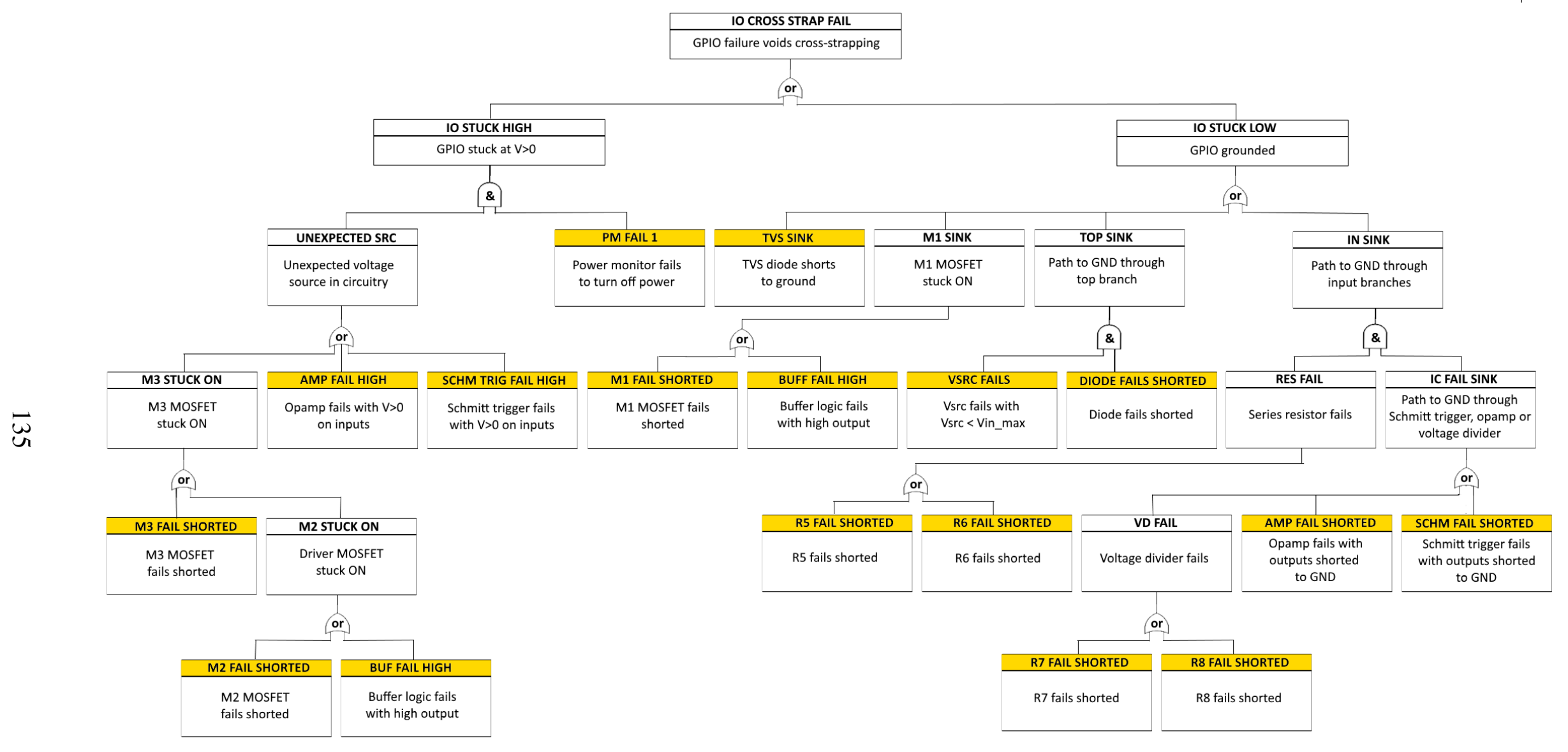

Figure 7.9: Fault tree analysis of the generic I/O circuitry. Only the TVS diode presents a potential single point of failure, provided a peripheral is cross-strapped. 


\subsubsection{Artificial Cell Implementation}

Figure 7.10 depicts the design of the multicellular testbed artificial cell. It can be compared to the reference Satellite Stem Cell design in Figure 4.6 of Chapter 4.

Each testbed cell contains four proteins. This number was reached based on the calculated maximum number of proteins which could fit within the CubeSat standard PCB dimensions of $10 \times 10 \mathrm{~cm}$. In addition, each cell contains a central, non-volatile 'DNA' memory IC, a discrete watchdog IC and a CAN bus bridge.

Each protein is composed of five elements: a microcontroller with embedded bootloader and CAN hardware, an external CAN controller and transceiver IC, a set of power switches, an 'I2C I/O expander' IC, and a set of generic I/O channels.

The MCU is an NXP LPC11C24 ARM Cortex M0 which can be clocked at up to $50 \mathrm{MHz}$. It has $32 \mathrm{kB}$ of flash memory for program storage and $8 \mathrm{kB}$ of RAM. This MCU was chosen for several reasons. Firstly, the MCU needs to be physically small and consume as little power as possible, while having enough processing power to perform demanding tasks such as attitude estimation. The ARM Cortex M0 family, which was essentially designed to compete with 8-bit MCUs, is a good fit. The LPC11C24 comes in a small 7 x $7 \mathrm{~mm}$, 48-pin LQFP package and consumes less than $9 \mathrm{~mA}$, while providing 0.84 DMIPS/MHz of processing power (for a comparison of processing power, see the case study in Section 7.3). Secondly, the MCU has an embedded CAN controller and transceiver which saves board space. Furthermore, the MCU has a set of drivers for the CAN hardware in ROM, and, even better, a CAN-based bootloader. Using this bootloader, new firmware can be uploaded to the MCU over the CAN bus. Finally, NXP provides very inexpensive hardware development kits for the LPC11C24, which come with a licence for their Eclipse-based development environment. A number of other MCUs, such as the Texas Instruments MSP430, would make suitable alternatives, provided the CAN transceiver is provided and a bootloader written.

The internal CAN transceiver is used to communicate over the inter-protein bus. This bus, which is also shown in Figure 7.10, is the first of three buses interconnecting the proteins on a cell. Any protein on a cell can send messages on this bus to other proteins on the same cell, including the MM. Thanks to the LPC11C24's CAN bootloader, the same bus is used by the MM to reprogram proteins. The second bus present on every cell is the inter-cellular bus. It is also based on the CAN standard but, under normal operations, is only read from and written to by the MM protein. However, since every protein has the ability to become the MM, all proteins must at least be electrically interfaced to the bus. This is achieved through an external CAN controller and transceiver IC, the MCP25625. This solution allows the 


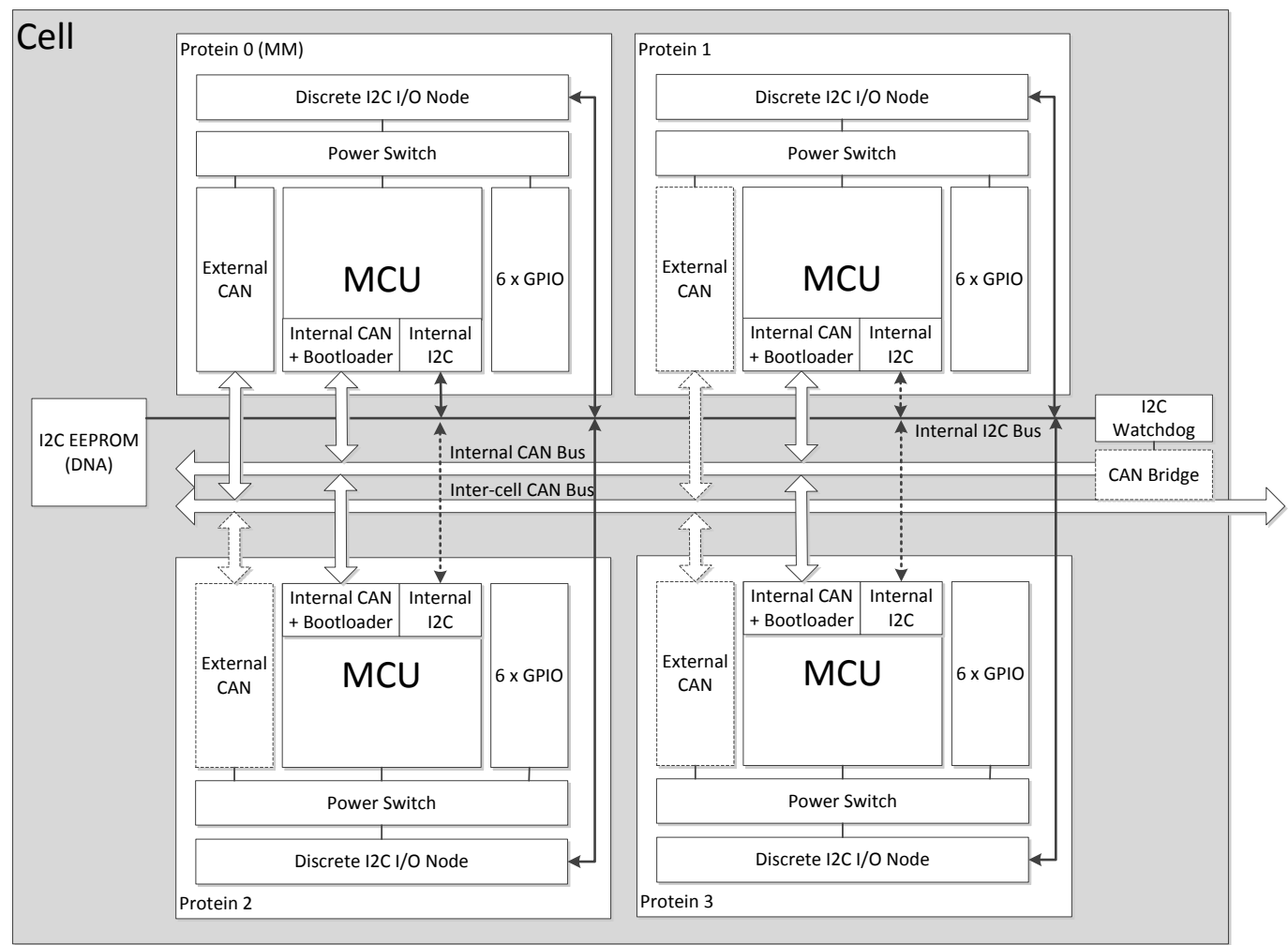

Figure 7.10: Implemented cell architecture based on four proteins interconnected by three buses.

power to the MCP25625 to be removed on non-MM proteins (putting the MCP25625 in a supported, high-impedance state), mitigating the risk of bus interference.

Removing power to the MCP25625 is achieved with a MOSFET switch controlled via an I2C I/O expander. The I2C I/O expander is a simple device which provides a number of digital inputs and outputs which can be controlled via I2C. In addition to providing control over the MCP25625 power, it also provides individual power control for the MCU and generic I/O channels, monitors for MCU over-current conditions, and can place the MCU into bootloader mode via dedicated MCU pins. An I2C bus, therefore, is the third bus which spans the cell. It is a single-master bus with the MM acting as master. The $\mathrm{I} 2 \mathrm{C}$ bus also provides access to the 'DNA' non-volatile memory, which in this case is implemented using a pair of $1 \mathrm{Mbit}$ I2C Ferroelectric RAMs (FRAMs). These provide storage space for firmware images and runtime variables. Thus, the process an MM follows to reprogram one of its proteins follows these steps:

1. MM pulls the protein MCU's bootloader pin low via the I2C bus and I/O expander

2. MM cycles power to the protein via the $\mathrm{I} 2 \mathrm{C}$ bus and $\mathrm{I} / \mathrm{O}$ expander 
3. MM checks that the protein is in bootloader mode by pinging it over the inter-protein CAN bus

4. MM reads new firmware image from the FRAM via $\mathrm{I} 2 \mathrm{C}$ bus and transfers it the to protein over the inter-protein CAN bus

5. Protein's bootloader writes the new firmware to flash memory

6. MM releases bootloader pin and power cycles protein as before

A final purpose of the $\mathrm{I} 2 \mathrm{C}$ bus is to provide the interface to a discrete real-time clock and watchdog IC. The MM is required to prove it is still alive by periodically 'kicking' the watchdog. If the watchdog times out it will trigger two events. Firstly, it will activate a CAN bridge between the internal and external CAN buses. The CAN bridge is a discrete IC which, when activated, transparaently forwards all traffic between the two separate CAN buses, effectively merging them into a single bus. The CAN bridge allows proteins on the cell to continue interacting with the rest of the system despite the absence of the MM router task. The bridge also allows the MM on another cell to reprogram proteins on the stricken cell. In additional to activating the $\mathrm{CAN}$ bridge, a watchdog timeout also triggers a hardware interrupt in all of the cell's proteins by pulling a dedicated signal line low. As will be described in more detail in Section 7.2.3, this causes one of the proteins on the cell to enter bootloader mode, ready to be reprogrammed into the new cell $\mathrm{MM}$. This process follows these steps:

1. Another cell in the system senses the bootloader-mode protein on the inter-cellular CAN bus. This signals the loss of an MM on a system cell.

2. The 'saviour' cell's MM reads the MM firmware from FRAM and transfers it between cells (via the inter-cellular bus and CAN bridge) to the bootloader-mode protein.

3. Bootloader writes the new firmware to flash memory

4. Saviour MM resets the bootloader-mode protein using a CAN command

5. Bootloader-mode protein restarts as the formally-stricken cell's new MM.

6. New MM resets the watchdog, which disables the CAN bridge, and continues as normal

The final component of each protein is the generic I/O circuitry. Each protein has six generic I/O channels which are electrically identical to the prototype I/O channels, but have had their layout optimised. Originally, eight I/O channels per protein were planned to match the requirements of Table 4.1 in Chapter 4 . However, during layout it became apparent that 


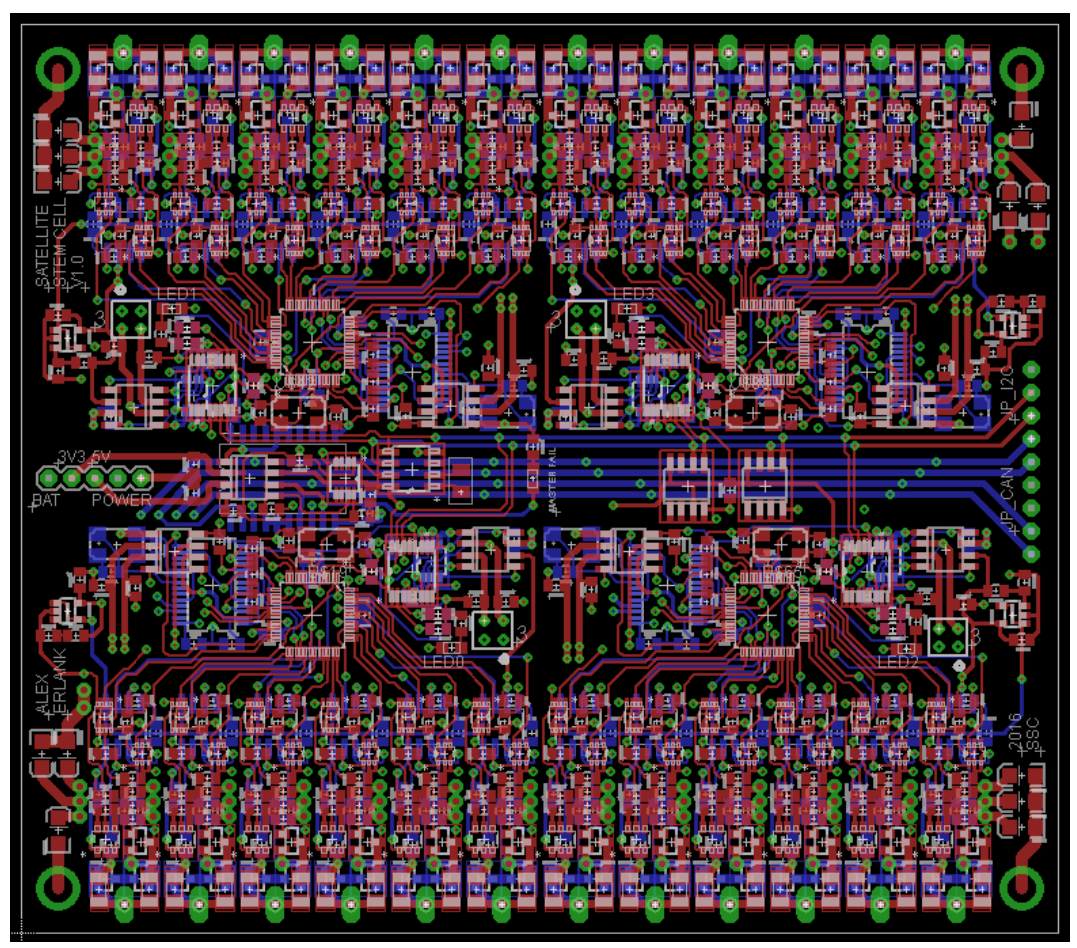

Figure 7.11: Implemented artificial cell PCB layout. Notice the repetition of the generic I/O circuitry, and the symmetry of the four proteins.

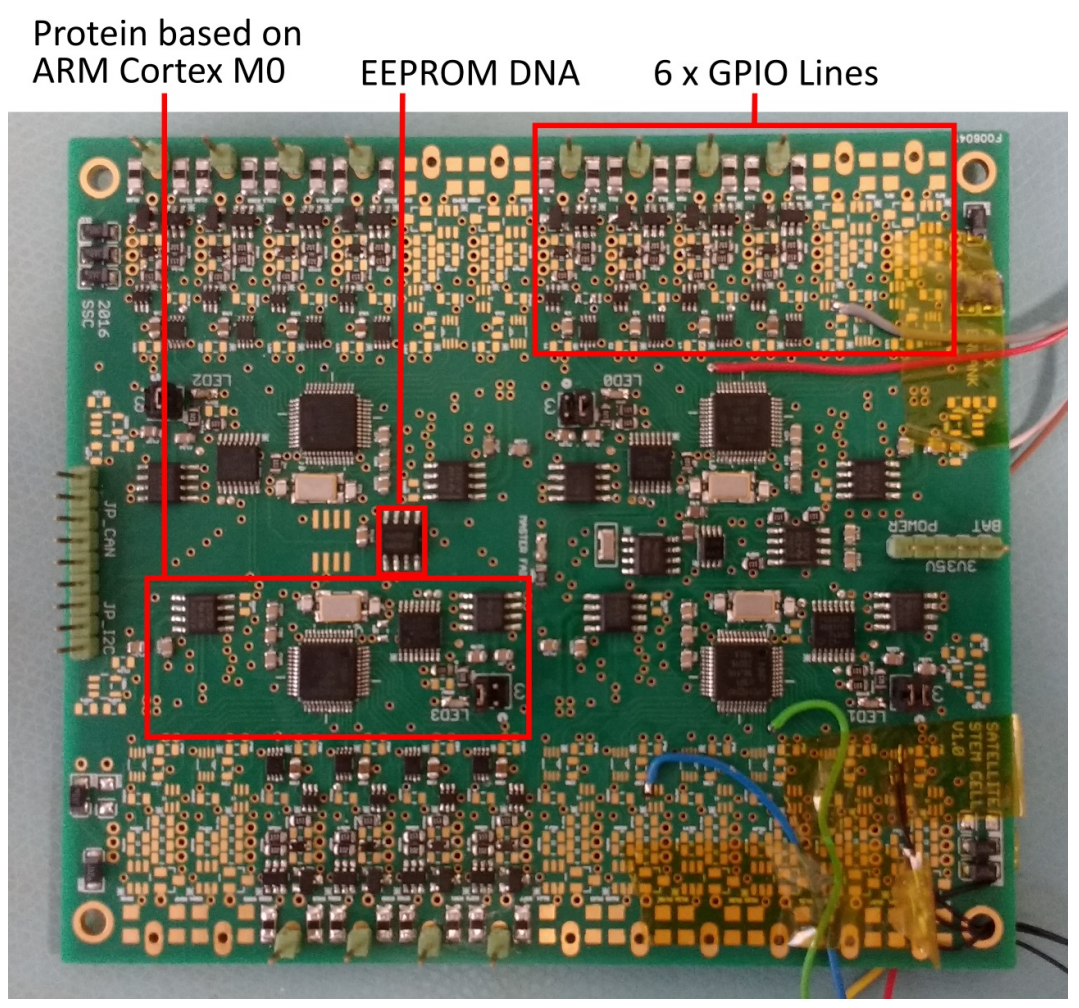

Figure 7.12: Completed testbed cell (barring a few I/O channels), with important sections highlighted. 
the number would have to be reduced to fit into the space available. Nevertheless, with six channels the majority CubeSat peripherals can be interfaced to. The complexity of the generic I/O layout can be seen in Figure 7.11, which shows the layout of two signal layers of the four-layer cell PCB. A complete (barring a few I/O channels) testbed cell is shown in Figure 7.12, with important sections highlighted.

\subsubsection{HARP Middleware Implementation}

The HARP middleware implementation is composed of two parts, namely, the MM firmware and an agent template. Both are based on the official FreeRTOS V7.1.0 port for the LPC1114 MCU, which is a close sibling of the LPC11C24 [100]. FreeRTOS is a free, open-source, real-time operating system for embedded environments. It has been used successfully on board several low-cost satellites, including the CubeSats STRAND-1 [101], Alsat-1N [102] and ESTCube-1 [103], and ports are available for many architectures.

The MM firmware implements the AMS, TAE and Router agency services, as well as other housekeeping tasks, as a set of four FreeRTOS threads (excluding the idle thread). The threads have hard-coded priorities, with the Router receiving the highest priority and the TAE receiving the lowest. The full set of threads, their priorities and their required stack space are given in Table 7.2.

The complete MM firmware, including FreeRTOS port, agency services and relevant hardware drivers, compiles to $28.7 \mathrm{kB}$ in NXP's LPCXpresso development environment with compiler size optimisation enabled. This consumes approximately $90 \%$ of the LPC11C24's flash memory.

A comparison of the memory requirements of the embedded agent platforms JADE-LEAP, Mobile-C and Agilla, is given in Table 7.3. As these values were generated under different conditions, they can only be used as a rough, order-of-magnitude comparison. However, it is evident that HARP's memory footprint is orders of magnitude smaller than traditional agent platforms (and comparable to Agilla).

Due to the limited RAM ( $8 \mathrm{kB})$ available in LPC11C24 MCUs, some constraints had to be placed on ACL messages. Firstly, agent names, which are represented as character arrays, are limited to two bytes in length. Thus, the agency goes by the name 'MM' while agents have names such as 'A1' or 'RW'. Secondly, ACL message contents are limited to 11 bytes in length. Both of these limitations could easily be lifted if the middleware was ported to a system based on protein MCUs with more RAM. 
Table 7.2: The priorities, responsibilities and stack sizes of the FreeRTOS threads composing the HARP agency middleware.

\begin{tabular}{|l|l|l|l|}
\hline Thread & Priority & Implements & Stack Size (Bytes) \\
\hline 1 & lowest & Health check & 1792 \\
\hline 2 & medium & AMS & 1712 \\
\hline 3 & medium & $\begin{array}{l}\text { Kick watchdog } \\
\text { Broadcast agent list }\end{array}$ & 624 \\
\hline 4 & high & Router & 544 \\
\hline
\end{tabular}

Table 7.3: Order of magnitude comparison of the memory requirements of agent platforms designed for embedded applications [81][80].

\begin{tabular}{|l|l|l|}
\hline Agent Platform & $\begin{array}{l}\text { ROM Requirement } \\
(\mathbf{k B})\end{array}$ & $\begin{array}{l}\text { RAM Requirement } \\
(\mathbf{k B})\end{array}$ \\
\hline JADE-LEAP & 17782 & 600 \\
\hline Mobile-C (emb. Ch Interpreter) & $\sim 3000$ & \\
\hline HARP & 28.7 & 8 \\
\hline Agilla & 57 & 3.3 \\
\hline
\end{tabular}

The agent template, which forms the second half of the HARP middleware, provides a FreeRTOS thread for handling ACL communication and a library for utilising the generic I/O hardware. Additionally, the agent template provides the routines for handling the MM-failure hardware interrupt. This interrupt occurs when MM failure has caused the cell's watchdog to time-out. Upon detecting this condition, the thread will:

1. Broadcast a CAN message, including the priority of its current task, on the inter-protein bus stating it has received the interrupt.

2. Wait $500 \mathrm{~ms}$ and listen for similar messages from other proteins on the cell.

3. If it determines that it has the lowest priority task, enter bootloader mode, otherwise clear interrupt and continue.

At this point the protein which has entered bootloader mode will be reprogrammed into the new cell MM by another cell in the system.

The agent template compiles to $24.9 \mathrm{kB}$, or $78 \%$ of an LPC11C24's flash memory. While the remaining space for user code is limited, the provided threads and libraries should allow the developer to focus on high-level functionality. Alternatively, agents need not be based on the agent template, or even FreeRTOS. As long as an agent is capable of registering with the AMS and responding to health checks, it can be deployed on the system. However, such 


\section{Multicellular System Implementations}

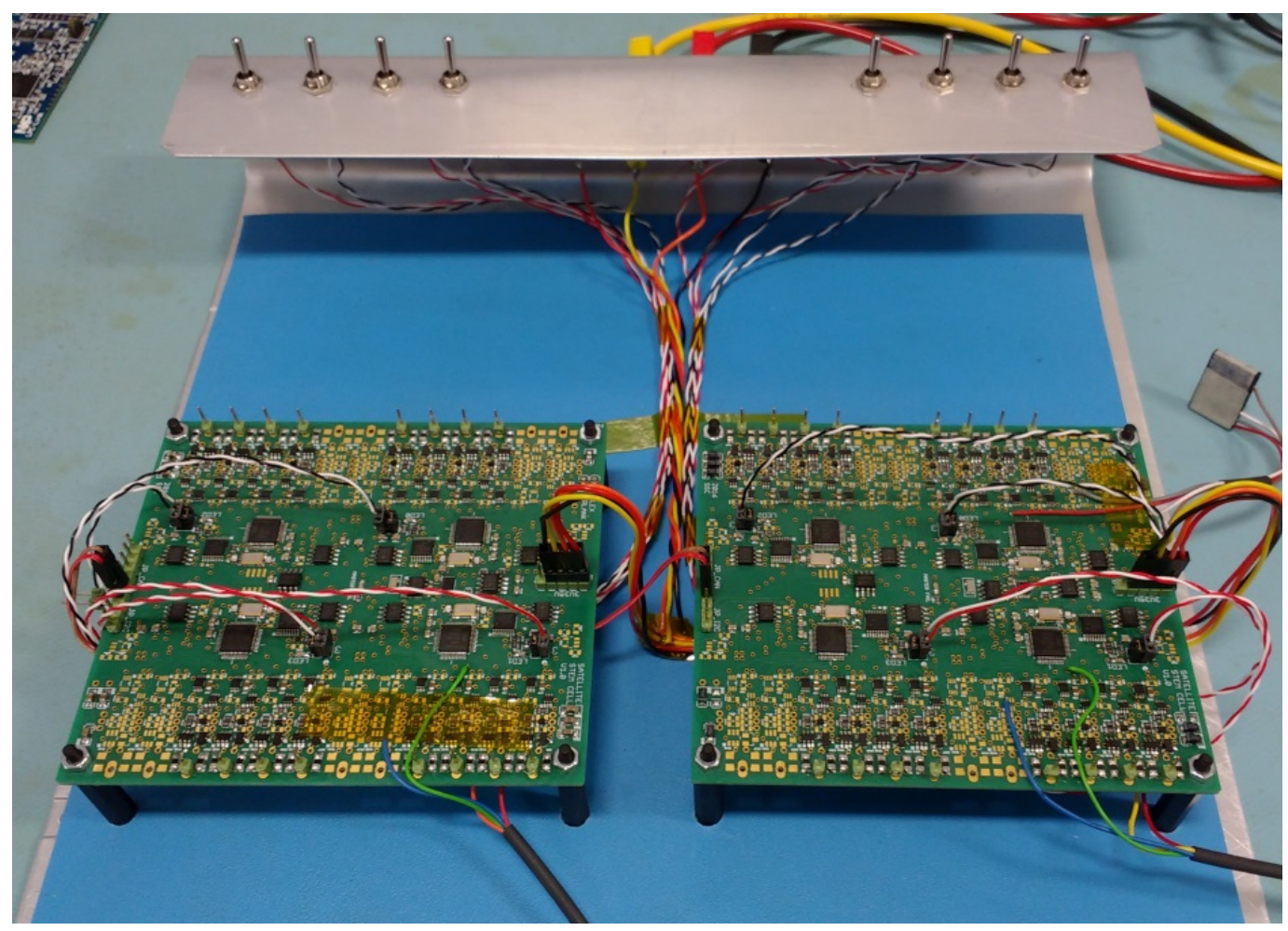

Figure 7.13: The complete multicellular testbed, composed of two cells. The toggle switches allow individual proteins to be switched off permanantly or momentarily, simulating failures. Both the inter-protein and inter-cellular bus traffic can be monitored through a terminal.

agents are not recommended as they are not necessarily capable of detecting and correctly responding to MM failures, which lowers the overall available redundancy.

\subsubsection{Demonstrating Functionality}

Figure 7.13 shows the completed multicellular system testbed. It is composed of two cells and includes a number of toggle switches for simulating permanent or momentary protein failures. Additionally, the inter-protein and inter-cellular bus traffic can be parsed and monitored on a terminal. For the majority of experiments, multi-colour LEDs were used as the peripherals, as they offer a convenient way of identifying an executing agent. Described in the subsections that follow are a series of demonstrations which were carried out to verify the full functionality of the multicellular testbed. In addition, further system implementation details are given to describe various observed and tested-for behaviours. 


\subsubsection{Task Allocation Demonstration}

The first demonstration consisted of three agents being deployed onto the system of two cells. A separate multicolour LED was attached as the peripheral for each of the eight proteins over the two cells. Each LED has four connections, namely common ground, red anode, green anode, and blue anode, which interfaced to four generic I/O channels. Each of the three agents flashed its LED a different colour when executing, allowing the agent to be easily identified. In addition, the MM identified itself by flashing its attached LED in white.

The demonstration began with the two cells containing only MMs. As time progressed, the MMs began deploying the agents onto their proteins. It takes approximately 10 seconds for an MM to program a protein with new agent firmware. Once all three agents were executing, as verified by three peripheral LEDs flashing red, green, and blue, respectively, the MMs continued by deploying cold redundant versions of the agents onto the remaining proteins.

The task allocation strategy was tested by severing the inter-cellular bus between the two cells. At this point, each of the three agents began executing on each of the cells, as each cell wrongly believed that no agents were executing anywhere else in the system. Reestablishing the inter-cellular link promptly (within 3 seconds) caused the duplicate agents to stop executing and become cold redundant.

The task allocation strategy was further tested by using the toggle switches to cause protein failures. Removing the power to an individual protein which was executing an agent causes the agent to reappear on another protein, which may or may not be on the same cell. This is only true if spare proteins are available, or the lost agent is of a higher priority than another agent in the system. In the latter case, the MM will reprogram one of its agents which are of a lower priority into the lost agent. Such an event has the potential to cause a cascade of reprogramming events, but the system always settles into a state which includes the highest priority agents. Additionally, an improved recovery time was noted for agents which could be recovered from their cold-redundant counterparts, compared to those which had to be reprogrammed. Cold redundant protein recovery takes less than a second.

Temporary failures were also introduced to test the system. It was demonstrated that the effect of temporarily disabling a protein is dependent on the duration of the failure. Very short interruptions, on the order of hundreds of milliseconds, could go undetected by the health checks and would simply result in the agent successfully re-registering with its AMS. Longer interruptions would trigger the system to begin redeploying the lost agent onto another protein. If the failed protein recovered before the new one was operational, the new 
agent's AMS registration would fail and it would be made cold redundant. On the other hand, if the failed protein only recovered after the new agent was operational, the original agent's attempt to re-register with its AMS would fail, and it would become cold redundant.

Finally, the loss of an MM protein was initiated. As expected, after the cell watchdog timed out after a few seconds, the CAN bridge was enabled and one of the remaining functional proteins ceased executing its agent and entered bootloader mode. The remaining proteins continued execution unaffected. The MM on the second cell successfully noticed the appearance of a bootloader-state protein on the inter-cellular bus and reprogrammed it into a new MM. Once the new MM booted, the CAN bridge was disabled and the system settled back into a steady state containing all three agents.

A commentated video of this demonstration in available online at [104].

\subsubsection{Continuity of Runtime Variables Demonstration}

The second demonstration aimed to test the saving and restoring of agent runtime variables. Both cells of the multicellular testbed were employed, but only a single agent was deployed onto the system. This agent flashed its peripheral LED through a predetermined sequence of colours. The current state of the sequence was stored in a runtime variable, which the agent requested the MM to store after every transition. Each store sequence followed the FIPA Request interaction protocol and resulted in the MM storing the runtime variable in the cell's non-volatile memory.

To test the continuity of runtime variables, a note was made of the current LED colour sequence state, before the protein on which the agent was running was deactivated using the toggle switches. At this point the system successfully redeployed the agent on another protein, from where it continued its sequence in the correct state. To do this, the agent must complete two steps upon starting execution. Firstly, it must use a FIPA request to register with its AMS. Secondly, it must use a FIPA query to ask for any relevant, stored, runtime variables. If such variables exist, as they did in this case, the MM will respond with the appropriate data. The agent can then use this data as a state checkpoint from which to continue executing. The choices of which runtime variables to store, how often to store them, and how to continue once runtime variables have been recovered, are up to the agent developer.

This sequence of events was tested several times to verify that runtime variables could also be transferred between cells. As explained in Chapter 5, every MM builds up a list of all agents currently executing in the system by listening out for agent directory broadcasts. When 
the Task Allocation Entity within an MM notices that an agent has failed (because it has not recently received notification of that agent's presence from any of the agent directory broadcasts), it notes the last cell on which the agent had been executing. As part of the process of redeploying the failed agent onto one of its own proteins, as part of the global task allocation strategy, it will query the previous host cell for any relevant runtime variable data. Thus, once the agent starts executing on the new cell, a copy of its runtime variables are available locally.

There are two common cases in which the MM will not service valid queries for runtime variables. The first case occurs when an agent is executed for the first time on the system. The second case occurs when the requested runtime variables have expired. In addition to deciding which runtime variables to store and how often to store them, the agent developer must also assign a longevity to each runtime variable. The longevity determines how long the data in a runtime variable is relevant for. For example, certain attitude control variables are updated every second and therefore expire after a second. On the other hand, some variables, such as temperature, change more slowly and can therefore stay relevant for longer periods. When the MM receives a query for stored runtime variables, it checks the save time and expiry time against its real-time clock. Expired variables are not returned, resulting instead in a query failure response. In either case, a failed query for runtime variables requires the agent to initialise from a default state.

The expiry of runtime variables was tested in the demonstration, too. This was done by leaving the agent's protein running, while deactivating all the rest. In this configuration, when the agent's protein was deactivated, too, there were no available proteins onto which the agent could be redeployed by the system. The system was left in this state until the LED-colour runtime variable should have expired (10 seconds in this case). At this point a random protein was reactivated and the agent was seen to restart on it. As expected, the agent had to start from the beginning of its colour sequence.

A commentated video of this demonstration is available on online at [105].

\subsubsection{Cross-Strapping Demonstration}

The third demonstration aimed to test peripheral cross-strapping. Only a single cell and a single were used. Instead of a multicolour LED, the peripheral in this case was an $\mathrm{I} 2 \mathrm{C}$ temperature sensor. Interfacing to the sensor requires four generic I/O channels: I2C data, I2C clock, 3V3 power, and ground. An LED was connected in parallel across the power and 
ground channels to give a quick indication that the sensor was receiving power. The single I2C temperature sensor was cross-strapped between two proteins.

To test the cross-strapping an agent was written which would periodically read the I2C sensor and report the temperature over the inter-cellular bus. The two proteins to which the I2C sensor was cross-strapped were enabled, while the rest were disabled. The system quickly deployed the agent onto one of the proteins, while the other was automatically programmed into a cold redundant spare. The active agent successfully read the sensor and reported the temperature every second. When the active agent's protein was physically disabled, simulating a permanent failure, the cold-redundant spare was activated by the system and successfully read from the same temperature sensor. This implies that the generic I/O circuitry of the 'failed' protein correctly entered high-impedance mode.

A commentated video of this demonstration is available online at [106].

A more thorough test of the peripheral interfaces would involve multiple peripherals crossstrapped in various configurations, including between cells. In this way, the task allocation strategy would have to take into account the reconfiguration limitations caused by the peripheral interfaces. This functionality is currently not implemented, but would be simple to do. Along with each task in the prioritised task list would be information indicating any required peripherals. Additionally, each cell would require a list in non-volatile memory of its current peripheral interfaces. With these two pieces of information, the Task Allocation Entity would be able to deploy agents requiring specific peripherals to proteins with those interfaces.

\subsubsection{Performance and Scalability Benchmarking}

In addition to functionality experiments, performance and benchmarking experiments were performed for determining overheads and scaling potential. In addition to proving practical feasibility, these characteristics are important for comparison to the conceptual multicellular satellite requirements derived in Section 3.1.4.

\subsubsection{Processing Capacity and Power Consumption}

The protein MCUs are based on ARM Cortex M0s which can be clocked at up to $48 \mathrm{MHz}$. The ARM Cortex M0 core is capable of 0.84 Dhrystone MIPS (DMIPS) per megahertz [107]. The Dhrystone benchmark is a popular test of processor performance and is based on a loop 


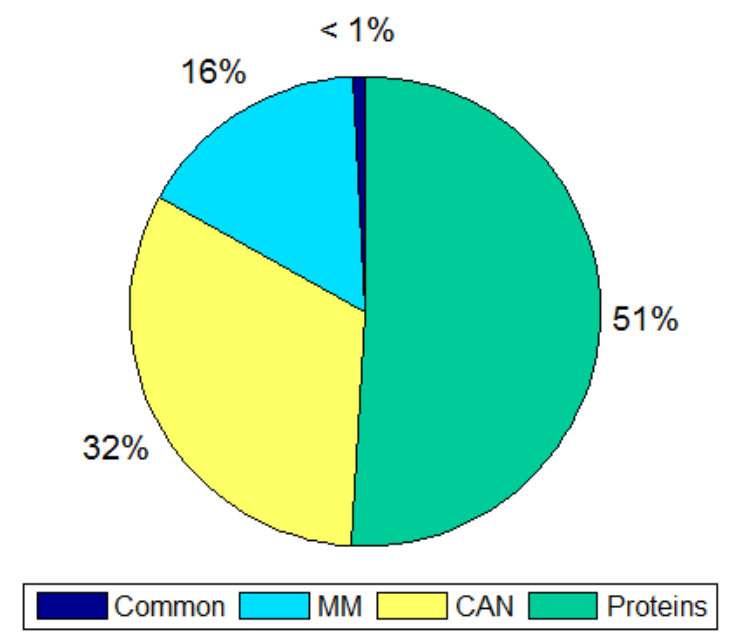

Figure 7.14: Power consumption breakdown of a prototype artificial cell from the multicellular testbed.

containing a number of 'representative' $C$ statements. The DMIPS unit is calculated by dividing the total number of iterations of the loop executed by the processor in one second, by the number achieved by the DEC VAX 11/780 minicomputer of the 1970s. Thus, with all four proteins clocked at $48 \mathrm{MHz}$, a cell has a total computational capacity of 161.28 DMIPS. However, one of the proteins is reserved for the MM, so a total of 120.96 DMIPS is available to the developer.

At this level of performance, a cell consumes $390 \mathrm{~mW}$. The power consumption breakdown can be seen in Figure 7.14. $32 \%$ of the power consumption is attributable to the operation of the two CAN buses. Operating at $5 \mathrm{~V}$, they consume significantly more power than the common CubeSat I2C bus. With only four proteins on the cell, the MM power consumption overhead, at $16 \%$, is also substantial. Increasing the number of proteins per cell will reduce this overhead. However, as will be shown in Section 7.2.5.3, the maximum number of proteins per cell is limited by the finite processing capacity of the MM.

The power consumption can be lowered by reducing the clock speed of user proteins to $12 \mathrm{MHz}$, giving a total processing capacity of 30.24 DMIPS. At this performance, power consumption is reduced by $25.8 \%$ to $310 \mathrm{~mW}$.

A comparison of this performance and power consumption with respect to other satellite platforms is given in the case studies of Section 7.3. 


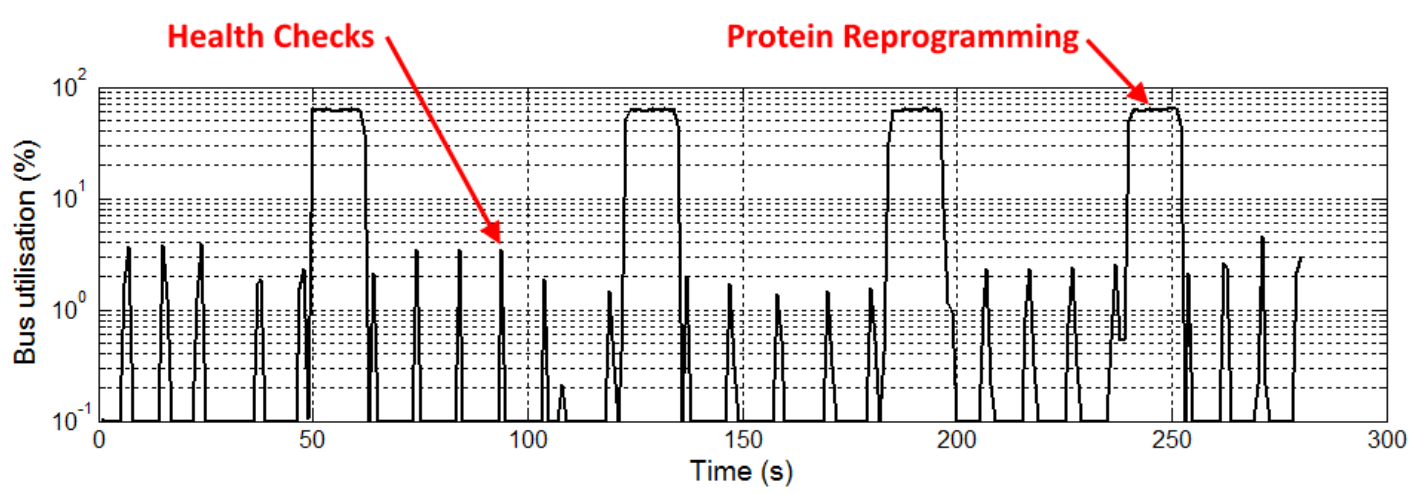

Figure 7.15: Inter-protein CAN bus bandwidth utilisation by routine MM health checks and reprogramming procedures.

\subsubsection{Housekeeping Bus Overheads}

Housekeeping activities central to the ASC Architecture, such as protein health checks and protein reprogramming, come with overheads. The health check routine makes use of FIPA ACL messages and interaction protocols. Despite the bit-efficient encoding of ACL messages, ACL messaging incurs a large bandwidth overhead. A typical ACL message is 70 bytes long and can contain a maximum of 11 message payload bytes (a limitation of the HARP implementation). Furthermore, every ACL message forms part of an interaction protocol, which involves the exchanges of at least two ACL messages. Thus, transferring 11 bytes of message data from one agent to another results in at least 140 bytes of transfer layer traffic. At the ISO-TP layer, this will translate into 22 CAN frames (1 start frame, 1 flow control frame, and 20 consecutive frames). Finally, 22 CAN frames require 297 bytes ( 2376 bits) of physical link traffic. Thus, in this implementation, ACL messaging is only envisioned to be used for low bandwidth data exchanges and for setting up subscriber-consumer relationships based on real-time CAN messages. Future enhancements to the HARP middleware should include a substantial increase to the maximum ACL message content length, to lower these overheads.

The protein reprogramming procedure does not use ACL messaging and instead uses CAN frames following the CanOpen protocol, as specified by the LPC11C24's CAN bootloader. Thus, the reprogramming procedure has far less overhead, but nevertheless produces a large amount of data on the CAN bus. Due to the addressing scheme used, this data takes priority over ACL messages, but not over real-time CAN traffic. It is important for the programming to complete quickly, as it represents a period of increased risk from radiation induced faults. 
Figure 7.15 shows the inter-protein CAN bus bandwidth overhead caused by the MM's health check routine, and by the protein reprogramming process. During this experiment the CAN bus was operating at $100 \mathrm{~kb} / \mathrm{s}$. Protein failures were induced using the toggle switches at 40 and 110 seconds, leading to the large peaks in bus utilisation as reprogramming occurred. The failed proteins were revived at 180 and 240 seconds, leading to two more automatic reprogramming sessions. The reprogramming process can consume up to $65 \%$ of the bus bandwidth.

The smaller peaks in between programming sessions are caused by the MM's periodic, ACLbased, protein health checks. Each health check consists of a single ACL query interaction per protein, and therefore creates approximately $10 \mathrm{~kb}$ (2376 bits x 4 proteins) of CAN traffic. Therefore, the theoretical maximum health check rate for the prototype cells is $10 \mathrm{~Hz}$ over the $100 \mathrm{~kb} / \mathrm{s}$ bus. However, in practice, a health check rate at least one order of magnitude slower should be used to avoid causing excessive bus traffic. In the experiment of Figure 7.15 a rate of $0.1 \mathrm{~Hz}$ was implemented. The height of the peaks caused by the health checks is seen to fluctuate over time, and is caused by the varying numbers of active proteins during the experiment. However, the health check routine never consumes more than $4 \%$ of the bus bandwidth.

System recovery time after a protein failure is dependent on several variables, including the failure type, health check rate, current system redundancy, agent size, and number of stored runtime variables. However, given knowledge of these variables, the recovery time is deterministic. The experiment of Figure 7.15 shows that reprogramming takes approximately 10 seconds for a $32 \mathrm{kB}$ agent, while the complete recovery procedure, from fault detection to new agent execution start, can take up to 30 seconds with a $0.1 \mathrm{~Hz}$ health check rate. Thus, this system is not suitable for applications which require full fail-operational capabilities (although a certain level of operation will always be available). However, for a satellite in orbit such recovery times are generally tolerable, especially if it negates the need for a ground controller to intervene.

\subsubsection{AMS Performance and Router Bandwidth}

Beyond the limits imposed by the housekeeping overheads, several other factors limit the scaling potential of the system. Firstly, the 11-bit CAN bus addressing scheme limits the the total number of cells in the system to 15 , and allows a maximum of 126 proteins per cell. This limitation could be overcome by switching to extended addressing mode, which is supported by most CAN hardware and extends the address field to 29 bits. 
The second scaling limitation is imposed by the AMS transaction bandwidth. Since the AMS can only service a finite number of transactions per second, a cell with too many proteins may suffer from an overloaded AMS. As mentioned before, in addition to the health checks, common AMS interactions include requests to register or store runtime variables, and queries for protein address lookups. Experiments determined that each AMS of the test bench system is capable of processing a maximum of 20 AMS transactions per second.

Finally, the Router task, which is responsible for forwarding traffic between the inter-protein and inter-cellular CAN buses on each cell, also has a limited bandwidth. The Router Task has the highest priority of all the AMS services to ensure that real-time CAN traffic is always forwarded. Experiments determined that handling more than $500 \mathrm{CAN}$ frames per second results in the Router task starving the other tasks of processing time. Since all real-time CAN traffic is automatically forwarded into every cell (unlike ACL traffic which is filtered), the entire multicellular system must produce significantly less than 500 frames ( $32 \mathrm{kB}$ ) of real-time CAN data per second. Provided a separate network is implemented for handling payload data (often in the form of point to point links) and the attitude control bandwidth is limited to a few hertz, the available bandwidth should be sufficient.

\subsubsection{GPIO Cross-Strapping Limitations}

Ideally, it should be possible to cross-strap the same peripheral to an arbitry number of $\mathrm{I} / \mathrm{O}$ channels. However, due to parasitic capacitances and leakage currents, there are limits on cross-strapping. These limits vary by application. The addition of cross-strapping will have minimal affect on slow, high power signals, but will degrade the quality of analogue input and high-speed digital signals.

To understand the cross-strapping potential of the testbed I/O circuitry, an I2C peripheral was chosen as test case. Using $2.2 \mathrm{k} \Omega$ pull-up resistors, an I2C temperature sensor was interfaced to a single GPIO channel and transactions were successfully completed at $100 \mathrm{kbits} / \mathrm{s}$. The shape of the I2C clock waveform is shown in Figure 7.16. From this figure, the RC constant rise time can be remeasured to be approximately 1.5 us. After cross-strapping the same I2C temperature sensor to a second I/O channel and repeating the test, the time constant increased to approximately 2.5 microseconds, as shown in Figure 7.17. Thus, we can calculate that the time constant added by each additional cross-strapped I/O channel is approximately 1 us.

Based on this measurement, and the known $2.2 \mathrm{k} \Omega$ resistance, the I/O channel capacitance is calculated to be approximately $455 \mathrm{pF}$. Based on the $\mathrm{I} 2 \mathrm{C}$ specifications, the maximum 
a)

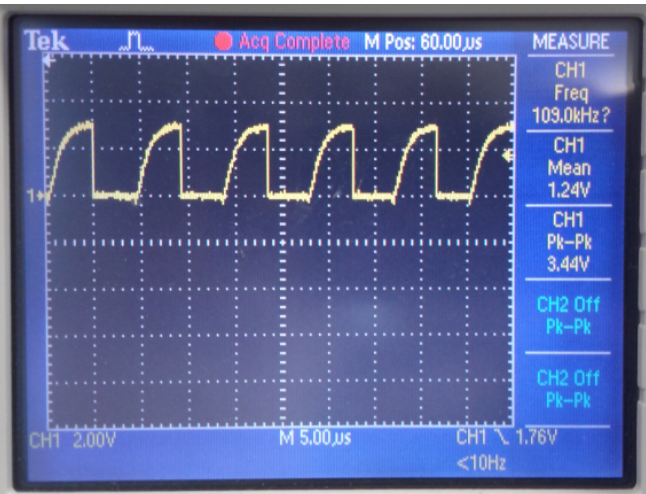

b)

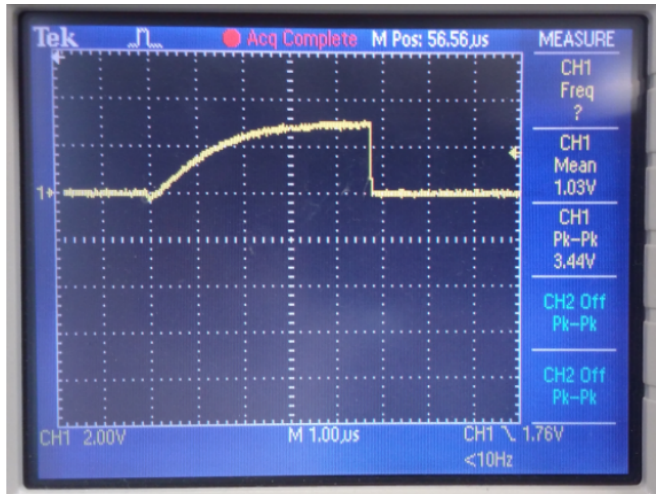

Figure 7.16: $100 \mathrm{~kb} / \mathrm{s}$ I2C clock waveform without cross-strapping (a), zoomed-in (b).

a)

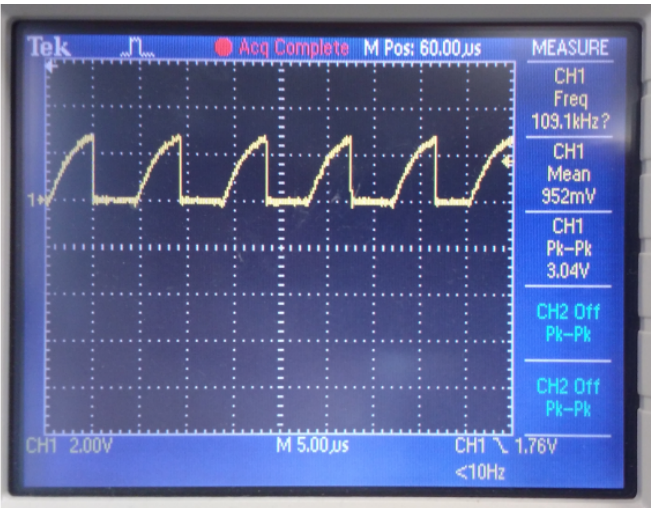

b)

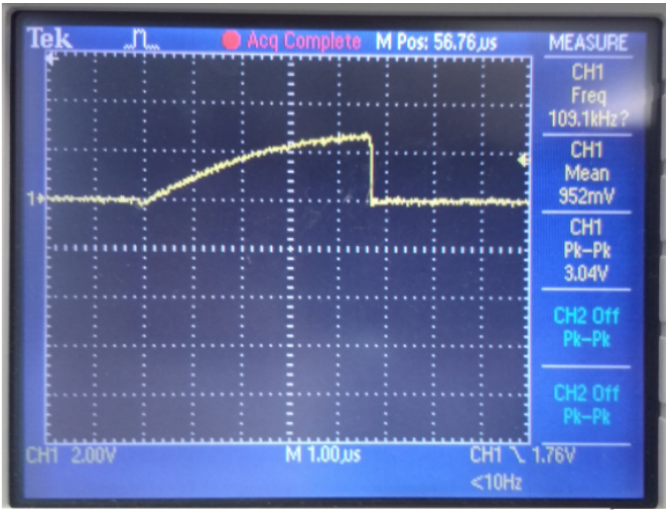

Figure 7.17: $100 \mathrm{~kb} / \mathrm{s}$ I2C clock waveform with cross-strapping between two I/O channels (a), zoomed-in (b). Note the increased rise time.

allowable I2C bus capacitance is $400 \mathrm{pF}$ [108]. Thus, despite working in practice, even a single $\mathrm{I} / \mathrm{O}$ channel violates this requirement.

The majority of this unwanted capacitance comes from the MOSFETs. For example, the output capacitance of the FDN371N MOSFET, which forms the lower switch in the I/O half-bridge, is given in the datasheet as $197 \mathrm{pF}$. The next generation of I/O circuitry will have to be designed with this parasitic capacitance in mind. However, even a brief survey of MOSFETs reveals that the current I/O circuitry could be updated with footprint-compatible MOSFETs with output capacitances below 30 pF (Si2308BDS).

Ultimately, the best performance could be achieved by building the I/O circuitry, either individually, or as a whole bank, within a single ASIC. 


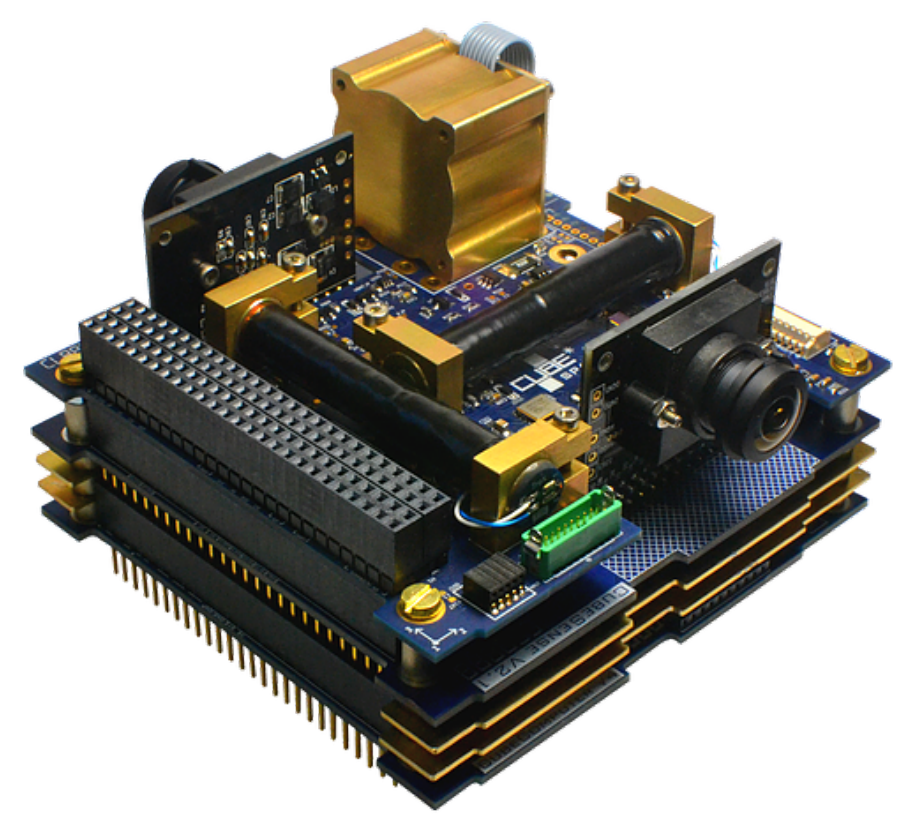

Figure 7.18: The CubeADCS, an integrated COTS CubeSat attitude determination and control system available from CubeSpace [109].

\subsection{Multicellular Satellite Case Studies}

To truly demonstrate the benefits and practical feasibility of the Artificial Stem Cell Architecture, two case studies are presented. In these case studies, the measured performance characteristics of the testbed cells are used to theoretically 'cellularise' two traditional sets of satellite avionics. Thereafter, the reliability, power consumption, volume and processing capacity of the cellularised and traditional systems are compared.

The first case study is based on a COTS, CubeSat-scale, ADCS subsystem. Its functionality is replicated using only 8 proteins. Nevertheless, the system is interesting because of its ability to operate in several different modes and its large number of interfaces to diverse sensors and actuators. The second case study represents a larger multicellular system, composed of approximately 40 proteins, and replicates the combined functionality of several microsatellite susystems.

\subsubsection{CubeSat ADCS}

The CubeSpace CubeADCS, shown in Figure 7.18, is an integrated CubeSat attitude estimation and control solution [109]. Attitude is estimated by an ARM Cortex M3 MCU using 


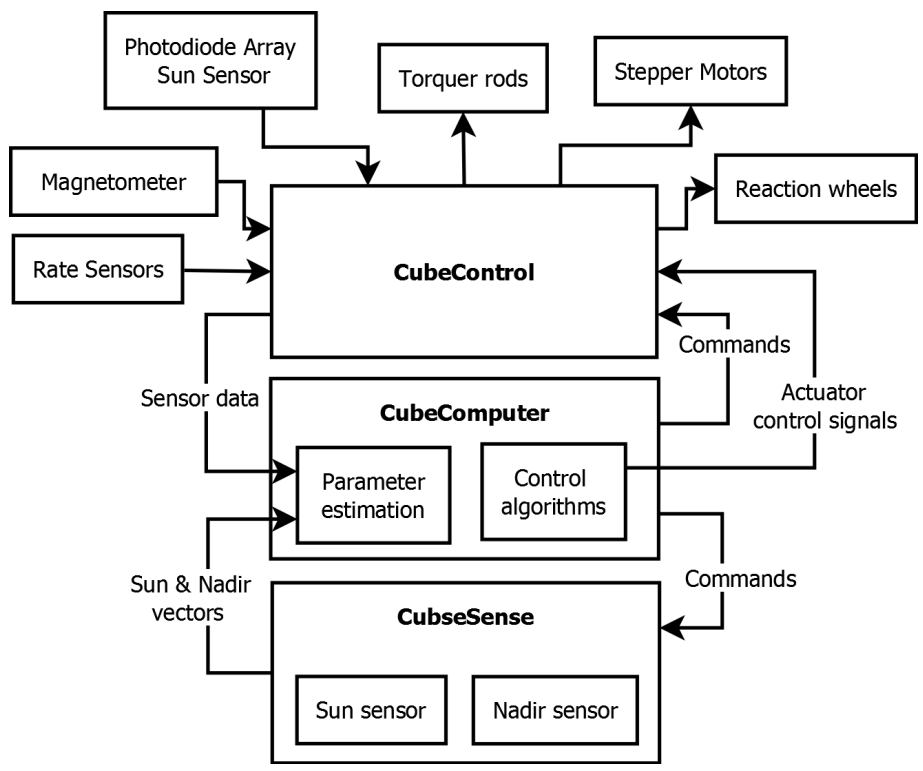

Figure 7.19: The architecture of the full-featured CubeADCS [112].

input from a magnetometer, coarse and fine sun sensors, a horizon sensor, and a gyroscope. Attitude is controlled by a set of three mangetorquers and one to three reaction wheels. The CubeADCS can operate in several modes, including detumble, pure magnetic control, and momentum-biased control. To date, the CubeADCS has flown on a number of SSC CubeSats and QB50 CubeSats [110] [111].

The most full-featured CubeADCS is composed of three distinct systems, named CubeComputer, CubeControl and CubeSense, existing as three separate $10 \mathrm{x} 10 \mathrm{~cm}$ PCBs in a stack. This architecture is shown in Figure 7.19. CubeComputer contains the main ARM Cortex M3 MCU, which handles telemetry and telecommands over an external I2C bus. Additionally, it executes attitude estimation and control loops and controls the other two systems over an internal $\mathrm{I} 2 \mathrm{C}$ bus. CubeControl provides an interface to the majority of the attitude sensors and actuators, while CubeSense, fitted with two CMOS cameras with fish-eye lenses, provides fine sun and nadir attitude vectors. For the purposes of this comparison a simpler version of the CubeADCS bundle is used. This version does not include CubeSense and contains only a single momentum wheel. Despite its reduced features, such a system is still capable of three-axis stabilising a satellite into a nadir-pointing attitude.

To cellularise a system, the discrete tasks it performs must be identified, together with required peripherals. The number of tasks then roughly determines the minimum number of proteins required. In this case, six ADCS tasks were identified, requiring two prototype cells to implement. The tasks are given priorities according to the operational modes in which they 


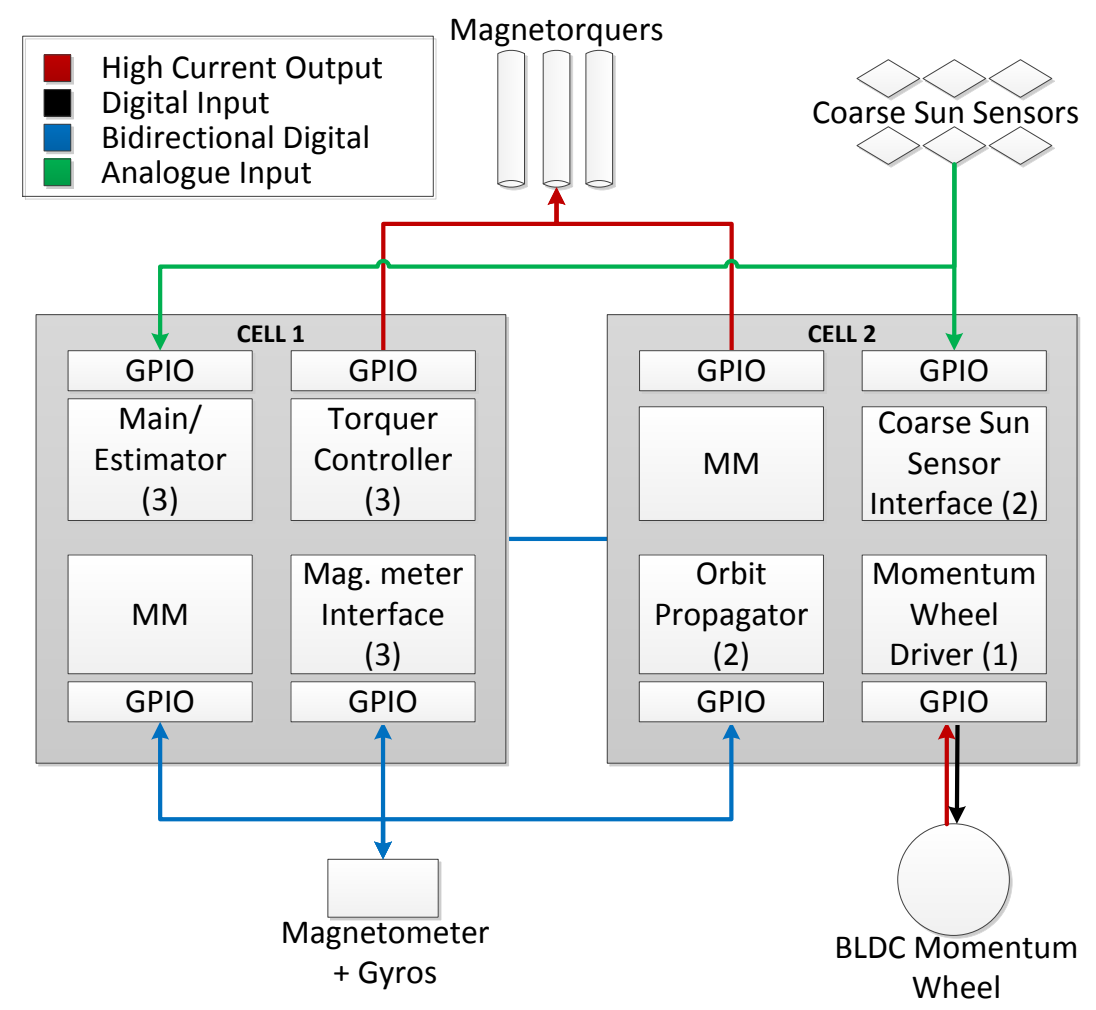

Figure 7.20: The architecture of a 'cellularised' CubeADCS, composed of two testbed cells and cross-strapped peripherals.

are required. The highest priority tasks are required for the most basic mode, which in this case is detumbling. These tasks include the magnetometer interface, magnetorquers driver, and main attitude control loop. The next mode, magnetic control, requires the addition of the coarse sun sensor interface and orbit propagator tasks. Finally, the most complex and capable mode, momentum-biased mode, requires the addition of the momentum wheel controller task. An example distribution of these tasks across the cells of a multicellular system is shown in Figure 7.20.

It is sensible to relate the level of cross-strapping of peripherals to their priorities. In this case, the magnetometer is triple-strapped (because it is essential for estimating attitude), while the magnetorquers and Sun sensors are double-strapped. The momentum wheel, because it is only required in the most complex mode, has only a single interface. 

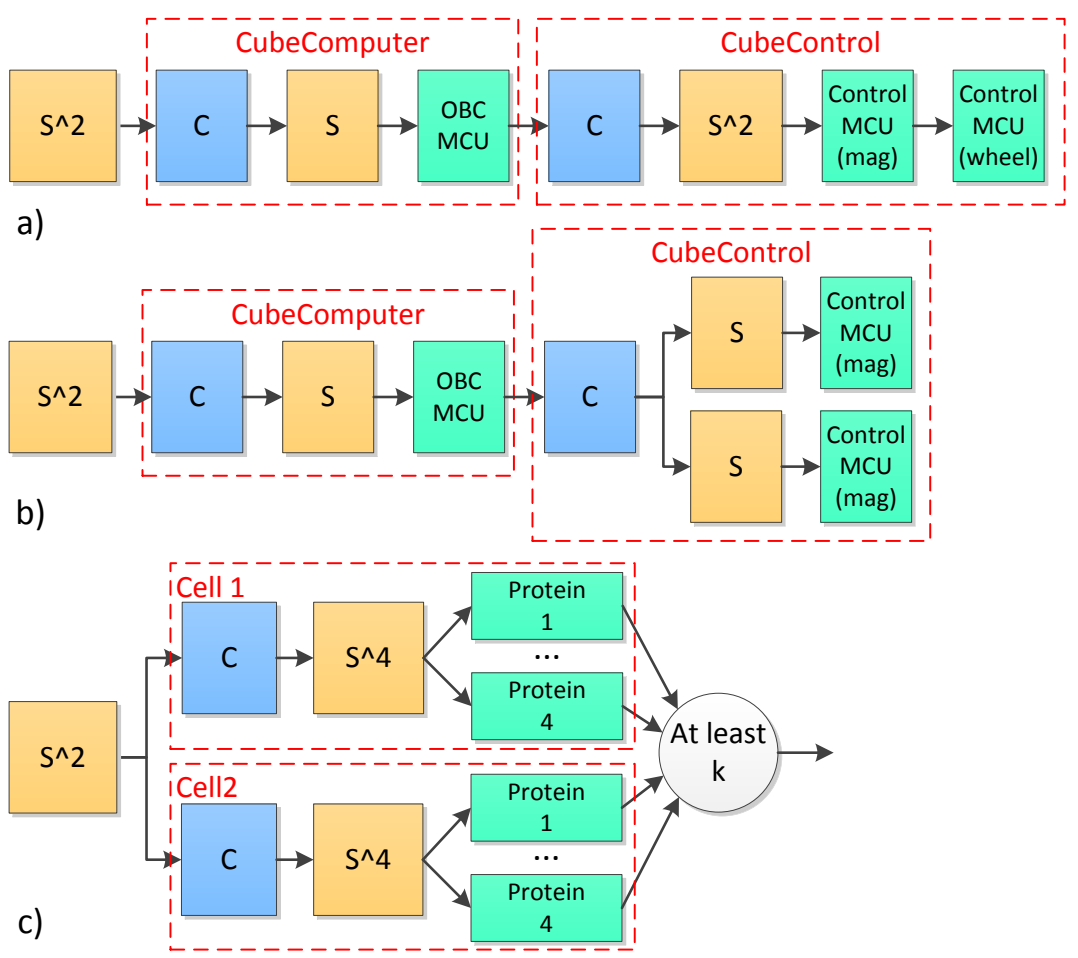

Figure 7.21: Relaibility block diagrams of the CubeADCS operating in momentum-biased mode (a), magetic control/detumbling mode (b), and the equivalent cell-based design (c). C - common, S support components. 


\subsubsection{Reliability Comparison}

Now that the cell-based ADCS is fully defined, it is possible to use the reliability analysis of Chapter 6 to compare its predicted reliability to that of the CubeADCS. In its most capable mode, momentum-biased mode, the CubeADCS is essentially a series system, as shown in Figure 7.21a. CubeControl contains two MCUs, one of which controls the momentum wheel, while the other handles the magnetorquers and magnetometer [112]. The common and support blocks represent support circuitry and buses on and between the PCBs of the CubeADCS stack.

In its two reduced-capability modes, detumble and magnetic control, the wheel is not required. Therefore, the MCU which normally controls the wheel has also been given the ability to operate the magnetometer and torquers as a form of redundancy. Thus, in these modes the block diagram appears as in Figure 7.21b, showing a partial parallel structure.

Based on these block diagrams of series and parallel architectures, the predicted reliability of the CubeADCS was analytically calculated. Failures are assumed to be independent and are modelled using Weibull distributions. A shape parameter of 0.4464 was derived from an empirical study of in-orbit satellite reliabilities [26]. The scale parameters for the MCUs, common and support blocks, of $60 e^{3}, 35 e^{8}$ and $35 e^{7}$ days, were adjusted to give the CubeADCS its expected design lifetime of one year, while roughly taking into account their relative complexities.

In contrast to the series and parallel architectures of the CubeADCS, the cell-based ADCS is described, as before, as a modified k-out-of-n architecture (Figure 7.21c). It is important to note that the representation of the system given in Figure 7.21c does not depict the requirement for an $\mathrm{MM}$ on each cell, or the restrictions imposed by peripheral interfaces. Thus, to more accurately model the system, the custom multicellular system simulator (described in Chapter 6) was employed. In each simulation the system begins in its most capable mode and transitions automatically into lower modes as failures build up. As described before, momentum-biased, magnetic control, and detumbling modes require a minimum of three, five and six user proteins receptively. To enable comparison, the same Weibull distributions used to model the CubeADCS were applied to the cell-based system.

The reliability plots of Figure 7.22a compare the predicted lifetimes of the CubeADCS and cell-based ADCS in various modes of operation. In detumble mode, the cell-based ADCS shows a reliability advantage throughout the mission lifetime. In magnetic control mode, the cell-based ADCS starts out more reliable, but falls below the CubeADCS within the first 

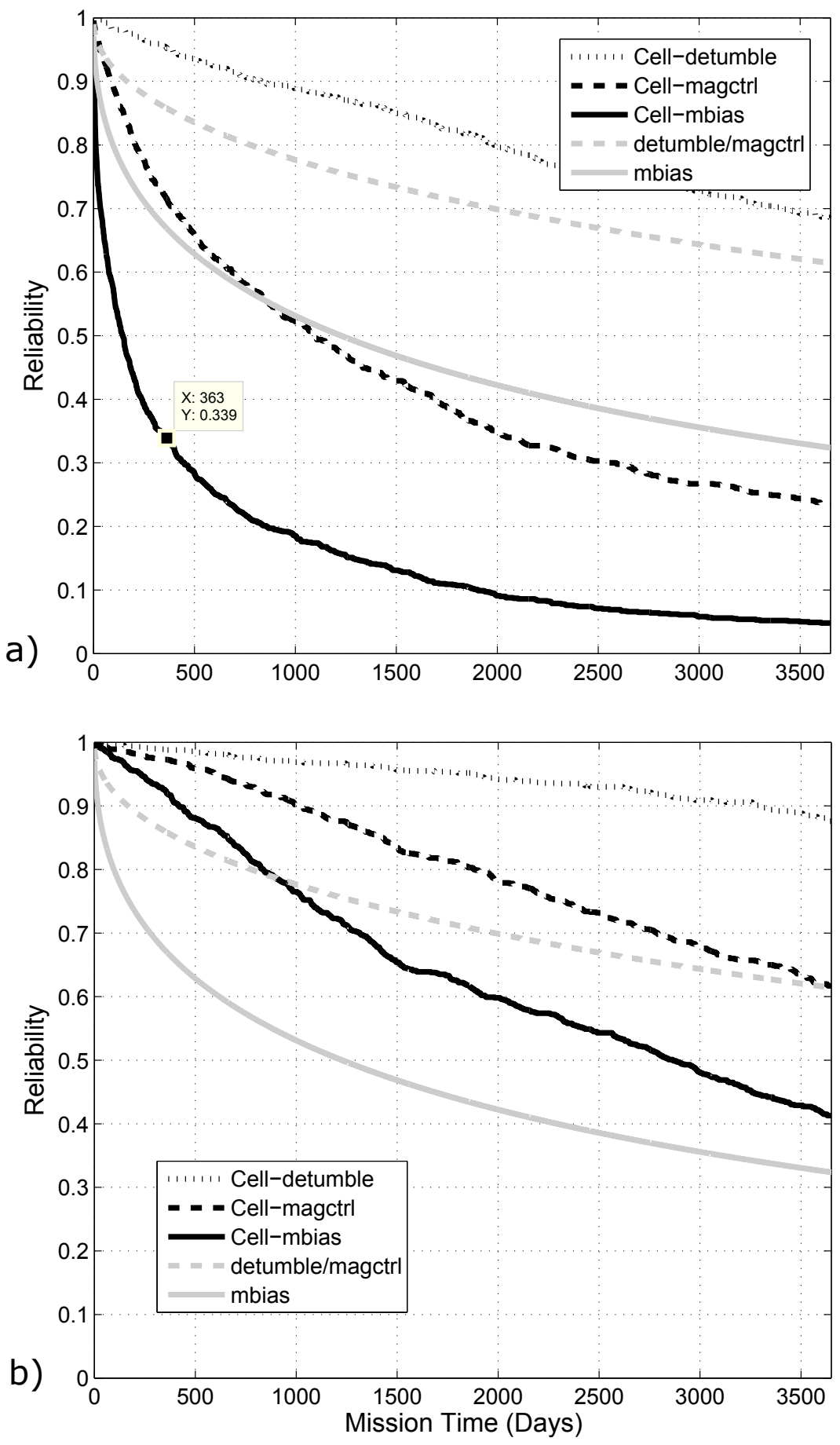

Figure 7.22: Comparison between the predicted reliabilities of the CubeADCS (operating in various modes) and the 2-cell (a) and 3-cell (b), theoretical, multicellular ADCSs. 
100 days. When operating in their most capable modes, the CubeADCS shows enhanced reliability.

For a real reliability advantage, an extra cell must be added to the cell-based design. Since the task allocation strategy is distributed, adding another cell is simple. All it involves is linking up the inter-cellular bus and further cross-strapping desired peripherals to the new cell. The three-cell ADCS is predicted to be more reliable than the CubeADCS in all modes, throughout its mission lifetime, as shown in Figure 7.22b. However, the three-cell ADCS comes with higher volume, mass and power consumption overheads, as will be described in Section 7.3.1.2.

The poor performance of the two-cell ADCS in this design is due to the small total number of proteins, and large number of peripherals, both of which restrict reconfigurability. The peripheral ratio of the system is $1 / 2(0.5)$, which results in far poorer reliability than a pure computational or perfectly cross-strapped system. The analysis of Chapter 6 highlighted the fact that the reliability benefits of a multicellular system increase as the total number of proteins increase, even if the loading remains equal. Thus, a larger system requiring more proteins is required in order for a multicellular architecture to show its reliability advantage over more traditional architectures.

\subsubsection{Performance and Overheads}

As described before, each testbench cell has a processing capacity of 120.96 DMIPS and consumes approximately $390 \mathrm{~mW}$ when operating at maximum capacity. Therefore, the theoretical cell-based ADCS, composed of two cells, offers 242 DMIPS of processing capacity at $780 \mathrm{~mW}$ of power consumption. In comparison, the CubeADCS has a processing capacity of only 60.44 DMIPS, but consumes only $350 \mathrm{~mW}$. Its processing capacity is derived from the CubeComputer's ARM Cortex M3 MCU, clocked at $48 \mathrm{MHz}$ (1.25 DMIPS/MHz [113]) and the two CubeControl, 8-bit, PIC18F MCUs, clocked at $11 \mathrm{MHz}$ each $(0.02$ DMIPS/MHz [114]). Thus, the cell-based ADCS offers four times more performance at a cost of twice the power consumption.

When placed in 'power-saving' mode by clocking the proteins down to $12 \mathrm{MHz}$, the cellbased ADCS offers almost exactly the same performance as the CubeADCS: 60 DMIPS. In this mode, the cell-based ADCS's power consumption is reduced to $620 \mathrm{~mW}$, but this is still $77 \%$ more than the CubeADCS. 
Table 7.4: Summary and comparison of CubeADCS and cell-based ADCS characteristics

\begin{tabular}{|c|c|c|c|}
\hline & CubeADCS & $2 \times 12 \mathrm{MHz}$ Cells & $3 \times 12$ MHz Cells \\
\hline $\begin{array}{l}1 \text { Year Reliability (\%) } \\
\text { Detumble } \\
\text { Magnetic Control } \\
\text { Momentum Bias }\end{array}$ & $\begin{array}{l}85.8 \\
66.8 \\
66.8\end{array}$ & $\begin{array}{l}95.2(+11 \%) \\
71.4(+6.9 \%) \\
33.4(-50 \%)\end{array}$ & $\begin{array}{l}98.8(+15 \%) \\
97.2(+46 \%) \\
91.9(+38 \%)\end{array}$ \\
\hline Performance (DMIPS) & 60.44 & 60.48 & 90 \\
\hline $\begin{array}{l}\text { Power Consumption }(W) \\
\text { Detumble } \\
\text { Magnetic Control } \\
\text { Momentum Bias }\end{array}$ & $\begin{array}{l}0.26 \\
0.35 \\
0.35\end{array}$ & $\begin{array}{l}0.41(+58 \%) \\
0.55(+57 \%) \\
0.62(+77 \%)\end{array}$ & $\begin{array}{l}0.56(+115 \%) \\
0.70(+100 \%) \\
0.78(+123 \%)\end{array}$ \\
\hline PCB Area $\left(\mathrm{cm}^{2}\right)$ & 200 & 200 & 300 \\
\hline
\end{tabular}

In terms of volume, both the cell-based ADCS and CubeADCS are composed of two PCBs of approximately $10 \times 10$ centimetres each, plus the same accompanying peripherals. Therefore, the two-cell ADCS comes with no volume overheads, while the three-cell ADCS incurs a 50 $\%$ overhead. The small size of the testbed cells makes them CubeSat compatible. However, their large power consumption overheads, even in 'power-saving' mode, leads to the same conclusion as the reliability comparison: a physically larger system, with more room for solar panels and more use for increased processing capacity, is a better target for cellularisation. Such a system is investigated in the next case study.

A comparison summary is given in Table 7.4.

\subsubsection{MicroSat Avionics}

While some commercial entities, such as PlanetLabs, have based their business on the nanosatellite form factor, which falls towards the bottom end of the small, low-cost satellite scale, others such as SSTL, argue that slightly larger platforms represent the optimum compromise between cost and capability [115]. Microsatellites fall in the range of 10-100 $\mathrm{kg}$ and compared to nanosatellites, such as CubeSats, have additional volume, mass and power available. These extra resources can be used to implement a level of redundancy. For example, SSTL sells a range of microsatellite buses which typically include dual-string, cross-strapped avionics. One such satellite, AlSat1-B, is shown in Figure 7.23 This case study focusses on cellularising a set of theoretical, traditional microsatellite-scale avionics.

The system architecture of the traditional microsatellite's avionics is inspired by that of a typical SSTL microsatellite. However, because SSTL does not publicly provide MTTF data 
on their subsystems, MTTF values are assumed for comparison purposes. These MTTF values are not representative of real SSTL hardware.

Table 7.5 lists the representative subsystems which could feasibly be functionally replaced by the current generation testbed cells. Certain subsystems, such as the electrical power system and communication subsystems, currently contain too much custom analogue circuitry to be feasibly replaced. However, future generations of artificial cells, through the advancement of technologies such as software defined radio, are envisioned to be even more multifunctional.

The traditional microsatellite has its main subsystems duplicated in cold redundancy, as is common amongst SSTL satellites. The traditional microsatellite's OBC is given a representative processing capacity of approximately 500 DMIPS, requiring 10 testbed cells (in power saving mode) to match its performance [117]. The OBC's workload is assumed to be composed of several tasks, allowing a natural distribution of the tasks onto the discrete protein processors of the testbed cells. The other subsystems are primarily peripheral interfaces and are based on 8-bit MCUs. Therefore, replacing them with cells is simply a matter of ensuring that sufficient generic I/O channels are provided.

Similary to the CubeADCS, the microsatellite avionics suite is modelled as being able to operate in three modes of increasing capability. In the simplest mode, detumble, at least one OBC and one ADCS interface are required. The ADCS interface acts as an interface to magnetorquers, a magnetometer and a sun sensor. Both the OBC and ADCS interface are modelled in cross-strapped, cold redundancy. The next mode, three-axis control, requires the addition of at least three reaction wheels. Typically, four wheels are included, giving some redundancy. The four wheels are modelled as a k-out-of-n system, requiring at least three to be active. Finally, full operational mode requires the addition of a propulsion interface and gyroscope interface, both of which are modelled in cross-strapped cold redundancy.

To determine the number of cells which will make up the cell-based system, the requirements of each operational mode must be determined. In detumble mode, the $\mathrm{OBC}$ is assumed to be operating at $50 \%$ capacity, requiring at least five proteins, with a further two proteins required for the magnetorquer and magnetometer interfaces. In three-axis mode, at least 14 proteins are required, with eight going towards the $\mathrm{OBC}$ operating at $80 \%$ capacity and the remaining six devoted to peripheral interfaces. Finally, full operational mode requires the $\mathrm{OBC}$ to be operating at $100 \%$ capacity, requiring 10 proteins, and a further eight are required for peripheral interfacing. Based on these numbers, and the desire to match the traditional system's level of redundancy, a multicellular system of 10 testbed cells (40 proteins total - 30 available to the user) is considered. 


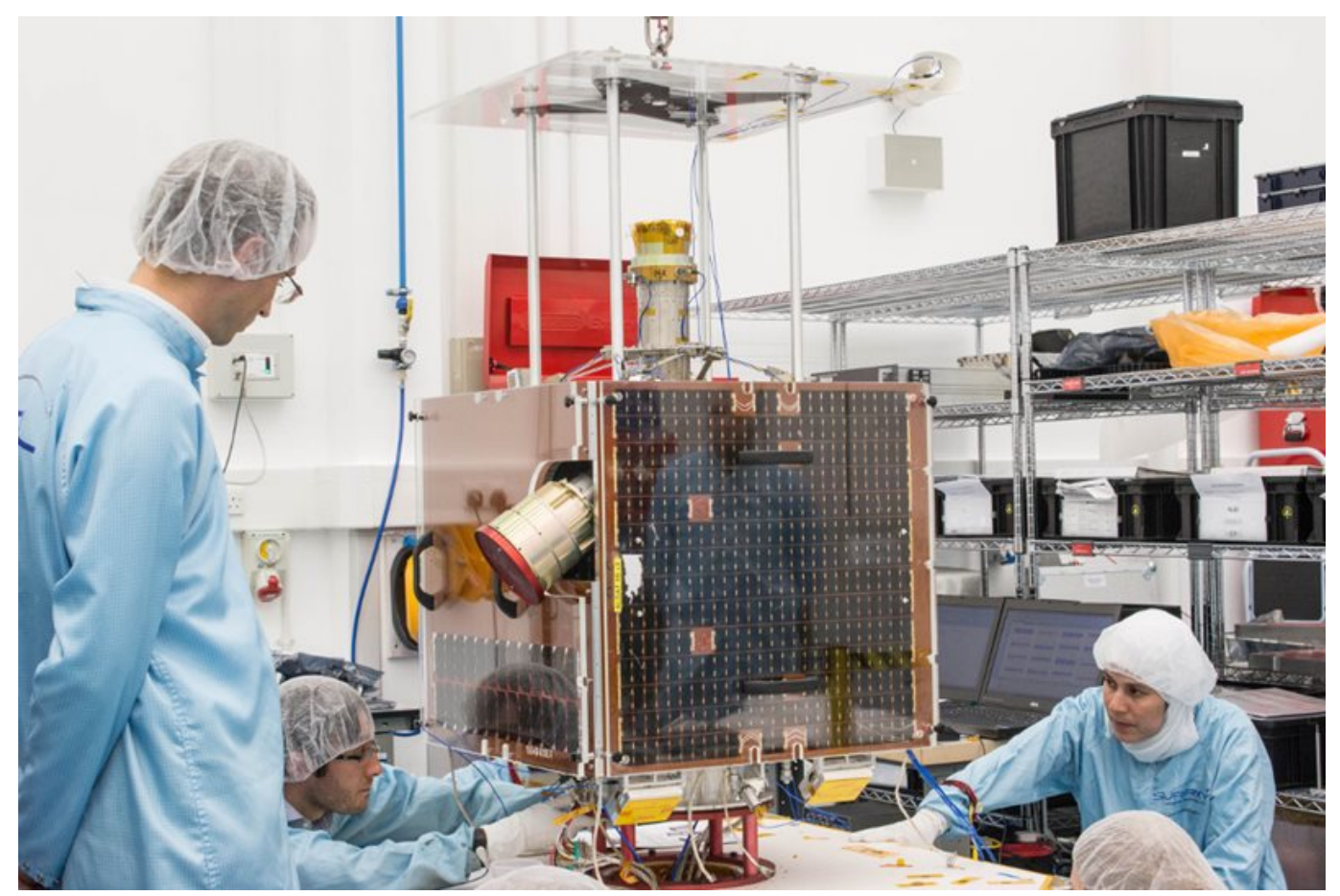

Figure 7.23: SSTL's 100kg AlSat-1B microsatellite during the assembly phase [116].

Table 7.5: Representative microsatellite subsystems, their processing capacity, associated peripherals, and equivalent number of proteins

\begin{tabular}{|l|l|l|l|l|}
\hline Subsystem & Redundant & DMIPS & Peripherals & $\begin{array}{l}\text { Eq. Proteins } \\
\text { (per sub.) }\end{array}$ \\
\hline OBC & cold & 500 & none & 10 \\
\hline ADCS Interface & cold & $<10$ & $\begin{array}{l}3 \times \text { magnetorquers } \\
1 \times \text { magnetometer } \\
1 \times \text { fine sun sensor }\end{array}$ & 3 \\
\hline Reaction Wheel & 3-out-of-4 & $<10$ & $\begin{array}{l}1 \times \text { BLDC Motor } \\
1 \times \text { heater } \\
2 \times \text { valves }\end{array}$ & 1 \\
\hline Propulsion Subsystem & cold & $<10$ & $1 \times$ digital gryoscope & 1 \\
\hline Gyroscope Interface & cold & $<10$ & & \\
\hline
\end{tabular}


Due to the large number of proteins required to match the performance of the $\mathrm{OBC}$, a large number of generic I/O channels are available for cross-strapping. Thus, the detumble peripherals (magnetometer and magnetorquers) are interfaced to six proteins each. The additional peripherals required for three-axis mode (sun sensor and three reaction wheels) are interfaced to five proteins each. Finally, the gyroscope and propulsion systems are interfaced to four proteins each. This gives a total of 40 interfaces, meaning every protein in the system is interfaced to a peripheral. Failure of the peripherals themselves, for example wear-out of a bearing in a reaction wheel motor, is not modelled. However, to guard against such failures, two or three of the same peripherals could be included in the system, splitting the available interfaces.

\subsubsection{Reliability Comparison}

As in the CubeSat case study, independent Weibull failure distributions are assumed, with a shape parameter of 0.4464 . In this case, the scale parameters of the subsystems, common and supporting components $\left(48 e^{3}, 35 e^{8}, 35 e^{7}\right)$ were adjusted to give the avionics an expected lifetime (MTTF) of five years. Figure 7.24 shows a screenshot of the MATLAB-based multicellular system simulator, configured for this case study. The resultant reliability plots, derived through analytical means for the traditional microsatellite's avionics and simulation for the multicellular system, are shown in Figure 7.25. In contrast to the CubeSat-scale system, the microsatellite-scale multicellular architecture shows a reliability advantage over the traditional, cross-strapped design, in all modes of operation. Three years into the simulated lifetime, the cell-based design is predicted to be $5 \%$ more reliable in detumble mode, and $18 \%$ more reliable in three-axis and full operational modes. Alternatively, this result implies that some cells could be removed from the multicellular system, lowering overheads, while matching the traditional microsatellite's avionics' reliability.

\subsubsection{Performance and Overheads}

In terms of power consumption, the cell-based design consumes less than $4 \mathrm{~W}$, while a traditional microsatellite OBC, such as the SSTL OBC750, itself consumes 10W [117]. This power saving is largely due to the use of modern, low power MCUs in the cell-based design. Furthermore, the cell-based design has the ability to linearly scale its power consumption with processing capacity by physically shutting off proteins. 


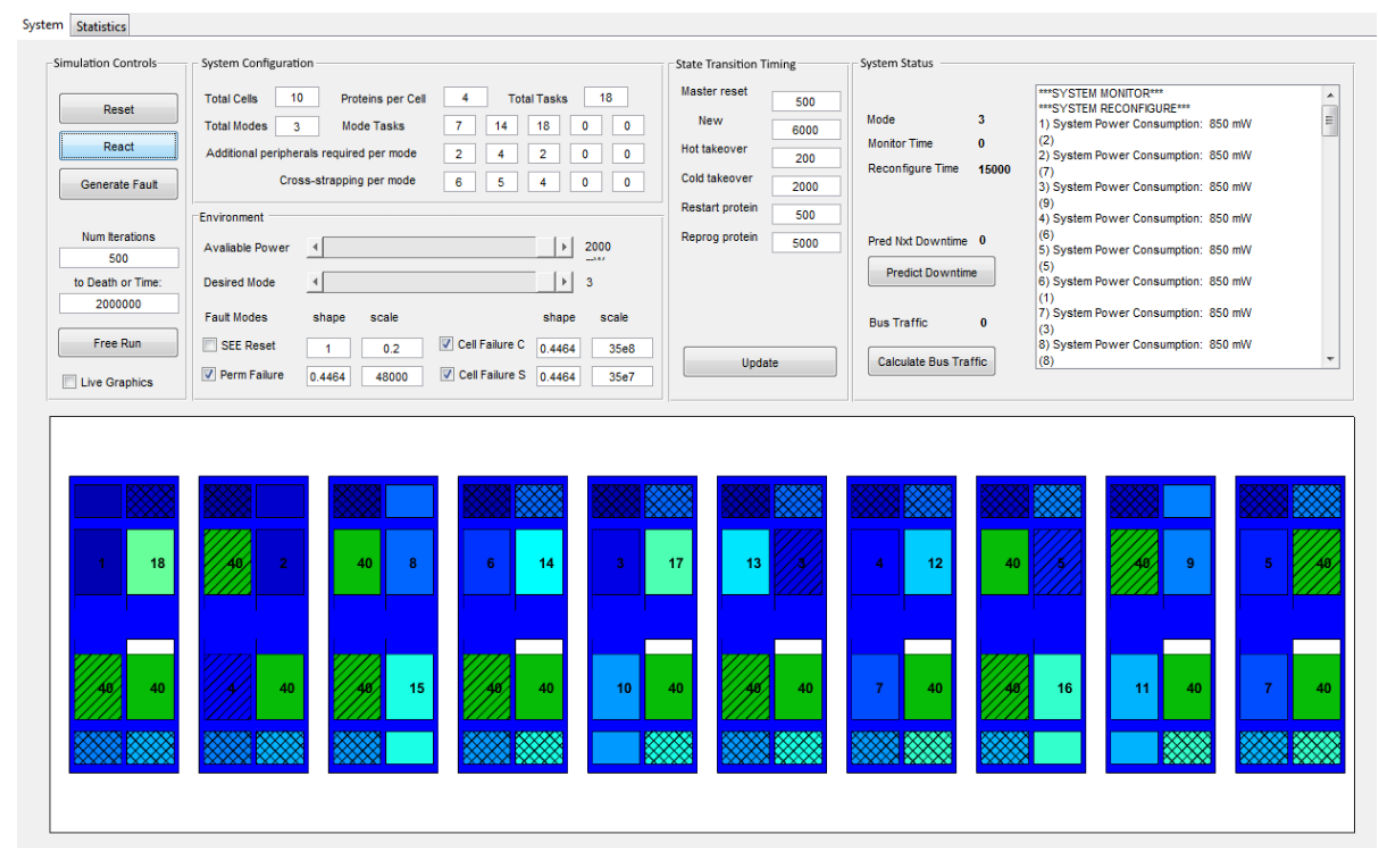

Figure 7.24: A screenshot of the MATLAB-based multicellular system simulator, configured to simulate a 10 -cell system.

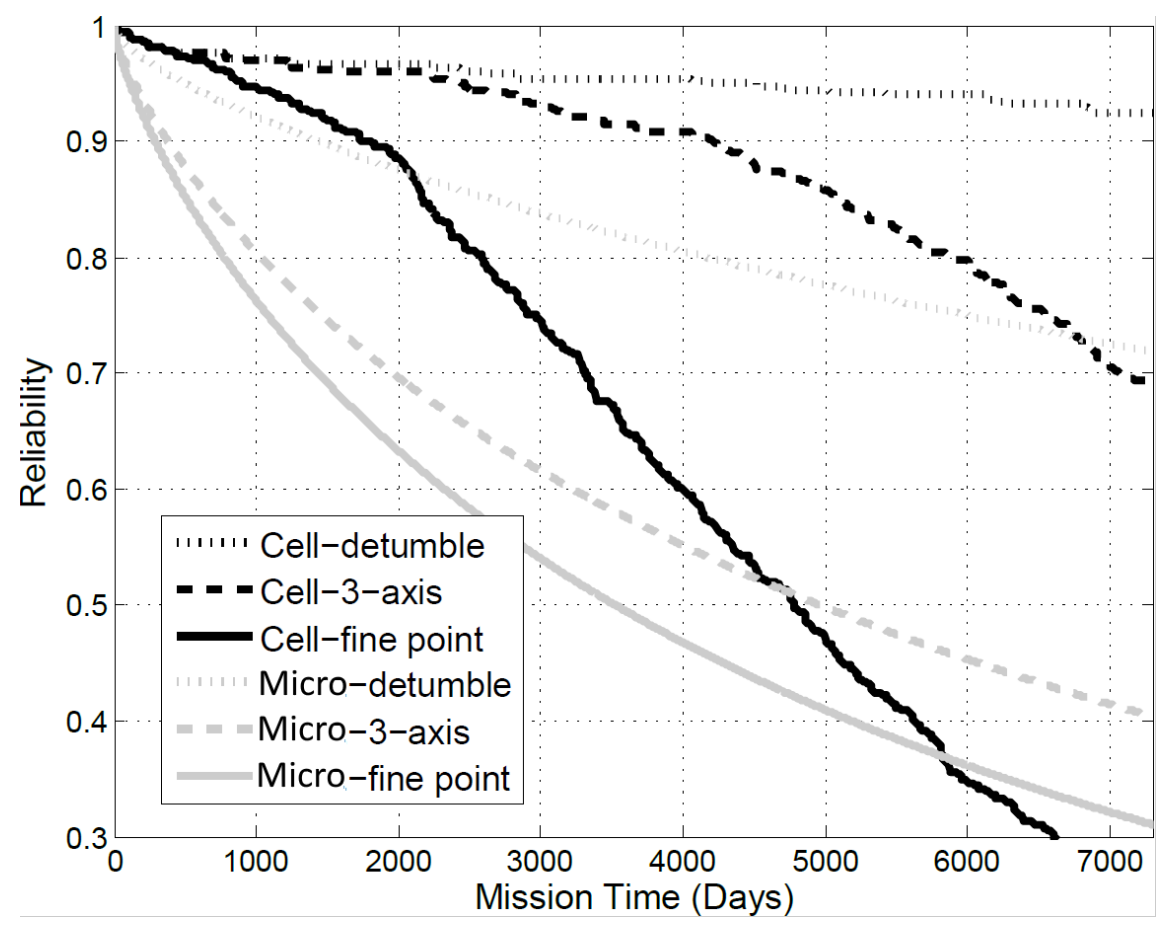

Figure 7.25: Comparison between the predicted reliability of a traditional cross-strapped microsatellite avionics suite, and a comparable 10-cell multicellular system. 
To compare volumes, the subsystems of the traditional microsatellite are said to occupy roughly $300 \times 150 \times 30 \mathrm{~mm}$ each, which is a standard format in which most SSTL subsystems are available [117]. At least three testbed cells, in their current format, can fit into this volume. Thus, while the theoretical microsatellite requires eight of these volumes (excluding the reaction wheels which have integrated electronics), the cell-based system requires only four. While this seems like a large volume saving, it is important to note that the SSTL avionics suite looses a lot of volume due to the predefined module boxes. Therefore, the most important outcome of the volume comparison is the fact that the cell-based solution can easily fit within the existing avionics volume.

\subsubsection{Case Studies Conclusion}

The microsatellite avionics could be successfully cellularised because the OBCs translate into a large number of purely-computational proteins. These proteins have the advantage of easily trading tasks between themselves, and offer a lot of peripheral cross-strapping possibilities. In all modes of operation, the cell-based design requires a larger number of functional 'units' than the traditional, cross-strapped design. In the traditional microsatellite avionics, each of the items in Table 7.5 represent one unit, while each protein represents a unit in the cell-based design. Since each unit was modelled with the same reliability, intuition would suggest that the system requiring more units should be less reliable. However, once reliability is introduced (e.g. at least one of the two OBCs must be functional is equivalent to at least 10 out 20 proteins must be functional), the benefits of the k-out-of-n architecture begin to outweigh the compounded unreliability of individual units. Thus, given there is some room for redundancy, as is the case in the microsatellite example, splitting individual, traditional subsystems into a number of proteins is beneficial.

In comparison to the CubeSat-scale system, the microsatellite system further benefits form a much lower peripheral ratio. While the multicellular CubeADCS had a peripheral ration of $1 / 2$ (meaning one peripheral for every two proteins), the microsatellite-scale system has a peripheral ratio of only 1/5. As was shown in Section 6.5 of Chapter 6, a peripheral ratio of $1 / 5$ is sufficiently low that the system behaves almost like a purely-computational, or perfectly cross-strapped, system. 


\subsection{Summary}

This chapter described two successful implementations of systems based on multicellular concepts. The first implementation, which forms the payload interface computer of the Surrey Space Centre's SMESAT CubeSat, is based on a single cell of four proteins. While it did not feauture generic I/O circuitry or the HARP middleware, it nonetheless allowed a complex subsystem to be developed by a single engineer, working part-time, in under a year. In addition, it gives SMESAT graceful degradation in experimental output by removing potential single points of failure.

The second implementation, based on two artificial cells of four proteins each, forms a complete Satellite Stem Cell architecture testbed. The testbed cells are approximately 10 $\mathrm{x} 10 \mathrm{~cm}$ each, contain six generic I/O channels per protein, and use ARM Cortex M0 microcontrollers as their protein processors. CAN buses, operating at $100 \mathrm{~kb} / \mathrm{s}$, form the inter-protein and inter-cellular communications links. Operating at maximum capacity, each cell offers 120.96 DMIPS of user processing capacity, and consumes approximately $390 \mathrm{~mW}$. Power savings can be made by clocking the protein processors at lower frequencies.

The HARP middleware, implemented for the testbed system, executes on top of the free, real-time operating system, FreeRTOS. The middleware consists of two parts: a set of agency services which execute on the macromolecular machinery protein, and an agent template. The services firmware has a memory footprint of just $28.7 \mathrm{kB}$ and requires less than $8 \mathrm{kB}$ of RAM, largely due to the offloading of the agent execution environment to dedicated processors. The agent template, which includes functions for ACL communication and generic I/O control, occupies just $24.9 \mathrm{kB}$ of memory.

A series of experiments were successfully conducted on the testbed to exercise the full functionality of the artificial cell hardware and HARP middleware. Additionally, through a set of benchmarking experiments, the scaling potential of the system was investigated. These experiments highlighted the large overheads associated with ACL communication, which limits practical health-check rates to $<1 \mathrm{~Hz}$, and AMS transactions to 20 per second. Nevertheless, with a $0.1 \mathrm{~Hz}$ health-check rate, the testbed system was able to recover from partial hardware failures in under 30 seconds. While not suited for systems requiring failoperational capability, a 30 second recovery time is acceptable for satellites in orbit, especially if it negates the need for ground operator intervention.

In the second part of the chapter, two case studies were presented. These focussed on the theoretical reliability benefits and practical overheads of 'cellularising' sets of traditional 
satellite avionics with the testbed cells. The case studies showed that the Satellite Stem Cell architecture makes better use of available redundancy capacity than traditional architectures. Cellularised microsatellite avionics are predicted to be up to $18 \%$ more reliable after three years, while consuming less power and occupying a similar volume. However, the case studies also showed that the Satellite Stem Cell architecture is poorly suited to very small systems, such as CubeSats, which have minimal room for redundancy and very limited power. 


\section{Chapter 8}

\section{Conclusions}

Despite their humble beginnings as educational projects for students and hobbyists, small, low-cost satellites have graduated on to challenging the roles of large, traditional satellites, while standing poised to enable exciting new mission concepts. However, the cost cutting measures which have been experimental in their success, have also led to poor on-orbit reliability to date. While traditional techniques for improving reliability are well known to satellite developers, their implementation is hampered by the intrinsic mass, volume, power, and budgetary limitations of small, low-cost satellites. Thus, this work sought to develop an alternative system architecture, which minimises overheads while maximising reliability.

To initially gain a better understanding of the causes of unreliability amongst small, low-cost satellites, a parametric reliability survey of 159 CubeSats was undertaken. The on-orbit performance of each CubeSat was compared against parameters of the CubeSat design, including number of redundant subsystems, number of COTS subsystems, ADCS complexity, and development time. Certain trends, such as increased redundancy leading to better reliability, were expected. However, a number of unexpected trends were discovered, too. These include an inverse relationship between development time and reliability, and a lower than expected optimal number of COTS subsystems. The study concluded that there was no clear recipe-for-success, as a large number of additional factors, which were not included in the survey, impact reliability, too. These include team experience and continuity, mission scope, available facilities, and budget. Nevertheless, the recently launched QB50 project will present a good opportunity to redo this study, as the 50 CubeSats it entails were all developed by different university teams, but with similar mission scopes and under similar time lines. 
With no clear solution provided by the CubeSat survey, Chapter 3 investigated the techniques used by biological life to thrive in even the harshest environments on Earth. Unicellular life was found to employ techniques which are very similar to those used on traditional satellites. These include the use of specially adapted proteins (radiation-hardened parts), genetic redundancy (functional redundancy), and gene repair (error detection and correction schemes). Multicellular life, on the other hand, with its distributed nature, displays the use of techniques which are not commonly employed by man-made systems. Through the biological processes of differentiation and inter-cellular communication, biological cells can start out identically, before taking on specialisations. Furthermore, these specialisations can change in response to damage or changing environmental conditions. Based on these multicellular concepts, an outline for a conceptual system architecture, based on initially identical, reconfigurable hardware blocks, instead of unique, discrete subsystems, was proposed.

Similar concepts can be found in several other research fields, including bio-inspired processors, agent computing, and modular spacecraft concepts. Important works from each of these fields were discussed, and the key novelties of the proposed multicellular architecture were highlighted.

Expanding on the conceptual system architecture proposed in Chapter 3, Chapter 4 presented the design of an artificial cell, which is expected to serve as the building block of multicellular systems. The differentiation process of a biological cell represents a unique solution to the complex problem of getting a single block of hardware to perform a wide variety of functions. Therefore, the artificial cell is loosely modelled on the architecture of a biological cell. In place of proteins and macromolecular machinery, the artificial cell has discrete processing elements, such as microcontrollers, and in place of a cell membrane, it has a set of novel, generic I/O circuitry. In a process which mimics differentiation, the processing element performing the role of macromolecular machinery can respond to changing internal and external conditions by programming different sets of firmware, stored in its non-volatile 'DNA' memory, into the protein processors. As both the macromolecular machinery and proteins are implemented using the same processing elements (based on the fact that biological macromolecular machinery is, itself, composed largely of proteins), the macromolecular machinery can be moved around the cell, preventing it from becoming a single point of failure.

To facilitate communication and cooperation amongst these artificial cells, a set of middlware was developed and described in Chapter 5. The middleware, name the Hybrid Agent Real-Time Platform, is based on the Foundation for Intelligent Physical Agents Abstract 
Architecture. It turns every artificial cell into an agency by executing a set of services on the macromolecular machinery processor. Due to the need for a small memory footprint and real-time capabilities, the HARP middleware distinguishes itself from other agent platforms in three areas. Firstly, the HARP middleware uses discrete processors, instead of virtual machines or interpreters, as its agent execution environment. This has the advantage of reducing the agency memory footprint and enabling agents to perform real-time tasks. Secondly, the HARP communication stack makes use of ISO-TP over CAN 2.0A as its transfer level protocol, cutting out resource-intensive layers such as HTTP and IIOP. In addition, the communication stack allows real-time CAN traffic to share the network with agent communication language messages. Finally, the HARP middleware embeds a peer-topeer task manager in each agency, allowing systems which are built using the artificial cells and HARP middleware to autonomously reconfigure in the event of failures.

The Satellite Stem Cell architecture, which entails the artificial cell hardware and HARP middlware, represents a compromise between fully centralised, and fully decentralised, architectures. It mimics the two-tiered architecture of simple multicellular organisms such as jellyfish, which have no central brains. Task allocation is coordinated in a peer-to-peer fashion at a cellular level, while task execution occurs at the protein level. Thus, it does not have a potential single point of failure, while also leaving its processing elements (proteins) unburdened from system-level decision making. What distinguishes the Satellite Stem Cell architecture from other multi-tiered architectures is the blurry line between the tiers. Since the macromolecular machinery, which represents the top tier, is composed of the same hardware as the proteins (bottom tier), the system can continuously reconfigure in the event of failures to ensure the two-tiered architecture remains functional. Furthermore, the Satellite Stem Cell architecture represents more than a computational system, as every protein has its own generic I/O hardware, allowing complex sensor/actuator systems to be developed without additional electronics.

In Chapter 6 the theoretical reliability of multicellular systems was investigated and compared to systems with traditional forms of reliability. First, a reliability equation for multicellular systems was derived, based on an extension of k-out-of-n system theory. Each cell is evaluated as an independent, multi-state, k-out-of-n system, before the full system reliability is calculated through the convolution of the individual cell results. Determining the optimal configuration of cells per system and proteins per cell proved to be complex, as different configurations were shown to be optimal at different points during a system's lifetime.

Nevertheless, trends were identified and compiled into a set of guidelines for multicellular system development. These include the advice that the benefits of multicellular architec- 
tures increase as the system complexity increases, and that heavy system loading favours configurations featuring fewer, larger cells. Overall, the analytical reliability equations predict promising improvements over traditional forms of reliability, provided the system is configured correctly.

To verify the derived analytical reliability equations, a MATLAB-based, multicellular system simulator was developed. The simulator features a graphical user interface and allows Monte Carlo simulations to be run. Using the same simulator, the effect of peripherals on the reliability of multicellular systems was investigated. The ability to interface directly to a variety of peripherals is one of the most important features of the Satellite Stem Cell architecture. However, adding peripherals to a system causes a negative deviation from the analytically predicted reliability, as the peripheral interfaces limit reconfigurability. However, it was shown that the derived reliability equations can still be used to reasonably predict the reliability of a multicellular system with peripherals, provided the peripheral ratio stayed below $1 / 6$.

To complement the established theoretical reliability benefits of multicellular architectures, Chapter 7 investigated their practical feasibility. Two systems based on multicellular concepts were successfully implemented. The first implementation forms the payload interface computer of the SMESAT CubeSat. While it does not feature generic I/O circuitry or the HARP middleware, it nonetheless allowed a complex subsystem to be developed by a single engineer, working part-time, in under a year. Additionally, it allows SMESAT to sustain partial hardware failures without losing all experimental output.

The second implementation forms a complete Satellite Stem Cell architecture testbed. Based on four ARM Cortex M0 MCUs, each 10 x $10 \mathrm{~cm}$ testbed cell offers 120 DMIPS of user processing capacity and consumes approximately $390 \mathrm{~mW}$. An implementation of the HARP middleware, which executes on top of the free, real-time operating system FreeRTOS, was developed for the testbed hardware. With a compiled size of only $28.7 \mathrm{kB}$, the HARP middleware is one of the smallest agent platforms.

A set of experiments were conducted to demonstrate the full functionality of the Satellite Stem Cell architecture, and to measure its overheads and scalability. Due to the large overheads incurred by ACL communication, the current hardware limits practical health-check rates to $<1 \mathrm{~Hz}$ and maximum AMS requests to 20 per second. Nevertheless, the testbed system demonstrated recovery from partial hardware failures within 30 seconds, which is acceptable for most earth-orbiting satellites. 
Chapter 7 concluded with two case studies which were based on the concept of cellularising sets of traditional satellite avionics using the testbed cells. While CubeSat-scale systems proved to be a poor fit for cellularisation, due to insufficient complexity and available power, cellularised microsatellite-scale systems were shown to benefit from enhanced reliability and reduced overheads.

Thus, despite the limitations of the current artificial cell hardware, the Satellite Stem Cell architecture was shown to form a practical and feasible alternative to current low-cost satellite architectures. The initial development of the proposed architecture is more complex than that of a system based on unique, discrete subsystems. However, once the artificial cell hardware is in production and the middleware has been fully tested, the development of future systems becomes streamlined. This process should simply involve determining the required number of cells, based on processing capacity, I/O, and redundancy requirements, and developing a set of template-based agents to perform system tasks.

\subsection{Publications}

The work described in this thesis has been (or is expected to be) published in the following journals and fully peer-reviewed conference proceedings:

- A. O. Erlank and C. P. Bridges, 'A Hybrid Real-Time Agent Platform for Fault-Tolerant, Embedded Applications' revision submitted for manuscript acceptance to the Journal of Autonomous and Multi Agent Systems [revision under review].

- A. O. Erlank and C. P. Bridges, 'Reliability Analysis of Multicellular System Architectures for Low-Cost Satellites' submitted for manuscript acceptance to Acta Astronautica [revision under review].

- A. O. Erlank and C. P. Bridges, 'The satellite stem cell architecture,' 2016 IEEE Symposium Series on Computational Intelligence (SSCI), Athens, 2016, pp. 1-8.

- A. O. Erlank and C. P. Bridges, 'A multicellular architecture towards low-cost satellite reliability,' 2015 NASA/ESA Conference on Adaptive Hardware and Systems (AHS), Montreal, QC, 2015, pp. 1-8.

- A. O. Erlank and C. P. Bridges, 'Satellite stem cells: The benefits \& overheads of reliable, multicellular architectures,' 2017 IEEE Aerospace Conference, Big Sky, MT, 2017, pp. 1-12. 
In addition, work related to the cellularised payload interface computer on SMESAT was presented at the 8th European CubeSat Symposium in 2016.

\subsection{Future Work}

Areas for future work include predicting the downtime of multicellular systems, investigating alternative task allocation strategies, implementing a semantic language, developing a complementary agent-based ground station, and building the next generation of artificial cells.

\subsubsection{Downtime Prediction}

In Chapter 6, analytical reliability equations for predicting the lifetimes of multicellular systems were derived. This work could be extended by developing techniques for predicting system downtime, or recovery time. Given the current system configuration, failure rate models, and the probabilistic times required to complete each phase of the recovery process (e.g. Fault detection, based on health check rate, and reprogramming time, based on program size), an absorbing Markov chain could be derived to model the recovery process. Using this model, the probabilistic time required to recover from the next failure could be calculated.

\subsubsection{Specialised Task Allocation Strategies}

The currently implemented, greedy task allocation strategy, explained in Section 5.2.2, is not globally optimum, but was chosen for its simplicity and robustness. Several alternative task allocation strategies, optimised for different situations, could be investigated and implemented. Potential aims of these strategies include:

1. Minimising inter-cellular bus traffic by grouping related tasks on common cells.

2. Maximising reliability by monitoring protein processor upset rates over time and matching task priority to processor reliability.

3. Load and priority balancing across cells to minimise recovery time after the failure of individual cells. 
Development and evaluation of alternative task allocation strategies can be done in the MATLAB-based, multicellular system simulator, described in Section 6.4.

\subsubsection{Semantic Language}

As described in Chapter 5, the HARP middleware does not currently implement a semantic language. The addition of a semantic language is required to unlock the full potential of agent computing. A semantic language gives structure to the ACL message contents, allowing diverse, intelligent agents to have complex interactions. Through a semantic language, agents can express beliefs and desires, and cooperate to find solutions to high-level problems. Welldocumented semantic languages include Knowledge Query Manipulation Language (KQML) and FIPA Semantic Language (SL). Both languages already have C implementations freely available. Thus, to extend the FIPA middleware with a semantic language would simply involve feeding the ACL message contents into the semantic language interpreter.

\subsubsection{Agent-Based Ground Station and Communication Gateways}

The utility of on-orbit agents can be enhanced by having them interact with an agent-based ground station. In this way, many of the operational and planning tasks normally performed by ground station personnel could be automated. For example, ground-station agents could find weather predictions online and communicate this information to on-orbit agents for planning payload operations. Similarly, ground station agents could monitor online sources for information regarding solar weather and warn on-orbit agents to reconfigure the satellite into a safe mode in anticipation of solar flares. An agent-based ground station can be based on a traditional, FIPA-compatible agent platform, such as JADE, and communicate with HARP agents via a gateway. Since the HARP protocol stack is FIPA compatible from the bitwise-encoding layer upwards, a gateway would be simple to implement. The gateway could be in the satellite's transceiver, or, more likely, in the ground station.

\subsubsection{Next Generation Artificial Cells}

The current-generation artificial cells have proven the practical feasibility of a reconfigurable hardware building block. However, several areas of the design have room for improvement. The generic I/O circuitry output MOSFETs must be replaced as they have too much parasitic capacitance, preventing high-speed digital peripherals from being reliably cross-strapped. In 
addition, analogue and digital switches could be included to configure each channel, which would remove the current need for minor component modifications between operating modes. To implement a semantic language and other middleware improvements, the flash memory of the protein processors must be increased, as the current version of the HARP middleware occupies $90 \%$ of the available memory. Increases in the CAN bus speed would be beneficial, too, as ACL traffic is expected to rise as agents become more intelligent.

Finally, it would be interesting to investigate the theoretical reliability and practical feasibility of a cell implemented mostly in a single FPGA or ASIC. Such a design may show improved reliability as components and solder joints are minimised. However, in a traditional FPGA, this advantage may be outweighed by the vulnerability of the common and support circuitry around a set of softcore protein processors. Furthermore, while a small number of mixedsignal FPGAs exist, such as the original Actel SmartFusion, the included analogue circuitry is not capable of dealing with high-power signals. An ASIC solution, however, has the same advantages of reduced components and solder joints, while allowing the inclusion of power MOSFETs and carefully designed common and support circuitry. 


\section{References}

[1] M. Swartwout, "The first one hundred CubeSats : A statistical look," Journal of Small Satellites, vol. 2, no. 2, pp. 213-233, 2013.

[2] W. Harwood, "NASA launches $\$ 855$ million Landsat mission," 2013. [Online]. Available: http://www.cbsnews.com/news/nasa-launches-855-million-landsat-mission/

[3] P. B. de Selding, "First of Europe's Sentinel Earth Observing Satellites Reaches Orbit," 2014. [Online]. Available: http://spacenews.com/ 40104first-of-europes-sentinel-earth-observing-satellites-reaches-orbit/

[4] USGS, “Landsat Picture Gallery," 2012. [Online]. Available: https://landsat.usgs.gov/ landsat-picture-gallery

[5] "Final solar wing deployment test for Sentinel-2A," 2015. [Online]. Available: https://phys.org/news/2015-04-solar-wing-deployment-sentinel-2a.html

[6] I. Nikolova, "Microsatellites Advantages. Profitability and Return," in SPACE, ECOLOGY, SAFETY, Varna, Bulgaria, 2005.

[7] "PSLV-C37 Successfully Launches 104 Satellites in a Single Flight," 2017. [Online]. Available: http://www.isro.gov.in/update/15-feb-2017/ pslv-c37-successfully-launches-104-satellites-single-flight

[8] M. N. Sweeting, "Space at surrey: Microsatellites \& minisatellites for affordable access to space," COSPAR Colloquia Series, vol. 10, no. C, pp. 82-98, 1999.

[9] Planet Labs, “Our Approach.” [Online]. Available: https://www.planet.com/company/ approach/

[10] J. Puig-Suari, C. Turner, and W. Ahlgren, "Development of the standard CubeSat deployer and a CubeSat class PicoSatellite," in 2001 IEEE Aerospace Conference Proceedings (Cat. No.01TH8542), vol. 1. IEEE, 2001, pp. 1/347-1/353.

[11] W. Marshall and C. Boshuizen, "Planet Labs' Remote Sensing Satellite System,” 2013.

[12] C. Kilic, T. Scholz, and C. Asma, "Deployment strategy study of QB50 network of CubeSats," in RAST 2013 - Proceedings of 6th International Conference on Recent Advances in Space Technologies, 2013, pp. 935-939.

[13] M. N. Sweeting, "UoSAT-1: A review of orbital operations and results," Journal of the Institution of Electronic and Radio Engineers, vol. 57, no. 5, pp. S184-S194, 1987. 
[14] Surrey Satellite Technology Ltd, "CARBONITE-1: The mission," 2015. [Online]. Available: https://www.sstl.co.uk/Missions/CARBONITE-1-Launched-2015/ CARBONITE-1/CARBONITE-1-The-mission

[15] I. Klotz, "SpaceX seeks U.S. approval for internet-via-satellite network I Reuters," 2016. [Online]. Available: www.reuters.com/article/ us-space-spacex-idUSKBN13C085

[16] C. Underwood, S. Pellegrino, V. J. Lappas, C. P. Bridges, and J. Baker, "Using CubeSat/micro-satellite technology to demonstrate the Autonomous Assembly of a Reconfigurable Space Telescope (AAReST)," Acta Astronautica, vol. 114, pp. 112$122,2015$.

[17] J. L. Forshaw, G. S. Aglietti, N. Navarathinam, H. Kadhem, T. Salmon, A. Pisseloup, E. Joffre, T. Chabot, I. Retat, R. Axthelm, S. Barraclough, A. Ratcliffe, C. Bernal, F. Chaumette, A. Pollini, and W. H. Steyn, "RemoveDEBRIS: An in-orbit active debris removal demonstration mission," Acta Astronautica, vol. 127, pp. 448-463, 2016.

[18] B. Doncaster, C. Williams, and J. Shulman, "2017 Nano/Microsatellite Market Forecast," SpaceWorks Enterprises Inc., Atlanta, GA, Tech. Rep., 2017. [Online]. Available: http://spaceworksforecast.com/docs/ SpaceWorks $\left\{_{-}\right\}$Nano $\left\{_{-}\right\}$Microsatellite $\left\{_{-}\right\}$Market $\left\{_{-}\right\}$Forecast $\left\{_{-}\right\} 2017$.pdf

[19] H. Pham, System Software Reliability, 1st ed., ser. Springer Series in Reliability Engineering. London: Springer London, 2006.

[20] A. Avizienis, "Fault-Tolerant Systems," IEEE Transactions on Computers, vol. C-25, no. 12, pp. 1304-1312, 1976.

[21] J. H. Saleh and J.-F. Castet, Spacecraft Reliability and Multi-State Failures. Chichester, UK: John Wiley \& Sons, Ltd, mar 2011.

[22] B. Fleming, "Microcontroller Units in Automobiles [Automotive Electronics]," IEEE Vehicular Technology Magazine, vol. 6, no. 3, pp. 4-8, sep 2011.

[23] P. Bakkes and S. Mostert, "Fault tolerance in SUNSAT satellite," in 9th Computing in Aerospace Conference, 1993.

[24] J.-F. Castet and J. H. Saleh, "Satellite Reliability: Statistical Data Analysis and Modeling," Journal of Spacecraft and Rockets, vol. 46, no. 5, pp. 1065-1076, sep 2009.

[25] G. F. Dubos, J. F. Castet, and J. H. Saleh, "Statistical reliability analysis of satellites by mass category: Does spacecraft size matter?" Acta Astronautica, vol. 67, no. 5-6, pp. 584-595, 2010.

[26] J. Guo, L. Monas, and E. Gill, "Statistical analysis and modelling of small satellite reliability," Acta Astronautica, vol. 98, no. 1, pp. 97-110, may 2014.

[27] J. Bouwmeester and J. Guo, "Survey of worldwide pico- and nanosatellite missions, distributions and subsystem technology," Acta Astronautica, vol. 67, no. 7-8, pp. $854-862$, oct 2010. 
[28] M. Swartwout, "CubeSat Database." [Online]. Available: https://sites.google.com/a/ slu.edu/swartwout/home/cubesat-database

[29] A. R. Aslan, H. B. Yagci, M. E. Umit, A. Sofyali, M. E. Bas, M. S. Uludag, O. E. Ozen, M. D. Aksulu, E. Yakut, C. Oran, M. Suer, I. A. Akyol, A. B. Ecevit, M. S. Ersoz, I. Oz, S. Gulgonul, B. Dinc, and T. Dengiz, "Development of a LEO communication CubeSat," in RAST 2013 - Proceedings of 6th International Conference on Recent Advances in Space Technologies, 2013, pp. 637-641.

[30] E. Oland, "The HiNCube student satellite - Lessons learned," in 2015 7th International Conference on Recent Advances in Space Technologies (RAST). IEEE, jun 2015, pp. 429-432.

[31] S. Rossi, A. Ivanov, M. Richards, and V. Gass, "The SwissCube's technologies results after four years of flight," in International Astronautical Congress, 2013.

[32] J. Bouwmeester, M. Langer, and E. Gill, "Survey on the implementation and reliability of CubeSat electrical bus interfaces," CEAS Space Journal, sep 2016.

[33] S. Perez, N. Jarrix, N. J.-H. Roche, J. Boch, J.-R. Vaille, and A. Penarier, "ROBUSTA, a student satellite to serve the radiation effects community," in Small Satellite Conference, 2009.

[34] ESA, "CubeSats Satellite Operations Update," 2012. [Online]. Available: http://m.esa.int/Education/CubeSats $\left\{_{-}\right\}$satellite $\left\{_{-}\right\}$operations $\left\{_{-}\right\}$update

[35] R. Punnoose, A. Kemp, and C. Niederstrasser, "Mission Overview," 2014. [Online]. Available: https://www.tjhsst.edu/students/activities/tj3sat/

[36] California Polytechnic State University, "Missions Launched." [Online]. Available: http://www.polysat.org/launched/

[37] G. Cooper, "The Origin and Evolution of Cells," in The Cell: A Molecular Approach. 2nd edition. Sunderland (MA): Sinauer Associates, 2000.

[38] R. Harrison, B. Papp, C. Pál, S. G. Oliver, and D. Delneri, "Plasticity of genetic interactions in metabolic networks of yeast." Proceedings of the National Academy of Sciences of the United States of America, vol. 104, no. 7, pp. 2307-12, 2007.

[39] T. L. Hsiao and D. Vitkup, "Role of duplicate genes in robustness against deleterious human mutations," PLoS Genetics, vol. 4, no. 3, 2008.

[40] J. Zhang, "Genetic Redundancies and Their Evolutionary Maintenance," in Advances in Experimental Medicine and Biology, 2012, vol. 751, pp. 279-300.

[41] B. Alberts, A. Johnson, J. Lewis, D. Morgan, M. Raff, K. Roberts, and P. Walter, Molecular Biology of the Cell 6e, 2014, vol. 6, no. 6.

[42] J. D. Watson, T. a. Baker, S. P. Bell, A. Gann, M. Levine, and R. Losick, "Molecular biology of the gene," p. 768, 2003. 
[43] N. Geiling, “The Science Behind Yellowstone's Rainbow Hot Spring," 2016. [Online]. Available: http://www.smithsonianmag.com/travel/ science-behind-yellowstones-rainbow-hot-spring-180950483/

[44] A. Mihai, "Giant single celled organisms lurk on ocean's deepst point," 2011. [Online]. Available: http://www.zmescience.com/science/oceanography/ biggest-single-cell-xenophyophore-24102011/

[45] C. Gyles and P. Boerlin, "Horizontally transferred genetic elements and their role in pathogenesis of bacterial disease." Veterinary pathology, vol. 51, no. 2, pp. 328-40, 2014.

[46] P. De Selding, "SpaceX Opening Seattle Plant To Build 4,000 Broadband Sats," 2016. [Online]. Available: http://spacenews.com/ spacex-opening-seattle-plant-to-build-4000-broadband-satellites/

[47] D. J. Barnhart, T. Vladimirova, A. M. Baker, and M. N. Sweeting, "A low-cost femtosatellite to enable distributed space missions," Acta Astronautica, vol. 64, no. 11-12, pp. 1123-1143, 2009.

[48] D. J. Barnhart, T. Vladimirova, and M. N. Sweeting, "Very-Small-Satellite Design for Distributed Space Missions," Journal of Spacecraft and Rockets, vol. 44, no. 6, pp. 1294-1306, 2007. [Online]. Available: http://arc.aiaa.org/doi/10.2514/1.28678

[49] R. S. K. Barnes, P. P. Calow, P. J. W. Olive, D. W. Golding, and I. Spicer, "The Evolutionary History and Phylogeny of the Invertabrates," in The Invertabrates. John Wiley \& Sons, Ltd, 2001.

[50] A. Rokas, "The molecular origins of multicellular transitions," Current Opinion in Genetics \& Development, vol. 18, no. 6, pp. 472-478, dec 2008.

[51] M. K. Vickaryous and B. K. Hall, "Human cell type diversity, evolution, development, and classification with special reference to cells derived from the neural crest," Biological Reviews, vol. 81, no. 03, p. 425, aug 2006.

[52] E. Bianconi, A. Piovesan, F. Facchin, A. Beraudi, R. Casadei, F. Frabetti, L. Vitale, M. C. Pelleri, S. Tassani, F. Piva, S. Perez-Amodio, P. Strippoli, and S. Canaider, "An estimation of the number of cells in the human body," Ann Hum Biol, vol. 40, no. 6, pp. 301-4460, 2013.

[53] YourGenome, "Tiny fish, big splash: the story of the zebrafish." [Online]. Available: http://www.yourgenome.org/stories/tiny-fish-big-splash-the-story-of-the-zebrafish

[54] Z. Cormier, "Newt sequencing may set back efforts to regrow human limbs," 2013. [Online]. Available: http://www.nature.com/news/ newt-sequencing-may-set-back-efforts-to-regrow-human-limbs-1.12479

[55] C. Jopling, S. Boue, and J. C. Izpisua Belmonte, "Dedifferentiation, transdifferentiation and reprogramming: three routes to regeneration." Nature reviews. Molecular cell biology, vol. 12, no. 2, pp. 79-89, 2011. 
[56] E. Mostovenko, A. M. Deelder, and M. Palmblad, "Protein expression dynamics during Escherichia Coli glucose-lactose diauxie," BMC Microbiology, vol. 11, no. 1, p. 126, 2011.

[57] T. Matheson, "Invertebrate Nervous Systems," in Encyclopedia of Life Sciences. Chichester: John Wiley \& Sons, Ltd, oct 2002.

[58] A. J. Greensted, “A Reliability Engineered Multicellular Architecture Inspired by Endocrinology: The BioNode System," Doctor of Philosophy, The University of York, 2004.

[59] A. Cashin-Garbutt, "What is Epinephrine (Adrenaline)?" 2014. [Online]. Available: http://www.news-medical.net/health/ What-is-Epinephrine- $\{\%\} 28$ Adrenaline $\{\%\} 29$.aspx

[60] M. B. Miller and B. L. Bassler, "Quorum sensing in bacteria." Annual review of microbiology, vol. 55, pp. 165-99, 2001.

[61] S. Elmore, “Apoptosis: A Review of Programmed Cell Death,” pp. 495-516, 2007.

[62] D. Mange, E. Sanchez, A. Stauffer, G. Tempesti, P. Marchal, and C. Piguet, "Embryonics: a new methodology for designing field-programmable gate arrays with self-repair and self-replicating properties," IEEE Transactions on Very Large Scale Integration (VLSI) Systems, vol. 6, no. 3, pp. 387-399, sep 1998.

[63] C. Ortega and A. Tyrrell, "Reliability analysis of self-repairing bio-inspired cellular hardware," in Evolutionary Hardware Systems (Ref. No. 1999/033), IEE Half-day Colloquium on, 1999, pp. 2/1-2/5.

[64] D. Bradley, C. Ortega-Sanchez, and A. Tyrrell, "Embryonics+immunotronics: a bio-inspired approach to fault tolerance," in Proceedings. The Second NASA/DoD Workshop on Evolvable Hardware, vol. 44, no. 0. IEEE Comput. Soc, 2000, pp. 215-223.

[65] A. M. Tyrrell, E. Sanchez, D. Floreano, G. Tempesti, D. Mange, J. M. Moreno, J. Rosenberg, and A. Villa, "POEtic Tissue: An Integrated Architecture for Bioinspired Hardware," 5\{t\}h \{I\}nternational \{C\}onference on $\{E\}$ volvable $\{$ S $\}$ ystems (\{ICES\}'03), pp. 129-140, 2003.

[66] J. M. Moreno, Y. Thoma, E. Sanchez, O. Torres, and G. Tempesti, "Hardware realization of a bio-inspired POEtic tissue," in Proceedings - 2004 NASA/DoD Conference on Evolvable Hardware, 2004, pp. 237-244.

[67] M. R. Boesen and J. Madsen, "eDNA: A bio-inspired reconfigurable hardware cell architecture supporting self-organisation and self-healing," in Proceedings - 2009 NASA/ESA Conference on Adaptive Hardware and Systems, AHS 2009, 2009, pp. $147-154$.

[68] M. R. Boesen, D. Keymeulen, J. Madsen, T. Lu, and T. H. Chao, "Integration of the reconfigurable self-healing eDNA architecture in an embedded system," in IEEE Aerospace Conference Proceedings, 2011. 
[69] B. Chen, H. H. Cheng, and J. Palen, "Mobile-C: A mobile agent platform for mobile C/C++ agents," Software - Practice and Experience, vol. 36, no. 15, pp. 1711-1733, 2006.

[70] T. Finin, R. Fritzson, D. McKay, and R. McEntire, "KQML as an agent communication language," Proceedings of the third international conference on Information and knowledge management - CIKM '94, pp. 456-463, 1994. [Online]. Available: http://portal.acm.org/citation.cfm?doid=191246.191322\{\%\}5Cnhttp: //dl.acm.org/citation.cfm?id=191322\{\%\}5Cnhttp://portal.acm.org/citation.cfm? doid=191246.191322

[71] M. Henning, "The rise and fall of CORBA," Communications of the ACM, vol. 51, no. 8, p. 52, 2008. [Online]. Available: http://portal.acm.org/citation.cfm?doid= 1378704.1378718

[72] K. Kravari and N. Bassiliades, "A Survey of Agent Platforms," Journal of Artificial Societies and Social Simulation, vol. 18, no. 1, 2015.

[73] F. Bellifemine, G. Caire, and D. Greenwood, Developing Multi-Agent Systems with $J A D E$, ser. Wiley Series in Agent Technology. Chichester, UK: John Wiley \& Sons, Ltd, mar 2007.

[74] S. Poslad, P. Buckle, and R. Hadingham, "The FIPA-OS Agent Platform: Open Source for Open Standards," In Proceedings of the 5th International Conference and Exhibition on the Practical Application of Intelligent Agents and Multi-Agents, vol. 355 , p. 368, 2000.

[75] D. Krol and F. Nowakowski, "Practical performance aspects of using real-time multiagent platform in complex systems," in 2013 IEEE International Conference on Systems, Man, and Cybernetics, , 2013, pp. 1121-1126.

[76] F. Bergenti, A. Poggi, B. Burg, and G. Caire, "Deploying FIPA-compliant systems on handheld devices," IEEE Internet Computing, vol. 5, no. 4, pp. 20-25, 2001.

[77] M. Laukkanen, S. Tarkoma, and J. Leinonen, "FIPA-OS agent platform for smallfootprint devices," Intelligent Agents VIII, vol. 2333, pp. 447-460, 2002.

[78] J.-J. Meyer and F. Koch, "Knowledge-based Autonomous Agents for Pervasive Computing Using AgentLight," IEEE Distributed Systems Online, vol. 4, 2003.

[79] Oracle, "Frequently asked questions oracle java me embedded 8 and 8.1." [Online]. Available: http://www.oracle.com/technetwork/java/embedded/documentation/ me-e-otn-faq-1852008.pdf

[80] C.-L. Fok, G.-C. Roman, and C. Lu, "Agilla: A mobile agent middleware for selfadaptive wireless sensor networks," pp. 1-26, 2009.

[81] C. P. Bridges and T. Vladimirova, "Real-time agent middleware experiments on javabased processors towards distributed satellite systems," in IEEE Aerospace Conference Proceedings, 2011. 
[82] M. Zabel, T. B. Preuber, P. Reichel, and R. G. Spallek, "Secure, Real-Time and MultiThreaded General-Purpose Embedded Java Microarchitecture," in 10th Euromicro Conference on Digital System Design Architectures, Methods and Tools (DSD 2007). IEEE, aug 2007, pp. 59-62.

[83] D. Bernard, G. Dorais, E. Gamble, B. Kanefsky, J. Kurien, G. K. Man, W. Millar, N. Muscettola, P. Nayak, K. Rajan, N. Rouquette, B. Smith, W. Taylor, and Y.-W. Tung, "Spacecraft Autonomy Flight Experience : The DS1 Remote Agent Experiment," in Proceedings of the AIAA Space Technology Conference \& Exposition, 1999, pp. $1-11$.

[84] D. Surka, M. Brito, and C. Harvey, "The real-time ObjectAgent software architecture for distributed satellite systems," in 2001 IEEE Aerospace Conference Proceedings (Cat. No.01TH8542), vol. 6. IEEE, 2001, pp. 2731-2741.

[85] C. Ouch, "MATURITY ASSESSMENT OF SPACE PLUG-AND-PLAY ARCHITECTURE," MASTER OF SCIENCE IN SYSTEMS ENGINEERING MANAGEMENT, Naval Postgraduate School, 2013.

[86] J. Lyke, D. Fronterhouse, S. Cannon, D. Lanza, and W. T. Byers, "Space Plug-and-Play Avionics 3rd Responsive Space Conference," in 3rd Responsive Space Conference, 2005, pp. 1-12.

[87] F. Bruhn, "TechEdSat - CubeSat Technology demonstration mission featuring Plugand-play and radiation hardened electronics," in 9th Annual Spring CubeSat Developer's Workshop, San Luis Obispo, CA, 2012.

[88] D. Barnhart, L. Hill, M. Turnbull, P. Will, H. S. Telescope, A. Engineering, and S. E. Departments, "Changing satellite morphology through cellularization," in AIAA SPACE Conference and Exposition 2012, no. September, 2012, pp. 1-9.

[89] A. A. Kerzhner, M. O. Khan, M. D. Ingham, J. Ramirez, J. Hollman, J. de Luis, S. Arestie, and D. Sternberg, "Architecting Cellularized Space Systems using ModelBased Design Exploration," AIAA SPACE 2013 Conference and Exposition, pp. 1-24, 2013.

[90] D. Barnhart, L. Hill, E. Fowler, R. Hunter, L. Hoag, B. Sullivan, and P. Will, “A Further Look at Potential Impact of Satlets on Design, Production, and Cost of Satellite Systems," in 28th Annual AIAA/USU Conference on Small Satellites, 2014.

[91] T. Jaeger, W. Mirczak, and B. Crandall, "Cellularized Satellites - A Small Satellite Instantiation that Provides Mission and Space Access Adaptibility," in 2016 Smallsat Conference, Logan, Utah, USA, 2016.

[92] FIPA, "Welcome to the foundation for intelligent physical agents." [Online]. Available: http://www.fipa.org/

[93] H. Zimmermann, "OSI Reference Model-The ISO Model of Architecture for Open Systems Interconnection," IEEE Transactions on Communications, vol. 28, no. 4, pp. 425-432, 1980. 
[94] S. Poslan, "Review of FIPA specifications," 2006. [Online]. Available: http: //www.fipa.org/subgroups/ROFS-SG-docs/ROFS-Doc.pdf

[95] M. Fouquet and M. Sweeting, "UoSAT-12 minisatellite for high performance earth observation at low cost," Acta Astronautica, vol. 41, no. 3, pp. 173-182, 1997.

[96] National Instruments, "Controller Area Network (CAN) Overview (white paper)," Tech. Rep., 2014. [Online]. Available: http://www.ni.com/white-paper/2732/en/

[97] FIPA, "FIPA ACL Message Representation in Bit-Efficient Encoding Specification," 2002. [Online]. Available: http://www.fipa.org/specs/fipa00069/SC00069G.pdf

[98] D. J. Smith, "Understanding terms and jargon," in Reliability, Maintainability and Risk. Elsevier, 2005, p. 17.

[99] H. Pham, "Multi-state k-out-of-n Systems," in Handbook of Reliability Engineering. Springer Science \& Busi- ness Media, 2003, ch. 1.

[100] Real Time Engineers Ltd, "NXP LPC1114 ARM Cortex-M0 Demo." [Online]. Available: http://www.freertos.org/FreeRTOS-for-Cortex-M0-LPC1114-LPCXpresso. html

[101] C. P. Bridges, S. Kenyon, P. Shaw, E. Simons, L. Visagie, T. Theodorou, B. Yeomans, J. Parsons, V. Lappas, and C. Underwood, "A baptism of fire: The Strand-1 nanosatellite," in AIAA/UTU Small Satellite Conference, 2013.

[102] R. Duke, C. Bridges, B. Stewart, B. Taylor, C. Massimiani, J. Forshaw, and G. Aglietti, "Integrated Flight \& Ground Software Framework for Fast Mission Timelines," in 67th International Astronautical Congress, Guadalajara, Mexico, 2016.

[103] K. Laizans, I. Sünter, K. Zalite, H. Kuuste, M. Valgur, K. Tarbe, V. Allik, G. Olentšenko, P. Laes, S. Lätt, and M. Noorma, "Design of the fault tolerant command and data handling subsystem for ESTCube-1," Proceedings of the Estonian Academy of Sciences, vol. 63, no. 2S, pp. 222-231, 2014.

[104] A. O. Erlank and C. P. Bridges, "Satellite Stem Cells - Task allocation demonstration," 2017. [Online]. Available: https://youtu.be/0P54oztI-DI

[105] — "Satellite Stem Cells - State continuity demonstration," 2017. [Online]. Available: https://youtu.be/XgCfr\{_\}s8AOI

[106] A. O. Erlank, "Satellite Stem Cells - Peripheral cross-strapping demonstration," 2017. [Online]. Available: https://youtu.be/-DuyV5RGB5Y

[107] T. Martin, "Chapter 3 - Cortex-M Architecture," in The Designer's Guide to the Cortex-M Processor Family (Second Edition), 2016, pp. 71-130.

[108] N. X. P. Semiconductors, "UM10204 I2C-bus specification and user manual," Semiconductors, vol. 3, no. June, pp. 1-50, 2014.

[109] CubeSpace, "The NEW CubeADCS Y-Momentum bundle is here...," 2016. [Online]. Available: http://www.cubespace.co.za/solutions 
[110] O. R. O. Stohlman, M. Schenk, and V. Lappas, "Development of the Deorbitsail flight model," Spacecraft Structures Conference, no. January, pp. 1-12, 2014.

[111] E. Baceski, S. Gokcebag, A. Erdem, C. G. Erbay, M. Akyol, K. Arslankoz, I. Arslan, M. A. Agca, Y. B. Aydin, A. R. Aslan, and O. Ceylan, "HAVELSAT: A software defined radio experimentation CubeSat," in 2015 7th International Conference on Recent Advances in Space Technologies (RAST). IEEE, jun 2015, pp. 831-834.

[112] J. Gerber, “3-Axis Attitude Control System Hardware Design for a CubeSat," Master's Thesis, University of Stellenbosch, 2014.

[113] P. J. Botma, "The Design and Development of an ADCS OBC for a CubeSat," Master's Thesis, University of Stellenbosch, 2011.

[114] J. Yiu, "What next for microcontrollers?" 2010. [Online]. Available: http://www.embedded.com/-print/4008863

[115] D. Werner, "Smallsat builders admit a little bigger might be a little better," 2017. [Online]. Available: http://spacenews.com/ smallsat-builders-admit-a-little-bigger-might-be-better/

[116] SSTL, “AlSat-1B: Launched 2016,” 2016. [Online]. Available: https://www.sstl.co. uk/Missions/AlSat-1B-Launched-2016

[117] Surrey Satellite Technology Ltd, “OBC750," 2014. [Online]. Available: https: //www.sst-us.com/downloads/datasheets/us \{_\}obdh\{_\}obc750\{_\}2013\{_\}12.pdf

[118] A. O. Erlank, "Parametric CubeSat Database," 2017. [Online]. Available: http://alexerlank.com/wp-content/uploads/2017/09/CubeSat-database-MOD.xlsx 



\section{Appendix A}

\section{Parametric CubeSat Database}

A simplified version of the CubeSat database used in Chapter 2 is presented here. The full database, with all sources listed, is available at [118]. 
7 12/02/2002 MEPSI

8 6/30/2003 AAU CUBESAT 1

9 6/30/2003 CANX-1

10 6/30/2003 CUBESAT XI-IV (CO-57)

11 6/30/2003 CUTE-1(CO-55)

12 6/30/2003 DTUSAT 1

13 6/30/2003 QUAKESAT 1

14 10/27/2005 CUBESAT XI-V (CO-58)

15 10/27/2005 Ncube 2

16 10/27/2005 UWE-1

17 2/21/2006 CUTE 1.7

32 9/22/2006 HITSAT (HO-59)

33 12/16/2006 GENESAT (GeneSat 1)

34 12/20/2006 MARSCOM

35 12/20/2006 MEPSI (MEPSI 2A)

36 12/20/2006 RAFT (NO 60)

37 4/17/2007 AEROCUBE 2

38 4/17/2007 CAPE 1

$394 / 17 / 2007 \quad$ CP3

40 4/17/2007 CP4

41 4/17/2007 CSTB 1

42 4/17/2007 LIBERTAD 1

43 4/17/2007 MAST

44 4/28/2008 AAUSAT 2

45 4/28/2008 CANX 2

46 4/28/2008 COMPASS 1

47 4/28/2008 DELFI C3 (DO-64)

48 4/28/2008 SEEDS 2 (CO-66)

51 11/15/2008 PSSC-Testbed 1

52 1/23/2009 KKS-1 (KISEKI)

53 5/19/2009 AEROCUBE 3

54 5/19/2009 CP 6

55 5/19/2009 HAWKSAT 1

56 5/19/2009 PHARMASAT

57 7/15/2009 BEVO 1

58 7/15/2009 DRAGONSAT 2 (AggieSat 2)

59 9/23/2009 BEESAT

60 9/23/2009 ITu-pSAT 1

61 9/23/2009 SWISSCUBE (SWissCube 1)

62 9/23/2009 UWE-2

63 5/20/2010 HAYATO (K-SAT)

64 5/20/2010 NEGAI-STAR (Negai-Boshi)

65 5/20/2010 WASEDA-SAT2

$66 \quad 07 / 12 / 2010$ STUDSAT

$67 \quad 07 / 12 / 2010$ TISAT 1

68 11/20/2010 O/OREOS

69 11/20/2010 RAX 1 (USA 218)

70 12/08/2010 Mayflower-Caerus

71 12/08/2010 PERSEUS 000

72 12/08/2010 PERSEUS 001

$73 \quad 12 / 08 / 2010$ PERSEUS 002
Size (U) State Architecture Redundancy COTS Subsystems ADCS dev time (months) $\begin{array}{ll}1 \text { DOA } & 0 \text { star } \\ 2 \text { Beacon } & 1 \text { bus }\end{array}$

32 Way Comms 2 multimaster

4 Partial Suc

5 Full Suc

Aerospace Corporation mil

2

University of Aalborg uni

UTIAS (University of Torc uni

University of Tokyo uni

Tokyo Institute of Technc uni

Technical University of $D$ uni

Stanford University uni

University of Tokyo uni

Norweigan Universities uni

University of Würzburg uni

Tokyo Institute of Technc uni

Hokkaido Institute of Tec uni

Stanford University civ

US Naval Academy uni

Aerospace Corporation mil

US Naval Academy

Aerospace Corporation com

University of Louisiana uni

Cal Poly

Cal Poly

Boeing

University of Sergio Arbo uni

Tethers Unlimited. Inc.; F com

University of Aalborg uni

UTIAS (University of Torc un

Fachhochschule Aachen un

Technical University of D uni

Nihon University un

Aerospace Corporation

Tokyo Metropolitan Colle un

Aerospace Corporation mil

Cal Poly

Hawk Institute for Space

Stanford University

University of Texas uni

Texas A\&M University uni

Technical University of B uni

Istanbul Technical Univeı uni

Ecole Polytechnique Fédı uni

University of Würzburg uni

Kagoshima University uni

Soka University uni

Waseda University uni

Indian university consort uni

Scuola universitaria della uni

NASA Ames

University of Michigan un

University of Southern $\mathrm{C}_{i}$ un

Los Alamos National Labı mil

Los Alamos National Labı mil

Los Alamos National Labı mil

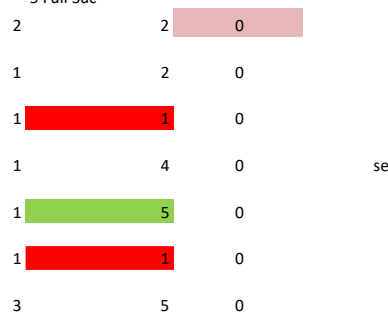

$0 \quad$ none

$\begin{array}{lll}0 & \text { сомм } 24\end{array}$

$0 \quad$ act mag 22

sep beacon $\quad 0 \quad$ pass mag 20

$\begin{array}{lll}0 & 0 & \text { none }\end{array}$

$\begin{array}{lll}0 & \text { act mag } & 29\end{array}$

$\begin{array}{lll}0 & \text { Radio, } \mathrm{OBC} & \text { pass mag }\end{array}$

$\begin{array}{lllllll}1 & 5 & 0 & \text { sep beacon } & 0 & \text { pass mag }\end{array}$

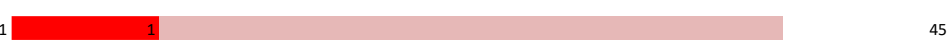

$\begin{array}{lllllll}1 & 3 & 0 & 0 & \text { Radio } & \text { pass mag } & 24\end{array}$

$\begin{array}{llllll}2 & 0 & \text { dual OBC/ func COMMS } & \text { OBC } & \text { act mag }\end{array}$

$\begin{array}{llllll}1 & 4 & 0 & \text { Tx Radio/bat } & \text { Radio } & \text { act mag }\end{array}$

$\begin{array}{llllll}3 & 5 & 0 & \text { Radios } & \text { Radios } & \text { pass mag }\end{array}$

$\begin{array}{llllll}1 & 5 & & & \text { pass mag } & \\ 5 & 0 & 0 & \text { Radio } & 3 \text { axis rw } & 47\end{array}$
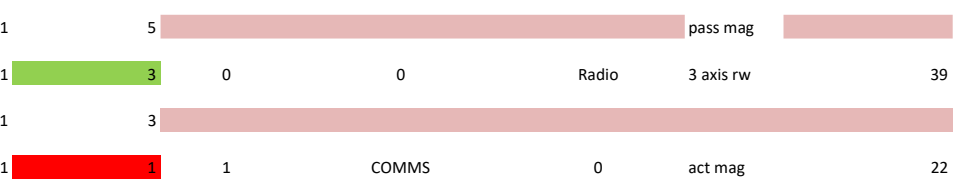

\begin{tabular}{llllcc|c|}
1 & 3 & 1 & Comms & 0 & act mag & \\
1 & 5 & & Radios & Radios & act mag
\end{tabular}

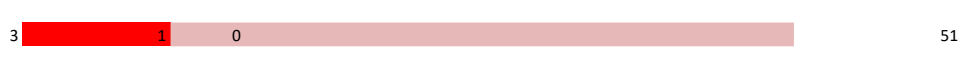

$\begin{array}{llllll}1 & 5 & 2 & 0 & 3 \text { axis } \mathrm{rw}\end{array}$

$\begin{array}{lllll}3 & \text { OBC, func comms } & 0 & \text { act mag } 1 \mathrm{rw} & 40\end{array}$

$\begin{array}{lllllll}1 & 5 & 2 & 0 & 0 & \text { act mag }\end{array}$

$\begin{array}{lllllll}3 & 5 & 0 & 0 & \text { OBC } & \text { pass mag }\end{array}$

\begin{tabular}{l|llll}
1 & 5 & 0 & none & 52
\end{tabular}

$2 \quad 5 \quad$ Radio $\quad$ spin stabil

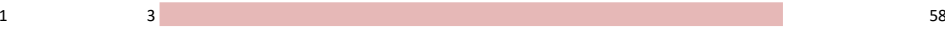

$130 \quad$ heritage radio pass mag $1 \mathrm{n}$ 16
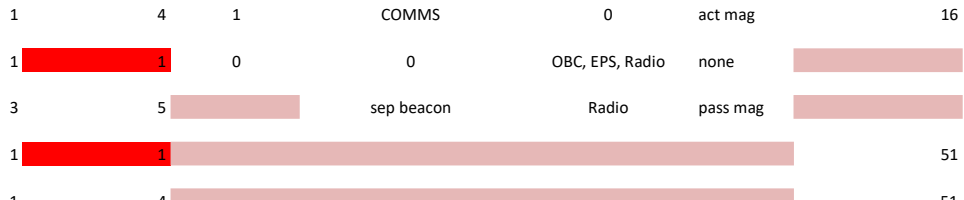

$\begin{array}{llllll}1 & 5 & 2 & \text { OBC, }, \text { COMMS, EPS, ADCS } & \text { COMM } & 3 \text { axis rw }\end{array}$

$\begin{array}{llllll}1 & 2 & 0 & \text { sep beacon } & \text { EPS,OBC,Radio } & \text { pass mag }\end{array}$

$\begin{array}{llllll}1 & 4 & 2 & \text { sep beacon } & 0 & \text { act mag }\end{array}$

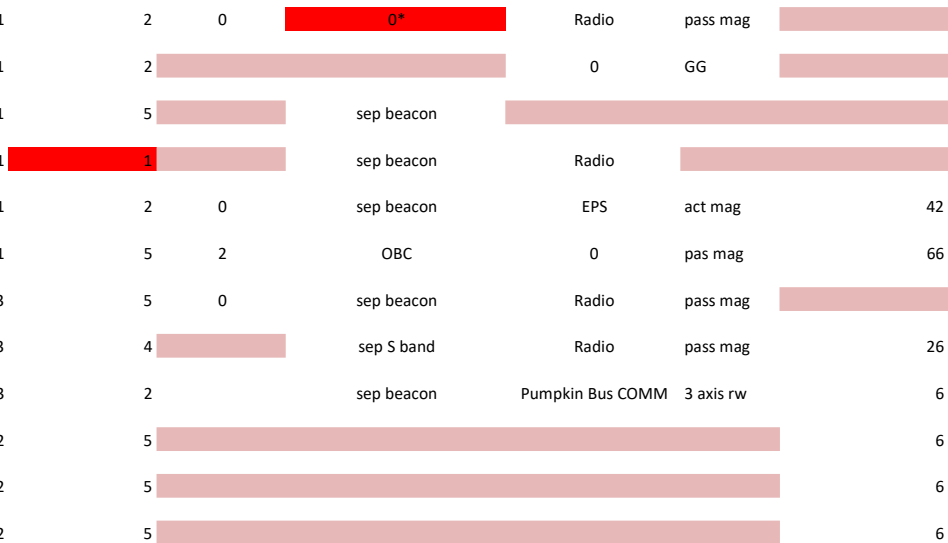




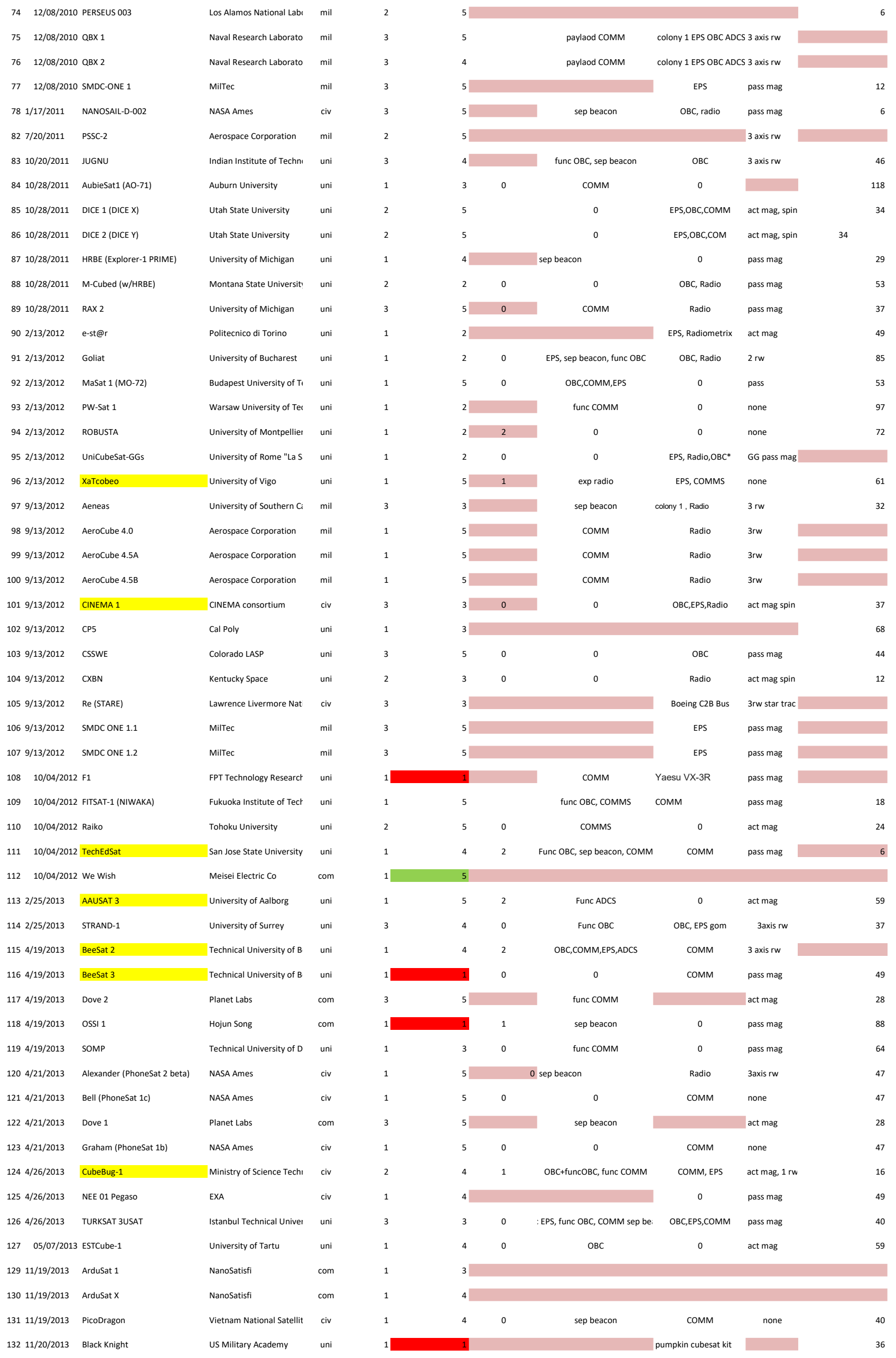




\begin{tabular}{|c|c|c|c|c|c|c|c|c|c|c|}
\hline $13311 / 20 / 2013$ & CAPE 2 & University of Louisiana & uni & 1 & 4 & 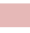 & MCU & . & pass mag & 47 \\
\hline 134 11/20/2013 & ChargerSat & University of Alabama-HI & uni & 1 & 1 & 0 & 0 & 0 & GG & 43 \\
\hline 135 11/20/2013 & COPPER & Saint Louis University & uni & 1 & 1 & 0 & sep beacon & COMM,OBC,EPS & none & 35 \\
\hline 136 11/20/2013 & DragonSat & Drexel University & uni & 1 & 1 & 0 & 0 & EPS, СОMM,OBC & GG & 59 \\
\hline $13711 / 20 / 2013$ & FireFly & NASA Goddard & civ & 3 & 4 & 0 & 0 & $\mathrm{OBC}, \mathrm{COMM}, \mathrm{EPS}$ & act mag, GG & 62 \\
\hline 138 11/20/2013 & Ho'oponopono-2 & University of Hawaii & uni & 3 & 1 & & sep beacon & сомм & GG & 59 \\
\hline 139 11/20/2013 & Horus (STARE-B) & Lawrence Livermore Nat & mil & 3 & 1 & & & Boeing C2B Bus & 3rw star trac & 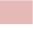 \\
\hline $14011 / 20 / 2013$ & KYSat II & Kentucky Space & uni & 1 & 4 & 0 & 0 & COMM, EPS & pass mag & 35 \\
\hline 141 11/20/2013 & Lunar & Vermont Technical Collę & uni & 1 & 4 & 0 & 0 & EPS,OBC,COMM & pass mag & 47 \\
\hline $14211 / 20 / 2013$ & NPS-SCAT & Naval Postgraduate Scho & uni & 1 & 3 & 0 & sep beacon & COMM,EPS,OBC & none & 71 \\
\hline $14311 / 20 / 2013$ & ORS Tech 1 & Johns Hopkins APL & mil & 3 & 4 & 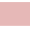 & & & mom biased & 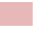 \\
\hline $14411 / 20 / 2013$ & ORS Tech 2 & Johns Hopkins APL & mil & 3 & 4 & 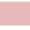 & & 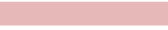 & mom biased & \\
\hline $14511 / 20 / 2013$ & ORSES & MilTec & mil & 3 & 4 & 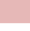 & 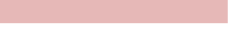 & EPS & pass mag & 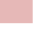 \\
\hline $14611 / 20 / 2013$ & PhoneSat 2 & NASA Ames & civ & 1 & 3 & 0 & sep beacon & сомм & 3 axis rw & 54 \\
\hline 147 11/20/2013 & Prometheus 1.1 & Los Alamos National Labı & mil & 2 & 4 & 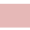 & & colony 2 bus & 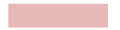 & 26 \\
\hline 148 11/20/2013 & Prometheus 1.2 & Los Alamos National Labı & mil & 2 & 4 & 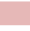 & & colony 2 bus & 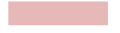 & 26 \\
\hline 149 11/20/2013 & Prometheus 2.1 & Los Alamos National Labr & mil & 2 & 4 & & . & colony 2 bus & 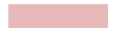 & 26 \\
\hline $15011 / 20 / 2013$ & Prometheus 2.2 & Los Alamos National Labr & mil & 2 & 4 & 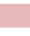 & 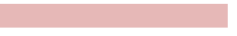 & colony 2 bus & 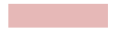 & 26 \\
\hline 151 11/20/2013 & Prometheus 3.1 & Los Alamos National Labc & mil & 2 & 4 & - & 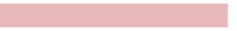 & colony 2 bus & 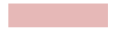 & 26 \\
\hline 152 11/20/2013 & Prometheus 3.2 & Los Alamos National Labı & mil & 2 & 4 & - & 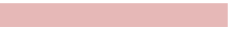 & colony 2 bus & 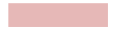 & 26 \\
\hline 153 11/20/2013 & Prometheus 4.1 & Los Alamos National Labr & mil & 2 & 4 & 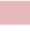 & 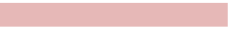 & colony 2 bus & 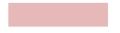 & 26 \\
\hline 154 11/20/2013 & Prometheus 4.2 & Los Alamos National Labr & mil & 2 & 4 & 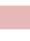 & 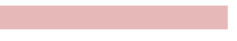 & colony 2 bus & 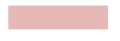 & 26 \\
\hline $15511 / 20 / 2013$ & SENSE SV1 & Boeing & mil & 3 & 4 & ( & ( & colony bus 2, сомM & 3 axis rw & 18 \\
\hline 156 11/20/2013 & SENSE SV2 & Boeing & mil & 3 & 4 & ( & 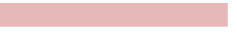 & colony bus 2, сомм & 3 axis rw & 18 \\
\hline 157 11/20/2013 & SPA-1 Trailblazer & University of New Mexic & com & 1 & 1 & 1 & 0 & OВC,EPS, СОMM & pass mag & 12 \\
\hline 158 11/20/2013 & SwampSat & University of Florida & uni & 1 & 1 & 0 & 0 & EPS,COMM & CMGs & 71 \\
\hline 159 11/20/2013 & TechEdSat-3 & San Jose State University & uni & 3 & 4 & 2 & Func $\mathrm{OBC}$, sep beacon, COMM & сомм & pass mag & 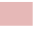 \\
\hline $16011 / 20 / 2013$ & TJ3Sat & Thomas Jefferson High Si & uni & 1 & 1 & 0 & 0 & OBC, СОMM & none & 83 \\
\hline $16111 / 21 / 2013$ & CINEMA 2 (KHUSat-1) & KyungHeeUniversity & $\mathrm{civ}$ & 3 & 3 & 0 & 0 & OBC,EPS, Radio & act mag spin & 52 \\
\hline $16211 / 21 / 2013$ & CINEMA 3 (KHUSat-2) & KyungHeeUniversity & civ & 3 & 3 & 0 & 0 & OBC,EPS, Radio & act mag spin & 52 \\
\hline $16311 / 21 / 2013$ & CubeBug 2 (Manolito) & Ministry of Science Techı & civ & 2 & 4 & 0 & OBC+funcOBC, func COMM & COMM, EPS & act mag, 1 rw & 23 \\
\hline $16411 / 21 / 2013$ & Delfi-n3Xt & Technical University of D & uni & 3 & 5 & 1 & func comm, $\mathrm{OBC}$ & сомм & 3 axis rw & 73 \\
\hline $16511 / 21 / 2013$ & Dove 4 & Planet Labs & com & 3 & 4 & & & & & 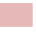 \\
\hline $16611 / 21 / 2013$ & First-MOVE & Technical University of $\mathrm{N}$ & uni & 1 & 3 & 0 & 0 & COMM,EPS & pass mag & 95 \\
\hline $16711 / 21 / 2013$ & FUNcube 1 & Amsat-UK & civ & 1 & 4 & 0 & 0 & EPS gom & pass mag & 59 \\
\hline $16811 / 21 / 2013$ & GATOSS (GOMX 1) & GOMSpace & com & 2 & 4 & 1 & 0 & EPS,OBC,COMM & act mag & 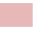 \\
\hline 169 11/21/2013 & HiNCube & Narvik University College & uni & 1 & 1 & 1 & 0 & EPS, СОMM,OBC & act mag & 95 \\
\hline $17011 / 21 / 2013$ & HumSat-D & University of Vigo & uni & 1 & 4 & & 0 & EPS & none & 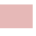 \\
\hline 171 11/21/2013 & ICube 1 & Institute of Space Technc & uni & 1 & 2 & 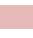 & 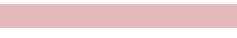 & & pass mag & 59 \\
\hline $17211 / 21 / 2013$ & NEE 02 Krysaor & EXA & civ & 1 & 4 & 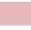 & 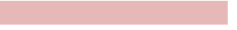 & 0 & pass mag & 56 \\
\hline $17311 / 21 / 2013$ & OPTOS & INTA & $\mathrm{civ}$ & 3 & 3 & 2 & 0 & EPS & mom biased & 71 \\
\hline $17411 / 21 / 2013$ & PUCP-SAT 1 & Pontifical Catholic Univeı & uni & 1 & 3 & ( & & & pass mag +2 & 48 \\
\hline $17511 / 21 / 2013$ & Triton 1 & ISIS-BV & com & 3 & 4 & & & COMM, EPS,OBC & & 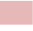 \\
\hline $17611 / 21 / 2013$ & UWE 3 & University of Würzburg & uni & 1 & 4 & 0 & OBC, СОМM & сомм & mom biased & 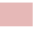 \\
\hline $17711 / 21 / 2013$ & VELOX-P 2 & Nanyang Technological L & uni & 1 & 3 & 0 & 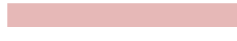 & & act mag & 59 \\
\hline 178 11/21/2013 & ZACUBE 1 & Cape Peninsula Universit & uni & 1 & 4 & & 0 & OBC,EPS & act mag & 49 \\
\hline $179 \quad 12 / 06 / 2013$ & 3 Aero-Cube 5a & Aerospace Corporation & mil & 1 & 4 & 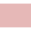 & сомм & Radio & $3 \mathrm{rw}$ & 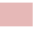 \\
\hline $180 \quad 12 / 06 / 2013$ & 3 Aero-Cube $5 b$ & Aerospace Corporation & mil & 1 & 4 & ( & сомм & Radio & $3 \mathrm{rw}$ & . \\
\hline $181 \quad 12 / 06 / 2013$ & 3 ALICE & Air Force Institute of Tec & mil & 3 & 4 & 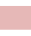 & & Colony 1 Bus & act mag & 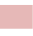 \\
\hline $182 \quad 12 / 06 / 2013$ & CUNYSat-1 & City University of New Yc & uni & 1 & 1 & & sep beacon & & & 59 \\
\hline $183 \quad 12 / 06 / 2013$ & 3 FIREBIRD 1 & Montana State Universit! & uni & 2 & 4 & 0 & 0 & OBC, EPS,COMM & pass mag & 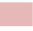 \\
\hline $184 \quad 12 / 06 / 2013$ & 3 FIREBIRD 2 & Montana State Universit & uni & 2 & 4 & 0 & 0 & OBC, EPS,COMM & pass mag & ( \\
\hline $185 \quad 12 / 06 / 2013$ & 3 IPEX & Cal Poly & civ & 3 & 4 & & & & pass mag & 23 \\
\hline $186 \quad 12 / 06 / 2013$ & M-Cubed-2 & University of Michigan & uni & 1 & 4 & 0 & 0 & сомм & pass mag & 78 \\
\hline
\end{tabular}


187 12/06/2013 SMDC-ONE 3.1 missing 2.1 and 2 . MilTec

188 12/06/2013 SMDC-ONE 3.2

$189 \quad 12 / 06 / 2013$ SNAP 1

190 12/06/2013 TacSat-
MilTec

Naval Postgraduate Scho

AFRL mil
4 



\section{Appendix B}

\section{Artificial Cell Schematics}

The full schematics of the testbench artificial cell, described in Chapter 7, are given here. 